Florida International University

FIU Digital Commons

FIU Electronic Theses and Dissertations

University Graduate School

7-16-1997

\title{
The role of individualism and collectivism as predictors of attributions for unethical work behavior : an empirical examination across two culturally diverse groups
}

Ana Azevedo

Florida International University

DOI: $10.25148 /$ etd.FI14032385

Follow this and additional works at: https://digitalcommons.fiu.edu/etd

\section{Recommended Citation}

Azevedo, Ana, "The role of individualism and collectivism as predictors of attributions for unethical work behavior : an empirical examination across two culturally diverse groups" (1997). FIU Electronic Theses and Dissertations. 1353.

https://digitalcommons.fiu.edu/etd/1353

This work is brought to you for free and open access by the University Graduate School at FIU Digital Commons. It has been accepted for inclusion in FIU Electronic Theses and Dissertations by an authorized administrator of FIU Digital Commons. For more information, please contact dcc@fiu.edu. 


\section{FLORIDA INTERNATIONAL UNIVERSITY}

Miami, Florida

THE ROLE OF INDIVIDUALISM AND COLLECTIVISM AS

PREDICTORS OF ATTRIBUTIONS FOR UNETHICAL WORK BEHAVIOR:

AN EMPIRICAL EXAMINATION ACROSS

TWO CULTURALLY DIVERSE GROUPS

A dissertation submitted in partial satisfaction of the

requirements for the degree of

DOCTOR OF PHILOSOPHY

IN

BUSINESS

by

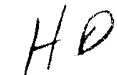

27.99

A $9 \%$

Ana Azevedo

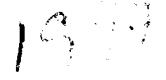

(1.) 
To: Dean Harold E. Wyman

College of Business Administration

This dissertation, written by Ana Lucia Boamorte de Azevedo, and entitled The Role of Individualism and Collectivism as Predictors of Attributions for Unethical Work Behavior: An Empirical Examination Across Two Culturally Diverse Groups, having been approved in respect to style and intellectual content, is referred to you for judgement.

We have read this dissertation and recommend that it be approved

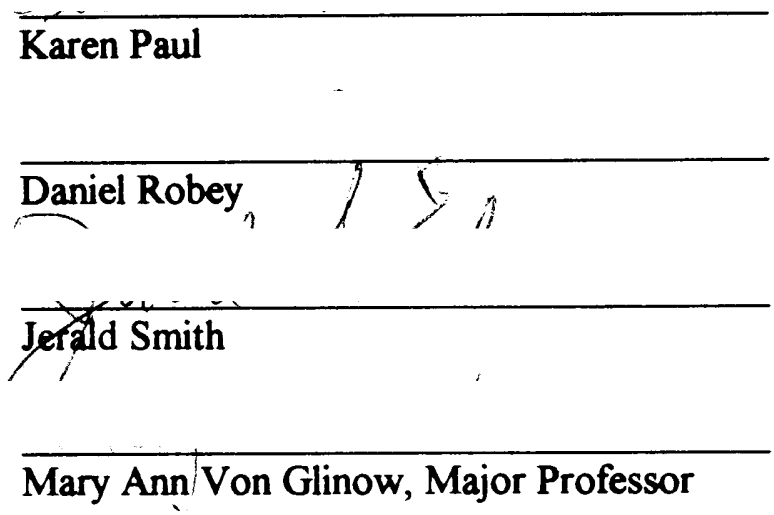

Date of Defense: July 16,1997

The dissertation of Ana Lucia Boamorte de Azevedo is approved.

Dean Harold E. Wyman

College of Business Administration

Dr. Richard L. Campbell

Dean of Graduate Studies

Florida International University, 1997 
I dedicate this dissertation to my family: mom and dad, Carmen, Claudia, Luis

Daniel, Gustavinho, Gaia, Kali, Pequeno, Nuvem and Bobby. Without their encouragement, patience and most of all love, the completion of this work would not have been possible. 


\section{ACKNOWLEDGMENTS}

I wish to thank the members of my committee for their comments and wonderful support. I also want to thank Dr. Michael Mullen, for his expertise, and for performing the crucial role of statistical advisor. My dear colleagues Lori Zalka and Ellen Drost, significantly contributed to different parts of this manuscript. I am in debt to so many professors at FIU and FAU, for allowing me to distribute questionnaires to their students. Without their consideration, this work would never have gotten done. Ruth Chapman helped me put this document into decent shape. She, together with few other secretaries at FIU, has served me tremendously, from beginning to end. Barbara Garrett was also wonderful, keeping me registered in spite of all the problems.

A special thanks must go to Dana Farrow and Daniel Robey, for their support throughout the entire program, and for believing in me in crucial moments. I will also always be grateful to Mary Ann Von Glinow, for recognizing my value to a greater extent than I did, and for renewing my confidence in such a way that it kept me going. 
ABSTRACT OF THE DISSERTATION

THE ROLE OF INDIVIDUALISM AND COLLECTIVISM AS

PREDICTORS OF ATTRIBUTIONS FOR UNETHICAL WORK BEHAVIOR:

AN EMPIRICAL EXAMINATION ACROSS

TWO CULTURALLY DIVERSE GROUPS

by

Ana Azevedo

Florida International University, 1997

Professor Mary Ann Von Glinow, Major Professor

This dissertation addresses how the cultural dimensions of individualism and collectivism affect the attributions people make for unethical behavior at work. The moderating effect of ethnicity is also examined by considering two culturally diverse groups: Hispanics and Anglos. The sample for this study is a group of business graduate students from two universities in the Southeast. A 20-minute survey was distributed to master's degree students at their classroom and later on returned to the researcher. Individualism and collectivism were operationalized as by a set of attitude items, while unethical work behavior was introduced in the form of hypothetical descriptions or scenarios. Data analysis employed multiple group confirmatory factor analysis for both independent and dependent variables, and subsequently 
multiple group LISREL models, in order to test predictions. Results confirmed the expected link between cultural variables and attribution responses, although the role of independent variables shifted, due to the moderating effect of ethnicity, and to the nuances of each particular situation. 


\section{TABLE OF CONTENTS}

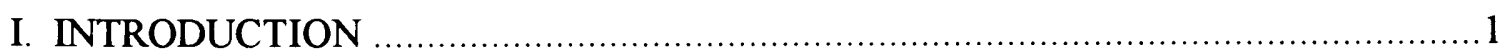

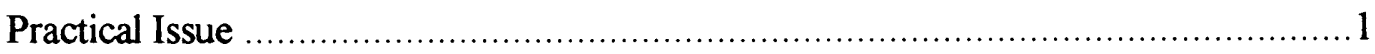

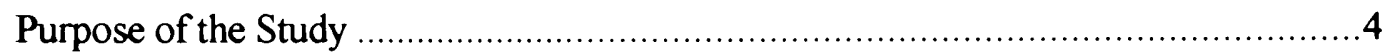

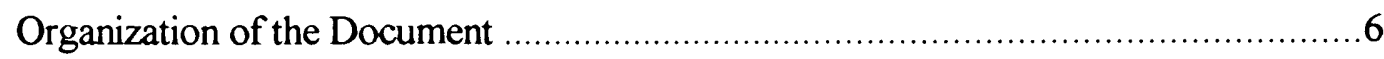

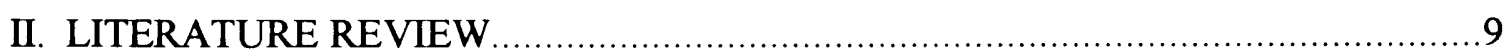

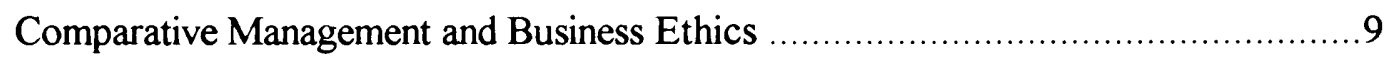

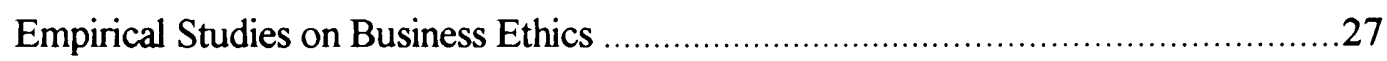

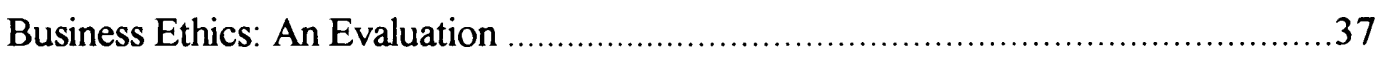

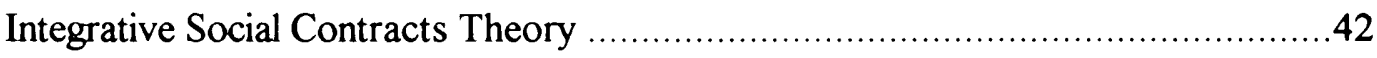

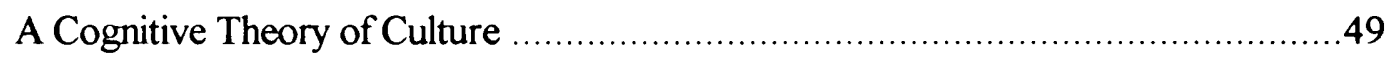

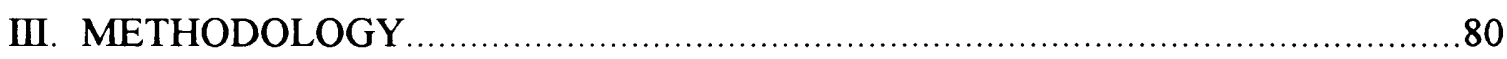

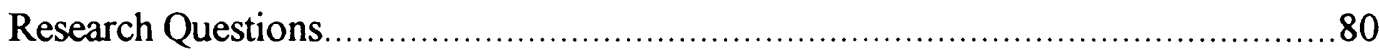

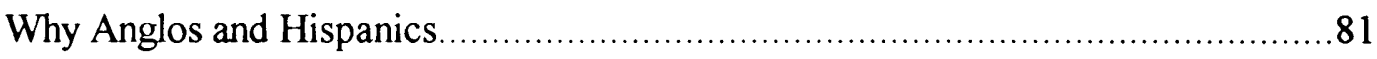

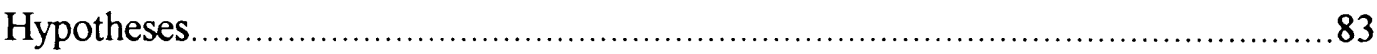

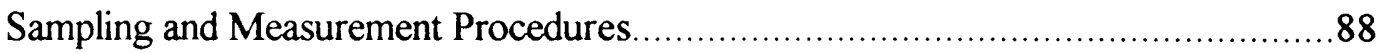

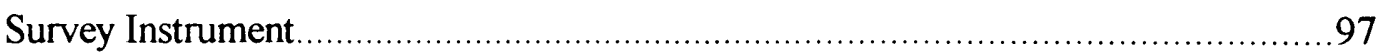

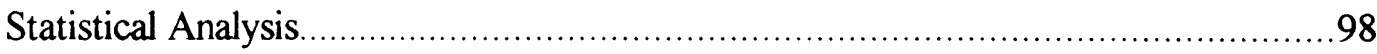


V. RESULTS

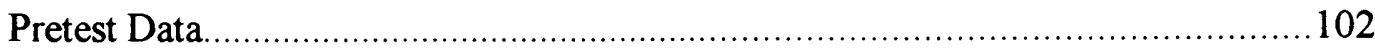

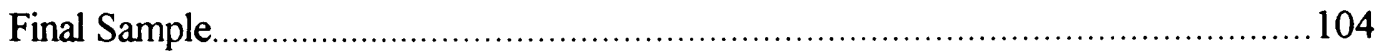

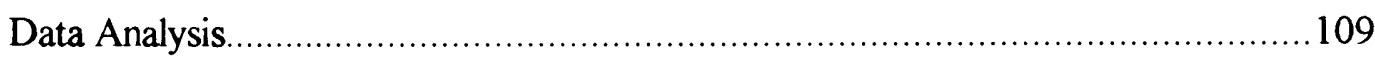

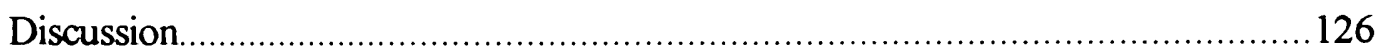

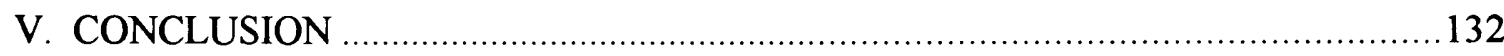

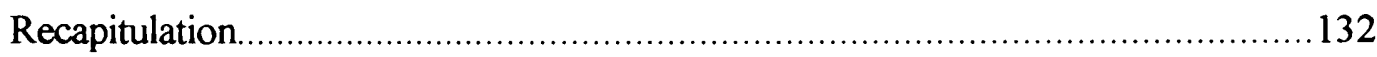

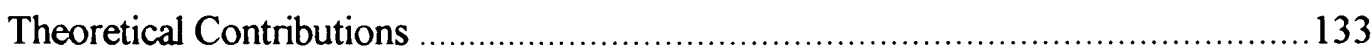

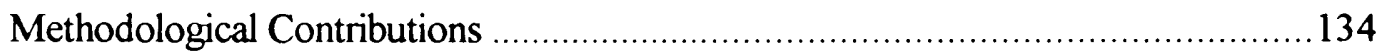

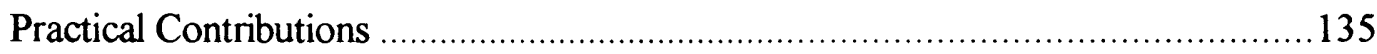

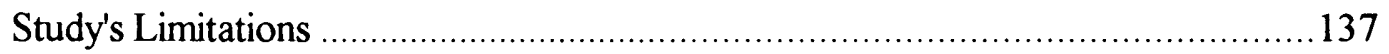

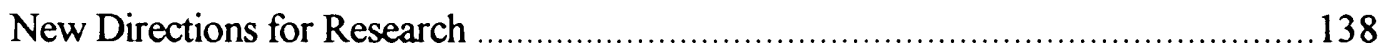

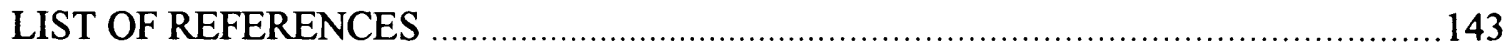

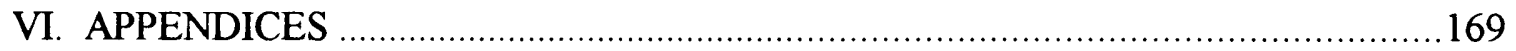

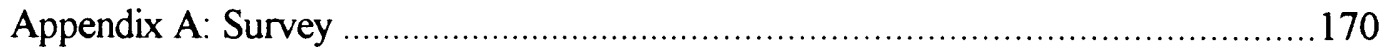

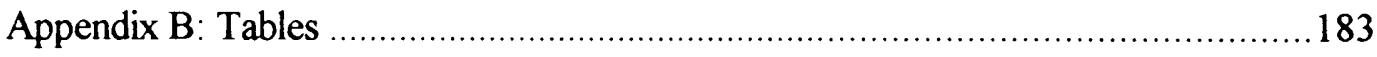




\section{CHAPTER 1}

\section{INTRODUCTION}

"Managing the global enterprise and modern business management are becoming synonymous. The term international can no longer be relegated to a subset of organizations or to a division within the organization...In fact the very concept of domestic business may have become anachronistic." (Adler, 1991: 3-4)

\section{Practical Issue}

The globalization of business is a central trend in modern business activity. International trade, global strategic alliances and worldwide operations are becoming increasingly important. Multinational corporations are the leaders in this movement. Since World War II, they quickly spread in different frontiers, building substantial corporate size and power. Their evolution however, is filled with ethical controversies in such delicate issues as bribery, environmental pollution, unfair competition and so on (Donaldson, 1989).

While corporate behavior within national boundaries is regulated somewhat efficiently by domestic laws, international activities in host countries are often subject to ambiguous interpretation due to disparities in cultural norms, which leave multinational behavior less 
susceptible to societal control. Managers in local, yet culturally diverse, companies may also find themselves with fewer clear guidelines to assist decision-making. Unfortunately, awareness of the importance of cultural values for effective management is offset by the lack of knowledge concerning cross-cultural effects on ethical reasoning and judgment, which makes moral assessments in decision-making hard to understand.

Therefore, among the biggest challenges in the development of a global economy is the need to work towards international ethical understanding. The increasing globalization of companies requires them to consciously create a common ground that "...emerge(s) from the degree of agreement among societies, corporations and other organizations regarding the appropriate ethical frameworks and behaviors in a given situation" (Buller, Kohls,\& Anderson, 1991:768). Without establishing a global ethic that accommodates cultural differences, there can be no basis for settling international conflicts among different entities.

An international ethical framework can aid businesses in globalization by providing a basis for settling agreements on company practices with respect to cultural commonalities, while also allowing differences to be safeguarded. Companies can agree to search for a core set of ethical principles, but they must also leave some room for corporate behavior that reflects cultural differences (Okleshen \& Hoyt, 1996). In any case, it is the companies' consent to the establishment and enforcement of ethical principles that can bring stability to international business activity, which still awaits better definitions concerning required 
behavioral norms and the boundaries of "admissible behavior" (Vernon-Wortzel, 1994; Buller, Kohls, \& Anderson, 1991).

Cross-cultural studies can serve an important role in this process of globalizing ethics: by explaining how cultural values affect an individual's definitions of ethics, they help delineate both a 'moral free space' (not sanctioned by the international community) for accommodating culture-specific norms, and the foundations for the development of universally-held (generally accepted and enforced) 'hypernorms' (Donaldson \& Dunfee, 1995).

One way of addressing these similarities and differences is to examine how cultural values affect the rationale an individual gives for obeying or transgressing social norms. Culture may provide overriding principles for behaving ethically or unethically (Carroll \& Gannon, 1997). The knowledge of such principles can be a starting point for the generation of more widely accepted ethical codes, even within cultural communities (Schlegelmilch \& Houston, 1989).

In fact, the implementation and enforcement of corporate ethics codes involves a similar dilemma: unless individuals' cultural bases for obeying or transgressing organizational norms are well understood, such codes are likely to remain largely inefficient. This is not only 
relevant for multinational corporations; today even 'domestic' companies need sensitivity to deal with a culturally diverse workforce (Robey, 1993).

In this study I examine how organizational members with different ethnic backgrounds justify unethical actions. The knowledge of such behavioral attributions might contribute to better design, implementation and enforcement of corporate ethical codes since it allows organizations to align social norms with their community's needs (Wood, 1994). In collectivist countries, for example, ethical principles may be built around more personalized, informal arrangements that guarantee considerable protection for employees. In individualist countries other issues may be more important, such as how to preserve some degree of individual freedom or how to harmonize individual needs with society's larger demands. In each case, an understanding of the effect of cultural influences on cognitive processes should be a starting point for a more effective control of behavior in the workplace (Trevino \& Nelson, 1995).

\section{Purpose of the Study}

This study investigates individuals' attributions for unethical behavior across two different cultural communities (see below). Cultural variables (individualism and collectivism) are expected to account for different rationalizations concerning social norm transgression. 
Unethical behavior is operationalized through decision-outcomes of business-related scenarios that depict situations involving bribery, conflict of interest, environmental pollution, and paternalism. These scenarios were previously developed according to business ethics literature (Fritzsche \& Becker, 1983), and were further tested and refined through successive empirical investigations. Decision-outcomes, however, were either adapted or created for the purpose of this study. Also, a set of attribution statements for each scenario was originally introduced. These statements provide a list of possible causes or reasons for the alleged unethical decisions.

Integrative Social Contracts Theory (ISCT) (Donaldson \& Dunfee, 1994) is used as the theoretical foundation for this cross-cultural examination. This theory provides the conceptual basis for understanding ethics as a community-based social contract. The theory also addresses the need to specify two sets of social norms: a microsocial contract, which incorporates community-specific values and practices; and a macrosocial contract, based on general, widely accepted ethical principles. The framework therefore seems well suited to address the dilemmas posed by the multicultural businesses.

The Anglo (White, non-Hispanic American) and Hispanic (American or Latin American) communities were chosen for comparison, on the basis of their expected opposition in scores of individualism and collectivism. The selection of business graduate 
students from two universities in the Southeast allows the final sample composition to be somewhat balanced between these two ethnic groups.

\section{Organization of the Document}

Since this study addresses a comparative management problem, Chapter 2 begins by acknowledging the research that attests to the influence of culture on management processes. These studies provide strong evidence of the lack of universalism in management thinking and practice and of the need to investigate how cultural values affect cognitive and behavioral responses.

Before examining cross-cultural studies that specifically address differences in ethical perceptions, attitudes and behavior across communities, it is useful to introduce a historical perspective of the field of business ethics, in order to call attention to its rapid evolution and to the increasing relevance of its central issues. This presentation is organized around the main theoretical perspectives that provide an anchor to the field, followed by a discussion of the remaining deficiencies in dealing with current ethical dilemmas faced by organizations.

Also, to justify the theoretical framework here employed, a review of the empirical research is undertaken to show what kinds of ethical issues have been addressed by the literature. As I conclude, the existing empirical models are inadequate in answering questions 
relevant to this study. However, the main contributions of cross-cultural studies on ethics are summarized, as well as some of the important individual, organizational, and situational factors highlighted by other empirical work on the field. These factors constitute potential moderator variables that may need to be controlled within the study design.

The theory of ethics chosen for this study is then introduced and evaluated in terms of its strengths and weaknesses in addressing my research objective. ISCT's novelty is weighted against its promising aims. I finalize the chapter by discussing Triandis' theory of culture, wherein individualism and collectivism are seen as 'underlying themes' that organize a set of interrelated ideas. Together, these two theoretical frameworks (of ethics and culture) provide the conceptualizations needed to examine the link between cultural variables and the types of justifications people give for unethical work behavior.

Chapter 3 introduces the main research questions of this study and the appropriate methodology for their investigation. The foundations for examining the Anglo and Hispanic groups are discussed in detail. Two hypotheses address 1) the link between the independent variables - individualist and collectivist attitudes - and the dependent variables, attributions for unethical work behavior and 2) the role of ethnicity as a moderator of these relationships. Sampling and measurement procedures are specified, together with instrument selection criteria. The statistical analysis section describes model testing and other issues of construct validation. 
Chapter 4 presents final results from the statistical analysis. After pretest and final sample description, multiple group confirmatory factor analysis and LISREL models are introduced. Findings are evaluated in terms whether they support the study's hypotheses.

Chapter 4 also provides a more detailed discussion of the statistical results. After elaborating the research findings for each business ethics scenario independently, an attempt is made to integrate them by examining how the role of each cultural variable changes across situations, as well as across the two ethnic groups.

Chapter 5 offers some concluding thoughts, which address the study's main contributions, as well as its limitations. New directions for theory building and practice are also discussed. 


\section{CHAPTER 2}

\section{LITERATURE REVIEW}

"The most spectacular illustrations of the extent to which normality may be culturally defined are those cultures where an abnormality of our culture is the cornerstone of their social structure...A recent study of an island of northwest Melanesia by Fortune describes a society built upon traits which we regard as beyond the border of paranoia. In this tribe the exogamic groups look upon each other as prime manipulators of black magic, so that one marries always into an enemy group which remains for life one's deadly and unappeasable foes. They look upon a good garden crop as a confession of theft, for everyone is engaged in making magic to induce into his garden the productiveness of his neighbors'; therefore no secrecy in the island is so rigidly insisted upon as the secrecy of a man's harvesting of yams. Their polite phrase at the acceptance of a gift is, "And if you now poison me, how shall I repay you this present?" " ( Ruth Benedict, 1934, as in Pojman, 1989:21).

\section{Comparative Management and Business Ethics}

Comparative management concepts can be applied to investigate how cross-cultural differences affect individuals' attributions for unethical behavior. Comparative studies reveal 
the importance of culture in explaining cognitive and behavioral processes; they offer a detailed conceptualization of culture and a framework for conducting cross-cultural examinations.

Comparative Management: What do we know?

The field of comparative management has evolved somewhat slowly since the early 1960 s with the systematic cross-cultural investigation of similarities and differences among managerial actors, processes, structures and functions, in order to provide better prescriptions concerning managerial effectiveness in international contexts (Boddewyn, 1969). Among the reasons for studying comparative management, are the interdependency of global economic institutions and the consequent need to develop higher cultural sensitivity. The study of other cultures is also useful in expanding individuals' knowledge base beyond their common, preconceived ways of understanding the world (Nath, 1986).

McClelland's (1961) work on motivational needs provided one of the first important contributions to the field. The author examined the achievement motive (nAch) in 1,300 children's stories across 40 different countries and found that culture had a significant effect on the rate of economic growth. 
Although the concept of culture plays a central role in the comparative management literature, the topic received very little attention for a number of years. Difficulties in operationalizing the concept may explain the lack of interest on the part of researchers working in the field. Smircich (1983) has suggested, for example, that there are at least five distinct conceptualizations of culture being used in organizational analysis - culture is viewed as: an instrument serving human needs (from functionalism), or an adaptive-regulatory mechanism (from structural-functionalism), or a system of shared cognitions (from ethnoscience), or a system of shared symbols and meanings (from symbolic anthropology), or a projection of men's universal unconscious mind (from structuralism).

Another possible explanation for the scarcity of comparative studies, is that organizational researchers still suffer from parochialism - trapped in their own views and selfcontained problems, they are often unable to truly engage in cross-cultural research (Boyacigiller \& Adler, 1991). This is unfortunate, considering the unsustainable expectations concerning the universal applicability of management principles (cf. Hofstede, 1993; Negandhi, 1973, 1974, 1975).

Some researchers believe that the international corporate evolution will bring even higher demands for cultural sensitivity. While today multinational companies may survive in an environment of high product standardization and low-cost strategy, the global enterprises of tomorrow will face different challenges, with mass-customization and multiple-efficient 
strategies posing the need for better cultural understanding related to specific market niches (Adler \& Ghadar, 1990).

Among the most popular frameworks for building knowledge of cross-cultural differences are Kluckhohn and Strodtbeck's value orientations (1961) and Hofstede's dimensions of culture (1980a, 1980b, 1983, 1984). I shall address the main contributions of these authors, after providing a classical definition of culture:

"Culture consists of patterns, explicit and implicit of and for behavior acquired and transmitted by symbols, constituting the distinctive achievement of human groups, including their embodiment in artifacts; the essential core of culture consists of traditional (i.e., historically derived and selected) ideas and especially their attached values; culture systems may, on the one hand, be considered as products of action, on the other, as conditioning elements of future action." (Kroeber \& Kluckhohn, 1952)

This definition stresses the learning and sharing aspects of cultural transmission, as well as the circular, and mutually reinforcing, links between culture and behavior. Culture evolves through the internalization of behavioral habits or patterns; shared meanings grow independent from particular situations, thus becoming objectified (Berger \& Luckmann, 
1966). As the programs of the mind, cultural meanings provide strong guidance for future action (Hofstede, 1980b).

As shown in Table 1, the relationship between culture and behavior can be depicted in a cycle that initially evolves with behavioral patterns producing cultural meanings. These meanings then, through time, become internalized and objectified; as "objective reality", they further shape personal values, attitudes and, ultimately, behavior. Once a cultural-behavioral link is established and becomes ingrained within a society, this cycle is self-reinforcing, thus leading to cultural persistence.

In this cyclical mechanism, attributions for behavioral acts may be viewed not only as a precondition for the creation of cultural meanings, but also as byproducts of them. In other words, individuals from a particular culture draw upon these crystallized meanings to justify their behavioral actions. In this sense, culture works like a broad, foundational 'schema' (Gray, Bougon, \& Donnelon, 1985).

In an early study of a Jazz Orchestra, Bougon, Weick, and Binkhorst (1977) showed, for example, that members of this organization shared similar causal maps for interpreting work events. Further organization studies following this cognitive orientation have not only been able to replicate the idea of causal maps, but also to apply different techniques for 
uncovering people's mental programming, such as adaptations of Kelly's Repertory Grid, or the use of scripts and communication rules (cf. Smircich, 1983).

The work of Harry Triandis $(1972,1989,1990,1993,1994)$ - adopted as the theoretical basis for this study's cross-cultural investigation- also builds on a cognitive view of culture, wherein the elements described in Table 1 (behavioral patterns, attributions, values etc) are seen as contents of a particular schema or cognitive structure.

Aside from an understanding of the concept of culture, the study of cross-cultural differences requires a framework that specifies the relevant dimensions for comparison. So, for example, Kluckhohn \& Strodtbeck's (1961) six value orientations provide such basis by contrasting societies in terms of their views concerning individual nature (good, good and evil, or evil), relationships with the world (dominance, harmony, or subjugation), with other people (individualist or collectivist), with respect to their primary type of activity (doing, controlling, or being), and according to their orientation in time (past, present, or future) and space (private, public, or mixed).

Similarly, Hofstede's cultural dimensions (1980a, 1980b) allow cross-cultural comparison based on the following dimensions: individualism-collectivism (the extent to which people define themselves as individuals or members of a group), power distance (the degree of acceptance, by the less powerful individuals, of an unequal distribution of power), 
uncertainty avoidance (the extent to which society members feel threatened and try to avoid ambiguous situations) and masculinity-femininity (the extent to which society's dominant values emphasize assertiveness and materialism or relationships with people and quality of life). The author arrived at these dimensions by factor-analyzing questionnaire responses of more than a hundred thousand employees that worked in an American multinational company (IBM) across forty different countries (Hofstede, 1980a). This study was later expanded to include fifty three countries; the findings revealed that national culture accounted for the differences in work-related values and attitudes of multinational employees matched according to position within the organization, profession, age and gender (Hofstede, 1983).

Hofstede's work also provided a preliminary mapping of a few country clusters, which inspired future research into the understanding of such cultural similarities (cf. Ronen \& Shenkar, 1985). The author further extended his investigations into the study of organizational cultures, showing that while national cultures were highly correlated with work values, the former seemed to better account for differences in perceived organizational practices (Hofstede et al., 1991). Although not free of criticism, Hofstede's cultural dimensions (three out of the four) were shown to align closely with an Eastern-developed Chinese Value Survey; as a consequence of this work a fifth dimension emerged - "Confucian dynamism" - capturing the contrast between a long-term and short-term orientation in life (Hofstede \& Bond, 1988; Bond \& Mai, 1989). 
Consistent with the comparative management literature, cross-cultural investigations in psychology also found cultural influences in basic psychological processes such as learning and reinforcement, social perception and attribution (see below). Out of the five Hofstede's dimensions, psychologists devoted their attention to the individualism-collectivism distinction, since it better captured the relationship between self and others (Hofstede, 1994). In a review of this work, Kagitcibasi and Berry (1989) listed a series of cognitive-behavioral correlates of individualism-collectivism, demonstrating the amount of excitement of researchers with the usefulness of these concepts. Attribution theory studies for example, found no "fundamental attribution error" (the tendency to invoke personal dispositions in the explanation of other people's behavior) in the collectivist South India (Miller, 1984); likewise, Kashima and Triandis (1986) showed that the Japanese demonstrated a much less "self-serving bias" when compared to the Americans.

Other important findings from this stream of work addressed cultural differences in issues of distributive justice, reward allocation and strategies for conflict resolution. The greater emphasis on ingroup harmony by collectivists was evidenced in a preference for conflict avoidance techniques and in the choice of equality and need (versus equity) as the norms for resource allocation (cf. Berman, Murphy-Berman,\& Singh, 1985; Berman, Murphy-Berman, Singh,\& Kumar, 1984; Leung 1985, 1987; Triandis, Leung, Villareal,\& Clack, 1985; Yang, 1981; Marin, 1985; Hui, 1984). 
To conclude, the growth of comparative management research brought significant contributions to organization studies, beyond the simple assertion that management theories do not apply abroad. Better conceptualizations and operationalizations of culture are now available, and study designs have become more attentive to issues such as the distinction between country and national differences and the need to control for contextual and environmental variables, such as technology, size, political, legal and economic conditions (Ricks, Toyne, \& Martinez, 1990).

Further organization theory development requires boundary specification, greater exposure of researchers to different cultural contexts, and multicultural teams in the processes of theory development and testing (Doktor, Tung \& Von Glinow, 1991).

\section{Business Ethics: A Historical Overview}

The understanding of unethical behavior required by this study is sought from the growing literature on business ethics. Although the field is relatively young, it has provided important contributions to the specification of contextual, organizational and individual influences on (un)ethical responses (Trevino \& Nelson, 1995). Cross-cultural studies on business ethics also have begun to accumulate evidence concerning the effects of national culture on individuals' ethical perceptions, attitudes, and judgements (Carroll \& Gannon, 
1997). As an introduction to the subject, the following paragraphs describe the evolution of business ethics as a field of inquiry.

There is a growing interest in the topic of business ethics. The daily incidence of unethical behavior on the part of organizational members provides clear evidence of the need to foster higher ethical standards within the business community (Tsalikis \& Fritzsche, 1989). Catastrophes such as the 1984 Union Carbide disaster at India and NASA's involvement in both the 1986 Challenger explosion and the 1987 Hubble Space Telescope fiasco serve as critical reminders of the dangerous implications of unethical decisions (Velasquez, 1988; Whyte, 1989; Steiner \& Kanter, 1993; Quinn \& Walsh, 1994). Considering that "...the list of corporate crises and disasters is endless and growing" (Shrivastava \& Mitroff, 1987:6), including plant accidents, severe environmental pollution, product injuries, sabotage, and terrorism, top management strategies must become open to specific mechanisms that induce higher ethical sensitivity in employees at all organizational levels.

Responses to this challenge are coming in many different ways. Worldwide, corporations are taking steps towards the institutionalization of ethics through the implementation and enforcement of written codes of conduct (Mathews, 1987; Schlegelmilch \& Houston, 1989). Although the effectiveness of such codes has been questioned, their impact is bound to be enhanced by the use of additional mechanisms such as training 
programs, ethics committees and judiciary boards (cf. Delaney \& Sockell, 1992; Weber, 1981).

On the academic side, business schools have altered their curricula to address business/government/society relationships. At the root of this movement, a series of Harvard Business Review articles during the 1950s highlighted society's greater awareness of social responsibility issues (Boulding, 1952; Ohmann, 1955 \& 1957; Demos, 1955; Campbell, 1957; Johnson, 1957; Broehl, 1958). By the end of the decade and under the sponsorship of institutions such as Ford and Carnegie Foundation, the inclusion of integrative courses linking organizations to their broader socio-economic-political environment began to spread across the nation (Paul, 1987). It was the beginning of the development of business ethics as a field.

Since then, academic interest on the topic has grown in many different research avenues. Capitalizing on the diverse background of researchers, studies quickly proliferated in a wide spectrum of topics, covering both macro issues - e.g. Capitalism and the Economy; Metaethics; Management Practices of Specific Industries - and narrower, business-specific topics - e.g. Corporations and their Functional Areas; Managers and Employees, and the Multinational Corporations or MNCs (Fleming, 1987). Other central issues included corporate social responsibility and social performance (Davis, 1973; Carroll, 1979; Cochran \& Wood, 1984; Boal \& Perry, 1985; Wood, 1991), business ethics in a global environment (Sethi, 1977 \& 1986; MacMillan, 1978; Evans, 1981; Vogel, 1982; Fritzsche \& Becker, 1983 
\& 1984), public policy and strategic management (Fleming, 1981; Fahey \& Narayanan, 1986; Preston, 1986; Epstein, 1987) and, the teaching of business ethics (Conrad, 1978; Buchholz, 1979; Preston, 1984; Hoffman \& Moore, 1982).

With just over thirty years of empirical research and after slow growth during the 1960s, the field of business ethics is now progressing at a faster pace. With the Academy of Management's creation of the Social Issues in Management Division in 1971, the American Association of Collegiate Schools of Business' 1980 request for inclusion of "ethical considerations" in MBA programs (followed by the development of business and society courses at the undergraduate level) and further benefiting from the emergence of few important publication outlets (e.g. Business \& Society, Journal of Business Ethics, Business Ethics Quarterly), there is a good amount of excitement concerning research opportunities for scholars working in the field (Paul \& Matasar, 1993).

\section{Business Ethics: Main Theoretical Perspectives}

Ethics is an "inquiry into the nature and grounds of morality"; it addresses "...what is good and evil, what is right and wrong, and thus is an inquiry into what we ought and ought not to do" (Beauchamp \& Bowie, 1983; DeGeorge, 1983). It is a practical, systematic application of moral values and codes of conduct to the resolution of complex problems 
(Churchill, 1982). By offering standards to guide behavioral actions, ethics serves as an important normative foundation for individuals, organizations, and societies.

Moral standards are perceived as different than other societal standards in that they address issues with serious consequences to the welfare of human beings (e.g. murder, rape, theft, fraud, child abuse). In this respect, they also seem to elicit stronger emotions and move people beyond their self-interest and towards impartiality. Although often confused as legal matters, moral standards cannot be subjected to judgments made by specific authoritative bodies (Velasquez, 1988).

Considering the challenges that business organizations face today, in terms of corporate misconduct, an understanding of morality within the context of daily work activities can offer a promising aim: that of transforming how organizations operate. Thus the study of ethics was brought to the environment of big corporations.

The field of business ethics attempts to apply society's set of moral rights and wrongs to the particular reality of organizations. It views business policies, rules, institutions, and behavior through the lens of morality. Both corporations as a whole and specific individuals working for them are held morally responsible for their ways of conduct. Implicit in this central argument is the acknowledgment of the power of organizations to impact their social environment (Certo, 1992). 
Business ethics research, therefore, evolves through moral or ethical reasoning, "...the reasoning process by which human behaviors, institutions, or policies are judged to be in accordance with or in violation of moral standards" (Velasquez, 1988:25). This process is conducted by applying ethical theory principles. In order to understand how this critical analysis is actually performed, I review its supporting frameworks.

As summarized in Table 2, the main theoretical approaches to moral reasoning can be categorized into three groups: teleological (e.g. utilitarianism; egoism), deontological ( e.g. Kant's categorical imperative; the Golden Rule) and relativism. Theories of rights and justice usually represent a combination of both teleological and deontological principles (cf. Reidenbach \& Rubin, 1990; Tsalikis \& Fritzsche, 1989).

The first group of theories - the teleological - is also known as 'consequential', since evaluations concerning the morality of any particular action are based on the analysis of its consequences. In the case of utilitarianism -although with slight variations according to the specific theory (e.g. act utilitarianism or rule utilitarianism)- the righteousness of an action depends on how much good or evil it brings for those involved. The best act is the one that optimizes this ratio. For egoism, only the consequences to the particular individual are considered relevant input to this calculus. 
Deontological theories, on the other hand, are 'non-consequential' - they rely solely on ethical principles to evaluate the morality of an action. So in Kant's categorical imperative the ethical rule is : "I ought never to act except in such a way that I can also will that my maxim should become a universal law" (Kant, 1959/1964). Although often ignored, Kant's categorical imperative resembles Nietzche's idea of "eternal return": "...to live in a manner that we would want to live still once more and that we would want to live this way forever" (cf. Marton, 1992/1993). In both there is an ethical obligation to submit each individual action to a general norm. The popular principle "Do unto others as you would have them do unto you" ( the 'Golden Rule') constitutes another example of this philosophical tradition.

Teleological and deontological theories have been criticized on different grounds. The former approach is often accused of ignoring "blatant wrongs", of conflicting with basic justice principles, and of being unequipped to deal with serious problems in business practices (e.g. discrimination, pollution). The latter approach, although preferred by contemporary philosophers, provides rules that are considered imprecise, unlimited, and insensitive to the necessary situational contingencies (Tsalikis \& Fritzsche, 1989).

Some theories have attempted to overcome these criticisms by using both consequential and nonconsequential strengths. Theories of rights, for example, extend upon Kant's categorical imperative by specifying, from a moral standpoint, the activities or interests individuals are authorized or empowered to pursue (Velasquez, 1988); individuals' autonomy 
is therefore established within certain boundaries. On the other hand, moral duties are also acknowledged as individuals rights' counterparts. In Locke's (1690) classical Two Treatises, natural rights are associated with the preservation of life, equality and liberty.

Theories of justice are rooted in ethical principles such as equality, freedom, and fairness (e.g. Aristotle, 1877; Nozick, 1974; Rawls, 1971). Individuals' actions are said to be just provided that they guarantee equal treatment or equal allocation of society's goods (egalitarianism); or result from free choice (libertarianism); or rely on an agreed fair method for settling social conflicts.

Egalitarian claims, although appealing, are often hard to sustain; can individual differences be ignored? In fact, the Aristotelian fundamental "principle of formal justice" ("equals ought to be treated equally, and unequals ought to be treated unequally" (cf. Reidenbach \& Rubin, 1990:650) was further elaborated by philosophers to provide different bases for distributive justice: individual's contribution (effort, ability), needs or rights, and societal contributions. Interestingly, one of the distinguishing features of capitalist economies as compared to socialism is the former's conception of justice based on the individual's contribution while the latter seems to rely more heavily on the individual's needs.

Libertarians view free individual choice as the key factor in determining justice . By postulating the supremacy of 'freedom from coercion', libertarians offer little room for 
accommodating other rights or values. Although different types of freedom may be acknowledged (freedom from hunger or ignorance), societies have no basis, for example, to protect the disadvantaged. A more challenging critique to the libertarian logic is that it provides no account for the fact that one's individual freedom constrains another's (Velasquez, $1982 / 1988)$.

Finally, in the most comprehensive theory of justice, Rawls (1971) proposed two basic principles for settling social conflicts in a fair manner: the equal liberty principle and the difference principle. In the former, equal rights and basic liberties of individuals are secured but in compatibility with the liberties of others; in the latter, social and economic inequalities allow exceptions in applying rules uniformly.

In addition to this set of justice principles, Rawls also suggested what he considered a fair method for evaluating the adequacy of any moral principles - the 'original position': a group of rational self-interested people would choose the governing principles for living in a future, hypothetical society without any previous knowledge of what specific characteristics (e.g. sex, race, abilities, social and economic position, etc) they would eventually display (this method is referred as contractualism and has its roots on Locke, Rousseau and Kant). Many criticisms were directed at the 'original position', as well as at the two justice principles. However, Rawls's theory stands as one of the most detailed accounts of morality. 
The last group of theories -ethical relativism- refutes the idea of universally held ethical principles or rules. Moral beliefs are said to vary with the situation, or more generally, with the particular culture under consideration (Benedict, 1934; Harman, 1975). Fundamental differences among the value systems of individuals, groups, and societies are taken as evidence of the impossibility of embracing universal ethical norms. Although relativism addresses the promising aim of cultural tolerance, it is often subject of criticism for offering the opposite extreme of universalism: can't any common moral concerns be found across populations/environments? Some have argued that relativism implies the absence of any grounds for objective morality assessment:

"Moral concepts, insofar as they possess objectivity, gain legitimacy only through the habits and attitudes of a given culture; in turn, all intercultural comparisons of values are meaningless" (Donaldson, 1989)

In spite of its strong criticism, the above argument exposes a highly acknowledged problem with relativism, i.e., its unsustainable logic. As this paper shall explain in greater detail, Integrative Social Contracts Theory (Donaldson \& Dunfee, 1994) offers a middle ground solution to this issue by showing how relativistic claims can be accepted within certain boundaries which safeguard the adherence of societies to a broader set of more fundamental ethical principles. However, before introducing this theoretical framework, I provide a description and evaluation of the empirical work conducted by business ethics researchers. 


\section{Empirical Studies on Business Ethics}

There are few streams of research dealing with the prediction of (un)ethical behavior within organizations. They offer fairly encompassing models which attempt to delineate the conditions or factors that affect ethical judgments and behavioral intentions.

There are also a number of cross-cultural investigations which generally assess the influence of national culture on ethical perceptions, beliefs, attitudes and behavioral intentions. Although various instruments have been developed to measure these dependent variables, little attention has been given to the measurement of culture.

\section{Ethical Decision-Making Models}

Among the most influential models of ethical decision-making are Hunt and Vitell's (1986/1992), Ferrell and Gresham's (1985) and Trevino's (1986). I will address the main theoretical components of each model, making reference to the supporting empirical evidence, whenever available.

In Hunt and Vitell's (1986/1992) 'General Theory of Marketing Ethics', the authors outlined a model in which the deontological and teleological traditions are combined to explain how individuals actually make ethical judgments. To be sure, both the inherent 
righteousness of actions and behavioral consequences are considered by organizational members in choosing a specific course of action as the most ethical alternative. Ethical judgements, together with teleological evaluations (also considered independently of the former) are hypothesized to affect individual actions through their impact on 'intentions to behave'. The model depicts therefore a cognitive-behavioral link that is initiated with the perception of an ethical problem and subsequent consideration of alternatives for its resolution. The background variables reflect the influences of cultural, industrial and organizational norms, as well as of individuals' personal experiences on this cognitive process.

Hunt and Vitell's (1986/1992) model has been tested in a series of marketing studies which overall provided general support for its basic propositions (Mayo \& Marks, 1990; Singhapakdi \& Vitell, 1990, 1991; Hunt \& Vasquez-Parraga, 1993; Vitell, Rallapalli, \& Singhapakdi, 1993; Singhapakdi \& Vitell 1993). The authors have revised their model on the basis of these findings, providing more detailed specification for the role of personal and environmental variables. Research on the model, however, is still incipient and both theoretical (e.g., the role of organizational cultures; the relative importance of deontological and teleological evaluations) and measurement issues (related to the operationalization of its core variables) need further refinement. So far, none of the empirical studies has addressed how different national cultures influence ethical perceptions, cognitions and behaviors, although Vitell, Nwachukwu and Barnes (1993) have advanced some testing propositions. 
Ferrel and Gresham' s (1985) 'Contingency Framework for Understanding Ethical Decision-Making' also postulates the influence of social-cultural variables and of individual factors (e.g. values and attitudes) on individual decision-making, but expands on the role of situational components such as 'significant others' and 'opportunity'. The authors build on differential association and role-set theory to explain how (un)ethical behavior is affected by close personal relationships or role-set configuration. In addition to the existence of professional codes (the basic organizational culture factor in the former model), the framework also incorporates organizations' reward systems as a key variable in limiting or stimulating particular behaviors of individuals in their work setting (Jansen \& Von Glinow, 1985). Although supported by previous marketing studies (cf. Zey-Ferrell, Weaver \& Ferrell 1979; Zey-Ferrell \& Ferrell, 1982), this model has not been directly tested.

In Trevino's (1986) 'Person-Situation Interactionist Model' individual and situational factors are examined in a rather interactive fashion. Central to individuals' cognitive responses to an ethical dilemma is their stage of cognitive moral development, a crucial component in the major theoretical accounts of moral reasoning (Kohlberg, 1976, 1981, 1984; Rest, 1979, 1986). The situational components of the model resemble closely Ferrel and Gresham's (1985), but different individual variables are considered: locus of control, ego strength and field independence. 
Based on a slight variation of this model, Trevino and Youngblood (1990) designed an experiment to assess individuals' influence (the "bad apples" argument) and situational effects (the "bad barrels" argument) on ethical decision-making behavior. This latter component of the model was operationalized with vicarious learning manipulations of reward and punishment, which were hypothesized to affect the dependent variable through the mediating process of outcome expectancy, consistently with the postulates of social learning theory. The individual factors of cognitive moral development and locus of control were measure with Rest's (1971) DIT and Rotter's (1966) instruments, respectively. Findings supported the expected relationships advanced by the model (for individual and situational factors), with the exception of the direct effects of vicarious learning on (un)ethical behavior.

The similarities among these ethical decision-making models has already been acknowledged. Ferrell, Gresham and Fraedrich (1989) for example, compared Hunt and Vitell's framework with Ferrell and Gresham's and suggested a synthesis for existing ethical decision-making models. Although Trevino's model is not explicitly addressed, the central role of individual's level of cognitive moral development in responses to ethical issues is recognized.

In reviewing four causal models in business ethics research (Hunt \& Vitell's, Ferrell \& Gresham's, Trevino's, and the 1987 Bommer, Cratto \& Gravander's), Brady and Hatch (1992) raised serious doubts concerning their contribution to the field - What is gained 
through the use of ethical decision-making models? According to the authors, although the proponents of these models claim their status as theories, this claim seems largely unwarranted, since they offer only general propositions which "solve no problems" (1992:309). They are also imprecise in addressing the role of individual and situational moderators, which are aggregated indiscriminately in order to (allegedly) increase predictive power. Therefore, the models can only serve as a weak defense of the need to validate moral truths with empirical evidence.

Therefore, although aimed to discuss "what is going on inside organizations", ethical decision-making models fail to bridge this reality with the ideal moral standards that constitute the basic motivation for the study of ethics. By emphasizing the descriptive approach, ethical decision-making models fall short:

"Kantians, for example, would argue that ethical behavior is not a function of moderating factors at all. Indeed, the whole point of developing an ethic is to free oneself from environmental influence and to make right choices and to be satisfied with the actions these choices direct, no matter what the world offers up as enticement or reward. Ethics, they say, is about freedom from external pressure and about overcoming one's personal limitations rather than giving in to them." (Brady \& Hatch, 1992:313) 
In this respect, the empirical tradition in business ethics research, by preventing itself from the use of any normative frameworks, offers no reasonable set of principles that can help businesses to conform to higher ethical standards. Instead, largely subscribing to a situational view of ethics, this tradition can only provide a mapping of the particular conditions that affect (un)ethical behavior.

For the purpose of this study, however, it is important to highlight the most relevant empirical findings that relate these situational, organizational and individual factors to the outcomes of ethical judgment/decision-making, behavioral intentions and acceptance of ethical norms. These factors constitute potential moderator influences on the cultureattribution relationship.

As shown in Table 3, situational variables such as the type of ethical problem or the specificity of the particular context were found to affect ethical decision-making. Samples of both managers and students demonstrated different sensitivity to varying ethical issues; also, work activities (especially in marketing) seemed to exert a great degree of pressure which compromised the ethicality of individual decisions. 
Relevant organizational factors included tenure, position, and most notably organizational culture. Individuals who stayed longer in the organization or worked in top management positions (as opposed to middle management) displayed higher ethical standards in their judgements. The culture of the organization was also found to have an important influence on ethical decision-making; organizational members relied heavily on the company's normative structure and especially on referent others (e.g. peers, immediate superior) for guidance concerning the appropriate ways of conduct. Although the evidence concerning the effectiveness of ethical codes and policies is controversial, an adequate control system of rewards and punishments may prove helpful in enhancing ethical standards, aside from the more recent mechanisms for institutionalizing ethics (e.g. ethics training programs). Finally, in spite of few attempts to show that bigger organizations exhibit more ethical problems, conclusions from research have been largely unsupportive (cf. Longnecker, McKinney, \& Moore, 1989).

The most impressive findings relate to individual factors. As shown in Table 3, several studies have shown a strong positive relationship between age and ethical judgments, i.e., older subjects demonstrated much higher sensitivity to ethical issues. The level of cognitive moral development (CMD) was also a significant factor in five different studies. Higher stage individuals were more ethical in their decisions; in addition, other findings suggest that CMD is associated with cheating, resistance to authority pressure and helping behavior (cf. Trevino \& Youngblood, 1991). 
Few studies reported a positive influence of personal characteristics such as internal locus of control (apparently allowing greater resistance from external pressure), and individual values (e.g., being responsible and honest). On the other hand, Machiavelism had a negative impact on ethical judgments, reflected in its overreliance on self-interested actions (cf. Singhapakdi \& Vitell, 1990). The findings about gender were the most controversial: although there were a good number of studies showing women to make more ethical decisions, the contrasting evidence (no gender differences) was equally compelling (see Table 3).

To conclude, it is noteworthy that in these research streams there has been not a single investigation of behavioral attributions. Since the bulk of this empirical work relies strongly on an attitude-driven framework (beliefs and attitudes affect intentions to behave, which in turn affect actual behavior), the role of behavioral attributions is ignored.

Cross-Cultural Studies on Business Ethics

The second important group of empirical studies examines ethical perceptions, attitudes and judgments across culturally diverse samples, in search of differences that might be attributed to their distinguishing cultural background. Findings provided by this line of research offer the closest empirical indication of the relationship between national culture and ethics. 
The present author has found only fourteen studies that address this relationship. Seven of them confirmed expected variations in (perceived) ethical sensitivity to particular dilemmas, typically introduced to subjects through the manipulation of scenarios. Researchers also investigated individuals' ratings of ethicality associated with a set of critical ethical issues highlighted by the business ethics literature, such as employee misconduct (e.g., bribery, violation of company's policies, misuse of company's assets), misuse of proprietary or sensitive information, or the carrying of improper political activities (cf. Robertson \& Schlegelmilch, 1993; Mitchell, Lewis \& Reinsch, 1992).

Among the samples studied, a large variety of countries were found: United States, United Kingdom, China, Japan, Korea, Singapore, Taiwan, Greece, France, Germany, Denmark, New Zealand, Australia, Nigeria and Israel. Only one study (Tsalikis \& Nwachukwu, 1988) compared two cultural communities - Blacks and Whites - within the same country (United States), and reported similar ethical beliefs across them.

The remaining seven cross-cultural studies provided contradictory evidence on the effects of national culture: they concluded that ethical perceptions were invariant across a series of different nationalities (see Table 4). Unfortunately, these findings taken together suggest significant confusion and controversy as to whether the study of national culture may contribute to a better understanding of ethical perceptions, judgments and ultimately, behavior. 
One might attribute this controversy to the lack of appropriate research designs. Since the independent variable, culture, is never actually measured, how can we know what specific aspects of culture may explain the hypothesized differences? So far, the effects of culture remain largely assumed. Secondly, there may be also a good degree of confounding with other variables, possibly operating at different levels of analysis (e.g., the legal and political environment, organizational and individual factors), since these research designs have not attempted to control for alternative explanations. Therefore, it is hard to sustain that the alleged differences or similarities on ethical perceptions, attitudes and judgments were due to relevant or irrelevant cross-cultural effects.

It is interesting to notice that none of the studies developed specific hypotheses linking dimensions of culture with particular ethical responses. Without an understanding of how these dimensions can explain such responses, there is no clear guidance on what samples to choose or on how to operationalize the variables. Although a good amount of effort has been placed on the development of instruments that adequately map a variety of (un)ethical situations, the ways in which culture may or may not affect these situations should be examined a priori. 
In fact, it is possible that culture may be an important concept only for certain types of ethical responses, and that there may be significant similarities across cultures operating simultaneously. The theoretical framework used by this study adopts this integrative view.

Future studies on cross-cultural business ethics need to pay greater attention to those methodological issues if they want to contribute to a better delineation of when (and how) national culture affects ethical perceptions, attitudes and judgments:

"Thus, the relevant question to ask is when does culture influence (ethical reasoning) rather than if it does or does not" (Adler, 1988/1992: preface, viii)

\section{Business Ethics: An Evaluation}

After reviewing the philosophical and empirical frameworks employed by business ethics researchers, it is important to evaluate them in relation to this study's objective. Business ethics as a field provides no theoretical framework which can provide a conceptual basis for this study. While the philosophical tradition provides highly abstract models, the empirical work in business ethics is mostly descriptive. This is an unfortunate outcome of communication failure between two research communities, which not only impedes the field's progress, but also limits the relevance of the existing theoretical contributions. Integrative 
Social Contracts Theory is the only suitable model to examine cross-cultural effects on individuals' attributions for unethical behavior.

The Gap between the Normative and Empirical Approaches

This paper has highlighted the criticisms made of theoretical perspectives on business ethics in an attempt to show that there is no comprehensive theory that will allow individuals to arrive at proper moral judgments.

Another question is the level of analysis issue: Can immoral acts be blamed on organizations as a whole, or should it ultimately be traced back to the particular individuals involved in the situation? The implications to organizations and to society in general of proper accountability cannot be overemphasized.

Peculiar to the field, however, is the potential for individual/collectivity conflict, a problem that Aram (1989) referred to as the 'paradox of interdependent relations' : "...the desirability of freedom for one individual or organization (is confronted) against the requirements for security and stability of a larger collectivity" (1989:267). Although some authors have attempted to emphasize possible congruence of interests (a rationale similar to Adam Smith and the "invisible hand"), until business practitioners can rely on a sensible set of solutions that adequately addresses this problem, knowledge about ethics will remain 
largely academic, with no practical contributions for business management. Without adequate prescriptions, it is unlikely that practitioners will minimize their resistance to the increasing demands for higher ethical sensitivity on their part.

The underlying issue in this argument is, therefore, the legitimacy of business ethics as a field. While business researchers can often be criticized for their managerial biases and for neglecting the socio-political implications of business activities, reverting this to the opposite extreme may seem unwarranted (and politically dangerous). Steidlmeier (1992) for example, calls for an abandonment of the 'atmosphere of confrontation' - society's attack on businessmen for their "misbehavior" - in favor of a defense of ethics as a positive quest for human happiness and fulfillment.

A related challenge to the field's legitimation is the need for integration between two streams of research: the purely normative, prescriptive approach, mostly the product of philosophical inquiry, and the empirical, positivist orientation that characterizes business ethics researchers. In this respect, the normative nature of the philosophical approaches reviewed earlier provides intellectual inspiration to business ethics researchers evaluating organizational actions ex post, but offers no general framework for testing specific hypotheses concerning actual behavior of organizational members. 
The lack of collaboration from the part of the philosophical and business research communities has two striking implications for the issue of legitimacy: first, moral theories are unequipped to provide specific guidelines to address the ethical problems faced by the business community - their level of abstraction is basically too high. Second, empirical findings that expose the current ethical dilemmas encountered by organizational members are discarded as useless for the purpose of validating philosophical claims concerning human nature and behavioral consequences (Greenberg \& Bies, 1992). Although ethical decision models were developed to address this deficiency, they failed to bridge their prescriptions with a normative conception of ethics.

The absence of integration between the "is and ought to" also prevents the use of more traditional methodologies aimed at theory testing. While normative research may apply 'critical thinking' to a particular business situation, the empirical work has been mostly descriptive (Fleming, 1987). The field is thus accused of being both theoretically and methodologically "soft" (Paul \& Matasar, 1993).

After interviewing thirty-two researchers working in the field, Kahn (1990) suggested the use of guiding images to help build an agenda for business ethics research:

"Taken together, the four images (ethics as conversation, history, vision and community) suggest that an ideal ethical system is one in which members 
strive to make a set of connections: with one another, in dialogue; with the historical antecedents of present patterns of thought and action; with the ideals they clarify and imagine; and with the larger community of diverse members straining toward shared goals" (Kahn, 1990:)

To conclude, although the interdisciplinary nature of the field of business ethics could be considered a strength, it has worked instead as a development constraint, given the inability of the philosophical and business research communities to share their world views.

As a consequence of this gap, the existing theoretical models fall short of delivering an adequate integration between ethical principles and behavioral prescriptions. Ethics becomes, therefore, either an ideal unreachable system (too abstract) or a set of situational components too broad to be meaningful (solely context-dependent).

Donaldson and Dunfee's 'Integrative Social Contracts Theory'(Donaldson \& Dunfee, 1994) (ISCT) is a framework that attempts to bridge the normative and empirical perspectives. In fact, these authors take as a starting point the need to consider both commonly-held ethical principles (the basis for converging on a set of widely-shared ethical principles) and the cultural/contextual foundation that limits the universality and applicability of any ethical framework. 
As noted before, the importance of this integration for the development of international business ethics understanding is invaluable; it is also crucial to the effectiveness of corporate ethical codes. ISCT seems therefore an adequate conceptual foundation for the present study.

\section{Integrative Social Contracts theory}

Donaldson and Dunfee's (1994) ISCT attempts to address the interconnections between the normative and empirical research streams through a contractarian approach that incorporates two kinds of social contract: a 'macro' theoretical contract which specifies general, normative rules and a 'micro' implicit contract, verified within the boundaries of specific communities, thus reflecting their cultural patterns and shared agreements.

The authors build on the assumptions of 'moral rationality' and 'moral free space'. The former is said to be 'bounded' in three specific ways: by a finite capacity of moral agents to process ethically relevant information; by the limitations of ethical theories in revealing moral truths; and by the "plastic or artifactual nature of economic systems and practices" (Donaldson \& Dunfee, 1994:258). Taken together, these characteristics of 'boundedness' imply not only that there is significant amount of moral uncertainty, but also that ethical norms must be circumscribed within the confines of communities' practices. 
ISCT integrates these community-specific practices through the terms of a microsocial contract: that is, community members are given a 'moral free space' to delineate a set of ethical norms which address their particular business practices (e.g. gift-giving, corporate paternalism, nonmonetary compensation). Also, general agreement among different communities is sought through a macrosocial contract, which incorporates broad, general principles (called 'hypernorms') such as the preservation of life and liberty, and environmental protection. These broader principles establish boundaries or limits to the terms of the microsocial contract.

The theory applies social contract thinking (or contractualism) to arrive at these two contracts; this reasoning is a traditional hypothetical method in normative philosophy, which is intended to assure fairness in the process of contract specification. Rational agents would therefore - through the method of contractualism, here based on a consensus strategy - agree on a set of general principles or 'hypernorms' (the 'macro social contract') to determine morality in economic transactions. They would also safeguard enough freedom ('moral free space') to accommodate their economic, cultural, political and religious concerns in the form of specific, community-level 'micro contracts'. Aside from this, the terms of the macro social contract should also preserve individuals' right to exit any particular community. 
The first two principles of the macro social contract are therefore:

"1. Local economic communities may specify ethical norms for their members through microsocial contracts; (and)

2. Norm-specifying microsocial contracts must be grounded in informed consent buttressed by a right of exit." (Donaldson \& Dunfee, 1994:

Yet, informed consent and right of exit can only speak to the authenticity of norms. They offer a rationale for a communitarian approach that secures specific ethical norms compatible with communities' shared understandings. But, as the authors acknowledge, the theory would provide only a version of cultural relativism if no limits were accepted for contractors' moral free space.

Hence, in order to achieve legitimacy, community-level ethical norms have to be consistent with basic fundamental principles or 'hypernorms'. Although few sources may be used as a basis for determining these principles (e.g. Universal Declaration of Human Rights), interestingly, it is the criterion of convergence among communities' beliefs which is offered as the key (convergence of ethical norms doesn't define 'hypernorms' per se, but rather informs the search process). Finally, priority rules should also be developed to settle conflicts among communities' legitimate rules. 
The remaining two principles of the macro social contract specify that:

"3. In order to be obligatory, a microsocial contract norm must be compatible with hypernorms; (and)

4. In case of conflicts among norms satisfying Principles 1-3, priority must be established through the application of rules consistent with the spirit and letter of the macrosocial contract". (Donaldson \& Dunfee, 1994:265, 269).

ISCT may be criticized for its overreliance on freedom of choice. In less developed countries, as the theory's proponents acknowledge, it may be particularly difficult to talk about 'informed consent' or 'right to exit'. More importantly, as I shall elaborate upon, communities' reasons for abiding or transgressing a social contract can be expected to vary across cultures. While in some countries a strong value may be placed on individual choice, others rely more heavily on obedience or duty (Hofstede, 1980a; Triandis, 1993). The language of ISCT, as well as its contractarian approach, denote an individualistic worldview (cf. Nelson, 1994). However, as Walzer (1992) admitted, moral language is never unexpressive or culturally neutral.

In spite of the simplicity imposed by a hypothetical method, the theory offers two major contributions to business ethics research. First, as mentioned earlier, it provides a good 
rationale for integrating the seemingly disparate normative and empirical perspectives, a request made by several authors working in the field. To be sure, community-specific norms reveal shared understandings that guide actual behavior in organizations; they detail what has been called a lower-order or situational ethics (Williams \& Coughlin, 1993). Hypernorms, on the other hand, constitute a higher, more basic foundation for how individuals in specific communities ought to behave, and therefore provide the basis for delineating a normative dimension.

Secondly, ISCT reconciles contrasting empirical findings concerning similarities and differences between communities, a problem acknowledged in cross-cultural studies as the convergence-divergence controversy (cf. Ralston, Gustafson, Cheung, \& Terpstra, 1993). The concept of national culture is often used to explain differences between communities or societies while their similarities are attributed to the spread of industrialization. It is hardly ignored that these processes of convergence and divergence may in fact coexist, with different cultures achieving similar attitudes or values through, for example, the effects of multinational efforts in marketing/advertising, while within countries differentiation of cultural values and practices is maintained and further reinforced through mechanisms of market segmentation. Cross-cultural researchers, however tend to consider these processes as mutually exclusive. Robey and Azevedo (1994) argued that this same type of myopia also occurs in studies of organizational persistence and change. A theoretical framework that allows the simultaneous consideration of community-based norms, reflective of distinctive value systems, and a 
broader, more widely accepted set of norms, which captures similarities, offers, therefore, a promising avenue for future work.

Through this integration, ISCT addresses deficiencies in the business ethics literature associated with the abstract nature of the existing philosophical approaches and with the often limited situational view of empirical models. The theory is also well suited to the practical issue of this study: international frameworks and corporate ethical codes need to embrace both generally accepted ethical principles and culturally-dependent ones. By viewing ethics as a social contract, ISCT acknowledges the need for a communitarian approach in order to design truly effective ethical codes.

Furthermore, a 'minimalist' view of morality (based on general, universal principles such as justice) cannot stand apart from the particular social setting that helps qualify or elaborate its meanings according to a historical-cultural background (Walzer, 1992). To be sure, universal principles (or general ideas that all human beings would agree upon - e.g. rejection of torture, search for truth) can only provide a thin description of morality, necessarily embedded in the maximal (thick) accounts of different societies, which specify how these issues are interpreted and integrated into social practices.

Although it may seem the other way around, these community-based moral accountsoften a source of disagreement among countries- provide the true foundation from which a 
minimal morality can be extrapolated. Maximalism is the starting point; ethical frameworks can only grow out of each community's subjective moral understanding.

Cultural awareness must, therefore, be the first aim. Culture is a good theoretical concept to help identify the characteristics of particular communities, whether they represent organizations, industries or countries. As suggested earlier, the concept incorporates shared values and practices (cf. Schein, 1985, 1990). Since it provides the basis for community definition, it can also serve as the foundation for delineating the content and boundaries of the microsocial contract.

Investigation of the links between culture and social norm transgression may contribute to ISCT's framework by helping specify the culturally acceptable and unacceptable ethical principles. More importantly, since cultural values are hypothesized to provide community-members overriding principles that justify their "breaking" of social norms, an understanding of culture might lead to the establishment of ethical norms that are more compatible or sensitive to these values. This can be a good starting point for applying ISCT's concepts and hopefully generate more ethical understanding among different international communities.

Since the development of a multidimensional scale that includes a social contractarian dimension (assessing whether a particular behavior violates an unspoken promise or an 
unwritten contract), few empirical studies already supported the idea that community members do rely on an implicit social contract to evaluate the ethicality of particular actions (Hansen, 1992; Reidenbach \& Robin, 1990; Reidenbach, Robin, \& Dawson, 1991). Robertson and Ross (1995), for example, showed that salespeople were aware of their community norms and considered these ethical rules in judging their likelihood of giving an expensive gift or cultivating a friendship with a purchasing agent, while students (noncommunity members) did not. The former subjects were, however, more sensitive to jobrelated, situational factors such as size of the potential order, their current position against sales quota, and the probability of getting caught during the act. Thus, attention to community norms per se does not guarantee more ethical behavior. Instead of searching for a list of "temptations" that might deviate individuals from their group norms (potentially endless), what is needed is the specification of principles that can explain why other norms may be followed.

\section{A Cognitive Theory of Culture}

The conceptualization of culture adopted in this study has its roots in cognitive anthropology (ethnoscience), where culture is defined as a knowledge structure or as "a system of shared cognitions" (Smircich, 1983:348). This perspective emphasizes the similar mapping or organization of world events by members of a particular culture, according to a crystallized set of meanings, cognitions or thought processes. Culture is therefore a 'schema' 
or frame of reference that determines what to perceive, how to perceive and what to make out of these perceptions.

It is important to acknowledge the disparate views that permeate discussions of the culture concept. Although similarities in definitions may be encountered (e.g. in the learning and sharing of values and practices), there is not a dominant view of culture in either the anthropological or organizational literature. Yet, the cognitive perspective produced one of the most significant research streams, along with functionalism, symbolism and structuralism. Its distinctive focus is on the conscious processes carried out within the human mind.

The specific theory of culture chosen for this study is based on the work of Triandis (cf. $1972 ; 1989 ; 1990 ; 1993 ; 1994)$. From his initial examination of the 'subjective culture' (Triandis, 1972), which included elements such as shared attitudes, values, beliefs, norms, and self-definitions, the author developed a theory linking the self to its cultural context (Triandis, 1989), wherein he suggested that members of individualist cultures sample more the 'private self (cognitions that relate to the person- e.g. I am introverted; my qualities are few), while members of collectivist cultures sample more the collective (cognitions reflecting a collective view of the self- e.g. my family expects me to be introverted; my coworkers think of me as having few qualities) and public selves (cognitions about the generalized other's view of the self-e.g. people in general think I am introverted; people expect me to exhibit certain qualities). In his work, the constructs of idiocentrism and allocentrism are used as 
'psychological-level' correspondents of individualism and collectivism, i.e., they describe personality attributes that differentiate individuals within cultures (Triandis, Leung, Villareal,\& Clark, 1985). Individualist cultures have mostly idiocentric individuals, who tend to draw upon private cognitions; collectivist cultures, on the other hand, have mainly allocentrics, who tend to rely on collective and public cognitions. ${ }^{1}$

Contrary to the unidimensionality of individualism-collectivism postulated by Hofstede (1980), Triandis and his colleagues found that individual's private and collective cognitions are stored in different memory locations, therefore constituting separate cognitive structures (Trafimow, Triandis, \& Goto, 1991; Triandis, Bontempo, Betancourt, Bond, Leung, Brenes, Georgas, Hui, Marin, Setiadi, Sinha, Verma, Spangenberg, Touzard,\& Montmollin, 1986; Triandis, Bontempo, Villareal, Asai, \& Lucca, 1988; Triandis, McCusker,\& Hui, 1990):

"...people have a "cognitive schema" (a set of interrelated ideas) that includes individualist ideas and a separate one that includes collectivist ideas, and one can stimulate them to think individualist or collectivist ideas by priming them." (Triandis, 1993:170)

1 Both Individualism-Collectivism and Allocentrism-Idiocentrism are conceptualized and measured at the individual level. However, the former constructs are used to contrast people from different cultures (between-culture analysis), while the latter are used to compare individuals from the same culture (within-culture analysis). 
Examples of situations that induce differential sampling include: home vs. work or private vs. public situations, the type of group climate, presence of external threat (e.g., war), scarcity of resources, interaction with people from other cultures, and exposure to drugs. Individuals from the same culture, nonetheless, exhibit more of either individualist or collectivist ideas across a variety of situations:

"All of us carry both the individualist and collectivist tendencies; the difference is that in some cultures the probability that individualist selves, attitudes, norms, values and behaviors will be sampled or used is higher than in others." (Triandis, 1994:42).

Notice that in this theory both individual and group tendencies are acknowledged. Although the author is theorizing at the individual level (tapping at individual's cognitive structure), scores on individualism and collectivism represent a group's adaptation to a particular ecological system (see below) and as such, are continuously transmitted to individuals through different child-rearing patterns, and through other socialization processes (Triandis, 1990).

By theorizing at this level, the author favored a more parsimonious and less abstract (close to the data) conceptualization of culture, which allowed for the investigation of particular individuals that may deviate from the dominant cultural norms, the 
"countercultural" individuals (Triandis, 1994:42). The presence of idiocentric individuals in collectivist cultures (e.g. members of the upper class) and of allocentric individuals in individualist cultures (e.g. members of gangs, police officers) cannot be detected at the cultural-level.

According to this researcher, however, the analysis of individualism and collectivism at the cultural level is also worth pursuing. An investigation of the dominant norms, roles, and values can be done by treating respondents as 'key informants' (phrasing questions in a manner such as: "most members of your culture believe..."), therefore avoiding one of the main criticisms of Hofstede's work, i.e., using a mean score to represent a country's standing on a particular cultural dimension. Hofstede's procedure not only raises problems related to the representativeness of the chosen sample, but it requires reductionism, since the information about each country is subsumed under one single data-point (Kim, Triandis, Kagitcibasi, Choi, \& Yoon, 1994). Our procedure does not require this reductionism.

Keeping with Hofstede's (1980) data, individualism is high in North American and Northern and Western European countries, while collectivism is dominant in most of Latin American, African and Asian cultures, as well as in parts of Europe (e.g. southern Italy, rural Greece). Bell (1987) has estimated that about $70 \%$ of the world population belongs to collectivist societies. An understanding of how social behavior varies across these two dimensions can therefore expand scientific knowledge tremendously. 
Individualism is defined by an emphasis on the self as separate from others (coterminous with the body), and as an end in itself (Gould \& Kolb, 1964). Dominant or interrelated ideas include personal goals, self-reliance (here conceived as independence), selfdevelopment, autonomy, privacy, competitiveness, aggressive creativity, unrealistic interpersonal relationships, as well as the perception of the individual as the basis of social phenomena (Hsu, 1983; Lukes, 1973). In collectivism, the self often overlaps with a group (e.g., family). Consequently, the focus is on social norms and ingroup goals, interdependence, cooperation, self-sacrifice, obedience, and ingroup harmony. The group constitutes the unit of social perception, and individuals are seen as embedded in a universe of relationships.

Studies of values have identified clusters related to individualism and collectivism, with self-direction (creative, independent, imaginative, intellectual), stimulation (a varied life, an exciting life, daring), achievement (sense of accomplishment, ambitious, successful, capable), and hedonism (comfortable life, pleasure, happiness) representing the former, while security (national and family security, world of peace, inner harmony, sense of belonging), restricted conformity (obedient, polite, clean, disciplined), traditionalism (respect for tradition, devotion, accepting my portion in life), and prosocial (equality, social justice, helpful, forgiving, honest) better described the latter (Schwartz \& Bilsky, 1987; Schwartz, 1990).

In attempts to refine the constructs and the Individualism-Collectivism instrument (INDCOL- a set of attitude items initially developed by Triandis et al., 1985), Triandis and 
others investigated INDCOL's factor structure a number of times, consistently replicating a four-orthogonal factor solution. The factors of Family Integrity (e.g. "We should keep our aging parents with us at home") and Interdependence (e.g. "I usually feel that my relationships with others are more important than my individual accomplishments") characterized collectivism, while Self-Reliance (e.g. "I usually struggle through a personal problem by myself") and Separation/Detachment from Ingroups (e.g. "Children should feel honored if their parents receive a distinguished award"- reversed) described aspects of individualism (cf. Triandis et al., 1986, 1988, 1990).

Interestingly, these factor analyses showed that the main dimensions contrasting individualism and collectivism across cultures are Family Integrity and Detachment from Ingroups. In a review of such studies, Triandis (1990) concluded that these two factors explain differences between cultures, since members of collectivist countries valued living with their parents and extended family much more than members of individualist countries, who felt detached from their relatives. Within cultures, however, the factors of Interdependence and Sociability (e.g. "I like sharing little things with my neighbors") were significantly associated with allocentrics, while Self-Reliance and Competition (e.g. "Winning is everything") correlated more with idiocentrics. ${ }^{2}$ Therefore these empirical analyses show

${ }^{2}$ As a result of these studies, some items were deleted or changed, as part of the refinement process. The items here used as examples were chosen to reflect the latest form of the INDCOL instrument. 
that the parallelism between individualism-collectivism and idiocentrism-allocentrism did not provide identical characterizations.

In these factor-analytic studies, the key attributes of individualism and collectivism were identified by the following contrasts: broad concern for the family vs. relatively little concern for the family and relatives, emotional attachment to few ingroups vs. emotional detachment from many ingroups, relative attention to the view of others vs. lack of attention to the view of others, and tendency toward cooperation vs. tendency toward competition (Triandis et al., 1990). Although ideas such as self-reliance or conformity, or short-term vs. long-term perspective (which relates to the importance of tradition in one's life) are easily associated with individualism and collectivism, they did not discriminate members of different cultures well enough to be included in the above list of key attributes. The idea of selfreliance, for example, was important for both individualists and collectivists, but it carried different meanings for these two cultural groups (freedom to do individual's own thing vs. not being a burden to the ingroup).

The origins of individualism and collectivism are partially attributed to ecological patterns related to population density and geographic mobility. Densely populated and sedentary communities are usually collectivists (cf. Kim, 1994; Triandis, 1989). So the movement from hunting/gathering to agricultural societies was accompanied by a shift from protoindividualism to collectivism. Other important antecedents of individualism are cultural 
complexity and affluence: both factors, by providing a greater number of personal choices, tend to decrease individual's dependency on any particular group (Triandis, 1993). Therefore the advent of industrialization, as well as the more recent development of an 'information era', brought significant shifts towards a new form of individualism (Triandis, 1990). But collectivism is still dominant in most of the less developed countries, as a consequence of survival threats (limited availability of resources), common fate, and cultural homogeneity. Hence, societies' adoption of individualist or collectivist solutions can be seen as functional, since they constitute appropriate 'designs for living' (Triandis, 1989).

Different authors have acknowledged the positive and negative aspects of both designs (cf. Hsu, 1981). Consider, for example, the low gross national product per capita and the corruption in the political systems of some collectivist cultures vis-a-vis the low rates of heart attack per capita and the high degree of social support in difficult life situations. Individualist cultures, on the other hand, usually have greater GNP per capita and more reliable political institutions, but are confronted with high rates of stress, child abuse, juvenile delinquency, and suicide (Triandis et al., 1988; Naroll, 1983). Instead of defending the supremacy of a particular 'design choice', it is more advisable to move away from each extreme, by keeping the best contributions embedded in these options: 
"In short, only some aspects of social behavior can be governed by individualist principles (e.g. the marketplace). In many situations, such as behavior in the family, other principles are needed." (Triandis, 1990:51)

"We need to understand these constructs (individualism and collectivism) much better and move toward cultural forms that select the best elements of each cultural pattern and discard the remaining elements." (Triandis, 1993:162)

In spite of the commonalities that link collectivist and individualist societies across the globe, there are also cultural-specific aspects that deserve investigation. According to the country under consideration, individualist tendencies may include strong narcissism, competition, consultation to ingroups, and even anarchism. Similarly, collectivism differs on the emphasis placed on ingroups, on the degree of family integrity, obedience, and so on. Besides the degree of influence accepted from particular collectives, the number of groups that are influential and the breadth of their behavioral control is varied.

The importance of sampling individuals from different cultural settings is further justified by Hofstede's findings, which revealed many countries standing somewhere in the middle in terms of individualism and collectivism (e.g. Japan). There is already a significant amount of literature that examined these constructs across several countries or regions such 
as United States, Turkey, Greece, Italy, India, China, Japan, Egypt, Africa, and Latin America. One study in Latin America, for example, showed the high value that Hispanics place on "simpatia" (being nice and polite) in most public situations (Triandis, Marin, Lisansky, \& Betancourt, 1984). Other studies found that in "tight" cultures such as Japan, where even minor deviations from the appropriate behavior are sanctioned, individuals generally avoid telling what they feel (Iwao, 1988; Barlund, 1975), a finding probably less frequent in the relatively looser cultures of Brazil, China and Thailand. Also, in his analysis of India, Roland (1984) claimed the centrality of religious beliefs (e.g. peace of mind) in understanding the private self.

Triandis (1993) suggested that individualist and collectivist tendencies of individuals constitute 'cultural syndromes' or organizing themes (the centrality of the individual or the collective) that are reflected in particular types of individual perceptions, attributions, selfdefinitions, norms, values etc. The guiding principle is therefore either separation or connectedness. As summarized in Table 5, the distinction between individualists and collectivists is proposed as the underlying notion that links a number of elements of the subjective culture. There is already some empirical evidence showing that these elements are in fact significantly correlated among themselves (Triandis et al., 1990). 
As Triandis (1989)'s theory of the 'self in relation to the cultural context' advanced, the constructs of individualism and collectivism represent different cognitive schemata available to individuals. Entries on Table 5 therefore delineate the contents of such schemata. At the root or center of each cognitive configuration is what Markus and Kitayama (1991) have termed 'independent or interdependent construals of the self' (see entries under the category "Self-Definitions"). Typical members of individualist cultures (idiocentrics) have a conception of the self as autonomous, independent or separate from others, while most members of collectivist cultures (allocentrics) view themselves as part of an ingroup or social unit, i.e., embedded in a set of relationships. Direct empirical evidence comes from studies of the self-concept, which suggest that: 1) people in collectivist cultures define themselves predominantly in terms of social categories or ingroups ("I am a member of the Catholic Church" or "I am a son of..."), while people in individualist cultures see themselves as separate entities ("I am beautiful"; "I am a professor"); and 2) ingroups are perceived as more homogeneous than outgroups in collectivist cultures, whereas the opposite is found in individualist cultures (cf. Triandis et al., 1990). These studies also provide indirect evidence to the hypothesized distinction in social perception (first entry of Table 5): interdependent selves view the world in terms of groups or different relationship webs (and especially in terms of an ingroup-outgroup distinction); independent selves decompose social phenomena into separate individual entities, in much the same way as they see themselves. The centrality of the individual or the collective thus provide different units for perceiving and organizing world events. 
Kitayama, Markus, Tummala, Kurokawa, and Kato (1990) replicated a typical American finding in which the self is perceived as significantly more dissimilar to the other than the other is to the self (when reversing the question "Is self similar to other?" to "Is other similar to self?" subjects' ratings on perceived similarity increase). This finding is interpreted as a demonstration that American subjects, in general, have more distinctive and elaborated knowledge about themselves than about other people. Interestingly, the authors found an opposite (nonsignificant) pattern of ratings for Indian subjects, thus suggesting that collectivists may indeed have greater and more accessible knowledge about others than about themselves.

Triandis (1993) refers to an anthropological study of American and Japanese women conducted by Lebra (1984). She investigated the life histories of these two groups of women through a series of interviews and concluded that for American women, the self constituted the central figure with the social context providing the background; for Japanese women, the reverse occurred: "...most of the information was about relationships, and there was almost nothing about the self" (Triandis, 1993:174). This study indicates that collectivists may have fewer self-linked memories than individualists, a finding consistent with their ... "greater knowledge about the others" and with the importance that they place in social factors and achievement for the group. 
There is also theoretical and empirical support for the idea that collectivists experience much less cognitive dissonance than do individualists. In line with Doi's (1986) assertion that Americans have greater concern for consistency between the private and public selves than the Japanese, Markus and Kitayama (1990) explain that in a collectivist culture one's inner self (feelings, attitudes, opinions) has to conform to the demands of the situation, so that consistency may in fact be a sign of inflexibility or one's inability to adequately exercise selfrestraint. Triandis $(1989,1990)$ describes Iwao's (1988) comparative study of American and Japanese responses to scenarios in which the actor experienced some conflict with another person. In one of these hypothetical situations, a daughter brings home a prospective husband from another race. Among the possible responses, telling a lie ("think that he would never allow them to marry, but tell them he was in favor of their marriage") was the favorite answer for $44 \%$ of Japanese subjects, while only $2 \%$ of Americans chose that response. This finding indicates that the private and public selves are kept separate in collectivist cultures and that there is no pressure for consistency among them (see below Da Matta's 1993 and 1987 analyses of Brazil).

Studies of conflict resolution give support for entries 10 and 21(personal vs ingroup goals; harmony vs. confrontation) on Table 5. Collectivists tend to choose resolution strategies that are less adversarial (e.g. bargaining and mediation), while individualists prefer a more confrontational approach (cf. Leung, 1987). The former's emphasis on harmony is further attested in more lenient evaluations of group members, in greater display of social 
support, and in their subordination to ingroup goals (Bond, Chiu,\& Wan, 1984; Triandis et al., 1985; Nadler, 1986). Other studies also show that self-centered goals (such as "do whatever I think worth doing") and definitions for success (e.g. ability-based) are much more endorsed by individualists (Triandis, 1972; Tanaka, 1978) than collectivists.

Findings contrasting particular types of emotions exhibited by individualists and collectivists are summarized by Markus and Kitayama (1991). Ego or self-focused emotions (e.g. anger, pride) both express and promote one's independence and therefore are assumed to be more experienced by individualists. Few anthropological analyses have suggested, for example, that the feeling of anger is either inhibited or totally suppressed by collectivists, who fear disruption in social context. Consistent with this notion, a study by Miyake, Campos, Kagan, and Bradshaw (1986) showed that Japanese children were much slower than Americans in reacting to a mother's angry voice to stop moving towards an attractive object (a toy), suggesting less familiarity with this particular type of emotion. Other studies also found that the experience of anger was related to self and closely related others for American and Western subjects, while Asian subjects expressed anger only when strangers were involved (Matsumoto, Kudoh, Scherer, \& Wallbott, 1988; Stipek, Weiner,\& Li, 1989). Stipek et al. (1989) also found that Chinese subjects derived significantly less pride from their success than did Americans (see below the discussion of self-serving bias). These findings are in agreement with collectivist's emphasis on ingroup harmony and with the importance they give to ingroup needs and goals. 
There are also both theoretical and empirical accounts which claim deeper emotional ties between collectivist subjects (e.g. mother and child). The level of attachment among ingroup members, the fear of loneliness (see Table 5's entries under "Calamity") and the need for nurturance and social support are considered specially accentuated in collectivist societies (cf. Lebra, 1976). Considering the attention placed on 'the other', collectivists are expected to exhibit greater feelings of sympathy, communion, and shame (other-focused emotions). Working with Japanese subjects only, Kitayama and Markus (1990) for example, showed that those with a more 'interdependent construal of the self reported experiencing significantly more positive, ambivalent and negative other-focused emotions (e.g. Fureai-"Feeling of connection with someone", Tanomi-"Feeling like relying on someone", Omni-"Feeling of indebtedness").

Finally, a few interesting studies conducted in Japan have demonstrated that American's tendency towards self-enhancement (to give themselves higher ratings than they give to others) is reversed for collectivist subjects, who displayed a self-effacing or modestybias. Because of the self-enhancement tendency, Americans are expected to downplay unfavorable evaluations of the self and seek further information about their performance. Takata's (1987) and Wada's (1988) studies of self-evaluations showed that Japanese subjects, in contrast, were more confident in their ability level and requested less additional information after an unfavorable performance, as compared to a favorable one. These findings are further corroborated by Yoshida, Kojo, and Kaku's (1982) series of investigations of Japanese 
children, which demonstrated that the modest peer was not only perceived as having a more positive personality than the self-enhancing peer, but also for the older children, the former was also viewed as more competent!

Taken together, these studies suggest that humility may be the desired response in collectivist cultures (Markus \& Kitayama, 1991). This is a striking idea vis-a-vis the emphasis on impression management that individualists cultivate. Yet, modesty seems a natural response to the need to 'fit in', as opposed to 'stand out.'

As the entries under "Cognition" highlight, the search for uniqueness among individualists ("What makes me different") is contrasted with collectivists' demand for selfadjustment and conformity to ingroup needs ("what makes me the same"). Several studies conducted by Triandis and colleagues (cf. 1986, 1988) show that collectivists follow primarily ingroup needs and therefore believe in the importance of exercising self-control as a means of preserving ingroup harmony. The sense of individuality or self-esteem is derived from one's ability to develop and maintain certain relationships, which is largely a function of the degree of adaptability to the ongoing situational demands. Collectivists can therefore be expected to be higher in self-monitoring. Gudykunst, Yang, and Nishida (1987b), after developing Korean and Japanese versions of the self-monitoring scales, found that the US sample had lower means than these two groups (see entry 32 in Table 5). 
Consistent with Lebra's (1984) study of women's life histories, Shweder and Bourne (1984) found that in descriptions of their close acquaintances, Indians were much more situation-specific or attentive to their surrounding context (e.g. "He behaves properly with guests but feels sorry if money is spent on them") than were Americans, which provided context-free ("He is hesitant to give his money away") descriptions $46 \%$ of the time, versus only $20 \%$ for the Indians. Cousins (1989) replicated this finding with the use of an original version of the Twenty Statements Test (TST)- when asked 20 times the question "Who Am I?", Japanese students were more role specific and didn't rely on psychological traits as much as did Americans. However, when more contextualized self-descriptions were provided ("Describe yourself as you are with your family"), the inverse pattern was revealed: the Japanese made more generalizations about themselves than did the Americans.

A related study by Miller (1984) compared Indian and American causal attributions for other's prosocial and deviant behavior. She showed that overall, $40 \%$ of the American responses invoked general dispositions of the actor, a finding that confirms the 'fundamental attribution error' (Ross, 1977). Indian accounts, nonetheless, referred to these internal attributes only $20 \%$ of the time, while the majority of responses alluded to social or contextual factors, such as the actor's role. It may be that in collectivist cultures the pattern of causal explanation for other people's behavior is actually the inverse of the fundamental attribution error, with social factors such as norms and roles being implicated more heavily than attitudes and traits (see entries under "Attributions" in Table 5). 
Bontempo and Rivera (1992), in fact, showed that for studies that used the Fishbein and Ajzen's (1975) model in different countries (United States, Australia, England, Canada, Holland, Ireland, Israel, Spain, and Mexico), the more individualist the culture (according to Hofstede's 1980 index), the more attitudes vis-a-vis norms predicted behavioral intentions. Kashima, Siegel, Tanaka, and Kashima (1992) also found that Japanese subjects believed in the attitude-behavior link much less than did Australians.

In line with our discussion of the self-enhancement versus modesty bias, collectivists may also conform to a different pattern of attributions for success and failure. While Americans are known to exhibit a self-serving bias (cf. Martinko \& Gardner, 1987), making internal attributions for success (e.g., ability, effort), and external attributions for failure (e.g., task difficulty, luck), Kashima and Triandis (1986) found that Japanese students, after performing a culture-free intelligence test, used less of a coping strategy than their American counterparts, attributing success less and failure more to themselves. Shikanai (1978) also contrasted American and Japanese accounts after their participation in an anagram task. He showed that Japanese students attributed success mainly to the ease of the task and failure to the lack of effort. Although later investigations by the same author failed to confirm this pattern, ability seems less important than effort in Japanese explanations for success (Markus and Kitayama, 1991). Also, collectivists may engage less in personal face saving and more in saving face for the other or for the group (Ting-Toomey, 1988). I suspect that whistleblowing may be judged more negatively among collectivists than among individualists. 
In an early attempt to better define individualism and collectivism, Hui and Triandis (1986) asked psychologists and anthropologists from different countries to respond to a questionnaire describing ten target persons (e.g., spouse, mother, friend, coworker, neighbor, acquaintance, stranger) in seven situations, assessing concern for self-presentation, susceptibility to the opinions of others, amount of involvement in the lives of others, perceived correspondence between outcomes for self and others, and degree of sharing of material and nonmaterial gains. Respondents gave higher ratings for collectivists than to individualists in all these categories, indicating that the former view themselves as more intertwined in other people's lives. This sense of interdependence or embeddedness is contrasted with individualists' strong belief in "doing their own thing" and their perception of the world as constituted by separate or segmented pieces.

Individualists' feeling of independence is further evidenced in studies of values, which show their great commitment to the ideas of freedom, autonomy, achievement and so on. Schwartz's work on value structures clearly reveals a dichotomy between these values and the values of security, obedience, duty, etc, which reflect collectivists' dependency on ingroups (cf. Schwartz, 1994). The factor-analytic studies performed by Triandis and colleagues (1985, 1986, 1988, 1990) strengthened these ideas by repeatedly affirming collectivists' emotional attachment to ingroups, as well as their concern for family integrity. Individualists on the other hand, felt detached, paid much less attention to the view of others, and showed less concern for family and relatives. 
Triandis has claimed several times (cf. 1972, 1989, 1990) that collectivists are especially attentive to the ingroup-outgroup distinction, i.e., they alter their behavior according to who is 'the other' -e.g. a friend versus a stranger (see entries on "Social Behavior"). A study by Gudykunst, Yoon and Nishida (1987a) confirmed this expectation by comparing Korean (very collectivist), Japanese (somewhat collectivist) and American (very individualist) students' interactions with their classmates (ingroup members) and with strangers (outgroup members). The two types of interactions were different along the attributes of personalization, synchronization and difficulty to the greatest degree for Koreans, and more so for Japanese than for American students. Also, for both collectivist cultures, interactions with ingroup members were more personalized (intimate, deep, broad, spontaneous, flexible, smooth and satisfying), more synchronized (effortless, well coordinated) and less difficult than for the individualist culture.

Differences in how individualists and collectivist view ingroups (see entries on "Ingroups") help us understand much of their social behavior. Individualists belong to many ingroups and therefore give less attention and are less susceptible to the influence of each one of them. They perceive their ingroups as more heterogeneous than their outgroups (cf. Lee and Ottati's 1990 comparison of American and Chinese subjects); in fact, individualdifferences are valued within closer relationships and can be disputed in an atmosphere of open confrontation. Collectivists have, on the other hand, few but very close ingroups, which 
exert much more pressure towards homogeneity of thoughts and actions and maintenance of ingroup harmony/stability.

Because of this sense of interdependence among ingroup members, it takes time for newcomers to develop the level of intimacy needed to feel like they belongs (entry 26). For individualists there is more freedom to get in and out of groups, since there is less expectation and more superficiality in relationships. A study by Gudykunst and Nishida (1986) confirmed that, overall, collectivists had more intimate and pleasant relationships than individualists. However, it is important to specify the types of relationships that are more important for each culture. These authors found that Japanese subjects displayed greater intimacy towards friends, acquaintances, colleagues and coworkers, while Americans were more intimate towards a lover, fiancé, son, sister, roommate and neighbor. Consistently, Atsumi (1980) has argued that the more distant the relationship (e.g., with a stranger), the more Japanese behavior will resemble the American pattern of cost-benefit analyses and generalized public norms. According to the author, Japanese behavior towards coworkers is governed by a combination of norms and cost/benefit considerations.

Another study by Bontempo, Lobel, and Triandis (1989) presented Brazilian and American subjects with scenarios describing a set of 'costly' prosocial behaviors (e.g. be a full-time caretaker at the hospital, or to loan money) towards either a family member, a friend, a coworker, a neighbor or a fellow national. Subjects were assigned to a public or 
anonymous condition, and were asked what they would do in the particular situation (behavioral intention), what was expected of them (perceived norm), and how they felt about it or how enjoyable it was to perform the behavior in question (affect). Findings indicated that the Brazilian sample showed more willingness to do what was expected of them and also reported greater joy in doing so than did the Americans. Brazilian responses also did not vary along the public vs. anonymous condition, whereas Americans complied more with what was expected of them when their actions were visible to others. The authors concluded by suggesting that Brazilians (as well as other collectivists) might have internalized their ingroup norms (behavioral expectations are made consistent with personal values) to such an extent that it is pleasant to do what is expected of them. I further speculate that Brazilians experienced affect not derived from engaging in these particular behaviors, but from focusing on the 'joy' that ingroup relationships provide. In that respect, 'costly' or time consuming behaviors can be viewed as an opportunity to strengthen social ties (see below discussion on communal vs. competitive social exchanges).

Another evidence of collectivists' internalization of ingroup norms is provided by Earley (1989) who shows that 'social loafing' (reduction of group's output when individuals' contributions are not clearly identifiable) occurs among Chinese only when they are working with outgroup members. When Chinese subjects are working with ingroups (family, friends, relatives, co-workers) they do not decrease their individual levels of performance, thus 
respecting ingroup norms without supervision. This is one more indication that the ingroupoutgroup distinction is crucial for collectivists.

Forgas and Bond (1985) had collectivist (Hong Kong and Chinese) and individualist (Australian) subjects respond to multidimensional scaling judgments of 27 social episodes (e.g., arriving late for a tutorial). The dimensions that emerged were interpreted according to pairs of semantic differential scales such as equal-unequal, pleasant-unpleasant, communalisolated, cooperation-competition etc. Out of the four derived dimensions, two were culturespecific, reflecting for Hong Kong and Chinese subjects the importance of communal feelings, inequality of power, and the relationship aspects or qualities that characterize ingroups vis-avis outgroups, such as intimacy and pleasantness (see below for a discussion of the link between collectivism and power distance). The Australian-only dimension referred to competitiveness and feelings of being in control of the situation (relaxed-anxious; selfconfident-apprehensive). Therefore collectivists' social construction of meaning emphasizes communal exchanges/relationships and power, while individualists' strong notion of competition and control is more akin to the idea of social exchanges in the marketplace or social contract regulation. This finding is consistent with Triandis's (1990) comparison of individualist and collectivist exchanges. Whereas individualists exchange resources of equal value, for a short time duration and under a contract agreed in advance, collectivists often exchange nonmaterial resources of ambiguous value (e.g., reciprocated services), implicitly 
agreed between parts and expected to last for a long period of time (sometimes as long as the relationship).

The importance of trust in collectivist cultures is noteworthy. Individuals rely on each other's words to get things done. It is a system of favors that works through frequent reciprocation, feelings of solidarity, and constant strengthening of relationship quality. If there is lack of trust (e.g., with outgroup members), individuals may, however, show great hostility and behave even more competitively than individualists (Triandis, 1989).

Ingroup behaviors are learned early in socialization. Triandis (1990) reviewed studies of child-rearing patterns that show obedience, duty, and dependence towards authority figures as the key socialization messages delivered by collectivist parents, while individualist families stress self-reliance, independence and a strong sense of privacy (vertical relationships may be more important than horizontal for collectivists, e.g., giving in to parents' wishes; the reverse is true for individualists). Collectivists' families establish a great degree of bondage, and mate selection is geared to preserve family integrity, while individualists choose partners on the basis of their physical attractiveness (Dion \& Dion, 1988; Dion, Pak,\& Dion, 1990).

The centrality of the family in collectivist cultures can also be inferred by the amount of social status that it provides. Individuals may be treated with great respect because they belong to a specially wealthy or honored family or because their families are connected to 
somebody considered 'important'. Collectivists thus may inherit certain attributes, contrary to individualists, who usually have to work pretty hard to deserve them (ascribed vs. achieved attributes).

Individual's identity, therefore, varies along the individualism-collectivism dimension. Whereas in the former, identity is more a function of what one has achieved (possessions, accomplishments, experiences), in the latter it is rather defined by a particular set of relationships (family, friends, neighbors etc.). Consistently, the qualities or attributes valued in a person are also different, with the former's emphasis on rationality, predictability and task orientation, against the latter's appreciation of emotions, spontaneity and people's orientation. The American historian Richard Morse (1988) alludes to the Brazilian story "O Alienista" by Machado de Assis, in which a scientifically rigorous doctor imprisons $80 \%$ of the local population in a mental hospital for not fulfilling all the necessary mental health requirements. At the end, the doctor however realizes that a rational and balanced individual -- like himself - must be the real crazy person; he releases all of his patients and decides that he is the one that needs mental treatment.

In order to properly relate to an individual in a collectivist society, one has to understand his/her position in the social structure, because individuals are treated differently and are given unique rights/favors according to where they stand in the hierarchy. The Brazilian anthropologist Da Matta (cf.1993) explains that the sentence "Do you know who 
are you talking to?" is frequently used by Brazilians as a reminder of one's right to receive special treatment. Collectivists' hierarchical view of society is corroborated by Hofstede's (1980) finding that collectivism is highly correlated $(r=67)$ with power distance (the perception that those at the top of the hierarchy are very different than those at the bottom). Because individuals are not viewed as equals, what is good for a certain person may not be good for the other, specially when there are large power differentials (collectivists behave consistently with the principle of 'limited good'- if a good thing happens to an outgroup member it is probably bad news for the ingroup). I suspect this is due more to the perception of a power struggle than to that of a 'zero-sum' situation of unavailability of resources (an economics perspective better describes individualists' view of the world).

Contrary to collectivists' hierarchical conception of society, individualists hold an egalitarian view highly sustained by equity principles ("to each according to his/her contributions"). Individuals rights and obligations are the same irrespective of their social position, and people are more identified by what they do, as opposed to where they belong in the hierarchy. In line with these ideas, Marin (1985) reviewed a series of resource allocation studies and concluded that there is considerable evidence suggesting that individualists choose equity, while collectivists prefer equality and need as the norm for distribution of resources, especially when resources are unlimited. 
Taken together, the findings summarized in Table 5 are consistent with studies on morality that compare individualist and collectivist cultures. First, moral judgments are more dependent on the situation, on the actor/actress's social position and especially on his/her ingroup-outgroup status in collectivist societies, vis-a-vis individualist ones (Hamilton \& Sanders, 1983). Da Matta's (1991) analysis of the Brazilian maxim "for friends everything; for enemies the law" is illustrative of the supremacy of ingroups' system of duties and personal ties over more institutionalized arrangements. The bulk of collectivist's social transgression may in fact be a function of ingroup favoritism, i.e., whenever ingroup members are involved, the norms of ingroup preservation take precedence over the law. Also, collectivists may be more willing to accept social norm transgression according to the context and the particular individual(s) engaged in it (e.g., after winning the soccer's world championship, the Brazilian delegation was authorized by immigration officials to reenter the country without paying excess fees over the $\$ 500$ individual import quota).

Similarly, Ma (1998) suggested that a Chinese perspective on Kohlberg's model of moral development $(1976,1981)$ may differ from a Western perspective, in particular at stages 4,5 and 6 . The Western/Individualist perspective is notably rational (with its costbenefit calculus), moving from the strict consideration of the law towards more elevated (autonomous but socially-oriented) self-principles. The Chinese moral stage evolution is rather emotion-based, moving from affective and person-oriented responses (legally loose) to more altruistic and, finally, ethically neutral judgments. 
An important study by Haidt, Koller, and Dias (1993) contrasted Brazilian and U.S. adults and children in their responses to scenarios describing harmless yet offensive norm violations (e.g. cutting a national flag in pieces and using it to clean the bathroom; eating your own dog after it was killed in a car accident). Findings showed that in both countries, college students at elite universities (the more individualist subjects) viewed these stories in terms of social convention or personal preference, while other participants, especially Brazilians with low socioeconomic status (the more collectivist subjects), judged them as immoral. For the former subjects, moral judgments were strongly associated with appraisals of harmfulness, whereas for the latter, they were better predicted by how individuals felt about it (affect). These results confirmed the authors' expectations that American subjects (the most "permissible" in their evaluations) would conform to a Western, harm-based (utilitarian) view of morality, while Brazilian subjects would tend to infer morality beyond harm. They also support Ma's (1988) previous discussion of a rational/cognitive versus an emotional perspective.

Another important finding of this study was that collectivists subscribed to what Shweder (1990) called 'ethics of community', that is, their moral thought and discourse were based on the rules that govern relationships (duty, respect, obedience), as well as on the understanding of one's social role. After coding about 1,620 subjects' justifications for judging actor/actress behavior in the particular offensive stories as "very wrong, little wrong or perfectly ok", the authors found that the groups that took a fully moralizing stance 
(classifying the action as universally wrong and punishable), made more reference to the social categories that relate to what one ought to do in relationships. Philadelphia college students (the least moralizing or the more permissive respondents), on the other hand, endorsed significantly more than any other group what Shweder (1990) referred as 'ethics of autonomy'. Their justifications evoked rights, justice or freedom of choice (and assessments of potential harm). Moral judgment for these subjects responded to the basic regulating principle or individual need to increase autonomy, choice, and control. Note that both of these 'codes of moral thought' are associated with an interdependent (ethics of community) or independent (ethics of autonomy) view of the self.

Unfortunately, current scientific knowledge on morality is mostly based on the 'autonomy' moral code, drawing upon either Kohlberg's (1981) or Gilligan's (1982)'s individually-oriented models of moral reasoning. As Miller (1994) explained, both of these approaches rely on individualist assumptions that reveal an individual-centered, contractual/universal, dualist, rights-based, and decontextualized moral perspective. This perspective either ignores (in Kohlberg's case) or downplays (in Gilligan's case) the importance of interpersonal responsibilities related to one's social position or role in collectivist cultures, which according to Miller's empirical work, not only carry the status of moral obligations, but can be seen as even more important than justice considerations. 
Cross-cultural work on morality calls attention to the significant effect that cultural assumptions have on the moral reasoning process. The practical implication of this work is that in order to design ethical organizations, one has to seek how particular cultural patterns assist moral regulation. Because culture gives the basic code of moral thought, the overriding principles that regulate and predict responses to more specific sets of norms, successful corporate ethical codes need to evolve from this understanding. Considering that the specific aspects of corporate code design and implementation are under the control of management (e.g., the extent of employees' involvement in the design process; the amount of training provided), the analysis of causal attributions for unethical behavior can help in devising better methods for increasing employees' compliance with these codes. 


\section{CHAPTER 3}

\section{RESEARCH METHODOLOGY}

\section{Research Questions}

The present study addresses two main questions:

1. Do individualist and collectivist attitudes affect individuals' attributions for unethical behavior?

2. What types of attributions are predicted from individualism and collectivism?

I begin by acknowledging that each ethnic group within a particular country specifies for itself the ethical principles to be incorporated in its local community practices. In the language of Integrative Social Contracts Theory, the ethnic group, our unit of analysis, sets the terms of its microsocial contract, while simultaneously agreeing to abide to a broader set of ethical principles (the macrosocial contract). Notice that the principles of ISCT can be applied at multiple levels of analysis. So, in an international context, countries represent local communities which abide to an international macro contract with enough freedom to specify their national norms. Within each country, community members conform to general norms, while safeguarding the right for subcommunities (e.g., organizations, industries, or ethnically 
diverse groups) to establish more specific guidelines for their activities. To be sure, the microsocial contract can be investigated at different levels, but there is only one hypothetical macrosocial contract.

Although ethnic groups could potentially be studied by examining the different aspects of their subcommunities (a differentiation view), this study instead chooses to provide a broad picture of their cultural background which emphasizes their similarities (an integration view) (Meyerson \& Martin, 1987; Martin, 1992). The identification of broad cognitive patterns is one way to study cross-cultural differences.

\section{Why Anglos and Hispanics}

The Anglo and Hispanic communities were chosen for comparison, given their expected opposition in scores of individualism and collectivism. Although Hispanic groups living in United States are likely to be more individualist than Hispanics living elsewhere (e.g., in Latin America), they are still probably less individualist and more collectivist than Anglos.

A quick examination of the number of journal articles in the psycLIT database which address these two ethnic groups demonstrates the tremendous growth of interest in the topic of ethnic diversity, in such different fields of inquiry as medicine, biology, psychology, and sociology. This growing interest is also present in the business literature, due to major 
changes in the demographic composition of the American workforce (greater influx of Hispanics and African-Americans, as well as women).

Management research on diversity has typically examined the effects of individual variables (e.g., sex, race) on attitudes and behaviors of employees (Riordan \& Shore, 1997). The potential to impact a number of organizational outcomes (e.g., job satisfaction, employee morale, group creativity) has raised serious concerns with respect to the effective management of diversity. The author hopes to address some of these concerns by examining how ethnic diversity can affect a set of postulated relationships.

A note of clarification is important. Although there is a great deal of confusion concerning the meaning of the term ethnicity (cf. Phinney, 1996), it is mostly agreed that it refers to a multidimensional construct which denotes race or national origin, culture (the values, attitudes and behaviors of a particular ethnic group), and ethnic identity (a sense of group membership). This study does not make any distinction among these three dimensions, using them interchangeably. 


\section{Hypotheses}

The following hypotheses will be investigated by the present study:

H1: Individualist and collectivist attitudes will predict attributions for unethical work behavior, in such a manner that:

H1a: Individualist attitudes will lead to a market-oriented type of attribution response pattern, which is characterized by an impersonal/ calculative, general /rules-based and self-reliant/ autonomous assessment of ethical situations;

H1b: Collectivist attitudes will lead to a relationship-oriented type of attribution response pattern, which is characterized by a personalized/ emotional, particularistic/ driven by favoritism, and paternalistic/ familial assessment of ethical situations.

H2: Anglos and Hispanics will differ in the strength of individualism and collectivism predictions, in such a manner that:

$\mathrm{H} 2 \mathrm{a}$ : The relationship between individualist attitudes and a marketoriented attribution response pattern (MO-ARP) will be stronger for Anglos; 
$\mathrm{H} 2 \mathrm{~b}$ : The relationship between collectivist attitudes and a relationship-oriented attribution response pattern (RE-ARP) will be stronger for Hispanics.

These hypotheses were developed to explain individual responses given by Anglo and Hispanic business graduate students to a series of hypothetical scenarios that describe unethical situations within the workplace (see next section). They address the expected dominating influences of individualism or collectivism on members of the Anglo and Hispanic communities, respectively.

Hypothesis one reflects the main expectation that individualism and collectivism will influence individuals' attributions for unethical work behavior. Findings reviewed in Table 5 under the label "Attributions" (entries 2-4) provide strong support to the idea that individualism and collectivism affect attribution patterns. Hypotheses $1 \mathrm{a}$ and $1 \mathrm{~b}$ further elaborate on how these independent variables are expected to impact attribution responses. As explained below, these hypotheses can be derived from two central contrasts: the idea of an interdependent versus independent self and their corresponding hierarchical versus egalitarian worldviews.

The personalized/emotional vs. impersonal/calculative type of attribution response is supported by entries number $5,16,17$ (personalized relationships vs competition, fair 
exchange), 26, 27, and 28 on Table 5. Ethical issues are expected to be personalized by members of a predominantly collectivist culture because they tend to view themselves as embedded or part of social situations. Behavior, therefore, is not regulated by objective or formal criteria, but rather by relationship norms and emotional ties. Individualists, on the contrary, see themselves as separate from the social context and consequently tend to apply formal, impersonal, cost-benefit rules to assess situations.

The particularistic/driven by favoritism versus general/rules-based type of attribution response is supported by entries 17 (hierarchy, duty and ingroup harmony vs individual achievement, competition and fair exchange), 23, 25, 27, and 28 (ingroup vs generalized public norms) on Table 5. Collectivist's hierarchical view of society is specially exemplified by the ingroup-outgroup distinction, because only ingroups are perceived as equals, while outgroups are seen as largely untrustworthy. The perception of a power struggle leads to behavior aimed at ingroup preservation, which produces favoritism. In a more egalitarian society, universal principles are better accepted and endorsed, because individuals are judged as equals. Since individualist societies are characterized by small power differentials, there is less hostility and skepticism in evaluations of outgroup behavior.

Finally, the idea of hierarchy and ingroup preservation, combined with that of interdependence or embeddedness in relationships, explain collectivists' expected paternalism in attribution responses. This is contrasted with individualists' strong tendency to rely on their 
own capabilities. The two types of attribution response builds on findings from entries 5,15 , 16, 17 (obedience, duty vs. autonomy, freedom), 18, 21, 23, and 30.

Table 6 summarizes the expected attribution response patterns for individualists and collectivists. Each contrast (e.g., personalized vs. impersonal) is seen as a separate dimension, or attribution response category. The main basis for category \#1 is the distinction between interdependent vs. independent self, or what Markus and Kitayama (1991) have called 'divergent construals of the self. These construals or self-regulatory schemata address how individuals see themselves in relation to others. As the foundation for the self-system, they also help regulate intrapersonal (e.g. motivation) and interpersonal processes (e.g. social perception). The distinction between hierarchical vs egalitarian worldviews, which is the main support for response category $\# 2$, captures the essence of how individualists and collectivists relate to groups: in the former, behavior is regulated by general, universally applied principles or rules, while in the later it is dictated by the ingroup-outgroup distinction and varies accordingly. The last category (\#3) combines the two rationales that regulate both self and group behavior. Self-reliance, autonomy or freedom of choice are fundamental rules for individualists not only because of their independent view of the self but also because of the perception that all individuals are equals. Similarly, collectivists' paternalism or familial orientation is as much based on a sense of interdependence as in a hierarchical view of society. 
Together, the attribution response categories form an attribution response pattern which I call either relationship-oriented or market-oriented. While in the former the emphasis is on interpersonal exchange, the latter is best described as contractual or transaction-based. According to hypothesis one, these two types of attribution response patterns can be predicted from individualist and collectivist attitudes.

Although hypothesis one is expected to hold across the two ethnic groups chosen for comparison (Anglos and Hispanics), it is plausible to assume that the relationship between individualism and market-orientation will be stronger for Anglos, while the relationship between collectivism and relationship-orientation will be stronger for Hispanics. Hypothesis two is supported by Triandis' theoretical and empirical work relating the self to its cultural context (see pages 39-40), wherein individualistic or collectivistic cognitions, as well as values, attitudes and so on, are shown to be sampled from an individual's mind with higher or lower probability, depending on his/her cultural background. For each ethnic group therefore, one set of attitudes (individualist or collectivist) will have greater centrality.

According to this theory, however, different situations (as introduced by each scenario) can also affect this cognitive sampling process. It is possible that hypothesis two will not hold across all scenarios, because a situation involving paternalism, for example, may induce equally strong collectivist predictions for both groups. 


\section{Sampling and Measurement Procedures}

Cross-cultural differences in attributions for unethical work behavior will be tested with a sample of Anglo and Hispanic business graduate students from two large state universities. These students will be presented with the same set of scenarios which: 1) introduce an ethical issue at the workplace - e.g., bribery, conflict of interest, paternalism; and 2) conclude with an unethical decision from the part of those involved in the situation - e.g., top management, a marketing analyst, a general manager.

These scenarios were originally developed by Fritzsche and Becker $(1982,1983)$ to include five categories of ethical problems most commonly cited in cases from business ethics books, as well as from material of the Summer Institute on Ethical Issues in the Management of Public and Private Institutions (Dill, Donaldson, Goodpaster,\& May, 1979). These categories covered issues that practitioners listed as unethical practices of primary concern, such as bribery, dishonest advertising, and whistleblowing (Baumhart, 1961; Brenner \& Molander, 1977). The five categories 'coercion and control' (when a manager is pressed by an external source to make a particular decision), 'conflict of interest' (when a manager's personal interest may harm the firm), 'physical environment' (a special case of conflict of interest in which the affected party is the physical environment), 'paternalism' (when a manager is faced with the need to balance individual autonomy with consumer welfare) and 
'personal integrity' (when a manager is dealing with issues of conscience) guided vignette construction, with two vignettes per category forming the initial instrument.

In later versions of the instrument, the number of vignettes was reduced to five- one vignette per category, following considerations of construct validity (Fritzsche \& Becker, 1984; Becker \& Fritzsche, 1987), and subsequently to four (Fritzsche, 1988), so as to restrict their content domain to marketing-related issues. In the latest published work using these scenarios (with minor modifications), Maher and Bailey (1994) also selected marketingoriented vignettes in order to increase consistency across scenarios, thus limiting them to a specific context.

Scenarios or vignettes are among the most popular measurement techniques in business ethics research (Weber, 1992). They introduce "short descriptions of a person or social situation which contain precise references to what are thought to be the most important factors in the decision-making or judgment-making processes of respondents" (Alexander \& Becker, 1978:94, as in Weber, 1992). According to Cavanagh and Fritzsche (1985), the main advantages of scenario use include the possibility of presenting complex and detailed issues that can be standardized across respondents, and of manipulating multiple variables, through small variations in vignette content. Cross-study comparisons can also be made, if scenarios are replicated in a consistent basis. Important limitations inherent to the technique are its possible lack of realism and external validity, given the difficulty in generalizing research 
findings to other situations (scenarios are by definition situation-specific). Also, in a critical assessment of scenario research, Weber (1992) identified the main problems associated with the use of this technique: lack of a theoretical framework that could guide hypothesis-testing and the absence of any method to allow comparisons across vignettes (the vast majority of empirical analyses address each vignette separately). The author further criticized the scarcity of validity or reliability checks, the frequent report of low response rates, the use of student samples, and the often ignored issue of social desirability (although he mentioned an empirical study by Armacost, Hosseini, Morris and Rehbein (1990) which found less social desirability bias in scenarios than in other research techniques).

In line with Weber's (1992) critique, Fritzsche and Becker's (1983) vignettes can be positively evaluated along the following aspects: they were pretested to assure relevancy in the selection of ethical issues, they were modified to increase construct validity, there was an effort to link them to ethical theories (Fritzsche \& Becker, 1984) and, although with significant instrument variation, some of the vignettes were consistently investigated across studies (see also Becker \& Fritzsche, 1987; Fritzsche, 1988). However, and in spite of "situational ethics" - responses typically varied across vignettes/categories, there was no systematic way of linking across-scenario responses into a more unified pattern of thought that could account for individual's moral reasoning process (Weber, 1991). As a consequence, it was difficult to state a priori what differences in scenario responses one might 
have expected. Another limitation in the scenario responses is that they indicate behavioral intentions (what one would do in a hypothetical situation), as opposed to actual behavior.

The paragraphs that follow review the empirical evidence on the vignettes chosen for this research. Studies by Fritzsche and Becker $(1982,1983,1984,1987,1988)$ used samples of students and marketing managers. Also, American managers were compared to French and German counterparts in the 1987 study. In all of these empirical examinations, respondents varied their degree of acceptance of unethical behavior according to the particular ethical dilemma presented in the vignette categories. In the 1983 study of marketing managers (with the original set of 10 vignettes), responses differed in the coercion and control, personal integrity, and conflict of interest dilemmas, being least ethical in the first and most ethical in the last category. The paternalism category was not significantly different than the conflict of interest and personal integrity categories. Also, within each category, respondents were more ethical the riskier the situation (e.g. less likely to pay a bribe, versus accepting pressure from a supplier).

In Fritzsche's 1988 investigation, marketing managers were also found more likely to pay a bribe (coercion and control) and secondly not to blow the whistle in a situation of possible physical injury for others (personal integrity), as compared to yielding to a personal interest or lying in an advertising campaign. Responses were also affected by the magnitude of consequences and the role of the decision-maker as actor or requestor, the two 
independent variables which were manipulated through different scenario versions. As the Becker and Fritzsche's 1984 study confirmed, managers seemed to engage in utilitarian ethics, weighing the situations presented in the vignettes in a cost/benefit fashion. The authors take this finding as evidence of the "...role of economics in managerial decision making" (Fritzsche \& Becker, 1984:174). In this study, open-ended reasons for endorsing a particular course of action were largely categorized as utilitarian (or calculative); interestingly, the few rights or justice-based responses were linked to more ethical behavioral intentions.

In the 1987 Becker and Fritzsche cross-cultural examination, American, French and German managers responded similarly to certain scenarios (they were unlikely to sign a higher-priced contract in the conflict of interest vignette, or reluctant to publish a book with instructions on how to build a bomb in the paternalism vignette), but differed significantly in the three other vignettes: American managers were less likely to engage in bribery or to approve environmental pollution. Together with French managers, however, Americans were also less likely to blow the whistle in a situation involving possible physical injury (personal integrity vignette).

It is noteworthy that among the empirical evidence provided by Fritzsche and Becker, there is good indication that American managers reason according to an utilitarian perspective and demonstrate special concern with the legal implications of their actions, as compared to 
French and German managers (cf. Fritzsche, 1988). These findings replicate research in cross-cultural psychology and are in the direction of this study's hypotheses.

In a couple of studies using slightly modified versions of Fritzsche and Becker's vignettes (Lysonski \& Gaidis, 1991; Maher \& Bailey, 1994) findings failed to confirm the researchers' expectations concerning: 1) cross-cultural differences among business students in US, Denmark and New Zealand (see Table 3 for a summary of Lysonski and Gaidis' study) and 2) perceived gender differences among two samples of marketing alumni. In this later study, Maher and Bailey (1994) manipulated the sex of the actor/actress involved in the scenarios and found that, for both male and female subjects, attributions for unethical behavior did not differ with gender. The authors investigated the attribution dimensions of stability and locus of causality (Weiner, Frieze, Kukla, Reed, Rest, and Rosenbaum, 1972), and the open-ended types of attributions respondents gave to the unethical decision added at the end of each scenario.

Although carefully designed, this last study constitutes a good example of how the specificity of scenarios may lead to difficulties in addressing a particular research question. To be sure, the alleged stereotypes of women and men as either influenceable or aggressive (respectively) would probably be more easily detected in a sexual harassment scenario, for example. However, the lack of evidence of gender bias in certain business ethics situations 
says something about the importance of context in understanding ethical behavior, a conclusion that is reinforced by Fritzsche and Becker's 'situational ethics' findings.

Furthermore, this conclusion reinforces the need to ground empirical investigations in a well developed theoretical framework that can guide hypothesis construction and testing (in this case through a selected number of scenarios). For example, how are United States, Denish and New Zealand business students expected to differ in their responses to business ethics scenarios? Why? Can a rationale for these expectations be provided? Why should a particular set of scenarios be used?

Aside from their methodological strengths, Fritzsche and Becker's vignettes were selected for this study because they were developed closely in line with the special concerns of American business practitioners, thus mapping, at least to some extent, the type of business environment that is relevant for business graduates in South Florida. Not surprisingly, these scenarios reflect, in broad terms, a harm-based or individualist view of morality (that is, unethical behavior is associated with some form of physical, economic and/or psychological harm).

Among the original set of ten vignettes, this study will use the five vignettes (one per category) that provided good discriminant validity in earlier examinations (Fritzsche \& Becker, 1983). These vignettes were kept in most of the subsequent studies and seem to 
represent a marketing-oriented domain, perhaps with one exception, i.e., the paternalism vignette. Another scenario which describes a hiring situation with important implications for competition assessment - a marketing analyst is hired on the basis of his knowledge of a competitor's cutting edge in software development - was also included.

Following Maher and Bailey's (1994) examination, I investigate the types of attributions respondents provide for unethical decisions added at the end of each vignette (e.g., paying a bribe, polluting the environment). For the four vignettes used in their research, I adopted their suggested modifications in content (in order to clarify scenario descriptions) and the final vignette decisions created by these authors, whenever available (Bill Smith and John Ward's vignettes). For the rest of the scenarios, original decisionoutcomes were formulated.

The idea of asking respondents to evaluate a particular decision, as opposed to asking them what would they do in the vignette situations, is an interesting way of avoiding the possible social desirability bias in respondents' stated behavioral intentions. One may speculate that Fritzsche and Becker's finding of a consistently low endorsement of unethical behavior by marketing managers (cf. Fritzsche \& Becker, 1987) is due to such bias, instead of a true managerial sensitivity to ethical practices. Also, by operationalizing causal accounts in terms of attributions for other people's behavior, this study further controls the problematic acceptance of wrong doings (Randall \& Gibson, 1990). 
Addressing Weber's (1992) request for a systematic way of linking results across vignettes, this study puts forth a set of attribution responses for each scenario that test an underlying moral reasoning pattern that is consistent with individualism and collectivism. Within each vignette, respondents are asked to justify why the actor(s) chose an allegedly unethical decision by rating how much they agree or disagree with six attribution statements (further extended to eight, after pretest). These statements tap into each content domain of the hypothesized attribution response categories (see Table 6). Although they were worded to suit the specific scenarios, they recreate the same underlying theme (e.g., personalized/ emotional or impersonal/calculative type of response), and, as such, represent different ways of testing each attribution response category. The attribution responses were jointly developed by the author (whose nationality is Brazilian) and an American doctoral student.

Also in agreement with Maher and Bailey's (1994) empirical examination, respondents are asked to rate the degree of ethicality and acceptability of each decision. Although scenario decisions were developed according to Fritzsche's (cf. 1988) previous suggestions of what might constitute potentially unethical behavior in each vignette's case (e.g engaging in bribery), the inclusion of ethicality ratings is in itself an acknowledgment that individual or cultural perceptions vary in terms of morality assessments. This study hence allows a direct comparison of differences across the two ethnic groups in the degree of permissibility in moral judgments, through both ethicality and acceptability ratings. Further evidence of 
permissibility can also be inferred from respondents' frequency ratings at the end of each scenario, which indicate perceived actual practices in their particular industry.

\section{Survey Instrument}

The questionnaire of individualism and collectivism (INDCOL) will be combined with the six vignettes into a single survey to be administered to business graduate students at two state universities in the southeast. Although their campuses are not far from each other, they are located in different cities and their student population is quite distinct: the southern location is more multicultural (with a Hispanic majority) while the northern campus is largely composed of Anglo students. The final sample should therefore be somewhat balanced between the two ethnic groups of interest. Since master's degree classes are exclusively held in the evening, most of the students are expected to be currently working.

The survey will be initially pretested with a sample of approximately 100 undergraduate students from the southern campus. Pretest results should indicate whether some of the scenarios may need modifications or even deletion.

According to the earlier review of empirical studies on business ethics (see Table 3), the most important background factors that may influence attributions for unethical behavior and/or judgments of ethicality are organizational culture and climate, demographic variables 
(especially age), and level of cognitive moral development. Due to limitations on survey length, this study's does not attempt to directly control (by measuring) the effects of organizational culture or climate. It is possible, however, that these differences will be randomly canceled out, considering the expected variety of organizations to be represented in the final sample.

Since empirical research evidence on moral reasoning has shown that most managers, as well as most business students, fall at Kohlberg's conventional cognitive development stages three and four, with slight variations according to the size of the firm (cf. Weber, 1990; Weber \& Green, 1991), this study will not measure managers' levels of cognitive moral development.

Personal data (respondent's age, sex, organizational position and tenure, years of work experience and educational level) will be collected at the end of the survey to allow group comparisons along key demographic information.

\section{Statistical Analysis}

The main purpose of this research investigation is to test the two theoretical models suggested by hypothesis one. These models examine the relationship between individualist or collectivist attitudes and the patterns of attribution responses. Structural equation 
modeling (with the LISREL program) is the statistical technique selected for data analysis, because it allows the effects of measurement error (on both independent and dependent variables) to be measured and partialled out from the hypothesized relationships.

The use of multiple scenarios provides an opportunity to test these models across different situations. This replication strategy can provide valuable information concerning when the theoretical models apply and when they do not.

The test of hypothesis two can be performed with multiple sample analysis, which allows LISREL models to be compared across the Hispanic and Anglo groups. Although multiple group LISREL uses the entire sample to estimate model parameters, it gives information about model fit for each group separately, and also allows specification of different parameter estimates.

A full LISREL model includes two components: a measurement model, and a latentvariable or structural model (Bollen, 1989). The former establishes the relationship between constructs and their indicators (questionnaire items), while the latter depicts only the relationship between latent variables. In this research, the independent or exogenous variables are the two factors that best describe individualism (e.g. competition and hedonism) and collectivism (e.g. family integrity and interdependence). Each set of independent variables will enter a model separately, predicting one pattern of attribution responses. The attribution 
patterns are considered the dependent or endogenous variables (i.e., explained by the model). For each scenario, there is one attribution response pattern for individualism and one attribution response pattern for collectivism (with initially three and subsequently four attribution items - see survey: question B of Section 2).

Before a full model can be specified, a multiple group confirmatory factor analysis (CFA) needs to be performed separately, for both independent and dependent variables. The logic behind this two-step procedure is to allow measurement models to be adjusted first, with estimation of these measurement models prior to their simultaneous assessment with the structural models guarantees proper respecification and reestimation of the hypothesized factor structures before any constraints are imposed on the structural parameters that link different construct measures (Anderson \& Gerbing, 1988).

Since we have one measurement model for Anglos and one measurement model for Hispanics, it is possible to see if the same factor structure emerges in the two groups or if some items have different meanings across cultures (i.e., failing to load on the same factor). This is an issue of measurement equivalence, which needs to be verified initially.

Furthermore, multiple Group CFA offers an indication of the degree to which convergent and discriminant validity are obtained for the construct measures (items significantly loading on their underlying factor or construct; distinct factors exist). For each 
measurement model, the CFA method gives information concerning its overall fit, reliability and validity of indicators, and the degree of measurement error for each particular item. 


\section{CHAPTER 4}

\section{RESULTS AND DISCUSSION}

\section{Pretest data}

This study's survey was initially pretested with a sample of 87 undergraduate students from a business and society class, at location A. Data analysis was performed using 54 of these respondents, since they constituted the only reasonably large ethnic group (Hispanics). The large majority of this sample was young ( $81.6 \%$ of respondents were less than 39 years of age) and $51.7 \%$ were female. Because this particular class is a requirement for all business majors, respondents came from different functional backgrounds (marketing, accounting, finance, management and MIS).

Few important findings came from the pretest analysis. For one, the items related to the big factors of collectivism and individualism (horizontal and vertical) did not clustered together as expected, although some of the latest work of Triandis has focused on fine-tuning them (cf. Triandis, 1993). However, the small or "content" factors (competition, hedonism, and self-reliance for individualism; closeness to the family, interdependence, sociability and family integrity for collectivism) confirmed previous studies' results: confirmatory factor analysis models with 2, 3 and 4 factors showed that the original 2-2 factor structure best described individualism and collectivism. 
With respect to the scenario items, an initial exploratory factor analysis performed on all the 36 attribution items $(6 \times 6)$ showed that each set of 6 items loaded as a separate factor, thus attesting for scenario-specificity or independence. Although within-scenario analysis (factor analysis of 6 items at a time) showed that the three items for either relationshiporiented or market-oriented attribution response pattern loaded well as a separate factor, there was no basis to consider each response category as an independent dimension, because, first, three independent expert judges were not able to consistently identify them (although they reliably classified the items as either individualistic or collectivistic), and, second, items representing the same response category (e.g., emotional or calculative) did not correlate across scenarios, largely due to the specificity referred to above.

One of the main conclusions from the pretest analysis was therefore that the response categories were not independent from each other, but could only be viewed as a set (or simply as a attribution response pattern). For the final instrument, some of the items were reworded to increase clarity and two more items were added for each scenario.

Another important finding from the pretest analysis was that for two scenarios (Mater Millers and the Auto Parts Contractor), respondents either strongly disagreed or disagreed with most of the attribution items. Interestingly, these scenarios (which described a general manager approving the use of a special milling process that pollutes the environment, and a product development employee for an Auto Parts contractor deciding not to report terrible 
test results of a transaxle to the auto manufacturer) were considered the most unethical and unacceptable among the six. Although it is possible that these items were poor justifications the decision-outcomes, one could speculate that for actions that are considered strongly harmful, there can't be any acceptable justifications.

The final version of the survey kept the original INDCOL instrument (in order to preserve its integrity), plus the 4 (scenarios) $\times 8=32$ attribution response items. The last two pages of the questionnaire remained the same, assessing the personal and professional background of respondents.

\section{Final Sample}

The final sample was collected during the Spring semester of 1997. Whenever possible, the questionnaires were introduced and distributed to respondents by the researcher, in their classroom. In a few cases however, it was the professor who personally took charge of the distribution. Approximately 450 surveys were distributed among 15 classes at the Location A, and 250 among 11 classrooms at the Location B, for a total of 700 . Although the majority of students were enrolled in an MBA program, there were also students seeking a master's in Finance, in International Business, and in Science. 
The number of questionnaires returned was 372 , for a response rate of $53.1 \%$. The researcher reviewed each questionnaire, selecting only the Anglo and Hispanic students, according to their responses to the question: "What is your ethnic background?" (see survey: personal background). Some of these questionnaires were further discarded because the respondent (although choosing a White, non-Hispanic or Hispanic background) also indicated the he/she had spent the majority of his/her childhood and/or adulthood in another country (e.g., in Europe). The final sample had 139 Anglos (white, non-Hispanic Americans) and 130 Hispanics (Americans of Hispanic heritage and Latin Americans).

Since the Hispanic group seemed more heterogeneous than the Anglo, an analysis of variance was performed for four Hispanic groups (Hispanic Americans, South Americans, Cubans, and Central Americans) using all the independent and dependent variables from the survey - 63 questions on individualism and collectivism ( 31 from the INDCOL and $8 \times 4=32$ attribution items), plus the ratings on ethicality, acceptability and frequency, for a total of 75 F-tests. Although Hispanics differed significantly in years spent in U.S. (with South Americans spending the least amount - an average of 7.6 years), their responses to all of these 75 variables were not statistically different, with only two exceptions (attribution items for scenario 1: indd 1 and cold 1 ). Further t-tests were also performed for Hispanic Americans versus all other Hispanics and for Hispanic Americans versus South Americans only. In both cases, results confirmed the initial finding, thus supporting the idea of combining all Hispanic respondents as a single homogeneous group. 
The final sample description is summarized in Tables 7 and 8 , which review the personal and professional backgrounds of Anglos, Hispanics and the total sample. Starting with the entire sample, the large majority of respondents is young, with $88 \%$ of them under or up to 39 years of age. There is a slight majority of males $(57.7 \%)$ but, as expected, the educational level for all respondents is mostly the same - a complete bachelor's degree coupled with a few graduate classes $(21.6+53.5+12.6=87.7 \%)$. A large number of respondents are Americans (71.4\%), and even a higher percentage of them have spent the majority of their childhood $(78.1 \%)$ and adulthood $(88.8 \%)$ in the United States.

Chi-square tests of independence for Anglos and Hispanics showed that there are basically two significant differences across these two groups, in terms of their personal background: Anglos are older ( $71.5 \%$ of Hispanics are under 30 and $96.9 \%$ are up to 39 years

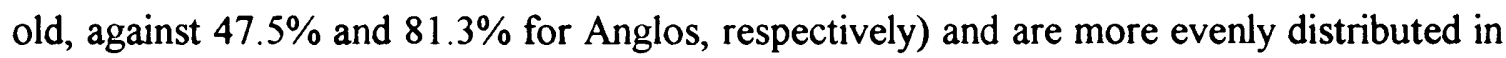
terms of gender (48.1\% males and 51.9\% females for Anglos, against $68.4 \%$ Hispanic males and $31.6 \%$ Hispanic females). In spite of the fact that $59.2 \%$ of Hispanics were born outside of U.S., the majority of them were socialized in the American culture quite early ( $56.2 \%$ were in United States for the majority of their childhood and $76.9 \%$ for the majority of their adulthood).

Professional data also suggests that most of the respondents were currently working (85.9\%), especially in the service sector (Table 8). In addition, slightly more than half of 
these were holding positions in which they were formally supervising employees (52.2\%), with salaries ranging from $\$ 25,000$ to 50,000 per year ( $57.6 \%$ of the total sample). Also, most companies that they work for have adopted some type of ethics code (71\%), although notably there is not too much emphasis in enforcement.

The only difference between Hispanics and Anglos (in terms of professional background) is the extent to which their companies have adopted ethics codes: more Hispanics worked for companies that did not have such a code (35.5\% versus $23.1 \%)$, which could be an indication that they work for smaller or more entrepreneurial firms, or that managers in these companies don't think that ethics codes are relevant.

Further comparison between the analysis of variance (ANOVA) performed for the 4 Hispanic groups and a ANOVA for 5 groups (4 Hispanic groups + Anglos) showed that the age difference was mainly detected within the Hispanic group: Hispanic and Central Americans were significantly younger than the other three groups. Since the variable age did not affect Hispanic responses on the independent and dependent variables (as explained above), it is reasonable to conclude that there is no need to control for its effects.

I did, however, compare Hispanic males versus Hispanic females and Anglo males versus Anglo females to see if gender would affect responses on the variables of interest (31 INDCOL items +32 scenario items $=63$ F-tests). Again, analysis of variance results showed 
only one statistically significant difference between Hispanic males and females, and only three between Anglo males and females. So one could safely conclude that gender is not an important factor in explaining INDCOL and scenario responses in this study.

Overall, it is noteworthy that even the small significant differences in the background of these graduate students did not affect their responses to the independent and dependent variables. Although t-tests comparing the two groups (Anglos vs. Hispanics) along the key continuous variables ( $31 \mathrm{INDCOL}$ items plus 32 scenario items $=63 \mathrm{t}$-tests) showed that there are a few important differences among them (i.e., two closeness to the family items, two hedonism items, two competition items, one sociability item and two scenario items; or 9 statistically significant differences out of $63 \mathrm{t}$-tests $=14 \%$, which is more than one would expect by change alone), these two groups are fairly similar with respect to their average responses across the variables of interest. This finding can be attributed to the fact that the sample was composed of business students only. If we had sampled Nursing or Arts students as well, probably more significant differences would have emerged. 


\section{Data Analysis}

Confirmatory Factor Analyses on Independent and Dependent Variables

The strategy used to analyze both independent and dependent variables was the following: (1) exploratory factor analysis (for Hispanics, for Anglos, and for the entire sample); (2) independent confirmatory factor analysis for each ethnic group; (3) multiple group confirmatory factor analysis with all parameters constrained to be equal across groups (model A); and (4) hypothesis testing for alternative models (through a chi-square difference test, model $\mathrm{A}$ is compared to alternative models which release 1 parameter constraint at a time).

Independent Variables (attitude items from INDCOL):

Although Triandis has successfully replicated a four (2 and 2) factor structure for individualism and collectivism a number of times, he has suggested that the contents of these constructs should be allowed to vary from culture to culture. His usual approach has been therefore to factor analyze the 31 attitude items of the INDCOL (which measure competition, self-reliance, hedonism, closeness to the family, interdependence, family integrity and sociability) and see which pair of constructs best describes individualism and collectivism for the particular sample in question. This is an interesting way of combining emic and etic 
approaches. As mentioned in chapter 2, family integrity and interdependence are the factors most commonly found for collectivism, while for individualism competition usually appears, sometimes with either self-reliance or hedonism, but most frequently with separation from ingroups (the reverse of family integrity and/or closeness to the family items)

By looking at the results of the three exploratory factor analyses (unforced factors, varimax rotation) - for Hispanics, Anglos and the entire sample - it became clear that hedonism (first factor for Anglos and total sample, third factor for Hispanics), and competition (second factor for Anglos, fourth for Hispanics and fifth for the total sample) were the constructs that best described individualism, while family integrity (third factor for Anglos, second for Hispanics and the total sample) and interdependence ( fourth for Anglos, first for Hispanics and third for the total sample) worked best for collectivism. This last factor came out mixed with other collectivist items (sociability and closeness to the family), but was chosen as the one that best captured the idea of ingroup harmony, conveyed by the mixed factor.

The input for confirmatory factor analysis was the covariance and correlation matrices of the observed variables (item measures of competition, hedonism, interdependence and family integrity), generated by the PRELIS program. These two matrices are needed in order to use the weighed least squares estimation method, which is the recommended procedure for minimizing a particular fit function when all variables are ordinal, i.e., responses are classified 
into ordered categories and there is no specific unit of measurement) (Joreskog \& Sorbom, 1993). A specific type of correlation matrix is used (polychoric), which assumes an underlying continuous $\mathrm{z}$ distribution for the ordinal variables. The unstandardized version of this matrix forms an asymptotic covariance matrix (by definition, a covariance matrix is an unstandardized correlation matrix - it measures the extent of covariance among variables without dividing it by the product of their standard deviations).

The independent confirmatory factor analyses (for each ethnic group separately) were performed just to get an idea of how each parameter would look like in each sample. In the SIMPLIS language (LISREL for windows), multiple group analysis can only be accomplished when at least one equality constraint is already established (e.g., equal lambdas or factor loadings; or equal deltas or error variances). The multiple group (MG) confirmatory factor analysis, however, is a more powerful way of looking at the data, because it uses the total sample, while fitting a model that works well for both groups. Attempts to "clean" or improve the models were therefore performed at the multiple group level. Thus the following discussion of results will focus on them.

The initial MG confirmatory factor analysis model for individualism had competition and hedonism as latent variables, with their items (COM1-COM5R and HED1-HED5) loading in each construct separately. This fully constrained model (model A - all parameters are equal across groups) had a poor fit, especially for Hispanics (suggested cross-loadings of 
items). However, after dropping an item with poor reliability (which in LISREL analysis is assessed by the size of the r-square for the lambda coefficient), COM5R, and an item that was redundant (HED5, which was highly correlated with HED4), the model achieved a very good fit, with chi-square of $80.08(\mathrm{df}=54, \mathrm{p}=0.01)$ and cfi (comparative fit index, which compares the hypothesized model against an independence or baseline model, wherein no relationships are specified among the variables) of 0.93 (see Table 9). Table 10 shows all model parameters, which were significant. The phi (factor correlation) for competition and hedonism was -.20 .

Also, the two reversed items for hedonism (HED2R and HED3R) shared error variance (since the items were quite similar, I allowed the errors to correlate). Additional fit indices for model $\mathrm{A}$ are also provided in Table 9 (the goodness-of-fit index and the nonnormed fit index, which use different formulas but belong to the same class of indices as the cfi, given that they also consider predicted versus baseline model). Model A was compared and preferred over three alternative models: model B (different phis across groups), model C (different lambdas across groups) and model D (different deltas across groups). Chisquares for model B and C were practically the same as for model A (and model A is more parsimonious), while chi-square for model D was actually a lot worse (i.e., higher).

Interestingly, the sign of phi was negative, against the expected. Since both samples of Hispanics and Anglos were predominantly young, one could speculate that for this 
generation (generation $\mathrm{X}$ ) the search for pleasure is seen as in conflict with the drive to compete or seek work-related achievement.

The initial MG confirmatory factor analysis model for collectivism had interdependence and family integrity as latent variables, with their respective items loading in each construct separately (INTER1-5 and FINT1R-FINT4). This time, the fully constrained model A already had a fairly good fit, prior to any modifications. Dropping FINT1R (very low reliability, non-significant lambda) however significantly improved the model. Table 11 shows the final items for each construct. Similarly to the individualism model, all the parameters were found significant and one error covariance was also included (between INTER1 and INTER3). The phi was nonetheless stronger and positive for these two factors, as expected. The final model A had a chi-square of $60.11(\mathrm{df}=54, \mathrm{p}=0.56)$ and a cfi of 0.99 (Table 9). Again, models B and C did not improve the model with all equality constraints, while model D made the fit significantly worse.

Dependent Variables (attribution response items from scenarios 1-4):

The same four step procedure was used: 1)exploratory factor analysis; 2)independent confirmatory factor analysis; 3 ) multiple group confirmatory factor analysis with a fully constrained model; and 4)hypothesis testing for alternative models (models C and D). Since there was no prior research using these items, exploratory factor analyses were performed for 
both unforced and forced 2-factor solution (market vs relationship-oriented response patterns). When all the items where considered together (in an unforced solution), there were some scenarios for which items got clustered into one factor, while others got split into two. However, when each set of scenario items (inda- $d$ = market-oriented; cola- $d$ = relationshiporiented attributions) was factor-analyzed independently, the unforced solution systematically provided a two-factor structure for these items. The discussion that follows will summarize results for each scenario separately.

Across the three samples (Anglos, Hispanics and total) the exploratory factor analysis (forced 2 factors, varimax rotation) for scenario 1 , the bribery situation, showed that all the relationship-oriented (RO items) loaded together, with either one or two of the marketoriented (MO) items. The second factor had always items indal and indcl, with indbl sometimes loading on the first and sometimes on the second factor.

For the confirmatory factor analysis at the multiple group level, the fully constrained model for market-orientation (model A) had a good fit, prior to any modifications. Only one addition was made to this model - an error covariance between indbl and indd 1 . The chisquare for this final model was $15.24(\mathrm{df}=11, \mathrm{p}=0.17)$ and the cfi was 0.95 (see Table 12). All parameter estimates were significant (Table 13). Although alternative hypothesis testing showed that in this particular case model C (different lambdas) significantly improved overall fit, model A was chosen to maintain consistency, considering that it was the best model for 
both RO and MO attributions in all other scenarios. In fact, for this scenario also, the multiple group model A for the relationship-oriented attributions had a perfect fit, with chisquare of $6.39(\mathrm{df}=12, \mathrm{p}=0.89)$ and cfi of $1.00($ Table 12). All significant parameters can be seen in Table 14.

In scenario 2, the conflict of interest situation at Tangy Spices, results of exploratory factor analyses across the three samples placed three out of the four items for each attribution response pattern together, although in different order (for Hispanics and the total sample, the first factor was MO, while for Anglos it was the second).

The multiple group confirmatory factor analyses for both $\mathrm{MO}$ and $\mathrm{RO}$ attribution response patterns resulted in a model $\mathrm{A}$ with perfect fit $(\mathrm{cfi}=1.00)$, with chi-squares of 11.56 $(d f=12, p=0.48)$ and $4.16(d f=12, p=0.98)$, respectively (Table 12$)$. For both models, all parameters were significant and no modifications were needed (see Tables 15 and 16). Although models $\mathrm{C}$ and $\mathrm{D}$ were tested, they could not have improved model fit any further.

For scenario 3 , the conflict of interest situation at the microcomputer firm, results from exploratory factor analysis showed that the two-factor split was less clear than in earlier scenarios. Although across the three samples the first factor always had three items from one attribution response pattern, it also contained two of the other's. The second factor was consequently a 2-1 split, equally difficult to interpret. 
At the multiple group level, confirmatory factor analysis for the market-oriented attributions generated a poor initial model, which was then modified to include only three of the four items. This final model A achieved perfect fit, with chi-square of $11.56(\mathrm{df}=6$, $\mathrm{p}=0.90$ ). However, it was not necessary to revise the four-item model A for RO attributions. Even though its p-value was significant (the best models have non-significant chi-squares), the model had a very good cfi $(0.94)$, thus indicating adequate fit. This is also corroborated by the other indices Table 12). Tables 17 and 18 show the final parameter estimates for these two attribution models.

Finally, in scenario 4, the paternalism situation, results from exploratory factor analysis were similar to the previous scenario, with the three samples showing a split of 3-2 for the first factor and 2-1 for the second. Fitting model A (MG-CFA) for MO attributions required again the dropping of one item (inda4), but the final model also ended up with a close to perfect fit (chi-square of $15.24, \mathrm{df}=6, \mathrm{p}=0.17$ and $\mathrm{cfi}=0.99$ ). For the RO attributions, the single addition was an error covariance between items colc4 and cold4. This final model A had a perfect fit $(\mathrm{cfi}=1.00)$, with chi-square of $7.50(\mathrm{df}=11, \mathrm{p}=0.76)$ - see Table 12 . Parameter estimates for both models (RO and MO) were all significant (Tables 19 and 20). Once again, models $\mathrm{C}$ and $\mathrm{D}$ did not offer any major improvements. 
Overall, it can be said that the confirmatory factor analyses for both independent and dependent variables generated models with excellent fit, although it was usually easier to fit the collectivism - relationship-oriented models than the individualism- market-oriented ones. The final measurement models were all fully constrained (all parameters are equal across the two ethnic groups), which indicates that there is complete measurement equivalence for Hispanics and Anglos.

\section{Full Models}

Table 21 summarizes the resulting fit indices and r-squares for the full models. The measurement models were kept invariant, according to findings from the previous section. The strategy was therefore to run two types of multiple group models: one model with all the parameters constrained to be equal across groups, including gammas (the coefficients that estimate the relationship among constructs) versus a model in which gammas were allowed to differ for Hispanics and Anglos (model A versus model E). Even though in a couple of cases the chi-square difference test did not show major differences for these two alternatives, model E was consistently the best model for both collectivism - relationship-oriented attributions and individualism - market-oriented attributions, across all scenarios. The discussion that follows will present final results for model E, for each scenario separately. 
In scenario 1 , the bribery situation, the model for individualism-MO attributions showed competition and hedonism having a significant effect on the dependent variable MO (symbol: ind1) for Anglos, while for Hispanics only competition had a significant effect (see Tables 22 and 23). With the exception of phi (factor correlation for the two independent variables), all other models parameters were found significant for both groups. The collectivism - RO attributions model, showed interdependence having a significant effect on the dependent variable RO (coll) for both groups, while the family integrity-coll path was only significant for Hispanics (Tables 24 and 25). Interestingly, for Hispanics both interdependence and family integrity had a significantly negative effect on attributions. All other parameters, including phi, were significant for both groups.

Chi-squares for the individualism and collectivism models were $279.72(\mathrm{df}=125$, $\mathrm{p}=0.00)$ and $236.85(\mathrm{df}=126, \mathrm{p}=0.00)$, as shown in Table 21 . Fit indices indicated very good model fit for both cases, although p-values for chi-squares were significant. For the former model, r-squares for both Anglos and Hispanics were about the same (0.10 and 0.11), showing that the constructs of competition and hedonism explained approximately $10 \%$ of the variance in the attribution responses. For the latter model, interdependence and family integrity accounted for a much larger percentage or explained variance: $26 \%$ for Anglos and $27 \%$ for Hispanics. 
In scenario 2, the conflict of interest situation at Tangy Spices, the individualism - MO attributions model showed that competition significantly predicted attribution responses for Hispanics only, while hedonism did not significantly predict these responses in either group (Tables 26 and 27). All other parameters - including phi - were significant for both groups. Although the cfi $(0.91)$ for this model indicates a very good fit (chi-square of 261.32 with $\mathrm{df}=126, \mathrm{p}=0.00$ ), the $\mathrm{r}-\mathrm{squares}$ for the latent variables or structural equations showed that the amount of explained variance was low, especially for Anglos (less than 1\%, against $7.8 \%$ for Hispanics).

The model for collectivism - RO attributions was however much better, in terms of these structural relations (Tables 28 and 29). The path from interdependence to RO attributions was significant for both groups, although again with opposite signs (positive for Anglos and negative for Hispanics). As in scenario 1 (bribery), the gamma for family integrity was not significant for Anglos and significantly negative for Hispanics. The r-squares for these structural relationships were 0.22 and 0.23 for Anglos and Hispanics, respectively, thus indicating a much higher percentage of explained variance. The chi-square for this model was $250.64(\mathrm{df}=126, \mathrm{p}=0.00)$ with cfi of $0.92($ Table 21$)$. 
In scenario 3 , the conflict of interest situation at the microcomputer firm, the model for individualism - MO attributions had hedonism as the only significant predictor of attribution responses for Anglos, while competition was the significant predictor for Hispanics (Tables 30 and 31). All other parameters were significant for both groups, with the exception of phi. The chi-square for model $\mathrm{E}$ was $198.81(\mathrm{df}=105, \mathrm{p}=0.00)$ and the cfi was 0.90 , which again indicates a model with very good fit (Table 21). The r-square for Anglos was however not so high ( 0.076 or $7.6 \%$ of explained variance), although the structural relationships for Hispanics were practically two times stronger ( $\mathrm{r}$-square of 0.15$)$.

The model for collectivism - RO attributions showed the same pattern for Anglos, with the relationship between interdependence and attribution responses being positive and significant, while family integrity had not significant effect on responses. The pattern of significant and negative gammas for Hispanics was nonetheless not replicated in this scenario: interdependence did not significantly predict RO responses, while family integrity had a strongly positive effect (see Tables 32 and 33). All other parameters were significant, across the two groups.

The chi-square for this model was the highest of all cases, $288.80(\mathrm{df}=126, \mathrm{p}=0.00)$, although the $\mathrm{cfi}(0.91)$ remained relatively as high as the others (see Table 21). The r-squares however also reflect the greatest discrepancy between the structural equations for the two groups: while the independent variables (interdependence and family integrity) explain 
approximately $5 \%$ of the variance of the dependent variable for Anglos, they explain $26 \%$ of the variance for Hispanics, considering that the effect of family integrity on attributions is very high (gamma of 0.63).

In scenario 4, the paternalism situation, the model of individualism - MO attributions showed competition significantly predicting responses for both groups, although it had a surprisingly negative effect for Anglos (Tables 34 and 35). Hedonism was also a significant predictor for Hispanics. All other model parameters were significant for both groups, including phi. The chi-square for this model was 187.82 , with cfi of 0.89 . As in scenario 3 , the amount of explained variance for the structural equations model varied markedly between Anglos and Hispanics, being $8.3 \%$ for the former and $17 \%$ for the latter (as also shown in Table 21).

The model for collectivism - RO attributions had both independent variables as nonsignificant predictors for Anglos, while they positively predicted attributions for Hispanics. All other model parameter were significant for both groups (Tables 36 and 37). The chisquare for this model was $237.19(\mathrm{df}=125, \mathrm{p}=0.00)$ and the cfi was 0.92 , thus indicating a very good fit. The r-squares for the structural equations were again very different for both groups: 0.02 for Anglos and 0.14 for Hispanics. 
Overall, it is noteworthy that the collectivism - RO attributions models were usually better than the ones for individualism - MO attributions, in terms of structural relationships (r-squares ranged from 0.02 to 0.27 and from 0.005 to 0.17 , respectively). Also, both models were always better for Hispanics, although in the first two scenarios, the discrepancy between r-squares was rather small. However, it jumps to practically twice as much for the individualism - MO models in scenarios 3 and 4, and is even higher than double for the collectivism - RO models). For scenarios 3 and 4, the postulated relationships between individualism and collectivism on one side, and attribution responses on the other, are much less important for Anglos than for Hispanics.

Since these scenarios were the last two sets of questions in the survey (before the background information), one could speculate whether the Anglo group felt more uncomfortable with the questionnaire's length and therefore partially lost interest or attention. Although there were no significant differences in ratings of ethicality and acceptability across the two ethnic groups (for all scenarios), Anglos did think that the unethical situations described by the last three scenarios occurred less frequently, as compared to Hispanics (this mean difference was however not statistically significant). It is thus possible that the situations described by scenarios 3 and 4 are simply less meaningful for Anglos (i.e., they couldn't really relate to them). 
One explanation for the generally lower performance of the individualism - MO attributions models is that its weakest construct, hedonism, only significantly predicted attribution responses twice for Anglos (in scenarios 1 and 3) and one time for Hispanics (in scenario 4), although consistently in the positive direction. On the other hand, competition significantly (and positively) predicted Hispanic responses in all cases, but failed to predict Anglo responses in a consistent way. (it was non-significant for scenarios 2 and 3, positive for scenario 1 and negative for scenario 4).

For the collectivism - RO attributions models, interdependence was the strongest predictor of scenario responses, being mostly positive for Anglos (in scenarios 1, 2 and 3; not significant in scenario 4) and mostly negative for Hispanics (in scenarios 1 and 2; not significant for scenario 3; positive for scenario 4). Family integrity did not explain Anglo responses in all cases, but it significantly did so for Hispanics across all scenarios (it had a negative effect on responses for scenarios 1 and 2 and a positive effect for scenarios 3 and 4).

Interesting patterns can be uncovered by examining the role of these four independent constructs across scenario situations. For one, the role of hedonism was found to be much less important than expected. For this relatively young sample, hedonism may not seem as something that reinforces a competitive, work-related drive, but instead as something that minimizes it. Hedonism, however, as a general conception of life based on pleasure, did seem to affect some justifications for wrong-doings. 
The role of competition and interdependence was strong and very revealing, in terms of group differences. The former played a consistent role for Hispanics in all scenarios (it had a positive effect on market-oriented attributions), while it only had a positive effect for Anglos in a situation of bribery, wherein the idea of competition itself was being precluded. In other instances, competition had either no significant effect on Anglo responses or a negative one. For the interdependence construct, it is again noteworthy that it played a similar role for Anglos in three out of four scenarios (it had a positive effect on attributions), while it was also not so consistent for Hispanics.

Since the idea of competition is more accepted by Anglos, whereas interdependence is more accepted by Hispanics, it is reasonable to find that these cultural ideas play a more varied role for them. Also, and because these attitudes may conflict with deeper values or cultural principles held by the ethnic group (cf. competition vs. personalization for Hispanics), one can speculate whether these ideas of competition and interdependence are transmitted to members of the other ethnic group in a narrow way. This explanation is partially reinforced by consistently bigger standard deviations of the competition items for Anglos (COM2COM4), although for interdependence the evidence was contrary to expectation (standard deviations were bigger for hispanics only for INTER3).

Since a positive effect on negative type of attributions could be interpreted as endorsing the "negative" (i.e., the respondent finds the attributions acceptable), the mainly 
positive effect of interdependence on attributions for Anglos and of competition on attributions for hispanics may be an indication that a sort of perverse acculturation process has occurred. To be sure, the more a particular Hispanic respondent endorsed the idea of competition, the more s/he found certain types of justifications for unethical behavior acceptable, which means that s/he also would probably be more inclined to accept the behavior itself. I remind the reader that one of the crucial findings from the pretest analysis was that for actions were judged as strongly unethical and unacceptable, there were no set of justifications or attributions that were particularly endorsed.

The role of family integrity was certainly the most obscure of the four constructs. While it did not explain Anglo responses across all scenarios, it played an ambivalent role for Hispanics, sometimes having a positive effect on attributions (scenarios 3 and 4) and sometimes negative (scenarios 1 and 2). It is certain, however, that it is an important influence on Hispanics' responses, considering that it was a significant predictor in all cases.

To conclude, I summarize these findings by evaluating whether they support or disconfirm the main hypotheses for this study. Hypothesis one suggested that individualist and collectivist attitudes (as measured by the constructs of competition, hedonism, interdependence and family integrity) would predict a market-oriented and a relationshiporiented pattern of attribution responses, respectively. Results from multiple group LISREL analyses performed for four different scenarios overall confirmed the hypothesized 
relationships for the two ethnic groups, although in different degrees. Although all the full models showed very good fit, it became evident that the four independent constructs had shifting roles, according to the ethnic group, as well as the nuances of the situation (which can account for changes in the direction of coefficients for each ethnic group).

Hypothesis two suggested that the strength of the relationship of individualist attitudes - MO attributions would be stronger for Anglos vis-a-vis Hispanics, while the relationship of collectivist attitudes - RO attributions would be stronger for Anglos. This hypothesis was only partially supported, with family integrity being a stronger predictor for Hispanics in all scenarios, and hedonism being a little better for Anglos in three of the four vignettes. However, findings for competition and interdependence were the opposite of what was expected: the predictions were strongest for Hispanics in the case of competition and for Anglos in the case of interdependence. Overall, however, there is enough evidence to support the idea that ethnic diversity moderates these theoretical relationships.

\section{Discussion}

It is interesting to look at the current research findings in light of the growing interest or need to understand cross-cultural influences in work behavior. The following paragraphs elaborate the most important results from this study, in terms of their relevance to the question: when and how culture makes a difference? 
One of the main findings from this research was that Anglos and Hispanics viewed constructs in a similar way. The fact that measurement models for both independent and dependent variables were perfectly equivalent for Anglos and Hispanics means that there are no differences in the way these two groups define such key ideas as competition, family integrity, and attributions for wrong-doings. This finding is further reinforced by the lack of significant differences in the extent to which they endorsed these ideas, given that comparative tests of mean differences across the two ethnic groups showed only minor differences in certain items. Therefore, not only there is a good amount of similarity in the way Anglos and Hispanics understand certain key concepts, but also in terms of how much they value them.

Although one could speculate that these findings are an artifact of the sample chosen for this study (homogeneity could have resulted from the fact that all respondents were business graduate students), the issue of convergence - divergence (of values, attitudes, and behaviors) has been extensively debated in the international business literature, as discussed before (Ralston et al., 1993). This study contributes to this debate by suggesting where convergence can be found, i.e., at the level of construct definitions, or construct validity.

On the other hand, results from this study also confirmed the expectation of crosscultural differences between Anglos and Hispanics. By examining the relationships between attitudes (such as hedonism and interdependence) and attributions for unethical work behavior across all four scenarios, there is enough evidence to conclude that there are significant 
differences in the way Anglo and Hispanics use these concepts in order to explain situations. Contrary to findings from the measurement models, coefficients assessing the relationship between the independent and dependent variables (as shown in the full models) were found to be consistently different across the two ethnic groups, thus indicating that the strength and direction of the predictions changed, according to the ethnic group. It is therefore at the level of relationships among constructs (or predictive validity) that differences where detected. Going back to the debate, it is perhaps only at this level that divergence can be found.

For example, in scenarios 1 (the bribery situation) and 2 (the conflict of interest situation at Tangy) the role of interdependence shifted from a positive effect for Anglos, to a negative effect for Hispanics, on relationship-oriented (RO) attributions. Similarly, in scenario 4 (the paternalism situation), the role of competition shifted from a negative effect for Anglos, to a positive effect for Hispanics, on market-oriented (MO) attributions. In terms of strength, family integrity had always a significant effect on RO attributions for Hispanics, but that effect was not significant for Anglos, in all cases. In addition, hedonism also seemed a bit more important for Anglos in explaining MO attributions.

Although Hispanics and Anglos may share a common understanding of what these attitudes and attributions mean and may even value them to a similar extent, the above findings provide enough evidence to suggest that these constructs played a different role for 
the two ethnic groups in the situations described by the scenarios. As noted in the previous chapter, the idea that competition and interdependence may conflict with deeper values for Hispanics and Anglos, respectively, may be an explanation for the following two findings: first, that competition played a consistently negative role for Hispanics (always leading to more endorsement or acceptance of unethical attributions) and second, that interdependence played a consistently negative role for Anglos. This idea may also explain why family integrity was never a significant predictor for Anglos, in all four scenarios.

Even though Hispanics and Anglos carry both individualist and collectivist cognitions, the centrality of individualism for Anglos and of collectivism for Hispanics means that there is less acceptance of certain concepts such as competition and interdependence, given the possibility of conflict with deeper values or cultural principles (cf. interdependence vs. selfreliance for Anglos; or competition vs. personalization for Hispanics).

From a managerial perspective, it is important to recognize that Anglos may need to be trained in the pros and cons of interdependence, whenever, for example, such programs that rely on teamwork are being implemented, or when joint ventures are being formed. Hispanics may also need to be trained in the pros and cons of competition, whenever, for example, reward systems are primarily based on individual achievement, or when outsourcing partners are chosen mainly on the basis of cost-benefit considerations. The difficulties that multinational companies still encounter in penetrating certain foreign markets, due to, for 
example, higher tariffs or limited quotas, could be seen as an evidence that both the local governments and society at large may still resist the idea of competition as something good, thus preferring to protect local companies, according to their paternalistic way of thinking.

Another interesting finding from this study was that hedonism as a concept did not seem to reinforce a work-oriented, competitive drive for both ethnic groups (it actually correlated negatively with the competition items), in this particularly young sample of business students. This could be an evidence that young professionals are becoming increasingly concerned with securing a balance between work and leisure, or between work and family (ref). Managers of today's organizations need to consider how greater work demands could be impacting people's aspirations for work achievement. The trade-offs between higher salaries and bigger job responsibilities vis-a-vis lower salaries and smaller responsibilities may be changing in favor of this last option.

To conclude, I go back to the question of when culture makes a difference, because findings from this study showed that cultural attitudes significantly predicted justifications for wrong-doings, but with varying degrees, according to the ethnic group, as well as the particular situation. As summarized in Table 21, r-squares for the structural equations (which link independent and dependent variables) varied from as low as $2 \%$ to as high as $27 \%$, demonstrating that the amount of explained variance attributed to culture is contingent upon the ethnic group and the type of scenario. This conclusion is consistent with Adler (1991)'s 
suggestion that the real issue is not if culture makes a difference but when and how it does. Since this research employed a particular theoretical model that postulates a link between culture and attributions for unethical behavior, it was possible to see how a set of cultural variables had shifting roles (and different predictive power), according to the ethnic group and the nuances of the situation. 


\section{CHAPTER 5}

\section{CONCLUSION}

\section{Recapitulation}

There is a growing interest in the topic of international ethics. As globalization of markets increases, there is a greater need to understand how members of different cultures conduct their businesses, what is their underlying belief system, and what are the acceptable and unacceptable practices in each country. Within the United States also, the increasingly multicultural workforce has created a continuous demand for corporate diversity programs, in order to properly assess and manage cross-cultural differences in employees' attitudes and behaviors.

Research on the topic of international ethics has not provided definitive answers concerning the role of culture in explaining differences in ethical perceptions and judgments (see below). The lack of a theoretical framework linking culture to expected outcomes is probably one of the main deficiencies in the bulk of empirical work in the field, considering that it becomes difficult to reconcile the contrasting empirical evidence. A recent look at the last three articles found on the subject (over the 1996-1997 period), confirmed the inconsistency of findings assessing the impact of culture on ethical perceptions and attitudes. While one study showed only minor differences in the attitudes towards business ethics of 
Finnish and American business students (Grunbaum, 1997), another found significant differences in the way American and New Zealand students evaluated situations involving fraud, coercion and self-interest (Okleshen \& Hoyt, 1996). Yet, the last study provided evidence of a significant degree of agreement in perceptions of ethical/unethical practices among business students from Australia, Taiwan and United States (Allmon, Chen, Pritchett, and Forrest, 1997).

The topic of ethnic diversity has also received greater attention over the last few years. A number of books have been written on the subject (cf. Cox, 1993; Morrison, 1992), and a recent special forum of the Academy of Management Review (April 1996) was devoted to the issue. However, in the academic research environment there is still confusion concerning the meaning of ethnicity. Similarly, interviews with business executives in South Florida (Kanter, 1995) attested that practitioners are not only unsure of its meaning, but are consequently unable to create policies that will turn diversity into a source of competitive advantage for their companies.

\section{Theoretical Contributions}

Two main theoretical contributions were made by this study. First, it provided a specific theoretical model which linked culture to attribution responses for unethical work behavior, thus addressing one of the main limitations of empirical research on international 
ethics. This allowed the researcher to make certain predictions concerning the impact of two dimensions of culture (individualism and collectivism) on the outcome variables.

Second, the idea of diversity was incorporated as a moderating factor. The multiple group analysis further provided an opportunity to see how the meaning and importance of a set of constructs changed, according to the ethnic group. This is a valuable and direct way of assessing what diversity really means, in practical terms (i.e., how it affects what researchers know about ethical judgements).

Since this study did not find major differences between Anglos and Hispanics in judgments of ethicality and acceptability of practices described by the scenarios (which could have helped delineate community-based norms), its contribution to Integrative Social Contracts Theory (ISCT) was mainly to attest to the role of culture in explaining why people break ethical norms. So, the cultural variable competition (or interdependence), for example, becomes an overriding principle that justifies certain types of wrong-doings.

\section{Methodological Contributions}

The development of attribution response items for Fritzsche and Becker $(1982,1983)$ 's scenarios is a unique contribution provided by this study. Although there were issues of discriminant validity between relationship-oriented and market-oriented items for each 
scenario (see below), findings from measurement models indicated accepted convergent validity for each set of attribution responses, across all four scenarios. In addition, the researcher asked nine expert judges to classify the thirty-two items as either market or relationship-oriented types. Ratings showed that there were only seven out of the thirty-two items ( $21.9 \%$ of the items) that had inter-rater reliability below $78 \%$,i.e., that were endorsed by less than six out of the nine judges. The fact that $78.1 \%$ of the items had either high or moderate reliabilities constitutes good evidence that the attribution response items have face validity.

This study has further extended a previous methodological contribution made by Mayer and Bailey (1994), by providing additional decision-outcomes for some of the ethics scenarios. The idea of asking respondents to look at justifications for potentially unethical outcomes offers a new and viable alternative to the usual approach of asking responding what would they do in the particular situations. This approach has been criticized for inducing social desirability responses (Randall \& Fernandes, 1991).

\section{Practical Contributions}

The practical implications of this study are twofold: for better enforcement of ethics codes in organizations, one should - aside from raising awareness of ethical norms through training programs - closely examine the reasons or overriding principles that provoke 
unethical behavior. First of all, what are the values being promoted inside of this organization? Is the competitive environment imposing too much pressure on performance? how is ingroup harmony being sustained? Who is getting rewarded in the organization (and on what basis)?

Second, from the point of view of "leveraging" diversity, business leaders should measure -- "assess rather than assume" -- the effect of current organizational policies on employee's satisfaction, commitment and performance. As in the case of ethics, a better use of diversity implies a deep understanding of how it relates to micro (e.g., leader-member interactions and group dynamics) as well as macro processes (e.g., organizational culture).

As noted in Chapter 5, there is considerable amount of convergence in the way Hispanics and Anglos define key concepts that are relevant for ethical decision-making, such as competition, interdependence and attributions for wrong-doings. Furthermore, judgments of ethicality and acceptability were not significantly different across these two ethnic groups, in most cases. From a managerial perspective, these findings mean that there is no need to develop special methods or designs for ethics enforcement in order to deal with a culturally diverse workforce.

However, and to the extent that differences across ethnic groups remain in the way concepts are used or combined to explain situations, ethics training programs should focus 
on examining the impact or role of key cultural variables in ethical judgments, as well as on the potentially different appraisal of critical events which may have important moral consequences for each particular company.

\section{Study's Limitations}

The current research findings are limited by the lack of discriminant validity of the dependent variables. Although the newly created attribution responses showed high convergent validity (as evidenced by the confirmatory factor analysis models), when the two types of responses were combined for each scenario in a two factor model, the factor correlations among them were very high, thus indicating that they are not clearly distinct. The author did try to run a one construct model with all the answers for each scenario, but these models ultimately did not achieve a proper level of fit. It is possible that further discarding of items with cross-loadings would improve discriminant validity, but the final LISREL runs have failed to provide solutions that preserved convergent validity as well.

Another limitation of the current study is that it did not consider explanatory variables other than culture. Although empirical evidence has shown demographic variables such as age and gender (see Table 3) as the strongest factors affecting ethical responses, one could speculate whether the inclusion of organizational culture would have added explanatory power to the model tested by this research. Further work with this model should therefore 
attempt to measure culture within the context of the particular organizations being represented.

In addition, since this research used only one sample of Anglo and Hispanic business graduate students, results cannot be generalized to other groups with the same ethnicity. Further studies are needed to see if findings from this study can be replicated to other samples. Considering that the researcher also collected a second independent sample (about 200 respondents in more than 20 local companies) it will be possible, however, to make some general inferences concerning Hispanics and Anglos living in this geographical area. Also, business students' responses can be compared to those of non-students.

\section{New Directions for Research}

A good amount of research on business ethics still attends to issues of ethics enforcement (Trevino \& Nelson, 1995). An interesting avenue for future research is therefore the understanding of social norm transgression, i.e., the reasons that make people behave unethically.

Because ISCT deals with the idea of social contract regulation (Dunfee, 1995), it is equipped to address issues of noncompliance. Although within this theoretical framework culture has been treated essentially as criteria for the delineation of community-specific ethical 
norms, findings from this study indicate that another way of looking at culture is that it establishes the criteria for noncompliance. Future ISCT studies can help identify what are the main overriding principles that lead individuals away from acceptable ethical norms.

Future research may also use this study's conceptualization and measurement of culture, and add the cultural variables of competition, family integrity and so on, to the set of existing predictors in current ethical decision-making models (cf., .Hunt \& Vitell, 1992). As suggested before, these models have not yet provided a comprehensive treatment of culture.

Considering that this research advanced a theoretical model linking the cultural dimensions of individualism and collectivism to attributions for unethical behavior, future empirical tests could also try to extend this model, by including other explanatory variables such as organizational culture, as well as individual factors such as the level of cognitive moral development.

Furthermore, research with the ethics scenarios could address one of this study's limitation by refining the attribution items for each response type or pattern (dropping, rephrasing, and creating new ones) and by using alternative structural models that consider both dependent variables (market -oriented and relationship-oriented attribution responses) simultaneously. This last step would allow their shared variance to be isolated as measurement 
error. A major reconceptualization may be needed, with all the independent and dependent variables being linked in the same model, for each scenario.

In addition, different cultural dimensions can be included, aside from individualism and collectivism. Based on different streams of research, few other constructs can be incorporated into the LISREL models, given that not all of the Triandis' constructs worked equally well. From Hofstede's work, for example, the idea of risk avoidance can be borrowed, but not necessarily measured in the same way (fewer items can be used and measured at the individual-level).

The current theoretical model can also be tested across a number of different ethnic groups such as African-Americans, Asians and Pacific Islanders. This would help clarify the moderating role of cultural diversity. Future studies can assure increased variability in the selected samples by considering students from different colleges or by sampling, within companies, respondents with different educational and/or professional backgrounds.

Finally, more research is needed in order to specify which components of the scenario situations are really affecting ethical responses, and in which way. The author attempted to 'decompose' each scenario in terms of its essential ingredients, but interestingly, researchers involved in this project (the author and her committee members) failed to agree in what was the essence of each scenario. 
For instance, the author felt that scenarios 1 and 2 (Rollfast Bicycle and Tangy Spices) looked more like each other, with the essence of the situation reflecting some type of breaking in the competitive rule (they could therefore be interpreted as more "market-oriented" scenarios). Because of that, the author thought it was logical to find that interdependence and competition were key constructs in these situations. Also, the last two scenarios (microcomputer firm and Auto Parts Contractor) were judged as more "relationship-oriented", since they bring issues of loyalty and paternalism. This would help explain the fact that the last two scenarios were less meaningful for Anglos.

However, and until an agreement can be reached concerning the specific contribution of each situation, it is not possible to speculate what kind of impact they are making on attribution responses. For example, even though the last scenario has been described by its original authors as introducing an issue of paternalism, it was not easily interpreted as such by all the researchers involved in this project.

Further empirical research is therefore needed to help delineate the specific situational components that make each scenario unique, which in turn would lead to a better understanding of the particular contextual influences on ethical judgments. 
REFERENCES 


\section{REFERENCES}

Abratt, R, Nel, D., \& Higgs, N.S. 1992. An Examination of the Ethical Beliefs of Managers Using Selected Scenarios in a Cross-Cultural Environment. Journal of Business Ethics, 11:29-35.

Adler, N.J. 1991. International Dimensions of Organizational Behavior. Massachusetts: PWS-KENT Publishing Company.

Adler, N.J., \& Ghadar, F. 1990. International Strategy from the Perspective of People and Culture: The North American Context. In A.M.Rugman (Ed.), Research in Global Strategic Management: International Business Research for the Twenty-First Century; Canada's New Research Agenda, vol 1:179-205.

Akaah, I.P. 1989a. Organizational Culture and Ethical Research Behavior. Journal of the Academy of Marketing Science, 21(1):59-63.

Akaah, I.P. 1989b. Differences in Research Ethics Judgments Between Male and Female Marketing Professionals. Journal of Business Ethics, 8:375-381.

Alexander, C.S., \& Becker, H.J. 1978. The use of vignettes in survey research. Public Opinion Quarterly, 42(1):93-104.

Allmon, D.E., Chen, H.C.K., Pritchett, T.K., \& Forrest, P.J. 1997. A Multicultural Examination of Business Ethics Perceptions. Journal of Business Ethics, 16:183188.

Amba-Rao, S.C. 1993. Multinational Corporate Social Responsibility, Ethics, Interactions and Third World Governments: An Agenda for 1990s. Journal of Business Ethics, 12:553-572.

Anderson, J C., \& Gerbing, D.W. 1988. Structural Equation Modeling in Practice: A Review and Recommended Two-Step Approach. Psychological Bulletin, 103(3):411-423.

Aram, J.D. 1989. The Paradox of Interdependent Relations in the Field of Social Issues in Management. Academy of Management Review, 14(2):266-283.

Armstrong, R.W. 1992. An Empirical Investigation of International Marketing Ethics: Problems Encountered by Australian Firms. Journal of Business Ethics, 11:161-171. 
Aristotle, 1962. Nicomachean Ethics, Bk II, Translation by M. Ostwald. New York: The Bobbs-Merril Company.

Arlow, P. 1991. Personal Characteristics in College Student's Evaluations of Business Ethics and Corporate Social Responsibility. Journal of Business Ethics, 10:63-69.

Atsumi, R. 1980. Patterns of Personal Relationships: A Key to Understanding Japanese Thought and Behavior. Social Analysis, 6:63-78.

Bagozzi, R.P., \& Phillips, L.W. 1991. Assessing Construct Validity in Organizational Research. Administrative Science Quarterly, 36:421-458.

Bagozzi, R.P., \& Phillips, L.W. 1982. Representing and testing organizational theories: A holistic construal. Administrative Science Quarterly, 27:459-489.

Barlund, D.C. 1975. Public and Private Self in Japan and the United States. Tokyo: Simul Press.

Baron, R.M., \& Kenny, D.A. 1986. The Moderator-Mediator Variable Distinction in Social Psychological Research: Conceptual, Strategic, and Statistical Considerations. Journal of Personality and Social Psychology, 51(6):1173-1182.

Baumhart, S.J. 1961. How Ethical are Businessmen? Harvard Business Review, 39(4):631.

Beauchamp, T.L., \& Bowie, N.E. 1983. Ethical Theory and Business. Englewood Cliffs, NJ: Prentice Hall.

Becker, H., \& Fritzsche, D.J. 1987. A comparison of the Ethical Behavior of American, French and Grman Managers. The Columbia Journal of World Business, 22(4):8797.

Bell, D. 1987. The World and the United States in 2013. Daedalus, 116:1-31.

Benedict, R. 1934. Anthropology and the Abnormal. The Journal of General Psychology, 10:59-82.

Berger, P.L., \& Luckmann, T. 1966. The Social Construction of Reality. New York: Doubleday \& Company. 
Berman, J.J., Murphy-Berman, V., \& Singh, P. 1985. Cross-Cultural Similarities and Differences in Perceptions of Fairness. Journal of Cross-Cultural Psychology, 16:55-67.

. Betz, M., O'Connell, L., \& Shepard, J.M. 1989. Gender Differences in Proclivity for Unethical Behavior. Journal of Business Ethics, 8:321-324.

Blasi, A. 1980. Bridging Moral Cognition and Moral Action: A Critical Review of the Literature. Psychological Bulletin, 88:1-45.

Boal, K.B., \& Berry, N. 1985. The cognitive structure of corporate social responsibility. Journal of Management, 11(3):71-82.

Boddewyn, J. 1969. Comparative Management \& Marketing: Text and Readings. Illinois: Scott Foresman \& Company.

Bollen, K.A. 1989. Structural Equations with Latent Variables. New York: John Wiley \& Sons.

Bollen, K.A., \& Long, J.S. 1993. Testing Structural Equation Models. Newbury Park: Sage Publications.

Bommer, M., Cratto, C., Gravander, J., \& Tuttle, M. 1987. A Behavioral Model of Ethical and Unethical Decision Making. Journal of Business Ethics, 6(4):265-280.

Bond, M.H., Chiu, C., \& Wan, K. 1984. When modesty fails: The social impact of group effacing attributions following success or failure. European Journal of Social Psychology, 16:111-127.

Bontempo, R, Lobel, S., \& Triandis, H.C., 1990. Compliance and Value Internalization in Brazil and the U.S.: Effects of Allocentrism and Anonymity. Journal of CrossCultural Psychology, 21:200-213.

Bontempo, R., \& Rivera, J.C. 1992. Cultural Variation in Cognition: The Role of SelfConcept in the Attitude-Behavior Link. Paper presented at the 52nd Annual Meeting of the Academy of Management, Las Vegas.

Boulding, K.E. 1952. Religious Foundations of Economic Progress. Harvard Business Review, 30:33-40.

Bougon, M., Weick, K.,\& Binkhorst, D. 1977. Cognition in Organizations: An Analysis of the Utrecht Jazz Orchestra. Administrative Science Quarterly, 22:606-639. 
Bornheim, G. 1993. O Sujeito e a Norma. In A.Novaes (Ed.), Etica: 247-259. Sao Paulo: Companhia das Letras.

Boyacgiller, N., \& Adler, N.J. 1991. The Parochial Dinosaur: Organizational Science in a Global Context. Academy of Management Review, 16(2):262-290.

Brabeck, M. 1984. Ethical Characteristics of Whistle Blowers. Journal of Research in Personality, 18:41-53.

Brady, F.N., \& Hatch, M.J. 1992. General Causal Models in Business Ethics: An Essay on Colliding Research Traditions. Journal of Business Ethics, 11:307-315.

Brenner, S.N., \& Molander, E.A. 1977. Is the Ethics of Business Executives Changing? Harvard Business Review, 55:57-71.

Broehl, W.G., Jr. 1958. Looking Around: Do Business and Religion Mix? Harvard Business Review, 36:139-144.

Buchholz, R.A. 1979. Business Environment/Public Policy: A Study of Teaching and Research in Schools of Business and Management. Missouri: Washington University Center for the Study of American Business.

Buller, P.F., Kohls, J.J., \& Anderson, K.S. 1991. The Challenge of Global Ethics. Journal of Business Ethics, 10:767-775.

Burnett, J.H., \& Karson, M.J. 1987. Personal Values and Business Decisions. Journal of Business Ethics, 6:371-382.

Campbell, D.T., \& Fiske, D.W. 1959. Convergent and discriminant validation by the multitrait-multimethod matrix. Psychological Bulletin, 56:81-105.

Campbell, T.C., Jr. 1957. Capitalism and Christianity. Harvard Business Review, 35:37-44.

Carroll, A.B. 1979. A three-dimensional conceptual model of corporate social performance. Academy of Management Review, 4:497-505.

Carroll, S.J., \& Gannon, M.J. 1997. Ethical Dimensions of International Management. California: Sage Publications.

Cavanagh, S.J., \& Fritzsche, D.J. 1985. Using vignettes in business ethics research. In Lee Preston (Ed.), Research in corporate social performance and policy, 7:279-293. 
Certo, S.C. 1992. Principles of Modern Management: Functions and Systems. Massachusetts: Allyn and Bacon.

Chaui, M. 1993. Publico, Privado, Despotismo. In A.Novaes (Ed.), Etica: 345-390. Sao Paulo: Companhia das Letras.

Chonko, L.B., \& Hunt, S.D. 1985. Ethics and Marketing Management: An Empirical Examination. Journal of Business Research, 13:339-359.

Churchill, L.R. 1982. The Teaching of Ethics and Moral Values in Teaching: Some Contemporary Confusion. Journal of Higher Education, 53(3):296-306.

Clinard, M.B., \& Yeager, P.C. 1980. Corporate Crime. New York: Free Press.

Cochran, P.L., \& Wood, R.A. 1984. Corporate social responsibility and financial performance. Academy of Management Journal, 27:42-56.

Conrad, M.R. 1978. Are Business Ethics Worth Studying? Business and Society Review, 27:54-57.

Cousins, S. 1989. Culture and selfhood in Japan and the U.S. Journal of Personality and Social Psychology, 56:124-131.

Cox, T. 1993. Cultural Diversity in organizations: Theory, research and practice. San Francisco:Berrett-Koehler.

Dalton, D.R., \& Kesner, I.F. 1988. On the Dynamics of Corporate Size and Illegal Activity: An Empirical Assessment. Journal of Business Ethics, 7:861-870.

Davis, K. 1973. The case for and against business assumption of social responsibilities. Academy of Management Journal, 16:312-322.

DaMatta, R. 1993. Conta de Mentiroso: Sete ensaios de antropologia brasileira. Rio de Janeiro: Rocco.

DaMatta, R. 1991. Carnivals, rogues, and heroes: An Interpretation of the Brazilian dilemma. Notre Dame, IN: University of Notre Dame Press.

DaMatta, R. 1987. A Casa \& a Rua. Rio de Janeiro: Guanabara S.A.

DeGeorge, R.R. 1982. Business Ethics. New York: Macmillan Publishing. 
Delaney, J.T., \& Sockell, D. 1992. Do Company Ethics Training Programs Make a Difference? An Empirical Analysis. Journal of Business Ethics, 11:719-727.

Demos, R. 1955. Business and the Good Society. Harvard Business Review, 33:33-44.

Dill, D.D., Donaldson, T.J., Goodpaster, K.E.,\& May, W.W. 1979. Syllabi for the teaching of management ethics. A publication of the Summer Institute on Ethical Issues in the Management of Public and Private Institutions, New Haven:Connecticut.

Dion, K.L., \& Dion, K.K. 1988. Romantic love: Individual and cultural perspectives. In R.J.Stenberg \& M.L.Barnes (Eds.), The psychology of love. New Haven: Yale University Press.

Dion, K.K., Pak, A.W., \& Dion, K.L. 1990. Stereotyping Physical Attractiveness: A Sociocultural Perspective. Journal of Cross-Cultural Psychology, 21:378-398.

Doi, T. 1986. The anatomy of conformity: The individual versus society. Tokyo: Kodansha.

Doktor, R., Tung, R.L., \& Von Glinow, M.A. 1991. Future Directions for Management Theory Development. Academy of Management Review, 16(2):362-365.

Donaldson, T. 1989. The Ethics of International Business. New York: Oxford University Press.

Donaldson, T., \& Dunfee, T.W. 1994. Toward a Unified Conception of Business Ethics: Integrative Social Contracts Theory. Academy of Management Review, 19(2):252284.

Donaldson, T., \& Dunfee, T.W. 1995. Integrative Social Contracts Theory: A communitarian Conception of Economic Ethics. Economics and Philosophy, 11(1):85-112.

Dozier, J.B., \& Miceli, M.P. 1985. Potential Predictors of Whistle-Blowing: A Prosocial Behavior Perspective. Academy of Management Review, 10:823-836.

Dubinsky, A.J., Jolson, M.A., Kotabe, M. \& Un Lim, C. 1991. A cross-national investigation of industrial salespeople's ethical perceptions. Journal of International Business Studies, 22(4):651-670.

Dunfee, T.W. 1995. An Introduction to the Special Issue on Social Contracts and Business Ethics. Business Ethics Quarterly, 5(2):167-171. 
Earley, P.C. 1989. Social Loafing and collectivism: A comparison of the United States and the People's Republic of China. Administrative Science Quarterly, 34:565-581.

Epstein, E.M. 1987. The corporate social policy process: Beyond business ethics, corporate social responsibility, and corporate social responsiveness. California Management Review, 29(3): 99-114.

Evans, W.A. 1981. Management Ethics: An Intercultural Perspective. Boston: Martinus Nijhoff Medical Publishers.

Fahey, L., \& Narayanan, V.K. 1986. Macroenvironmental Analysis for strategic management. Minnesota: West.

Fleming, J.E. 1981. Public issues scanning. In L.E.Preston (Ed.), Research in corporate social performance and policy, vol 3:154-174.

Ferrell, O.C., \& Gresham, L.G. 1985. A Contingency Framework for Understanding Ethical Decision Making in Marketing. Journal of Marketing, 49(Spring):87-96.

Ferrell, O.C., Gresham, L.C., \& Fraedrich, G. 1989. A Synthesis of Ethical Decision Models for Marketing. Journal of Macromarketing, (Fall):55-63.

Ferrell, O., \& Skinner, S. 1988. Ethical Behavior and Bureaucratic Structure in Marketing Research Organization. Journal of Marketing Research, 25:103-109.

Finney, H.C., \& Leiseur, H.R. 1982. A Contingency Theory of Organizational Crime. In S.B.Bacharah (Ed.), Research in the Sociology of Organizations, vol 1:255-299. Greenwich, CT: JAI Press.

Fishbein, M., \& Ajzen, I. 1975. Belief, attitude, intention, and behavior: An Introduction to theory and research. Reading, MA: Addison-Wesley.

Fleming, J.E. 1987. A Survey and Critique of Business Ethics Research, 1986. In W.C.Frederick,\& L.E.Preston (Eds.), Research in Corporate Social Performance and Policy, vol 9:1-23. Greenwich, CT: JAI Press.

Forgas, J.P., \& Bond, M.H. 1985. Cultural Influences on the Perceptions of Interaction Episodes. Personality and Social Psychology Bulletin, 11:75-88.

Fritzsche, D.J. 1988. An Examination of Marketing Ethics: Role of the Decision Maker, Consequence of the Decision, Management Position and Sex of the Respondent. Journal of Macromarketing, 8(3):29-39. 
Fritzsche, D.J., \& Becker, H. 1984a. Linking Management Behavior to Ethical Philosophy An Empirical Investigation. Academy of Management Journal, 27(1):166-175.

Fritzsche, D.J., \& Becker, H. 1984b. Business Ethics: A Cross-Cultural Comparison of Managers' Attitudes. Paper presented at the 44th Annual Meeting of the Academy of Management, Boston.

Fritzsche, D.J., \& Becker, H. 1983a. Ethical Behavior of Marketing Managers. Journal of Business Ethics, 2:291-299.

Fritzsche, D.J., \& Becker, H. 1983b. A Comparison of the Ethical Behavior of American and German Managers. Paper presented at the 43rd Annual Meeting of the Academy of Management, Dallas.

Fritzsche, D.J., \& Becker, H. 1982. Business Ethics of Future Marketing Managers. Journal of Marketing Education, :2-7.

Gilligan, C. 1982. In a Different Voice:Psychological Theory and Women's Development. Massachussets: Harvard University Press.

Gould, J., \& Kolb, W.L. 1964. A Dictionary of the Social Sciences. Glencoe, IL: Free Press.

Gray, B., Bougon, M.G., \& Donnellon, A. 1985. Organizations as Constructions and Destructions of Meaning. Journal of Management, 11(2): 83-98.

Greenberg, J., \& Bies, R.J. 1992. Establishing the Role of Empirical Studies of Organizational Justice in Philosophical Inquiries into Business Ethics. Journal of Business Ethics, 11:433-444.

Grunbaum, L. 1997. Attitudes of Future Managers Towards Business Ethics: A Comparison of Finnish and American Business Students. Journal of Business Ethics, 16:451-463.

Gudykunst, W.B., \& Nishiba, T. 1986. The Influence of Cultural Variability on perceptions of Communication Behavior Associated With Relationship Terms. Human Communication Research, 13:147-166.

Gudykunst, W.B., Yang, S.,\& Nishiba, T. 1987a. Cultural Differences in Self-Consciousness and Self-Monitoring. Communication Research, 14:7-36. 
Gudykunst, W.B., Yoon, Y.C., \& Nishiba, T. 1987b. The Influence of IndividualismCollectivism on perceptions of communication in ingroup and outgroup relationships. Communication Monographs, 54:295-306.

Haidt, J., Koller, S.H, \& Dias, M.G. 1993. Affect, Culture, and Morality, or Is It Wrong to Eat Your Dog? Journal of Personality and Social Psychology, 65(4):613-628.

Hall, E.T. 1959. The Silent Language. New York: Doubleday \& Company.

Hamilton, V.L., \& Sanders, J. 1983. Universals in judging wrongdoing: Japanese and Americans compared. American Sociological Review, 48:199-211.

Harris, J.R. 1990. Ethical Values of Individuals at Different Levels in the Organizational Hierarchy of a Single Firm. Journal of Business Ethics, 9:741-750.

Hartley, R.F. 1993. Business Ethics: Violations of the Public Trust. New York: John Wiley \& Sons.

Hegarty, W.H., \& Sims, H.P. 1979. Organizational Philosophy, Policies, and Objectives Related to Unethical Decision Behavior: A Laboratory Experiment. Journal of Applied Psychology, 64(3):331-338.

Hegarty, W.H., \& Sims, H.P. 1978. Some Determinants of Unethical Decision Behavior: An Experiment. Journal of Applied Psychology, 63(4):451-457.

Hershberger, S.L., Lichtenstein, P., \& Knox, S. 1994. Genetic and Environmental Influences on Perceptions of Organizational Climate. Journal of Applied Psychology, 79(1):2433.

Hoffman, M.W., \& Moore, J.M. 1982. Results of a Business Ethics Curriculum Survey Conducted by the Center for Business Ethics. Journal of Business Ethics, 2:81-83.

Hofstede, G., Neuijen, B., Ohayv, D.D., Sanders, G. 1990. Measuring Organizational Cultures: A Qualitative and Quantitative Study Across Twenty Cases. Administrative Science Quarterly, 35: 286-316.

Hofstede, G. 1984. The Cultural Relativity of the Quality of Life Concept. Academy of Management Review, 9(3):389-398.

Hofstede, G. 1983. Dimensions of National Culture in Fifty Countries and Three Regions. In J.B. Deregowski, S. Dziurawiec, \& R.C.Annios (Eds.), Expiscations in CrossCultural Psychology: 335-355. Lisse, Netherlands: Swets \& Zeitlinger. 
Hofstede, G. 1980a. Culture's Consequences: International Differences in Work-related Values. Beverly Hill, CA: Sage Publications.

Hofstede, G. 1980b. Motivation, Leadership and Organizations: Do American Theories Apply Abroad? Organizational Dynamics, Summer:42-63.

Hofstede, G., \& Bond, M.H. 1988. The Confucius connection: From cultural roots to economic growth. Organizational Dynamics, 16(4):4-21.

Hsu, F.L.K. 1983. Rugged Individualism Reconsidered. Knoxville: University of Tennessee Press.

Hsu, F.L.K. 1981. American and Chinese: Passage to Differences. Honolulu: University of Hawaii Press.

Hui, C.H., \& Triandis, H.C. 1986. Individualism-Collectivism: A Study of Cross-Cultural Researchers. Journal of Cross-Cultural Psychology, 17:225-248.

Hunt, S. 1990. Commentary on an Empirical Investigation of a General Theory of Marketing Ethics. Journal of the Academy of Marketing Science, 18(2):173-177.

Hunt, S.D., Chonko, L.B. \& Wilcox, J.B. 1984. Ethical Problems of Marketing Researchers. Journal of Marketing Research, 21(3):309-324.

Hunt. S.D., \& Vasquez-Parraga, A.Z. 1993. Organizational Consequences, Marketing Ethics, and Salesforce Supervisionl. Journal of Marketing Research, XXX (February):78-90.

Hunt, S.D., \& Vitell, S. 1992. The General Theory of Marketing Ethics: A Retrospective and Revision. In J. Quelch \& C. Smith (Eds.), Ethics in Marketing. Chicago: Richard D.Irwin.

Hunt, S.D., \& Vitell, S. 1986. A General Theory of Marketing Ethics. Journal of Macromarketing, (Spring):5-16.

Iwao, S. 1988. Social Psychological Models of Man: Isn't it Time for East to Meet West? Invited Address to the International Congress of Scientific Psychology, Sydney, Australia.

Izraeli, D. 1988. Ethical Beliefs and Behaviors Among Managers: A Cross-Cultural Perspective. Journal of Business Ethics, 7:263-271. 
James, L.R., Joyce, W.F., \& Slocum, J.W., Jr. 1988. Comment: Organizations Do Not Cognize. Academy of Management Review, 13(1):129-132.

Jansen, E., \& Von Glinow, M.A. 1985. Ethical Ambivalence and Organizational Reward Systems. Academy of Management Review, 10(4):814-822.

Janssens, M., Brett, J.M., \& Smith, F.J. 1995. Confirmatory Cross-Cultural Research: Testing the Viability of a Corporate-Wide Safety Policy. Academy of Management Journal, 38(2):364-382.

Johnson, H.L. 1957. Can the Businessman Apply Christianity? Harvard Business Review, 35:68-76.

Jones, T.M. 1991. Ethical Decision Making by Individuals in Organizations: An IssueContingency Model. Academy of Management Review, 16(2):366-395.

Joreskog, K., \& Sorbom, D. 1993. LISREL 8: Structural Equation Modeling with the SIMPLIS Language. Chicago: Scientific Software International.

Kagitcibasi, C., \& Berry, J.W. 1989. Cross-Cultural Psychology: Current Research and Trends. Annual Review of Psychology, 40:493-531.

Kahn, W.A. 1990. Toward an Agenda for Business Ethics Research. Academy of Management Review, 15:311-328.

Kant, I. 1964. Foundations of the Metaphysics of Morals, Translation by H.J.Paton. New York: Harper and Row Publishers.

Kanter, R.M. 1995. World Class: Thriving Locally in the Global Economy. New York: Simon \& Schuster.

Kashima, Y., Siegel, M., Tanaka, K., \& Kashima, E.S. 1992. Do People Believe that Attitudes Cause Behaviors? Toward a Cultural Psychology of Attribution Processes. British Journal of Social Psychology, 31:111-124.

Kashima, Y., \& Triandis, H.C. 1986. The self-serving bias in attributions as a coping strategy: a cross-cultural study. Journal of Cross-Cultural Psychology, 17:83-97.

Kelley, S.E., Ferrell, O.C. \& Skinner, S.J., 1990. Ethical Behavior Among Marketing Researchers: An Assessment of Selected Demographic Characteristics. Journal of Business Ethics, 9:681-688. 
Kidwell, J., Stevens, R., \& Bechke, A. 1987. Differences in Ethical Perceptions Between Male and Female Managers: Myth or Reality. Journal of Business Ethics, 6:451457.

Kim, U. 1994. Individualism and Collectivism: Conceptual Clarification and Elaboration. In U.Kim, H.C.Triandis, C.Kagitcibasi, S.-H.Choi, \& G.Yoon (Eds.), Individualism and Collectivism: Theory, Method, and Applications: 19-40. Thousand Oaks, California: Sage Publications.

Kim, U., Triandis, H.C., Kagitcibasi, C., Choi, S.-H., \& Yoon, G. 1994. Introduction. In U.Kim, H.C.Triandis, C.Kagitcibasi, S.-H.Choi, \& G.Yoon (Eds.), Individualism and Collectivism: Theory, Method, and Applications: 19-40. Thousand Oaks, California: Sage Publications.

Kitayama, S., \& Markus, H. 1990. Culture and emotion: The role of other-focused emotions. Paper presented at the 98th Annual Convention of the American Psychological Association, Boston.

Kitayama, S., Markus, H., Tummala, P., Kurokawa, M., \& Kato, K. 1990. Culture and selfcognition. Unpublished manuscript.

Klein, K.J., Dansereau, F., \& Hall, R.J. 1994. Level Issues in Theory Development, Data Collection, and Analysis. Academy of Management Review, 19(2):195-229.

Kohlberg, L. 1984. Essays on Moral Development, Volume II: The Psychology of Moral Development. San Francisco: Harper \& Row.

Kohlberg, L. 1981. Essays on Moral Development, Volume I: The Philosophy of Moral Development. San Francisco: Harper \& Row.

Kohlberg, L. 1976. Moral Stages and Moralization: The Cognitive Developmental Approach. In T.Likona (Ed.), Moral Development and Behavior: Theory, Research and Social Issues: 31-53. New York: Halt, Rinehart \& Winston.

Kluckhohn, F., \& Strodtbeck, F.L. 1961. Variations in Value Orientations. Illinois: Row Peterson.

Laczniak, G.R., \& Inderrieden, E.J. 1986. The influence of stated organizational concern upon ethical decision-making. Journal of Business Ethics, 6:297-307. 
Lebra, T. 1984. Japanese Women: Constraint and Fulfillment. Honolulu: University of Hawaii Press.

Lebra, T.S. 1976. Japanese patterns of behavior. Honolulu, Hawaii: East-West Center.

Lee, K-H. 1981. Ethical Beliefs in Marketing Management: A Cross-Cultural Study. European Journal of Marketing, 15:58-67.

Lefcourt, H.M. 1982. Locus of control: Current trends in theory and research. New Jersey: Erlbaum.

Leung, K. 1987. Some Determinants of Reactions to Procedural Models for Conflict Resolution: A Cross-National Study. Journal of Personality and Social Psychology, 53:898-908.

Lincoln, D.J., Pressley, M.M., \& Little, T. 1982. Ethical beliefs and personal values of top level executives. Journal of Business Research, 10:457-487.

Longenecker, J.G., McKinney, J.A., \& Moore, C.W. 1989. Do Smaller Firms Have Higher Ethics? Business and Society Review, Fall:19-21.

Lukes, S. 1973. Individualism. Oxford: Basil Blackwell.

Lysonski, S., \& Gaidis, W. 1991. A Cross-Cultural Comparison of the Ethics of Business Students. Journal of Business Ethics, 10:141-150.

Magnus, B. 1979. Eternal recurrence. Nietzsche Studien VIII:362-377.

Malinowski, C.I., \& Smith, C.P. 1985. Moral Reasoning and Moral Conduct: An Investigation Prompted by Kohlberg's Theory. Journal of Personality and Social Psychology, 49:1016-1027.

Marin, G. 1985. Validez Transcultural del Principio de Equidad: El ColectivismoIndividualismo como una Variable Moderatora. Revista Interamericana de Psichologia Occupational, 4:7-20.

Martin, J. 1992. Cultures in Organizations: Three Perspectives. New York: Oxford University Press.

Marton, S. 1993. O Eterno Retorno do Mesmo: Tese Cosmologica ou Imperativo Etico? In A. Novaes (Ed.), Etica: 205-223. Sao Paulo: Companhia das Letras. 
Mathews, M.C. 1987. Codes of Ethics: Organizational Behavior and Misbehavior. In W.C.Frederick, \& L.E.Preston (Eds.), Research in Corporate Social Performance and Policy, vol 9:107-130. Greenwich, CT: JAI Press.

Mayer, K.J., \& Bailey, J. 1994. Ethical Behavior in Marketing Scenarios: Do We Perceive Men and Women Differently? Paper presented at the annual meeting of the Academy of Management, Dallas, TX.

Matsumoto, D., Kudoh, T., Scherer, K., \& Wallbott, H. 1988. Antecedents of and reactions to emotions in the United States and Japan. Journal of Cross-Cultural Psychology, 19:267-286.

Mayo, M.A., \& Marks, L.J. 1990. An Empirical Investigation of a General Theory of Marketing Ethics. Journal of the Academy of Marketing Science, 18(2):163-171.

Meyerson, D., \& Martin, J. 1987. Cultural Change: An Integration of three different views. Journal of Management Studies, 24:623-647.

Miesling, P., \& Preble, J.F. 1985. A Comparison of Five Business Philosophies. Journal of Business Ethics, 4:465-476.

Miller, J. 1994. Cultural Diversity in the Morality of Caring: Individually Oriented Versus Duty-Based Interpersonal Moral Codes. Cross-Cultural Research, 28(1):3-39.

Miller, J.G. 1984. Culture and the development of everyday social explanation. Journal of Personality and Social Psychology, 46:961-978.

Mitchell, W.J., Lewis, P.V., \& Reinsch, N.L., Jr. 1992. Bank Ethics: An Exploratory Study of Ethical Behaviors and Perceptions in Small, Local Banks. Journal of Business Ethics, 11:197-205.

Miyake, K., Campos, J., Kagan, J., \& Bradshaw, D.L. 1986. Issues in socioemotional development. In H.Stevenson, H.Azuma, \& K.Hakuta (Eds.), Child development and education in Japan:239-261. New York: Freeman.

Morrison, A. 1992. The new leaders: Guidelines on leadership diversity in America. San Francisco: Jossey-Bass.

Morse, R.M. 1988. O Espelho de Prospero: Cultura e ideias nas Americas. Sao Paulo: Companhia das Letras. 
Murphy, P.R., Smith, J.E., \& Daley, J.M. 1992. Executive Attitudes, Organizational Size and Ethical Issues: Perspectives on a Service Industry. Journal of Business Ethics, 11:11-19.

McClelland, D.C. 1961. The Achieving Society. New Jersey: Van Nostrand.

McNichols, C.W., \& Zimmerer, T.W. 1985. Situational Ethics: An Empirical Study of Differentiators of Student Attitudes. Journal of Business Ethics, 4:175-180.

McMillan, K. 1978. The U.K. and European Experience. Paper presented at the 38th Annual Meeting of the Academy of Management, San Francisco.

Nadler, A. 1986. Help Seeking as a Cultural Phenomenon: Differences between City and Kibbutz Dwellers. Journal of Personality and Social Psychology, 51:976-982.

Narroll, R. 1983. The Moral Order. Beverly Hills, CA: Sage.

Nath, R. 1986. Role of Culture in Cross-Cultural Organizational Research. In R.N.Farmer (Ed.), Advances in International Comparative Management, 14:249-267.

Negandhi, A.R. 1975. Comparative Management \& Organization Theory: A Marriage Needed. Academy of Management Journal, 18(2): 334-344.

Negandhi, A.R. 1974. Cross-Cultural Management Studies: Too Many Conclusions, Not enough Conceptualization. Management International Review, 6:59-67.

Negandhi, A.R. 1973. A Model for Analysing Organizations in Cross-Cultural Settings: A Conceptual Scheme \& Some Research Findings. In Modern Organizational Theory: Contextual, Environmental \& Socio-cultural Variables:287-311. Kent State University Press.

Nelson, J. 1994. A Social Contract for Business: Where do We Begin?". Paper presented at the Sixth Annual International Conference on Socio-Economics, Paris.

Newstrom, J.W. \& Ruch, W.A. 1975. The Ethics of Management and the Management of Ethics. MSU Business Topics, 23:29-37.

Nozick, R. 1974. Anarchy, State, and Utopia. New York: Basic Books.

Ohmann, O.A. 1957. Search for a Managerial Philosophy. Harvard Business Review, 25:41-51. 
Ohmann, O.A. 1955. 'Skyhooks' with Special Implications for Monday through Friday. Harvard Business Review, 33:33-41.

Okleshen, M., \& Hoyt, R. 1996. Cross Cultural Comparison of Ethical Perspectives and Decision Approaches of Business Students: United States of America Versus New Zealand. Journal of Business Ethics, 15:537-549.

Paul, K. 1987. Business Environment and Business Ethics in Management Thought. In K. Paul (Ed.), Business Environment and Business Ethics: The Social, Moral, and Political Dimensions of Management:1-17.

Paul, K., \& Matasar, A.B. 1993. Business and Society: The Field and the Journal. Paper presented at the annual meeting of the Academy of Management, Atlanta, GA.

Phinney, J.S. 1996. When We Talk About American Ethnic Groups, What Do We Mean? American Psychologist, September:918-927.

Posner, B.Z., \& Schmidt, W.H. 1984. Values and the American Manager: An Update. California Management Review, 26(3):202-216.

Prebe, J.F., \& Reichel, A. 1988. Attitudes Towards Business Ethics of Future Managers in US and Israel. Journal of Business Ethics, 7:941-949.

Preston, L.E. 1986. Social Issues and Public Policy in Business and Management: Retrospect and Prospect. Maryland: Center for Business and Public Policy, University of Maryland.

Preston, L.E. 1983. Teaching Materials in Business and Society. California Management Review, 25:158-173.

Quinn, R.E., \& Walsh, J.P. 1994. Understanding organizational tragedies: The case of the Hubble Space Telescope. Academy of Management Executive, 8(1):62-67.

Ralston, D.A., Gustafson, D.J., Cheung, F.M., \& Terpstra, R.H. 1993. Differences in Managerial Values: A Study of U.S., Hong Kong and PRC Managers. Journal of International Business Studies, 24(2):249-275.

Randall, D.M., \& Fernandes, M. 1991. The social desirability response bias in ethics research. Journal of Business Ethics, 10:805-817. 
Randall, D.M., \& Gibson, A.M. 1990. Methodology in business ethics research: A review and critical assessment. Journal of Business Ethics, 9:457-471.

Rawls, J. 1971. A Theory of Justice. Cambridge: Harvard University Press.

Reindenbach, R.E., \& Robin, D.P. 1990. Toward the Development of a Multinational Scale for Improving Evaluations of Business Ethics. Journal of Business Ethics, 9:639653.

Rest, J.R. 1986. Moral Development: Advances in Research and Theory. New York: Praeger Press.

Rest, J.R. 1979. Development in Judging Moral Issues. Minneapolis: University of Minnesota Press.

Ricks, D.A., Toyne, B., \& Martinez, Z. 1990. Recent Developments in International Management Research. Journal of Management, 16(2):219-253.

Riordan, C.M., \& Shore, L.M. 1997. Demographic Diversity and Employee Attitudes: An Empirical Examination of Relational Demography Within Work Units. Journal of Applied Psychology, 82(3):342-358.

Robertson, D.C., \& Ross, W.T. 1995. Decision-Making Processes on Ethical Issues: The Impact of A Social Contract Perspective. Business Ethics Quarterly, 5(2):213-240.

Robertson, D., \& Schlegelmilch, B.B. 1993. Corporate Institutionalization of Ethics in the United States and Great Britain. Journal of Business Ethics, 12:301-312.

Robey, D. 1993. Designing Organizations. Illinois: Richard Irwin.

Robey, D., \& Azevedo, A. 1994. Cultural Analysis of the Organizational Consequences of Information Technology. Accounting Management and Information Technologies.

Roland, A. 1984. The Self in India and America: Toward a Psychoanalysis of Social and Cultural Contexts. In V. Kovolis (Ed.), Designs of Selfhood:123-130. New Jersey: Associated University Press.

Ross, L.D. 1977. The intuitive psychologist and his shortcomings: Distortions in the attribution process. In L. Berkowitz (Ed.), Advances in experimental social psychology, vol 10:173-220. New York: Academic Press. 
Rotter, J.B. 1966. Generalized Expectancies for Internal versus External Control of Reinforcement. Psychological Monographs, 609:

Ruegger, D., \& King, E.W. 1992. A Study of the Effect of Age and Gender upon Student Business Ethics. Journal of Business Ethics, 11:179-186.

Schlegelmilch, B.B., \& Houston, J. 1989. Corporate Codes of Ethics in Large UK Companies: An Empirical Investigation of Use, Content and Atitudes. European Journal of Marketing, 23(6):7-24.

Schein, E.H. 1990. Organizational Culture. American Psychologist, 45(2):109-119.

Schein, E.H. 1985. Organizational Culture and leadership. San Francisco: Jossey-Bass.

Schwartz, S.H. 1990. Individualism-Collectivism: Critique and Proposed Refinements. Journal of Cross-Cultural Psychology, 21:139-157.

Schwartz, S.H., \& Bilsky, W. 1987. Toward a Theory of the Universal Content and Structure of Values: Extensions and Cross-Cultural Replications. Journal of Personality and Social Psychology, 53:550-562.

Serwinek, P.J. 1992. Demographic \& Related Differences in Ethical Views Among Small Businesses. Journal of Business Ethics, 11:555-566.

Sethi, S.P. 1986. Developing Effective International Business Strategies in a Changing Global Environment. Paper presented at the Annual Meeting of the Academy of International Business, London.

Sethi, S.P. 1977. Business Response to Social Conflict: A Comparative Analysis. Paper presented at the 37th Annual Meeting of the Academy of Management, Orlando.

Shannon, P. 1986. Hidden Within the Pages: A Study of Social Perspective in Young Children's Favorite Books. Reading Teacher, 39:656-663.

Shweder, R.A., \& Bourne, E.J. 1984. Does the concept of the person vary cross-culturally? In R.A.Shweder \& R.A.LeVine (Eds.), Culture theory: Essays on mind, self, and emotion: 158-199. Cambridge, England: Cambridge Press University.

Shikanai, K. 1978. Effects of Self-esteem on attribution of success-failure. Japanese Journal of Experimental Social Psychology, 18:47-55. 
Singer, A. 1993. Can Ethics Be Taught? Perspectives, Challenges, and Approaches at Harvard Business School. Academy of Management Executive, 7(4):86-87.

Singhapakdi, A., \& Vitell, S.J. 1993. Personal Values Underlying the Moral Philosophies of Marketing Professionals. Business and Professional Ethics Journal, 12(1):91103.

Singhapakdi, A., \& Vitell, S.J. 1991. Research Note: Selected Factors Influencing Marketers' Deontological Norms. Journal of the Academy of Marketing Science, 19(1):37-42.

Singhapakdi, A., \& Vitell, S.J. 1990. Marketing Ethics: Factors Influencing Perceptions of Ethical Problems and Alternatives. Journal of Macromarketing, (Spring):4-18.

Small, M.W. 1992. Attitudes Towards Business Ethics Held by Western Australian Students: A Comparative Study. Journal of Business Ethics, 11:745-752.

Smircich, L. 1983. Concepts of Culture and Organizational Analysis. Administrative Science Quarterly, 28:339-358.

Socrates. 1956. The Apology, Translation by W.H.D. Rouse. In E. Warmington, \& P.G. Rouse (Eds.), The Great Dialogues of Plato. New York, NY: New American Library.

Steidlmeier, P. 1992. People and Profits: The Ethics of Capitalism. Englewood Cliffs, NJ: Prentice Hall.

Stein, B.A., \& Kanter, R.M. 1993. Why good people do bad things: A Retrospective on the Hubble fiasco. Academy of Management Executive, 7(4):58-62.

Stipek, D., Weiner, B., \& Li, K. 1989. Testing some attribution-emotion relations in the People's Republic of China. Journal of Personality and Social Psychology, 56:109116

Strong, K.C., \& Meyer, G.B. 1992. An Integrative Descriptive Model of Ethical Decision Making. Journal of Business Ethics, 11:89-94.

Swinyard, A.J., Rinne, H., \& Keng Kau, A. 1990. The Morality of Software Piracy: A Cross-Cultural Analysis. Journal of Business Ethics, 9:655-664.

Takata, T. 1987. Self-depreciative tendencies in self-evaluation through social comparison. Japanese Journal of Experimental Social Psychology, 27:27-36. 
Tanaka, Y. 1978. The analysis of subjective political culture. Gakushin Review of Law and Politics, 13:1-93.

Ting-Toomey, S. 1988. Intercultural conflict styles: A face-negotiation theory. Paper presented at the meetings of the International Communications Association, New Orleans, LA.

Trevino, L.K. 1992. Moral Reasoning and Business Ethics: Implications for Research, Education, and Management. Journal of Business Ethics, 11:445-459.

Trevino, L.K. 1986. Ethical Decison Making in Organizations: A Person-Situation Interactionist Model. Academy of Management Review, 11(3):601-617

Trevino, L.K., \& Nelson, K.A. 1995. Managing Business Ethics: Straight Talk About How to do it Right. New York: John Wiley \& Sons.

Trevino, L.K., Sutton, C.D., \& Woodman, R.W. 1985. Effects of Reinforcing Contingencies and Cognitive Moral Development on Ethical Decision Making Behavior: An Experiment. Paper present at the Annual Meeting of the Academy of Management, San Diego.

Trevino, L.K., \& Youngblood, S.A. 1990. Bad Apples in Bad Barrels: A Causal Analysis of Ethical Decision Making Behavior. Journal of Applied Psychology, 75(4):378385 .

Trafimow, D., Triandis, H.C., \& Goto, S.G. 1991. Some Tests of the Distinction Between the Private and Collective Self. Journal of Personality and Social Psychology, 60:649-655.

Triandis, H.C. 1994. Theoretical and Methodological Approaches to the Study of Collectivism and Individualism. In U. Kim, H.C. Triandis, C. Kagitcibasi, S.-H. Choi, \& G. Yoon (Eds.), Individualism and Collectivism: Theory, Method, and Applications:41-51. Thousand Oaks, California: Sage Publications.

Triandis, H.C. 1993. Collectivism and Individualism as Cultural Syndromes. Cross-Cultural Research, 27 (3\&4): 155-180.

Triandis, H.C. 1990. Cross-Cultural Studies of Individualism and Collectivism. In J.Berman (ed.), Nebraska Symposium on motivation, 1989:41-133. Lincoln: University of Nebraska Press. 
Triandis, H.C. 1989. Self and Social Behavior in Differing Cultural Contexts. Psychological Review, 96:269-289.

Triandis, H.C. 1988. Collectivism and Individualism: A Reconceptualization of a Basic Concept in Cross-Cultural Social Psychology. In G.K.Verma, \& C.Barley (Eds.), Personality, Attitudes, and Cognitions:60-95 . London: Macmillan.

Triandis, H.C. 1983. Dimensions of Cultural Variation as Parameters of Organizational Theories. Journal Of International Business Studies, : :139-169.

Triandis, H.C. 1978. Some universals of social behavior. Personality and Social Psychology Bulletin, 4:1-16.

Triandis, H.C. 1972. The Analysis of Subjective Culture. New York: Wiley.

Triandis, H.C., Bontempo, R., Betancourt, H., Bond, M., Leung, K., Brenes, A., Georgas, J., Hui, C.H., Marin, G., Setiadi, B., Sinha, J.P.B., Verma, J., Spangenberg, J., Touzard, H., \& Montmollin, G. 1986. The Measurement of Etic Aspects of Individualism and Collectivism across Cultures. Australian Journal of Psychology, 38:257-267.

Triandis, H.C., Bontempo, R., Leung, K., \& Hui, C.H. 1990. A Method for Determining Cultural, Societal, and Personal Constructs. Journal of Cross-Cultural Psychology, 21:302-318.

Triandis, H.C., Bontempo, R., Villareal, M.J., Asai, M.,\& Lucca, N. 1988. Individualism and Collectivism: Cross-Cultural Perspectives on Self-Ingroup Relationships. Journal of Personality and Social Psychology, 54:323-338.

Triandis, H.C., Leung, K., Villareal, M.V., \& Clark, F.L. 1985. Allocentric versus Idiocentric Tendencies: Convergent and Discriminant Validation. Journal of Research in Personality, 19:395-415.

Triandis, H.C., Marin, G., Lisansky, J., \& Betancourt, H. 1984. Simpatia as a Cultural Script of Hispanics. Journal of Personality and Social Psychology, 47:1363-1375.

Triandis, H.C., McCusker, C., Betancourt, H., Iwao, S., Leung, K., Salazar, J.M., Setiadi, B., Sinha, J.B., Touzard, H., \& Zaleski, Z. 1993. An Etic-Emic analysis of Individualism and Collectivism. Journal of Cross-Cultural Psychology, 24:366-383.

Triandis, H.C., McCusker, C., \& Hui, C.H. 1990. Multimethod probes of Individualism and Collectivism. Journal of Personality and Social Psychology, 59:1006-1020. 
Tsalikis, J., \& Fritzsche, D.J. 1989. Business Ethics: A Literature Review with a Focus on Marketing Ethics. Journal of Business Ethics, 8:695-743.

Tsalikis, J., \& Nwachukwu, O. 1989. Cross-Cultural Marketing Ethics: On the Ethical Beliefs Difference of Greeks and Americans. Journal of International Consumer Marketing, 1(3):45-63.

Tsalikis, J., \& Nwachukwu, O. 1988. Cross-Cultural Business Ethics: Ethical Beliefs Difference between Blacks and Whites. Journal of Business Ethics, 7:745-754.

Vance, R.J., MacCallum, R.C., Coovert, M.D., \& Hedge, J.W. 1988. Construct Validity of Multiple Job Performance Measures Using Confirmatory Factor Analysis. Journal of Applied Psychology, 73(1):74-80.

Velasquez, M.G. 1992. Business Ethics: Concepts and Cases. Englewood Cliffs, NJ: Prentice Hall.

Vernon-Wortzel, H. 1994. Business and Society: A Managerial Approach. Illinois: Richard D. Irwin.

Vitell, S.J., Davis, D.L. 1990. Ethical Beliefs of MIS Professionals: The Frequency and Opportunity for Unethical Behavior. Journal of Business Ethics, 9:63-70.

Vitell, S.J., Nwachukwu, S.L., \& Barnes, J.H. 1993. The Effects of Culture on Ethical Decision Making: An Application of Hofstede's Typology. Journal of Business Ethics, 12:753-760.

Vitell, S.J., Rallapalli, K.C., \& Singhapakdi, A. 1993. Marketing Norms: The Influence of Personal Moral Philosophies and Organization Ethical Culture. Journal of the Academy of Marketing Science, 21(4):331-337.

Vogel, D. 1982. Corporate Responsibility and the Market Ethos: A Comparison of Great Britain and the United States. Paper presented at the 42nd Annual Meeting of the Academy of Management, New York.

Wada, M. 1988. Information seeking in self-evaluation of ability [Abstract]. In Proceedings of Japanese Psychological Association Meeting, 52:222.

Walzer, M. 1992. Moral Minimalism. In W.R. Shea \& G.A.Spadafora (Eds.), The twilight of probability: Ethics and politics. Canton, MA: Science History Publications. 
Weaver, M.K., \& Ferrell, O.C. 1977. The Impact of Corporate Policy on Reported Ethical Beliefs and Behavior of Marketing Practitioners. American Marketing Association Proceedings, 41:477-481.

Weber, J. 1992. Scenarios in Business Ethics Research: Review, Critical Assessment and Recommendations. Business Ethics Quarterly, 2(2):137-160.

Weber, J. 1990. Managers' Moral Reasoning: Assessing Their Responses to Three Moral Dilemmas. Human Relations, 43(7):687-702.

Weber, J. 1981. Institutionalizing Ethics into the Corporation. MSU Business Topics, 29:47-52.

Weber, J., \& Green, S. 1991. Principled Moral Reasoning: Is it a Viable Approach to Promote Ethical Integrity? Journal of Business Ethics, 10(5):325-333.

Weiner, B., Frieze, I., Kukla, A., Reed, L., Rest, S.L., \& Rosenbaum, R.M. 1972. Perceiving the Causes of Success and Failure. Morristown, NJ: General Learning Press.

Whipple, T.W., \& Swords, D.F. 1992. Business Ethics Judgments: A Cross-Cultural Comparison. Journal of Business Ethics, 11:671-678.

Whipple, T.W., \& Wolf, D.D. 1991. Judgments of Marketing Students about Ethical Issues in Marketing Research: A Comparison to Marketing Practitioners. Journal of Marketing Education, 13:56-63.

White, L.P., \& Rhodeback, M.J. 1992. Ethical dilemmas in organization development: A cross-cultural analysis. Journal of Business Ethics, 11:663-670.

Williams, A.R., \& Coughlin, C.L.J. 1993. "In God We Trust, All Others Pay Cash:" A Prologue to Trust, Vulnerability, and Deceit in Business Organizations. Business \& Professional Ethics Journal, 12(2):67-90.

Wines, W.A.,\& Napier, N.K. 1992. Toward an Understanding of Cross-Cultural Ethics: A Tentative Model. Journal of Business Ethics, 11:831-841.

Wood, D.J. 1994. Business and Society. New York: Harper Collins.

Wood, D.J. 1991. Corporate Social Performance Revisited. Academy of Management Review, 16(4):691-718. 
Yoshida, T., Kojo, K., \& Kaku, H. 1982. A study on the development of self-presentation in children. Japanese Journal of Educational Psychology, 30:30-37.

Zabid, A.R.M.,\& Alsagoff, S.K. 1993. Perceived Ethical Values of Malaysian Managers. Journal of Business Ethics, 12:331-337.

Zey-Ferrell, M., \& Ferrell, O.C. 1982. Role-Set Configuration and Opportunity as Predictors of Unethical Behavior in Organizations. Human Relations, 35(7):587-604.

Zey-Ferrell, M., Weaver, K.M., \& Ferrell, O.C. 1979. Predicting Unethical Behavior among Marketing Practitioners. Human Relations, 32:557-561.

Zinkham, G.M., Bisesi, M., \& Saxon, M.J. 1989. MBA's Changing Attitudes Toward Marketing Dilemmas: 1981-1987. Journal of Business Ethics, 8:963-974. 
APPENDICES 


\section{APPENDIX A: SURVEY}




\section{PART I}

\section{Instructions:}

This is a personality test. There are no right or wrong answers. We want to know if you strongly agree or disagree with some statements. If you strongly agree enter a 5 in the blank space; if you strongly disagree, enter a 1 in that space. If you are unsure, enter a 3 next to the statement. If you think the question does not apply to you, use a 3 and draw a circle around the 3 .

\section{In short, use this key:}

$\begin{array}{ccccc}\begin{array}{l}\text { Strongly } \\ \text { Disagree }\end{array} & \text { Disagree } & \text { Unsure } & \text { Agree } & \begin{array}{c}\text { Strongly } \\ \text { Agree }\end{array} \\ 1 & 2 & 3 & 4 & 5\end{array}$

1. I prefer to be direct and forthright when discussing with people

2. My happiness depends very much on the happiness of those around me

3. I would do what would please my family, even if I detested that activity

4. I usually struggle through a personal problem by myself

5. I usually feel that my relationships with others are more important than my individual accomplishments

6. Winning is everything

7. It is important for me to enjoy my life

8. My choice of spouse is independent of my parents/family views

9. One should live one's life independently

10. Parents of those children who won an award have every right to feel that they themselves have earned it 
11. It is important to me that I respect decisions made by my groups (e.g. social, professional)

12. What happens to me is my own doing

13. To be superior, a person must stand alone

14. I usually sacrifice my self-interest for the benefit of my group

15. It annoys me when other people perform better than I do

16. It is important for me to maintain harmony within my group

17. It is important to me that I do my job better than others

18. I like sharing little things with my neighbors

19. I enjoy working in situations involving competition with others

20. We should keep our aging parents with us at home

21. Work must be emphasized over pleasure

22. Even if I go to a college near where my parents live, I would rather rent an apartment

23. The well-being of my co-workers is important to me

24. I enjoy being unique and different from others in many ways

25. If a relative were in financial difficulty, I would help within my means

26. Children should feel honored if their parents receive a distinguished award

27. Too much emphasis on pleasure has weakened our society 
28. If my younger brother or sister fails, I feel responsible

29. People should volunteer their services to the community even if they aren't paid

30. I often do "my own thing"

31. Competition is the law of nature

32. If a co-worker gets a prize I would feel proud

33. I am a unique individual

34. To me, pleasure is spending time with others

35. When another person does better than I do, I get tense and aroused

36. One of the most important goals of my life is for me to be happy

37. I would sacrifice an activity that I enjoy very much if my family did not approve of it

38. I like my privacy

39. Without competition it is not possible to have a good society

40. I like to live close to my good friends

41. Children should be taught to place duty before pleasure

42. Life should be fun

43. Before making a decision I like to consult widely

44. I feel good when I cooperate with others

45. I hate to disagree with others in my group 
Strongly

Disagree

1
Disagree

2
Unsure

3
Agree

4
Strongly

Agree

46. Some people emphasize winning; I am not one of them

47. Before taking a major trip, I consult with most members of my family and many friends

48. Cooperation is the law of nature

49. When I succeed, it is usually because of my abilities

50. I want my aging parents to live with me in my home 


\section{PART II}

Instructions:

The following set of scenarios introduces hypothetical work situations in which a particular decision-maker is confronted with a moral issue. At the end of each scenario, a decision is provided as an outcome for the specific situation.

Following scenario descriptions, we ask you to evaluate each decision-outcome, as well as the decision-maker's reasons for choosing a particular course of action.

\section{Rollfast Bicycle Company}

Rollfast Bicycle Company has been barred from entering the market in a large Asian country by collusive efforts of the local bicycle manufacturers. Rollfast could expect to net 5 million dollars per year from sales if it could penetrate the market. Last week a businessman from the country contacted the management of Rollfast and stated that he could smooth the way for the company to sell in his country for a price of $\$ 500,000$.

Decision: Management accepted the offer.

A.How ethical is this decision?

$\begin{array}{lcccc}\begin{array}{l}\text { Strongly } \\ \text { Unethical }\end{array} & \text { Unethical } & \text { Unsure } & \text { Ethical } & \begin{array}{c}\text { Strongly } \\ \text { Ethical }\end{array} \\ 1 & 2 & 3 & 4 & \frac{1}{5}\end{array}$

Given the circumstances, how acceptable is it?

$\begin{array}{lcccc}\begin{array}{l}\text { Very } \\ \text { Unacceptable }\end{array} & \text { Unacceptable } & \text { Unsure } & \text { Acceptable } & \begin{array}{c}\text { Very } \\ \text { Acceptable }\end{array} \\ 1 & 2 & 3 & 4 & \frac{5}{5}\end{array}$

B.Why did management accept the offer?

Indicate on a scale of 1 (strongly disagree) to 5 (strongly agree) to what extent you agree with each of the following set of reasons: 
Because $\$ 500,000$ is a small price to pay for a $\$ 5$ million market.

Because they felt offended at being kept out of the market.

Management and the businessman will team up to combat the local manufacturers.

Management should have the freedom to enter any foreign market.

Management needs a friend who is familiar with this foreign market.

Since the industry is practicing unfair competition, management should do what it takes to enter the market.

Rollfast promises to fight against collusive efforts.

Rollfast will bring more competition into this industry.

C. How frequent do you think this type of decision occurs in your industry?

$\begin{array}{lcccc}\begin{array}{l}\text { Very } \\ \text { Infrequent }\end{array} & \text { Infrequent } & \text { Unsure } & \text { Frequent } & \begin{array}{c}\text { Very } \\ \text { Frequent }\end{array} \\ 1 & 2 & 3 & 4 & 5\end{array}$

\section{Tangy Spices}

Jack Brown is vice-president of marketing for Tangy Spices, a large spice manufacturer. Though still employed at Tangy, Jack recently joined in a separate business venture with Tangy's director of purchasing to import black pepper from India. Jack's new company is about to sign a five year contract with Tangy to supply their black pepper needs. The contract is set at a price above the current market price that Tangy now pays for comparable black pepper imports.

Decision: Tangy signed the contract with Jack's new company.

A.How ethical is this decision?

Strongly Unethical Unsure Ethical Strongly

Unethical

1

2

3

4

5 
Given the circumstances, how acceptable is it?

\begin{tabular}{lcccc} 
Very & Unacceptable & Unsure & Acceptable & $\begin{array}{c}\text { Very } \\
\text { Unacceptable }\end{array}$ \\
\hline 1 & 2 & 3 & 4 & $\frac{\text { Acceptable }}{5}$
\end{tabular}

B.How does Jack justify this decision for Tangy Spices?

Indicate on a scale of 1 (strongly disagree) to 5 (strongly agree) to what extent you agree with each of the following set of reasons:

Tangy can trust Jack's new company much more than any unknown supplier.

Jack's new company is providing Tangy with a reliable, long-term source of supply.

Tangy can always break the contract if it does not turn out to be a good deal.

Tangy would want to help us start this new business.

As a large company, Tangy can afford to pay extra up front to avoid future costs of finding or switching suppliers.

Tangy wouldn't want to build any negative feelings in the relationships with its vicepresident of marketing and purchasing director.

The higher price is offset by the lower indirect purchasing costs.

Tangy feels obligated to honor this contract.

C.How frequent do you think this type of decision occurs in your industry?

$\begin{array}{lcccc}\begin{array}{l}\text { Very } \\ \text { Infrequent }\end{array} & \text { Infrequent } & \text { Unsure } & \text { Frequent } & \begin{array}{c}\text { Very } \\ \text { Frequent }\end{array} \\ 1 & 2 & 3 & 4 & 5\end{array}$

\section{Microcomputer firm}

Bill Smith, a marketing analyst, has recently accepted a job with a young vigorous microcomputer manufacturer. The microcomputer manufacturers are engaged in intense competition to become the first in the market with a software package which utilizes the 
English language and thus is easily used by the average consumer. Smith's former employer is rumored to be the leader in this software development. When Smith was hired he was led to believe his selection was based upon his management potential. The morning beginning the third week on the new job, Smith received the following memo from the president:

"Please meet with me tomorrow at 8:15 for the purpose of discussing the developments your former employer has made in microcomputer software"

Decision: Bill decided to meet with the president and answer the president's questions about his former place of employment.

A.How ethical is the decision?

Strongly

Unethical

1
Unethical

2 $\underline{\text { Unsure }}$

3
Ethical

4
Strongly

Ethical

5

Given the circumstances, how acceptable is it?

Very
Unacceptable
1

Unacceptable

Unsure

Acceptable

1

2

3

4

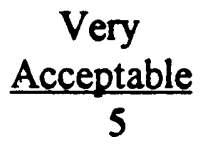

B.Why did Bill make this decision?

Indicate on a scale of 1 (strongly disagree) to 5 (strongly agree) to what extent you agree with each of the following set of reasons:

Bill wants to please his new boss.

Bill exchanges the information for job security.

Bill has the right to decide how much of his expertise he wants to disclose.

Bill now depends on his new boss.

It is more important for Bill to do the president a favor than to keep the loyalty to his old company.

The marketing analyst never signed an agreement with the old company specifying that he would not share software development. 
Bill doesn't have any close personal ties at the old company.

The new president is paying Bill and the old company is no longer paying Bill.

C.How frequent do you think this type of decision occurs in your industry?

\begin{tabular}{lcccc}
$\begin{array}{l}\text { Very } \\
\text { Infrequent }\end{array}$ & Infrequent & Unsure & Frequent & $\begin{array}{r}\text { Very } \\
\text { Frequent }\end{array}$ \\
\hline & 2 & 3 & 4 & $\frac{5}{5}$
\end{tabular}

\section{J \& P Publishing Company}

Ted Jones, senior editor of J \& P Publishing Company, has just received a manuscript from one of his most successful authors. It provides the most authoritative account yet published of the history of the development of the atomic bomb. However, the final chapter contains a detailed description of how the bomb is made. Jones has tried to convince the author to omit the last chapter stating that such information should not be made readily available to the mass market in paperback form. The author believes the chapter is critical to the success of the book and thus will not agree to its deletion.

Decision: Jones published the book including the final chapter.

A.How ethical is the decision?

$\begin{array}{lcccc}\begin{array}{l}\text { Strongly } \\ \text { Unethical }\end{array} & \text { Unethical } & \text { Unsure } & \text { Ethical } & \begin{array}{r}\text { Strongly } \\ \text { Ethical }\end{array} \\ 1 & 2 & 3 & 4 & 5\end{array}$

Given the circumstances, how acceptable is it?

\begin{tabular}{lcccc}
$\begin{array}{l}\text { Very } \\
\text { Unacceptable }\end{array}$ & Unacceptable & Unsure & Acceptable & $\begin{array}{c}\text { Very } \\
\text { Acceptable }\end{array}$ \\
\cline { 1 - 3 } & 2 & 3 & 4 & 5
\end{tabular}


B. Why did Jones publish the book?

Indicate on a scale of 1 (strongly disagree) to 5 (strongly agree) to what extent you agree with each of the following set of reasons:

Because He thinks it will be a best-seller only with that chapter.

_ Jones doesn't want to create animosity with one of his favorite authors.

It is inappropriate for a publisher to censor public information.

Jones cares most about the survival of his publishing company.

The publisher has nothing to do with how others use the information in this book.

- This one book is not really a threat to the country's security.

Publishing the chapter is an issue of freedom of speech.

Jones wants to protect the author by supporting his original idea.

C.How frequent do you think this type of decision occurs in your industry?

Very Infrequent Unsure $\quad$ Frequent

Infrequent

1

2

3

4

Frequent

5




\section{PERSONAL BACKGROUND}

Age

Less than 30

$30-39$

Gender

Male
- $40-49$

60 or older

Please indicate the highest grade in school you have completed:
High School Degree or Less

Some College Education

Bachelor's Degree
__ Some Graduate Education

Master's Degree

Ph.D
What is your ethnic background?

White, not hispanic

Hispanic

Black, not hispanic

Asian or Pacific Islander

American Indian or Alaskan Native

Were you born in United States? yes no

If not in U.S., where?

If not in U.S., how long have you been in this country? years

Where did you spend the majority of your childhood? in U.S. Outside U.S.(specify)

Where did you spend the majority of your adulthood? in U.S. Outside U.S.(specify) 


\section{PROFESSIONAL BACKGROUND}

What is your job title?

How long have you been in your current job? years

How long have you been in your current company? years

Do you formally supervise other employees? yes no

What is your functional area of expertise?

_ Management

Engineering
Production/Mfg
Marketing Other

What is your current salary?

Less than $\$ 10,000$
$\$ 25,001$ to $\$ 50,000$
$\$ 75,001$ to $\$ 100,000$

$-\$ 10,001$ to $\$ 25,000$
$-50,001$ to $\$ 75,000$
Greater than $\$ 100,000$

Please identify the one category which most accurately describes how your company derives most of its revenue:

$\begin{array}{ll}\text { Manufacturing } & \text { Services } \\ \text { Agriculture } & \text { Other(specify) }\end{array}$

Does your company have a code of ethics? yes no

Does your company have formal mechanisms for enforcing its ethics code? yes ___ no 


\section{APPENDIX B: TABLES}




\section{TABLE 1}

The Culture - Behavior Cycle *

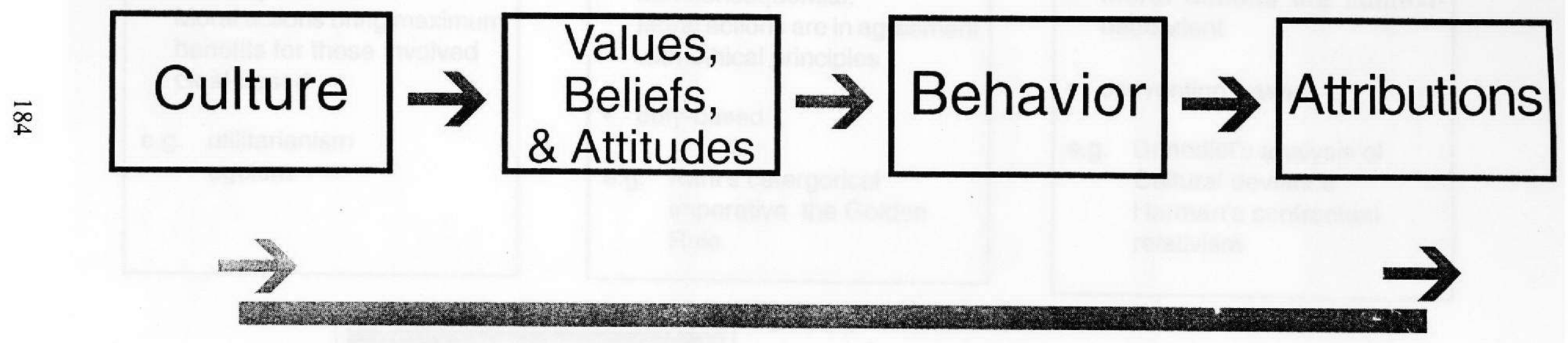

* Adapted from Adler (1991) 


\section{TABLE 2 \\ Moral Reasoning Theories}

\section{TELEOLOGICAL}

- Consequential:

Moral actions bring maximum benefits for those involved

Goal-based

e.g. utilitarianism egoism

\section{DEONTOLOGICAL}

- non-consequential:

Moral actions are in agreement with ethical principles

- duty-based

e.g. Kant's catergorical imperative the Golden Rule

\section{RELATIVISM}

- moral actions are contextdependent

- convention-based

e.g. Benedict's analysis of Cultural deviance Harman's contractual relativism

\section{MIXED}

- moral actions represent ideals to strive for

- rights and justice-based

e.g. Locke's natural rights egalitarianism libertarianism 
TABLE 3

Empirical Evidence:

Influences on Ethical Judgments, Decision-Making, Behavioral Intentions and Acceptance of Ethical Norms VARIABLES

\section{Supportive}

- type of ethical problem

- context-specificity
Fritzsche \& Becker, 1983

Fritzche. 1988

Zinckham et al., 1989

Weber, 1990

Baumhart 1961

Brenner \& Mollander, 1977
- tenure/position

- size

- org. culture / climate

- normative structure and

referent others

- ethical codes \& policies

(N) Vitell et al., 1993

(N) Singhapakdi \& Vitell, 1991

(N) Murphy et al., 1992

N) Longnecker et al.,1989

(N) Clinard \& Yeager, 1980
(+) Kelley et al., 1990

+ Longenecker et al. 1989

+ Harris, 1990

-3) Mitchell et al., 1992

(-) Dalton \& Kesner, 1988

Akaan, 1993

(+) Brenner \& Molander, 1977

+ Newstrom \& Ruch, 1975

(+) Zey-Ferrell \& Ferrel, 1982

+) Zey-Ferrell et al., 1979

+ Hunt, Chonko \& Wilcox 1984

+) Posner \& Schmidt, 1984

+ Vitell \& Davis, 1990

(+) Hegarty \& Sims, 1979

(+) Weaver \& Ferrell, 1977

+) Singhapakdi \& Vitell, 1990

(1) Trevino \& Youngblood, 1990

+) Hegarty \& Sims, 1978

+) Laczniac \& Inderrieden, 1986

\begin{tabular}{|c|c|c|}
\hline - Personal Values & & $\begin{array}{l}\text { Newstrom \& Ruch, } 1975 \\
\text { Frizsche \& Becker, } 1984 \\
\text { Hegarty \& Sims, } 1978\end{array}$ \\
\hline - CMD & & $\left\{\begin{array}{l}\text { () Trevino \& Youngblood, } 1990 \\
\text { Blasi, } 1980 \\
\text { Malinowski \& Smith, } 1985 \\
\text { Brabeck, 1984 } \\
+ \text { Trevino et al., } 1985\end{array}\right.$ \\
\hline $\begin{array}{l}\text { - Personality } \\
\text { - Mach }\end{array}$ & & $\left\{\begin{array}{l}\text { Singhapakdi \& Vitell, } 1990 \\
-3 \text { Hegarty \& Sims, 1978, } 1979\end{array}\right.$ \\
\hline$-L C$ & & $\left\{\begin{array}{l}+ \\
+\left\{\begin{array}{l}\text { Hegarty \& Sims, 1978, } 1979 \\
\text { Dozier \& Miceli, 1985 } \\
+\end{array} \text { Lefcourt, 1982 }\right.\end{array}\right.$ \\
\hline $\begin{array}{l}\text { - Demographics } \\
\text { - Age }\end{array}$ & & $\left\{\begin{array}{l}-2 \text { Mitchell et al., } 1992 \\
++ \text { Ruegger \& King, } 1992 \\
+ \text { Serwinek 1992 } \\
+ \text { Betz et al., } 1989 \\
+ \text { Burnett \& Karson, } 1987 \\
+ \text { +) Posner \& Schmidt, } 1984\end{array}\right.$ \\
\hline - Gender & 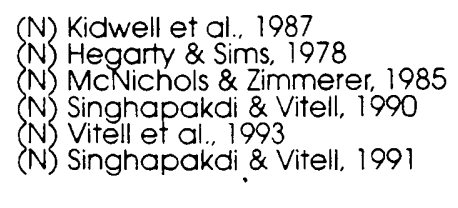 & $\begin{array}{l}\text { (F) Ruegger \& King, } 1992 \\
\text { F Arlow, } 1991 \\
\text { F, F) Fritzsche, } 1988 \\
\text { F) Akaah, } 1989 \\
\text { F) Ferrell \& Skinner, } 1988 \\
\text { (F) Gilligan, } 1982 \\
\text { (F) Chonko \& Hunt, } 1985\end{array}$ \\
\hline
\end{tabular}


TABLE 4

Cross-Cultural Studies on Business Ethics

\section{Supporting Evidence:}

AU: $\quad$ Robertson \& Schlegeimilch, 1993

IV: national culture

DV: perceived importance of ethical issues, issues of concern

SA: US and UK managers

IN: Ethics Resource Center questionnaire

F: Overall, US managers consider ethical issues more important; US companies were more concerned with employee behavior while UK firms showed greater concern for external $L$ stakeholders (customers, environment)

\section{AU: Abratt et al., 1992}

IV: national culture

DV: ethical beliefs

SA: Australian and South African marketing managers

IN: Ethical beliefs concerning employee misconduct

F: Very similar beliefs were found across the two groups; out of 28 scenarios, only three (insider trading, giving a client a bottle of whisky at Christmas, \& offering potential clients fully -paid holidays) were perceived differently in terms of the degree of ethicality (Australian managers rated them more severely)

AU: Whipple \& Swords, 1992

IV: national culture

DV: ethical judgments

SA: US and UK students

IN: Ethical issues in Marketing Research
F: US students showed a higher disapproval rating of unethical practices, as compared to UK students. Demographic differences did not affect ethical judgments, with the exception of gender (female students from both countries showed significantly higher ethical standards).

\section{AU: White \& Rhodeback, 1992}

IV: national culture

DV: perceptions of ethicality and events' likelihood of occurrence

SA: Taiwanese and US students

IN: Survey of Ethicality
F: US students provided higher ethicality ratings than the Taiwanese, with the issues of misrepresentation, value conflict, and technical ineptness being the most distinguishing between the two groups. American students also provided lower ratings on the perceived likelihood of occurrence of these unethical situations.

\section{AU: Dubinski et al., 1991}

IV: national culture

DV: perceived ethicality of hypothetical selling situations (scenarios)

SA: US, Japanese and Korean salespeople

IN: Selling ethics questionnaire
F: US salespeople perceived fewer selling situations as ethical problems, in spite of greater availability of selling policies in American companies and their stated desire to have companies address these ethical issues. 
AU: Swinyard et al., 1990

IV: national culture

DV: attitudes and behavioral intentions toward soft-

F: Students in US were more influenced by legality; stuware piracy

SA: US and Singapore students

IN: 3-item measures of knowledge concerning copydents in Singapore were less supportive of copyright laws and more influenced by outcome (benefits of software copying).

right laws, feelings related to bypassing the law and intentions toward compliance or transgression of these laws.

\section{AU: $\quad$ Becker \& Fritzsche, 1987}

IV: national culture

DV: ethical beliefs

SA: attitudes and behavior

SA: American, French \& German managers

IN: Categories of ethical problems

F: US managers were more concerned with ethical issues than their counterparts. According to the ethical problem though, there were also similarities across the three samples (e.g. conflict of interest and paternalism)

\section{Contrasting Evidence:}

\section{AU: Lee, 1981}

IV: national culture

DV: perceived ethical standards in marketing practices

SA: British and Chinese managers working in HongKong

IN: Ethics in marketing management
F: No differences of ethical standards in marketing practices were detected. Both groups subscribed to local ethical values. Middle managers held lower ethical standards than top managers. Ethical sensitivity varied according to the specific issue in question.

\section{AU: Preble \& Reichel, 1988}

IV: national culture

DV: attitude towards business ethics

SA: US and Israeli students

IN: Attitudes Towards Business Ethics Questionnaire (ATBEQ)

F: Although there were significant differences across samples, a closer examination revealed those differences were not meaningful; both groups showed relatively high moral standards.

\section{AU: Small, 1992}

IV: national culture

DV: attitudes towards ethics

SA: Western Australian students; comparable US \& Israeli students

IN: Attitudes Towards Business Ethics Questionnaire (ATBEQ)
F: Similarly to Preble \& Reichel's study, differences across three samples were not considered meaningful, reflecting rather common views amongst Western Australian, American \& Israeli students. 


\section{AU: Izraeli, 1988}

IV: national culture

DV: ethical beliefs and behavior

SA: Israeli managers; comparable sample of US managers.

IN: Ethical Beliefs and Behavior
F: In both samples, the best predictor of ethical behavior is managers' beliefs and perceptions concerning their peers' behavior.

\section{AU: Lysonski \& Gaidis, 1991}

IV: national culture

DV: ethical judgements

SA: Denmark, New Zealand \& US students

IN: Categorized ethical dilemmas (Fritzsche \& Becker, 1983)
F: Similar response pattern across samples, with a general inclination to engage in some types of unethical behavior (e. g. paying bribes, yield to pressure to award a contract to a particular supplier). Students seemed no more ethical than the practicing managers examined by Fritzsch \& Becker, 1983
AU: $\quad$ Tsalikis \& Nwachukwu, 1989
IV: national culture
DV: ethical evaluation of hypothetical dilemmas
SA: Greek and American students
IN: normative philosophy scales

F: Respondents shared similar ethical beliefs and applied similar processes to evaluate ethical situations. (factor patterns were alike)
AU: $\quad$.Tsalikis \& Nwachukwu, 1988
IV: culture
DV: ethical evaluation of hypothetical dilemmas
SA: Black and White students

F: Similar ethical beliefs were found across the two samples. In addition, evaluation of ethical scenarios was based on similar processes, according to analysis of factor patterns. 
rable 5

Bupirical Bvidence on Collectivism and Individualism

\begin{tabular}{|c|c|c|c|}
\hline $\begin{array}{l}\text { Elements of } \\
\text { Subjective } \\
\text { Culture }\end{array}$ & \multicolumn{2}{|c|}{$\begin{array}{l}\text { Defining Attributes of } \\
\text { Collectivism / Individualism }\end{array}$} & $\begin{array}{l}\text { Empirical } \\
\text { Bvidence }\end{array}$ \\
\hline $\begin{array}{l}\text { Social } \\
\text { Perception }\end{array}$ & 1. Focus: Groups & Focus: Individuals & $\begin{array}{l}\text { Triandis et al., } \\
1990 ; \text { Shannon, } \\
1986\end{array}$ \\
\hline Attributions & $\begin{array}{l}2 . \text { Other } \\
\text { behaviors: norms, } \\
\text { roles, values } \\
3 . \text { Rev. Fundamental } \\
\text { Attribution Error } \\
4 \text {.No Self-Serving } \\
\text { bias }\end{array}$ & $\begin{array}{l}\text { Other behaviors: } \\
\text { traits,attitudes } \\
\text { Fundamental } \\
\text { Attribution Error } \\
\text { Self-Serving bias }\end{array}$ & $\begin{array}{l}\text { Bontempo \& } \\
\text { Rivera, 1992; } \\
\text { Kashima et al.-, } \\
\text { 1992. } \\
\text { Miller, } 1984 \\
\text { Kashima \& } \\
\text { Triandis, } 1986 \\
\end{array}$ \\
\hline $\begin{array}{l}\text { Self- } \\
\text { Definitions }\end{array}$ & $\begin{array}{l}\text { 5.In-groups, } \\
\text { relationships } \\
6 \text {.Greater } \\
\text { knowledge of } \\
\text { others } \\
7 . \text { More Similar } \\
\text { Self } \\
8 \text {.Few Self-linked } \\
\text { memories; } \\
\text { achievement for } \\
\text { the group } \\
\text { 9.Little } \\
\text { Cognitive } \\
\text { Dissonance }\end{array}$ & $\begin{array}{l}\text { Independent entity } \\
\text { Greater knowledge } \\
\text { of self } \\
\text { Dissimilar self } \\
\text { Many Self-linked } \\
\text { memories; } \\
\text { achievement for } \\
\text { self-glory } \\
\text { Much Cognitive } \\
\text { dissonance }\end{array}$ & $\begin{array}{l}\text { Triandis et al., } \\
1990 ; \text { Trafimow et } \\
\text { al.1991 } \\
\text { Kitayama et al., } \\
1990 \\
\text { Lebra, } 1984\end{array}$ \\
\hline Goals & 10.In-group Goals & Personal Goals & Nadler, 1986 \\
\hline Emotions & $\begin{array}{l}\text { 11. Other-focused: } \\
\text { sympathy, } \\
\text { communion } \\
12 . \text { Modest people }\end{array}$ & $\begin{array}{l}\text { Ego or Self- } \\
\text { focused: anger, } \\
\text { pride } \\
\text { Self-assured people }\end{array}$ & $\begin{array}{l}\text { Markus \& } \\
\text { Kitayama, } 1991 \\
\text { Toshida et al. } \\
\text { 1982; Cousins, } \\
1989\end{array}$ \\
\hline
\end{tabular}




\begin{tabular}{|c|c|c|c|}
\hline Cognitions & $\begin{array}{l}\text { 13. What makes me } \\
\text { the same } \\
\text { 14. Needs of in- } \\
\text { group } \\
\text { 15. Context- } \\
\text { dependent }\end{array}$ & $\begin{array}{l}\text { What makes me } \\
\text { different } \\
\text { My needs, rights } \\
\text { Context-independent }\end{array}$ & $\begin{array}{l}\text { Triandis et al. } \\
1986,1988 \\
\text { Schweder \& } \\
\text { Bourne, } 1984\end{array}$ \\
\hline $\begin{array}{l}\text { Attitudes } \\
\& \text { Norms }\end{array}$ & $\begin{array}{l}\text { 16. Interdepend- } \\
\text { ence, } \\
\text { Embeddedness }\end{array}$ & Independence & $\begin{array}{l}\text { Hui \& Triandis, } \\
1986 ; \text { Triandis et } \\
\text { al. } 1986\end{array}$ \\
\hline Values & $\begin{array}{l}\text { 17. Security, } \\
\text { duty, Obedience, } \\
\text { in-group harmony, } \\
\text { hierarchy, } \\
\text { personalized } \\
\text { relationships } \\
\end{array}$ & $\begin{array}{l}\text { Pleasure, autonomy, } \\
\text { achievement, } \\
\text { freedom, } \\
\text { competition, fair } \\
\text { exchange }\end{array}$ & $\begin{array}{l}\text { Schwartz, 1994; } \\
\text { Triandis et } \\
\text { al.1985; Triandis } \\
\text { et al. 1988; } \\
\text { Gudykunst et al. } \\
\text { 1987a }\end{array}$ \\
\hline Calamity & 18.0stracism & $\begin{array}{l}\text { Dependence on } \\
\text { others }\end{array}$ & \\
\hline In-Groups & $\begin{array}{l}\text { 19. Few, but close } \\
20 . \text { Homogeneous } \\
\text { 21. Harmony } \\
\text { 22. Ascribed } \\
\text { Attributes }\end{array}$ & $\begin{array}{l}\text { Many, but casual } \\
\text { Heterogeneous } \\
\text { Confrontation } \\
\text { Achieved Attributes }\end{array}$ & $\begin{array}{l}\text { Triandis et al. } \\
1990 ; \text { Lee \& } \\
\text { Ottati, } 1990 \\
\\
\text { Triandis et al., } \\
1984 \text { a\&b; Leung, } \\
1987\end{array}$ \\
\hline $\begin{array}{l}\text { Accepted } \\
\text { Structure }\end{array}$ & 23.Hierarchical & Egalitarian & $\begin{array}{l}\text { Hofstede, } 1980 ; \\
\text { Leung \& Sedlacek, } \\
1986\end{array}$ \\
\hline $\begin{array}{l}\text { Resource } \\
\text { Allocation }\end{array}$ & $\begin{array}{l}24 . \text { Equality and } \\
\text { need }\end{array}$ & Equity & Marin, 1985 \\
\hline
\end{tabular}




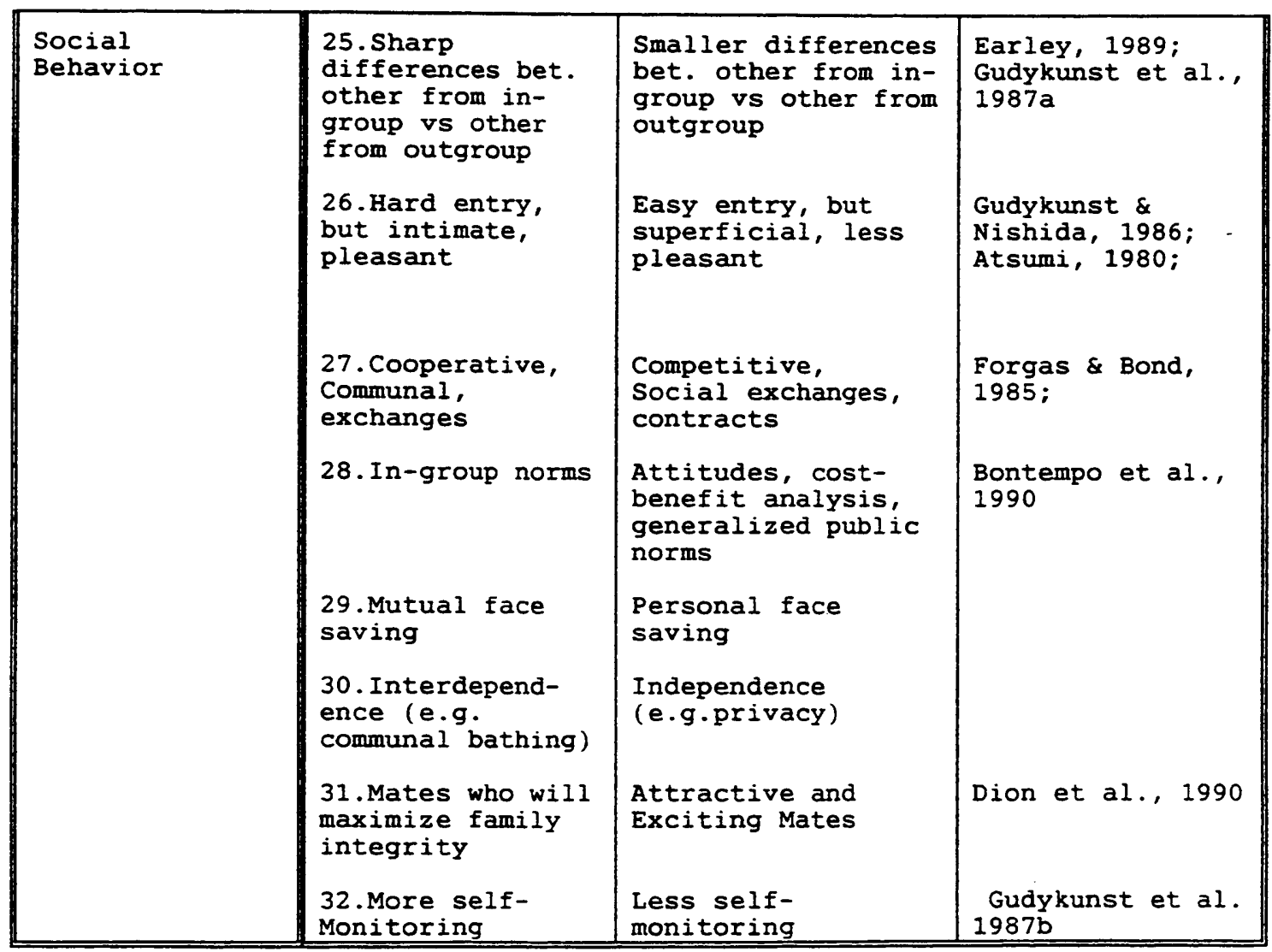


Table 6

Attribution Response Categories

For Collectivists and Individualists

\begin{tabular}{|c|l|l|}
\hline \multicolumn{1}{|c|}{ Rationale } & \multicolumn{2}{|c|}{$\begin{array}{c}\text { Attribution Response Categories } \\
\text { Collectivists: } \\
\text { Individualists: }\end{array}$} \\
\hline $\begin{array}{c}\text { Independent Self } \\
\text { Interdependent vs }\end{array}$ & $\begin{array}{l}\text { Personalized/ } \\
\text { Emotional }\end{array}$ & $\begin{array}{l}\text { Impersonal/ } \\
\text { Calculative }\end{array}$ \\
\hline $\begin{array}{l}\text { Egalitarian view } \\
\text { Egierarchical vs }\end{array}$ & $\begin{array}{l}\text { Particularistic/ } \\
\text { Driven by } \\
\text { favoritism }\end{array}$ & $\begin{array}{l}\text { General/ } \\
\text { Rules-based }\end{array}$ \\
\hline $1+2$ & $\begin{array}{l}\text { Paternalistic/ } \\
\text { Familial }\end{array}$ & Self-Reliant/ \\
\hline
\end{tabular}


Table 7

Personal Background

\begin{tabular}{|c|c|c|c|}
\hline $\begin{array}{l}\text { Variable } \\
\text { Name }\end{array}$ & $\begin{array}{l}\text { Anglos } \\
n=139\end{array}$ & $\begin{array}{l}\text { Hispanics } \\
n=130\end{array}$ & $\begin{array}{l}\text { Total Sample } \\
n=269\end{array}$ \\
\hline $\begin{array}{l}\text { Age } \\
\text { less than } 30 \\
30-39 \\
40-49 \\
50-59\end{array}$ & $\begin{array}{r}47.5 \% \\
33.8 \% \\
17.3 \% \\
1.4 \% \\
\end{array}$ & $\begin{array}{r}71.5 \% \\
25.4 \% \\
2.3 \% \\
0.8 \% \\
\end{array}$ & $\begin{array}{r}59.1 \% \\
29.7 \% \\
10.0 \% \\
1.1 \% \\
\end{array}$ \\
\hline $\begin{array}{l}\text { Gender } \\
\text { male } \\
\text { female }\end{array}$ & $\begin{array}{l}n=129 \\
48.1 \% \\
51.9 \% \\
\end{array}$ & $\begin{array}{l}n=117 \\
68.4 \% \\
31.6 \% \\
\end{array}$ & $\begin{array}{l}n=246 \\
57.7 \% \\
42.3 \% \\
\end{array}$ \\
\hline $\begin{array}{l}\quad \text { Education } \\
\text { some college } \\
\text { bachelor's } \\
\text { some grad. } \\
\text { master's } \\
\text { Ph.D } \\
\text { Executive MBA }\end{array}$ & $\begin{array}{r}- \\
18.7 \% \\
66.2 \% \\
5.0 \% \\
1.4 \% \\
8.6 \% \\
\end{array}$ & $\begin{array}{r}6.9 \% \\
24.6 \% \\
40.0 \% \\
10.8 \% \\
0.8 \% \\
16.9 \% \\
\end{array}$ & $\begin{array}{r}3.3 \% \\
21.6 \% \\
53.5 \% \\
7.8 \% \\
1.1 \% \\
12.6 \% \\
\end{array}$ \\
\hline $\begin{array}{l}\qquad \text { Origin } \\
\text { United States } \\
\text { other }\end{array}$ & $\begin{array}{c}100.0 \% \\
- \\
\end{array}$ & $\begin{array}{l}40.8 \% \\
59.2 \% \\
\end{array}$ & $\begin{array}{l}71.4 \% \\
28.6 \% \\
\end{array}$ \\
\hline $\begin{array}{l}\text { Childhood } \\
\text { in US } \\
\text { outside US }\end{array}$ & $\begin{array}{r}98.6 \% \\
1.4 \% \\
\end{array}$ & $\begin{array}{l}56.2 \% \\
43.8 \% \\
\end{array}$ & $\begin{array}{l}78.1 \% \\
21.9 \% \\
\end{array}$ \\
\hline $\begin{array}{l}\text { Adulthood } \\
\text { in US } \\
\text { outside US }\end{array}$ & $\begin{array}{c}100.0 \% \\
-\end{array}$ & $\begin{array}{l}76.9 \% \\
23.1 \% \\
\end{array}$ & $\begin{array}{l}88.8 \% \\
11.2 \% \\
\end{array}$ \\
\hline
\end{tabular}


Table 8

Professional Background

\begin{tabular}{|c|c|c|c|}
\hline $\begin{array}{l}\text { Variable } \\
\text { Name }\end{array}$ & $\begin{array}{l}\text { Anglos } \\
n=139\end{array}$ & $\begin{array}{l}\text { Eispanics } \\
n=130\end{array}$ & $\begin{array}{l}\text { Total } \\
n=269\end{array}$ \\
\hline Revenue Sector & $\mathrm{n}=121$ & $n=110$ & $n=231$ \\
\hline $\begin{array}{l}\text { manufacturing } \\
\text { services } \\
\text { government } \\
\text { agriculture } \\
\text { other } \\
\end{array}$ & $\begin{array}{r}14.9 \% \\
57.9 \% \\
9.9 \% \\
0.8 \% \\
16.5 \% \\
\end{array}$ & $\begin{array}{r}15.5 \% \\
63.6 \% \\
4.5 \% \\
3.6 \% \\
12.8 \% \\
\end{array}$ & $\begin{array}{r}15.2 \% \\
60.6 \% \\
7.4 \% \\
2.2 \% \\
14.6 \% \\
\end{array}$ \\
\hline Span & $n=120$ & $n=108$ & $n=228$ \\
\hline $\begin{array}{l}\text { yes } \\
\text { no }\end{array}$ & $\begin{array}{l}47.5 \% \\
52.5 \% \\
\end{array}$ & $\begin{array}{l}48.1 \% \\
51.9 \% \\
\end{array}$ & $\begin{array}{l}47.8 \% \\
52.2 \% \\
\end{array}$ \\
\hline Salary & $n=119$ & $n=110$ & $n=229$ \\
\hline $\begin{array}{l}<10,000 \\
<25,000 \\
<50,000 \\
<75,000 \\
<100,000 \\
>100,000 \\
\end{array}$ & $\begin{array}{r}2.5 \% \\
12.6 \% \\
59.7 \% \\
13.4 \% \\
8.4 \% \\
3.4 \% \\
\end{array}$ & $\begin{array}{r}4.5 \% \\
14.5 \% \\
55.5 \% \\
15.5 \% \\
6.4 \% \\
3.6 \% \\
\end{array}$ & $\begin{array}{r}3.5 \% \\
13.5 \% \\
57.6 \% \\
14.4 \% \\
7.4 \% \\
3.5 \% \\
\end{array}$ \\
\hline Ethcode1 & $n=121$ & $n=110$ & $n=231$ \\
\hline $\begin{array}{l}\text { yes } \\
\text { no }\end{array}$ & $\begin{array}{l}76.9 \% \\
23.1 \%\end{array}$ & $\begin{array}{l}64.5 \% \\
35.5 \%\end{array}$ & $\begin{array}{l}71.0 \% \\
29.0 \%\end{array}$ \\
\hline Ethcode2 & $n=121$ & $\mathrm{n}=110$ & $n=231$ \\
\hline $\begin{array}{l}\text { yes } \\
\text { no }\end{array}$ & $\begin{array}{l}52.1 \% \\
47.9 \%\end{array}$ & $\begin{array}{l}44.5 \% \\
55.5 \%\end{array}$ & $\begin{array}{l}48.5 \% \\
51.5 \%\end{array}$ \\
\hline
\end{tabular}


Table 9

Measurement Models

For Independent Variables:

Fit Indices and Factor Correlations (Phis)

\begin{tabular}{|l|l|}
\hline INDIVIDUALISM (COMP+EED) & COLLECTIVISM (INTER+FINT) \\
\hline chi-sq.80.08 & chi-sq.60.11 \\
(df $=54, p=0.01)$ & (df $=54, p=0.26)$ \\
cf $i=0.93$ & cfi=0.99 \\
gf $i=0.94$ & gf $i=0.95$ \\
nnf $i=0.93$ & nnf $i=0.99$ \\
phi $i=-0.20$ & phi=0.37 \\
\hline
\end{tabular}


Table 10

CFA - Individualism

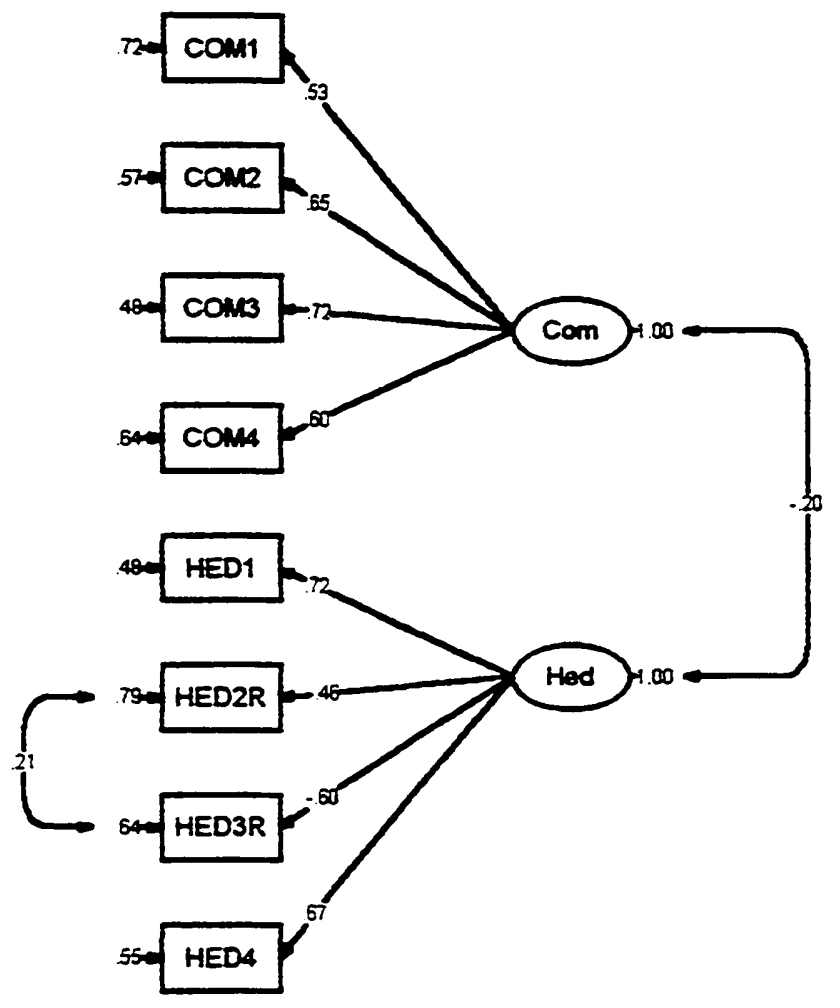


Table 10

\section{CFA - Individualism $t$-values}

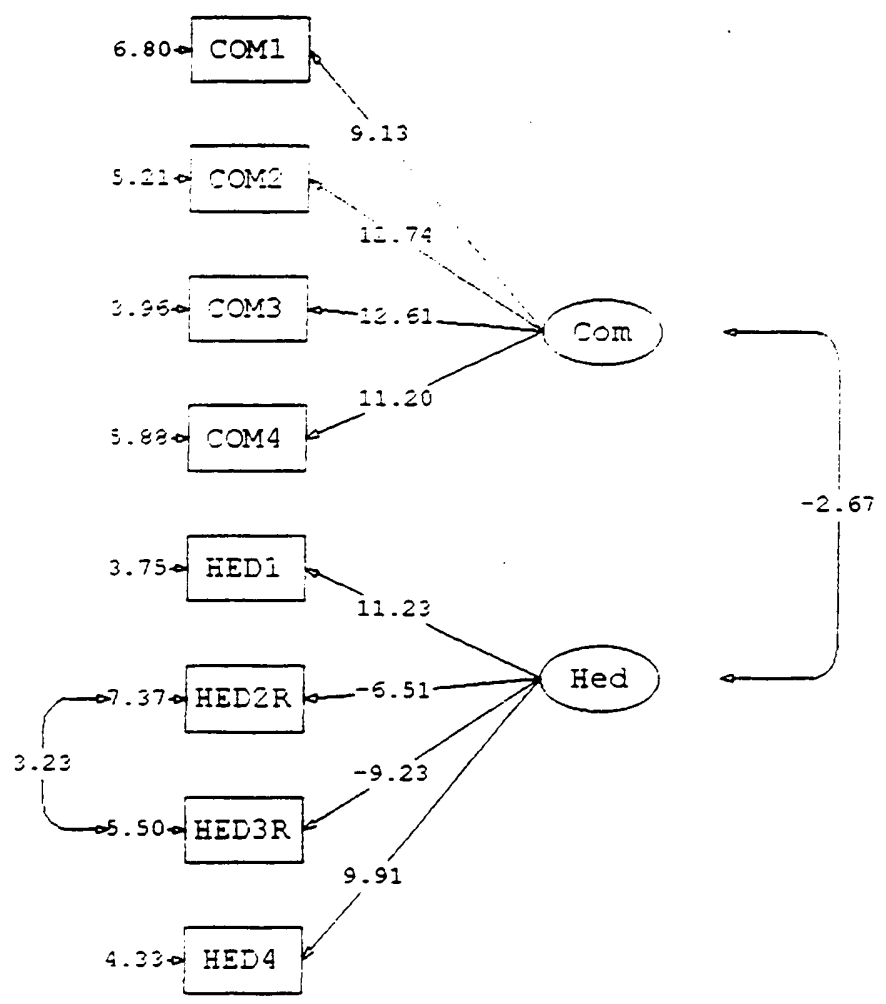




\section{CFA - Collectivism}

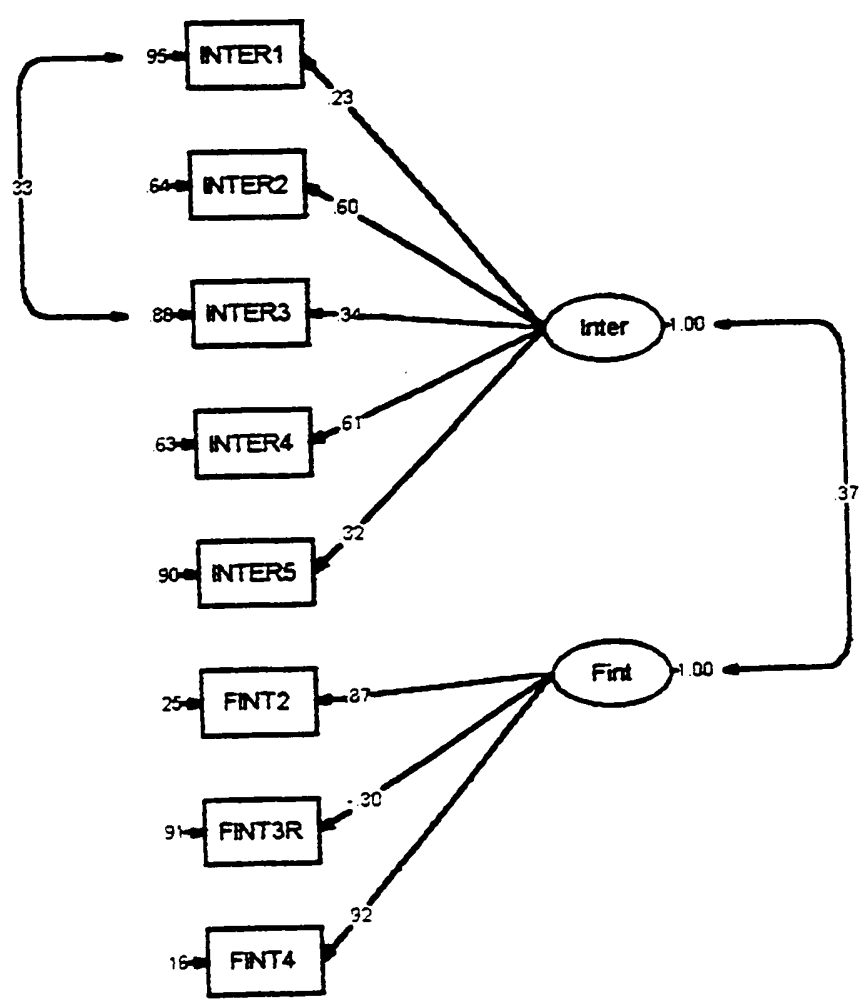




\section{Table 11}

\section{CFA - Collectivism t-vahues}

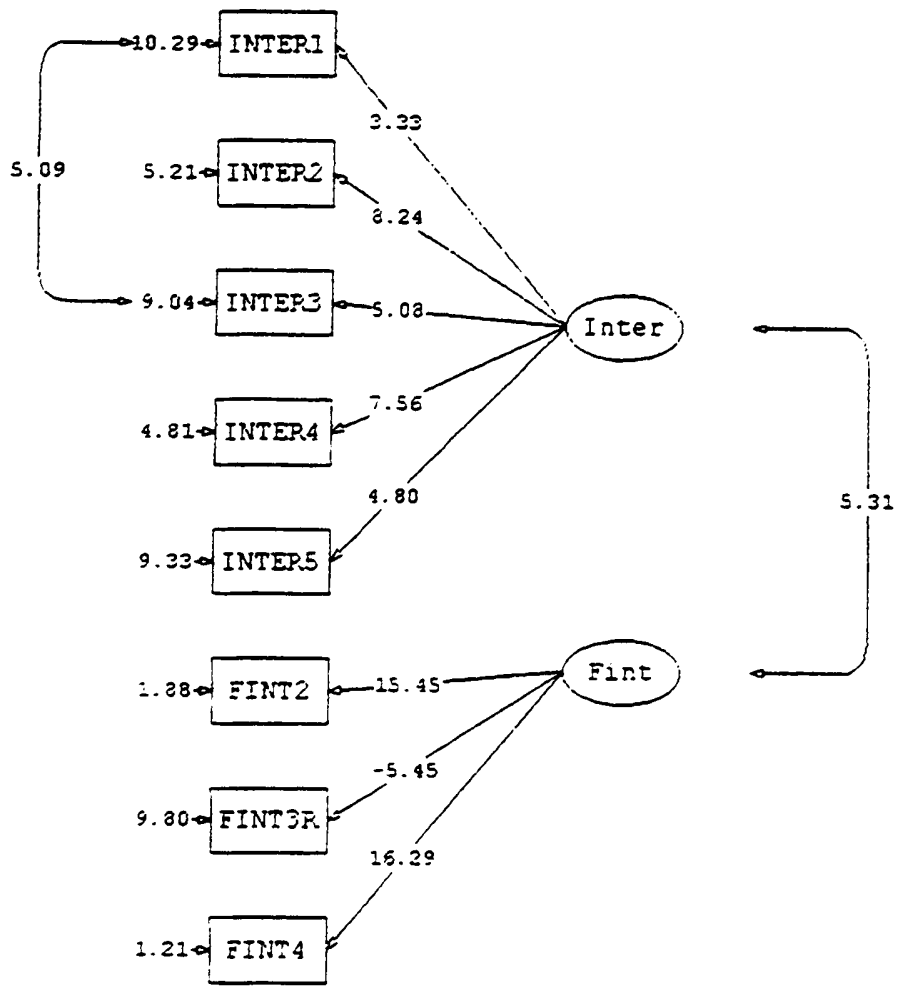


Table 12

Measurement Models

For Dependent Variables:

Fit Indices

\begin{tabular}{|c|c|c|}
\hline CONTIEST: & $\begin{array}{l}\text { No Attributions } \\
\text { (inda-d) }\end{array}$ & $\begin{array}{l}\text { RO Attributions } \\
\text { (cola-d) }\end{array}$ \\
\hline $\begin{array}{l}\text { SCENARIO } 1 \\
\text { ROLLFAST BYCICLE } \\
\text { CO. }\end{array}$ & $\begin{array}{l}\text { chi-sq. } 15.24 \\
(d f=11, p=0.17) \\
\text { cf } i=0.95 \\
\text { gf } i=0.98 \\
\text { nnf } i=0.95\end{array}$ & $\begin{array}{l}\text { chi-sq.6.39 } \\
(d f=12, p=0.89) \\
\text { cf } i=1.00 \\
\text { gf } i=0.98 \\
\text { nnf } i=1.10\end{array}$ \\
\hline $\begin{array}{l}\text { SCENARIO } 2 \\
\text { TANGY SPICES }\end{array}$ & $\begin{array}{l}\text { chi-sq.11.56 } \\
(\mathrm{df}=12, p=0.48) \\
\text { cf } i=1.00 \\
\text { gf } i=0.99 \\
\text { nnf } i=1.00\end{array}$ & $\begin{array}{l}\text { chi-sq.4.16 } \\
(d f=12, p=0.98) \\
\text { cf } i=1.00 \\
\text { gf } i=0.99 \\
\text { nnf } i=1.03\end{array}$ \\
\hline $\begin{array}{l}\text { SCENARIO } 3 \\
\text { MICROCOMPUTER FIRM }\end{array}$ & $\begin{array}{l}\text { chi-sq.2.16 (3) } \\
(\mathrm{df}=6, p=0.90) \\
\text { cf } i=1.00 \\
\text { gf } i=0.99 \\
\text { nnf } i=1.07\end{array}$ & $\begin{array}{l}\text { chi-sq. } 28.81 \\
(d f=12, p=0.0042 *) \\
\text { cfi=0.94 } \\
\text { gf } i=0.96 \\
\text { nnf } i=0.94\end{array}$ \\
\hline $\begin{array}{l}\text { SCENARIO } 4 \\
\text { J\&P PUBLISHING CO. }\end{array}$ & $\begin{array}{l}\text { chi-sq.15.24 (3) } \\
(d f=6, p=0.17) \\
\text { cf } i=0.99 \\
\text { gf } i=0.98 \\
\text { nnf } i=0.99\end{array}$ & $\begin{array}{l}\operatorname{chi} i-s q \cdot 7.50 \\
(d f=11, p=0.76) \\
\operatorname{cf} i=1.00 \\
\operatorname{gf} i=0.99 \\
\operatorname{nnf} i=1.02\end{array}$ \\
\hline
\end{tabular}

"p-values given with more that 2 cecieals 
Table 13

CFA - MO - Scenario 1

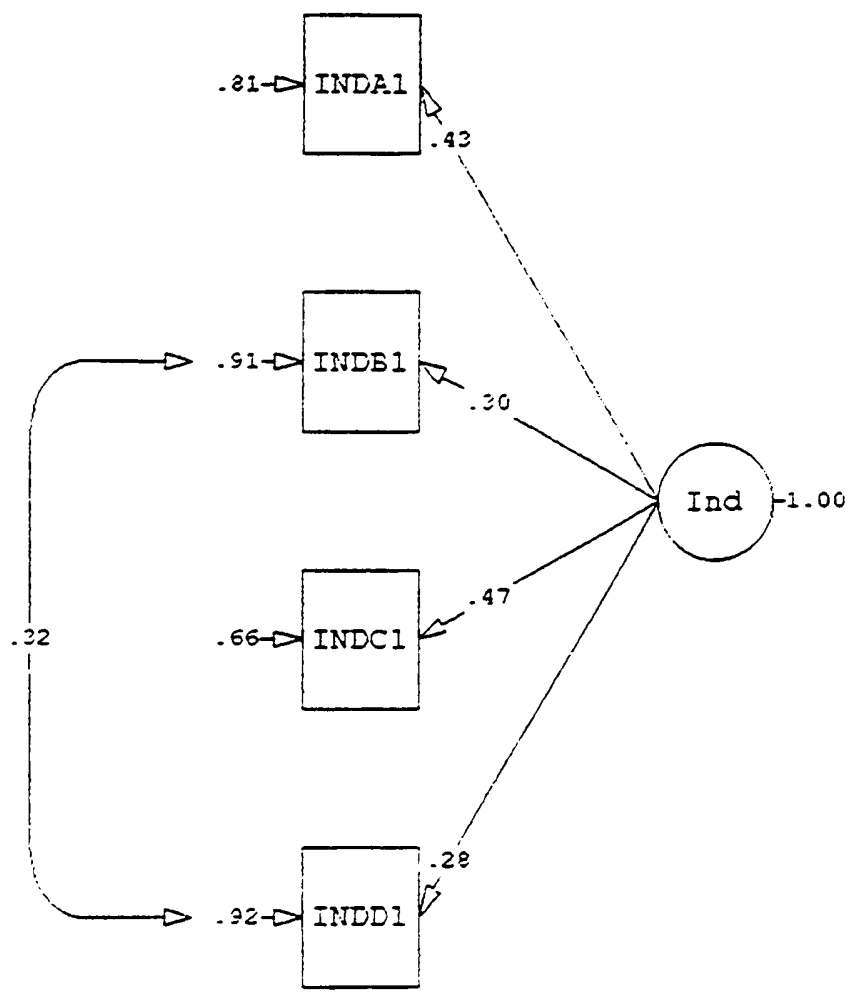

ROLLFAST BICYCLE CO. 
Table 13

CFA - MO - Scenario 1

t-values

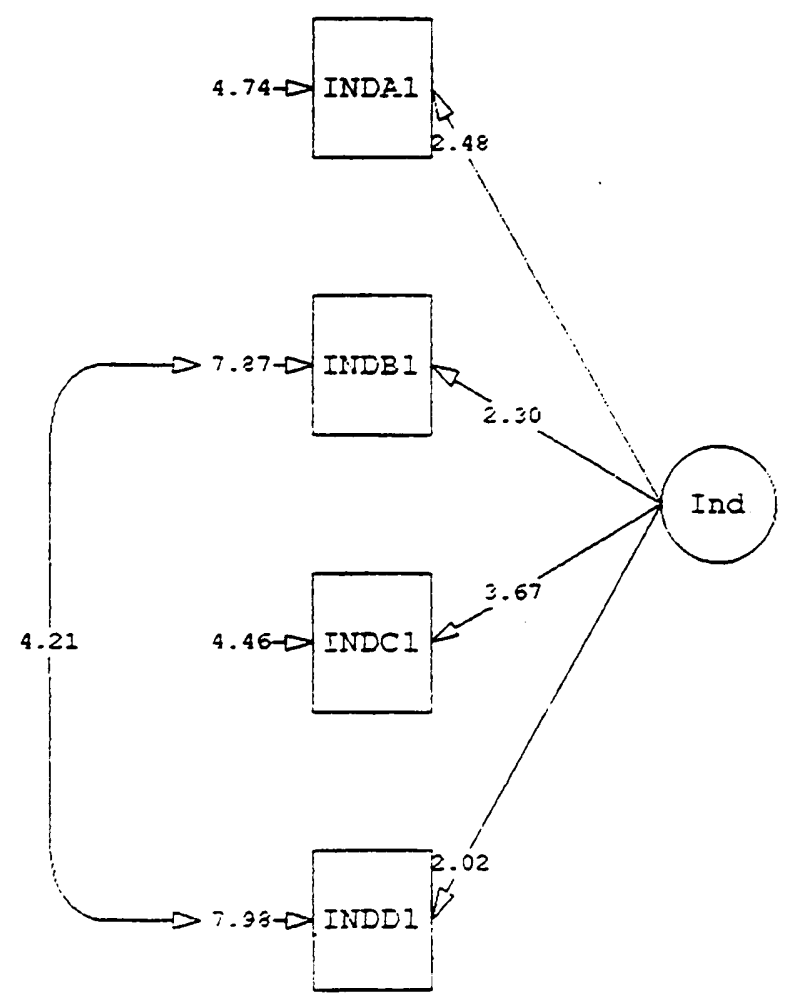

ROLLFAST BICYCLE CO. 


\section{Table 14}

CFA - RO - Scenario 1

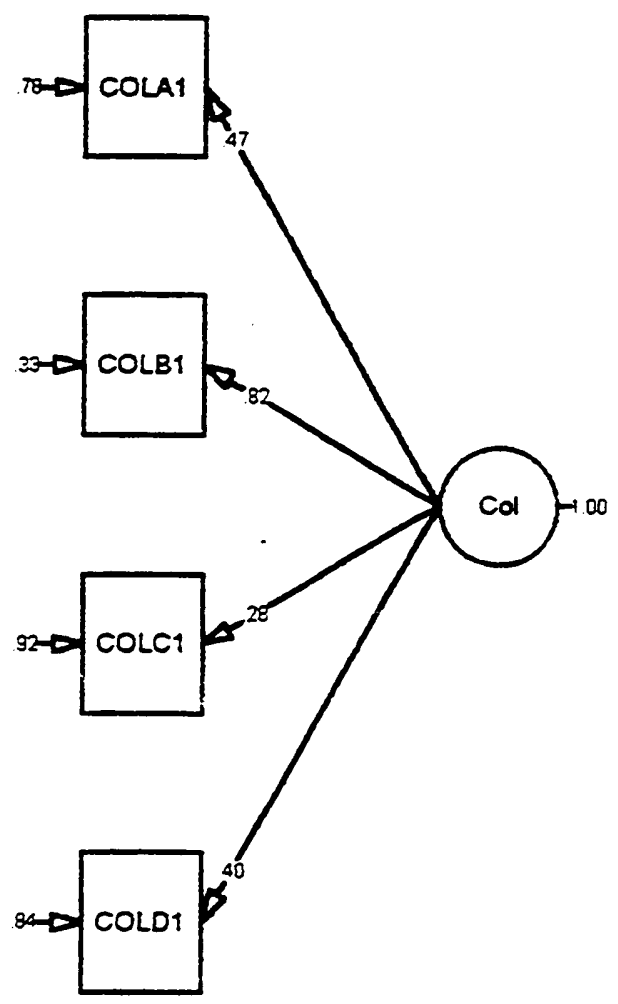

ROLLFAST BICYCLE CO. 


\section{Table 14}

\section{CFA - RO - Scenario 1}

\section{t-values}

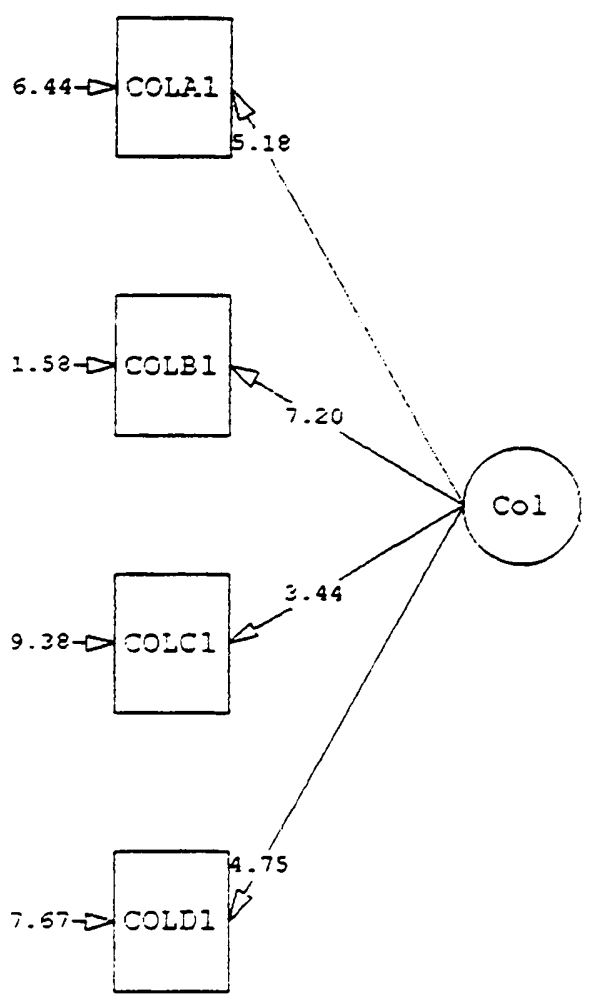

ROLLFAST BICYCLE CO. 
Table 15

CFA - MO - Scenario 2

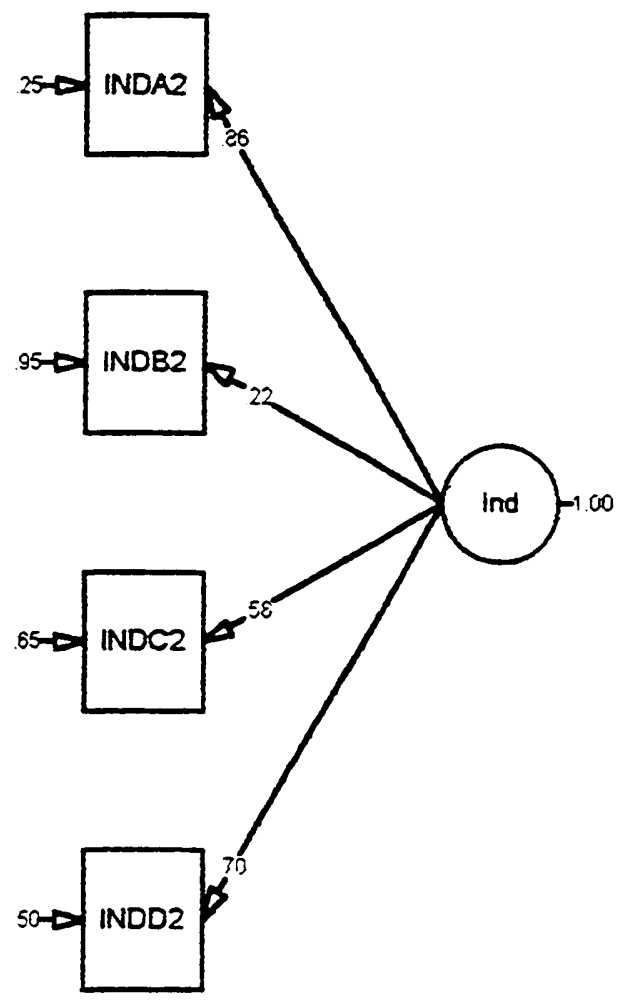

TANGY SPICES 


\section{Table 15}

CFA - MO - Scenario 2

$\mathrm{t}$-values

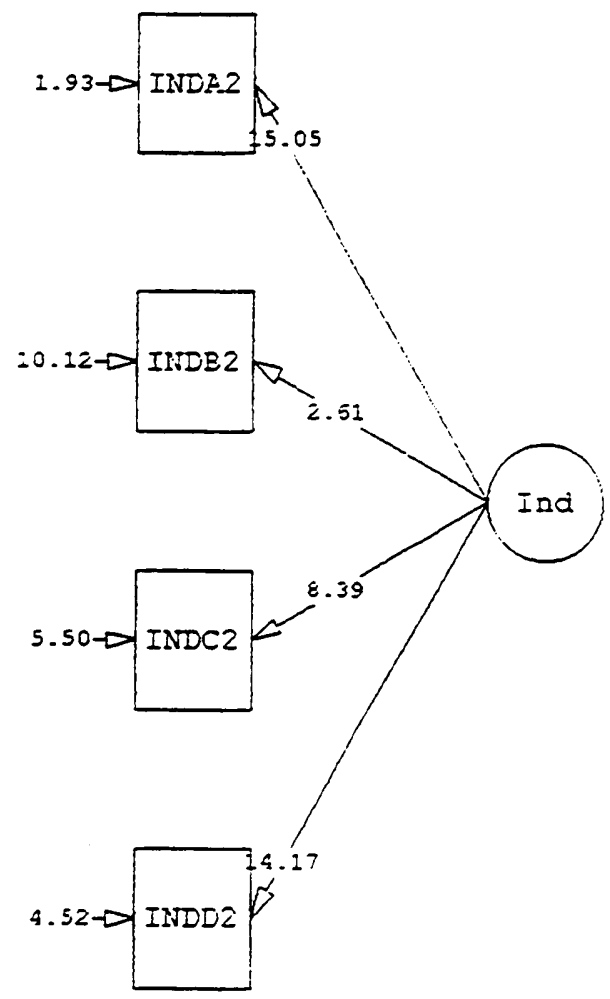

TANGY SPICES 
CFA - RO - Scenario 2

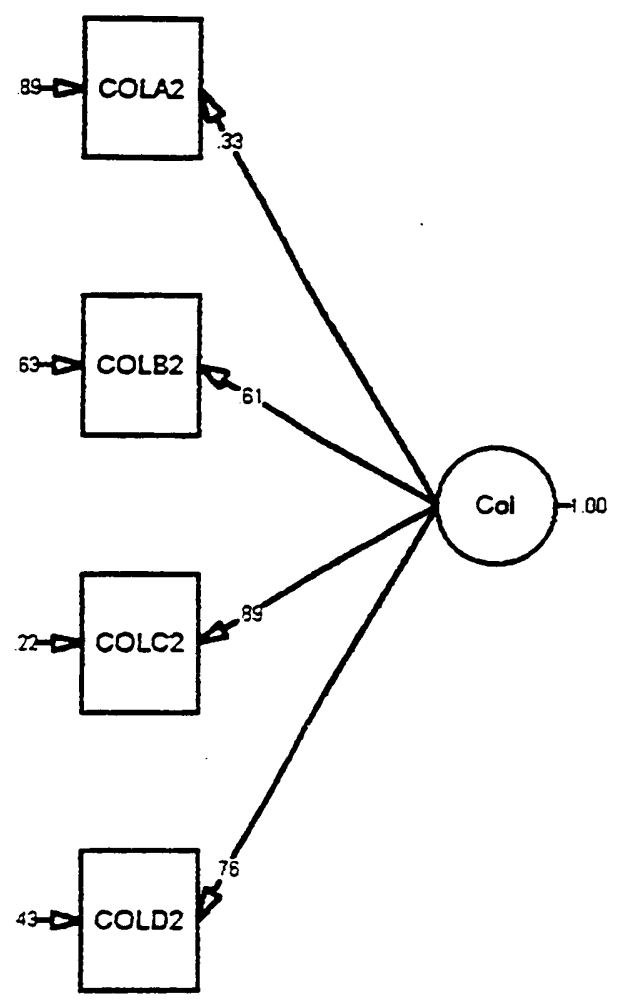

TANGY SPICES 


\section{Table 16}

CFA - RO - Scenario 2

$\mathrm{t}$-vahues

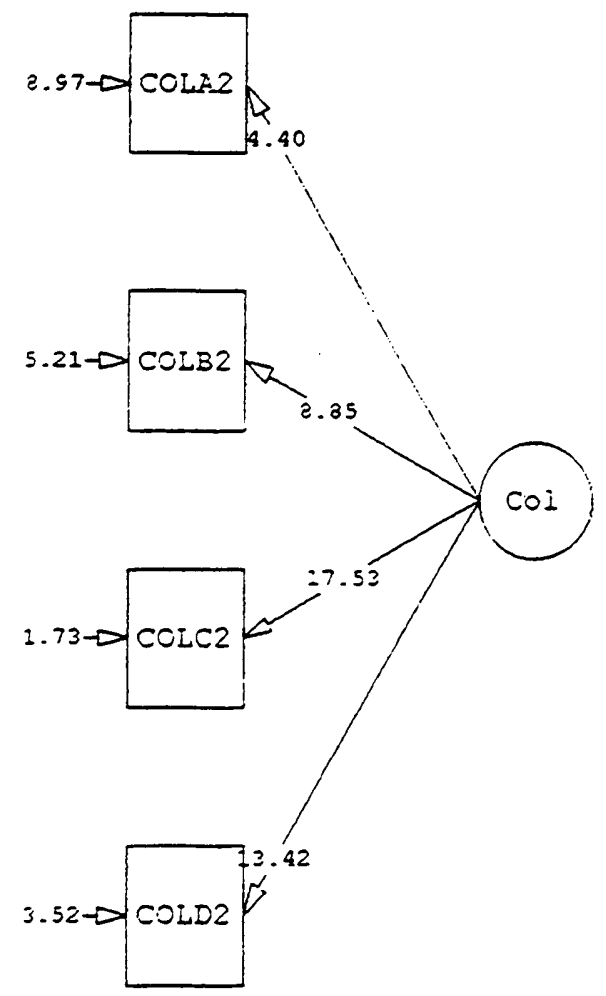

TANGY SPICES 
Table 17

CFA - MO - Scenario 3

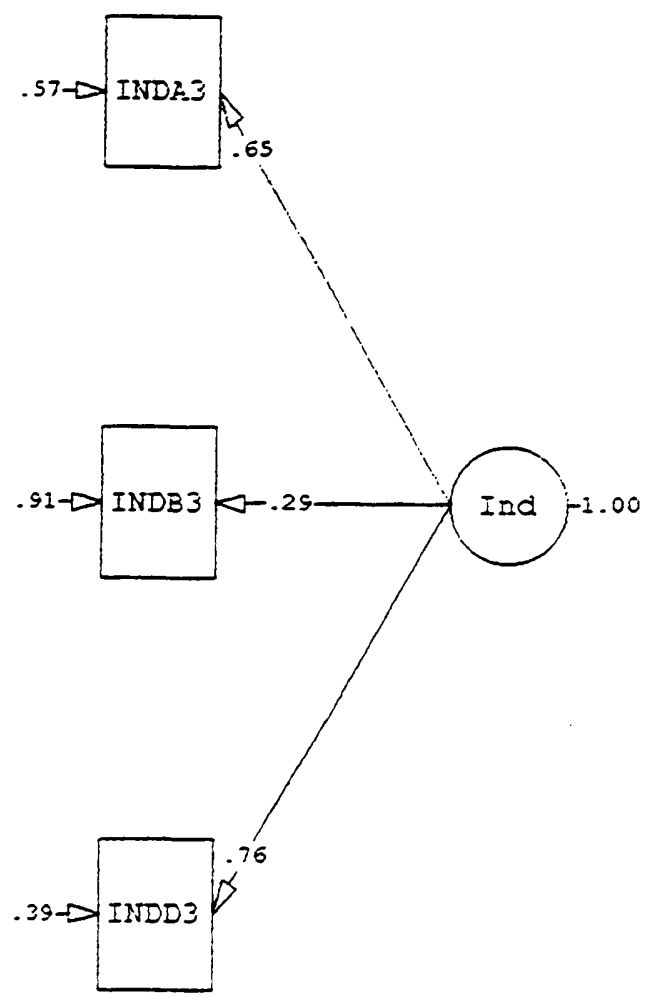

MICROCOMPUTER FIRM 


\section{Table 17}

\section{CFA - MO - Scenario 3}

\section{t-values}

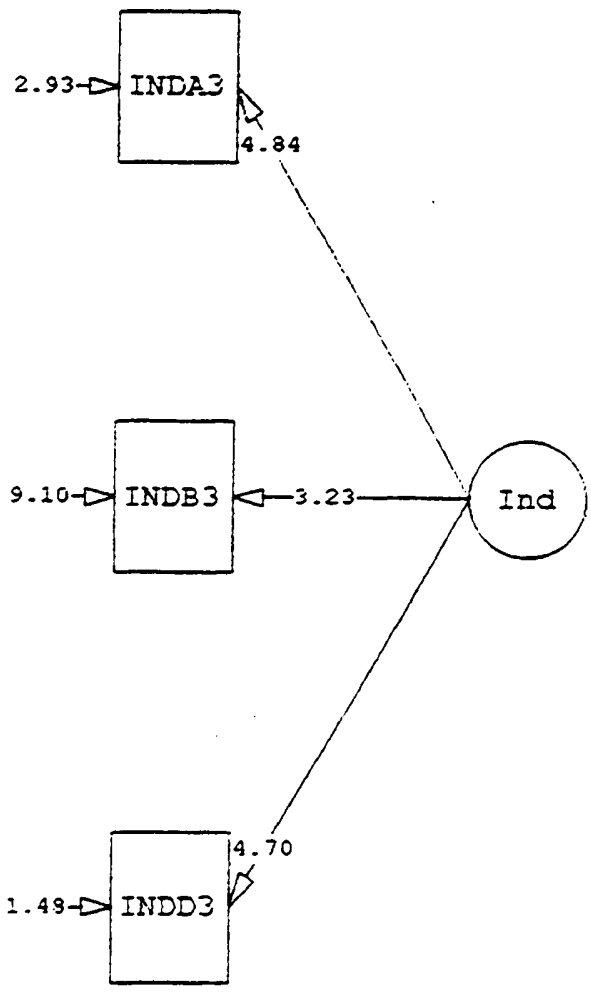

MICROCOMPUTER FIRM 


\section{Table 18}

CFA - RO - Scenario 3

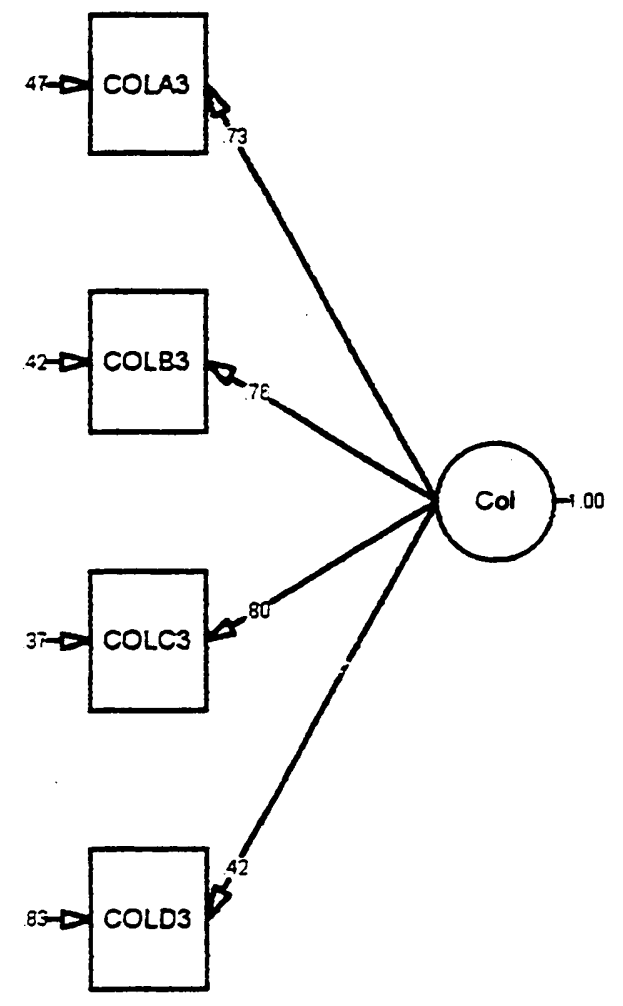

MICROCOMPUTER FIRM 
Table 18

CFA - RO - Scenario 3

t-values

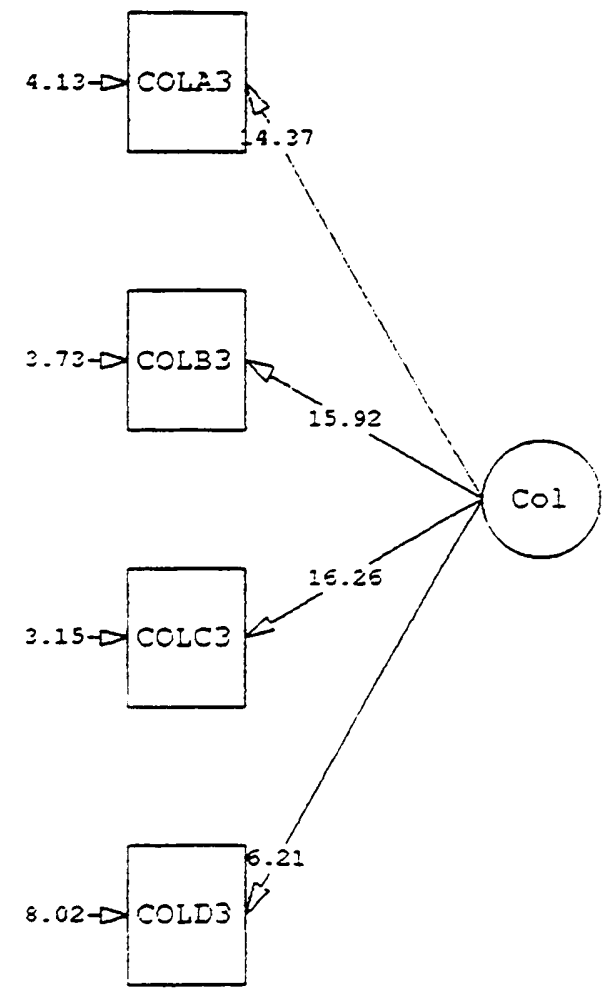

MICROCOMPUTER FIRM 


\section{CFA - MO - Scenario 4}

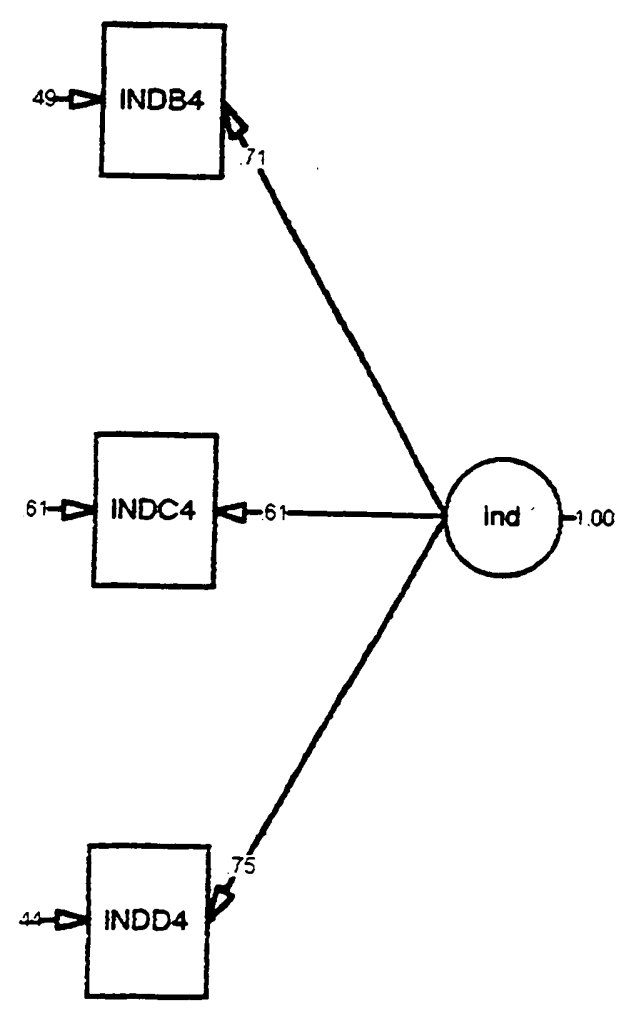

J\&P PUBLISHING CO. 
CFA - MO - Scenario 4 t-values

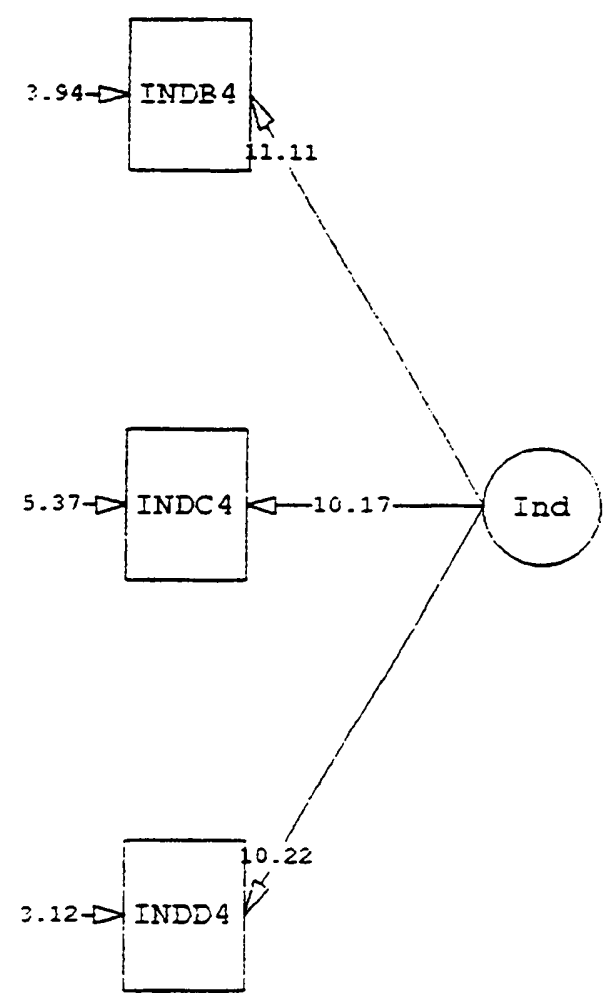

J\&P PUBLISHING CO. 


\section{Table 20}

CFA - RO - Scenario 4

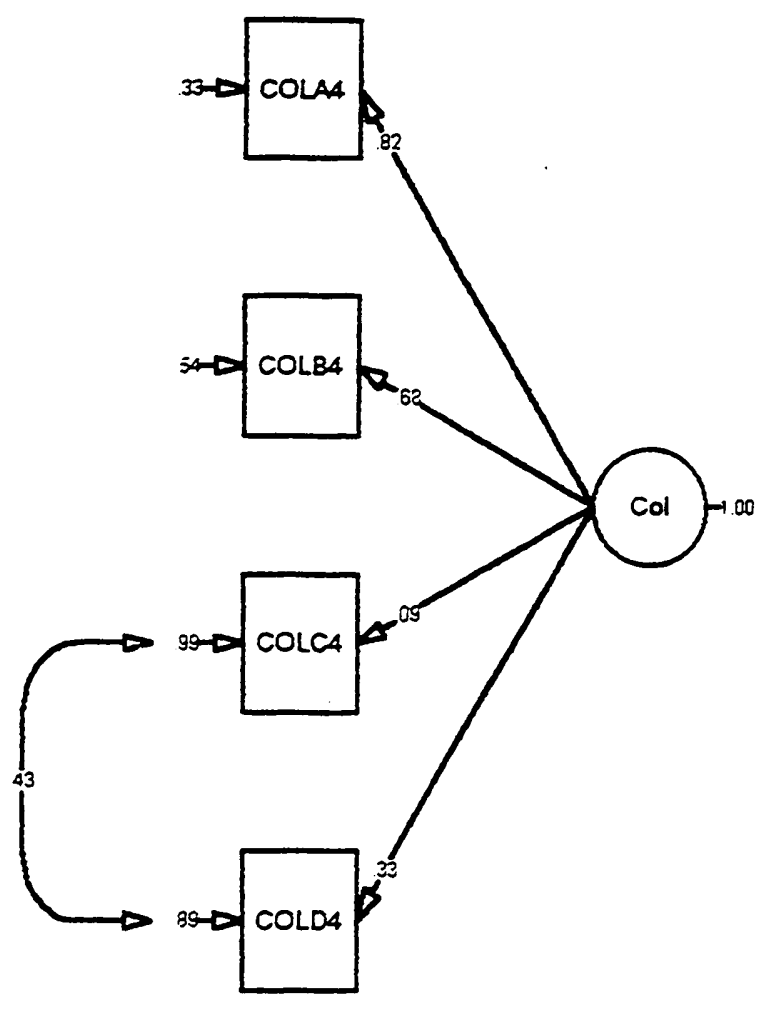

J\&P PUBLISHING CO. 
Table 20

CFA - RO - Scenario 4

t-values

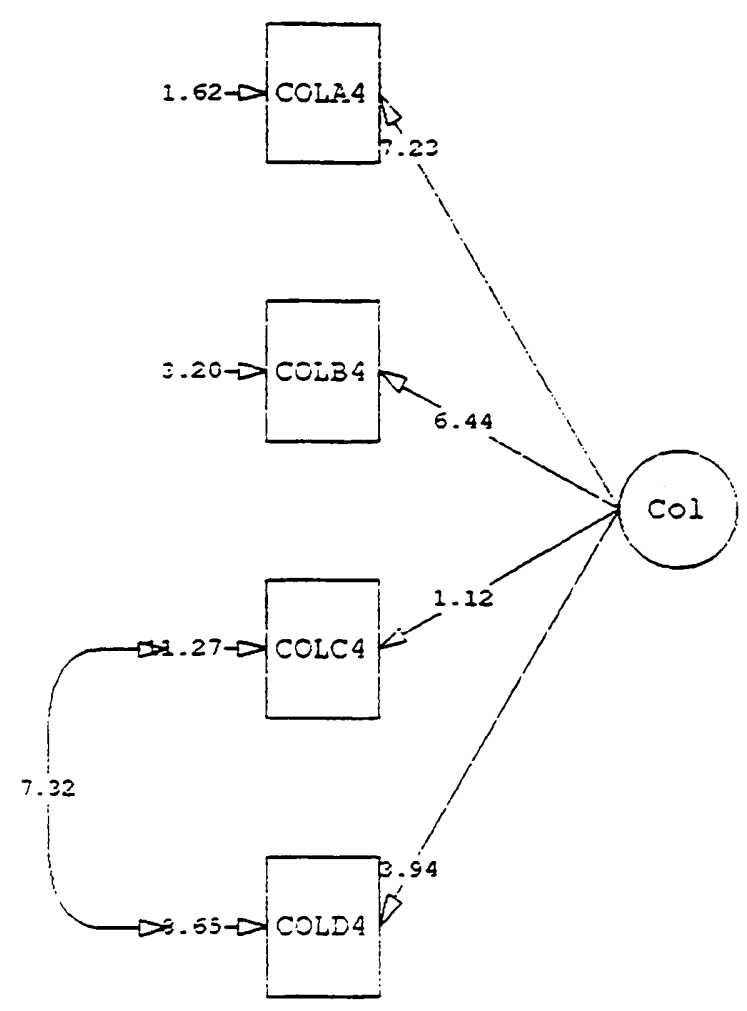

J\&P PUBLISHING CO. 
Table 21

Full Models

Fit Indices and R-squares

\begin{tabular}{|c|c|c|}
\hline COATEST: & IXDIVIDUALISY-MO & COLLECTIVISM-RO \\
\hline $\begin{array}{c}\text { SCENARIO I } \\
\text { ROLLFAST BYCICLE } \\
\text { CO. }\end{array}$ & $\begin{array}{l}\text { chi-sq. } 279.72 \\
(d f=125, p=0.00) \\
\text { cf } i=0.86 \\
\text { gf } i=0.88 \\
\text { nnf } i=0.85 \\
\text { R-sq. (A) }=0.10 \\
\text { R-sq. (H) }=0.11\end{array}$ & $\begin{array}{l}\text { chi-sq. } 236.85 \\
(d f=126, p=0.00) \\
\text { cf } i=0.91 \\
\text { gf } i=0.91 \\
\operatorname{nnf} i=0.91 \\
R-s q \cdot(A)=0.27 \\
R-s q \cdot(H)=0.26\end{array}$ \\
\hline $\begin{array}{l}\text { SCENARIO } 2 \\
\text { TANGY SPICES }\end{array}$ & $\begin{array}{l}\text { chi-sq. } 261.32 \\
(d f=126, p=0.00) \\
\text { cf } i=0.91 \\
\text { gf } i=0.89 \\
\text { nnfi=0.91 } \\
\text { R-sq. (A)=0.005* } \\
\text { R-sq. (H)=0.078* }\end{array}$ & $\begin{array}{l}\text { chi-sq. } 250.64 \\
(d f=126, p=0.00) \\
\text { cfi=0.92 } \\
\text { gfi=0.89 } \\
\text { nnf } i=0.91 \\
\text { R-sq. (A) }=0.22 \\
\text { R-sq. (I) }=0.23\end{array}$ \\
\hline MICROCOMPUTER FIRM & $\begin{array}{l}\text { chi-sq. } 198.81 \\
(d f=105, p=0.00) \\
\text { cf } i=0.90 \\
g f i=0.92 \\
\text { nnfi }=0.89 \\
R-s q \cdot(A)=0.076 * \\
R-s q .(B)=0.15\end{array}$ & $\begin{array}{l}\text { chi-sq. } 288.80 \\
(d f=126, p=0.00) \\
\text { cf } i=0.91 \\
\text { gf } i=0.89 \\
\operatorname{nnf} i=0.90 \\
R-s q \cdot(A)=0.049 * \\
R-s q .(H)=0.26\end{array}$ \\
\hline J\&P PUBLISHING CO. & $\begin{array}{l}\text { chi-sq. } 187.82 \\
(\mathrm{~d} f=104, p=0.00) \\
\text { cf } i=0.89 \\
\text { gfi=0.90 } \\
\text { nnfi } i=0.89 \\
\text { R-sq. }(\mathrm{A})=0.083^{*} \\
\text { R-sq. }(H)=0.17\end{array}$ & $\begin{array}{l}\text { chi-sq. } 237.19 \\
(\mathrm{df}=125, p=0.00) \\
\text { cf } i=0.92 \\
\text { gf } i=0.89 \\
\text { nnf } i=0.92 \\
\text { R-sq. }(A)=0.021 * \\
\text { R-sq. (H) }=0.14\end{array}$ \\
\hline
\end{tabular}


Table 22

Full Model Individualism - MO

Scenario 1 - Anglos

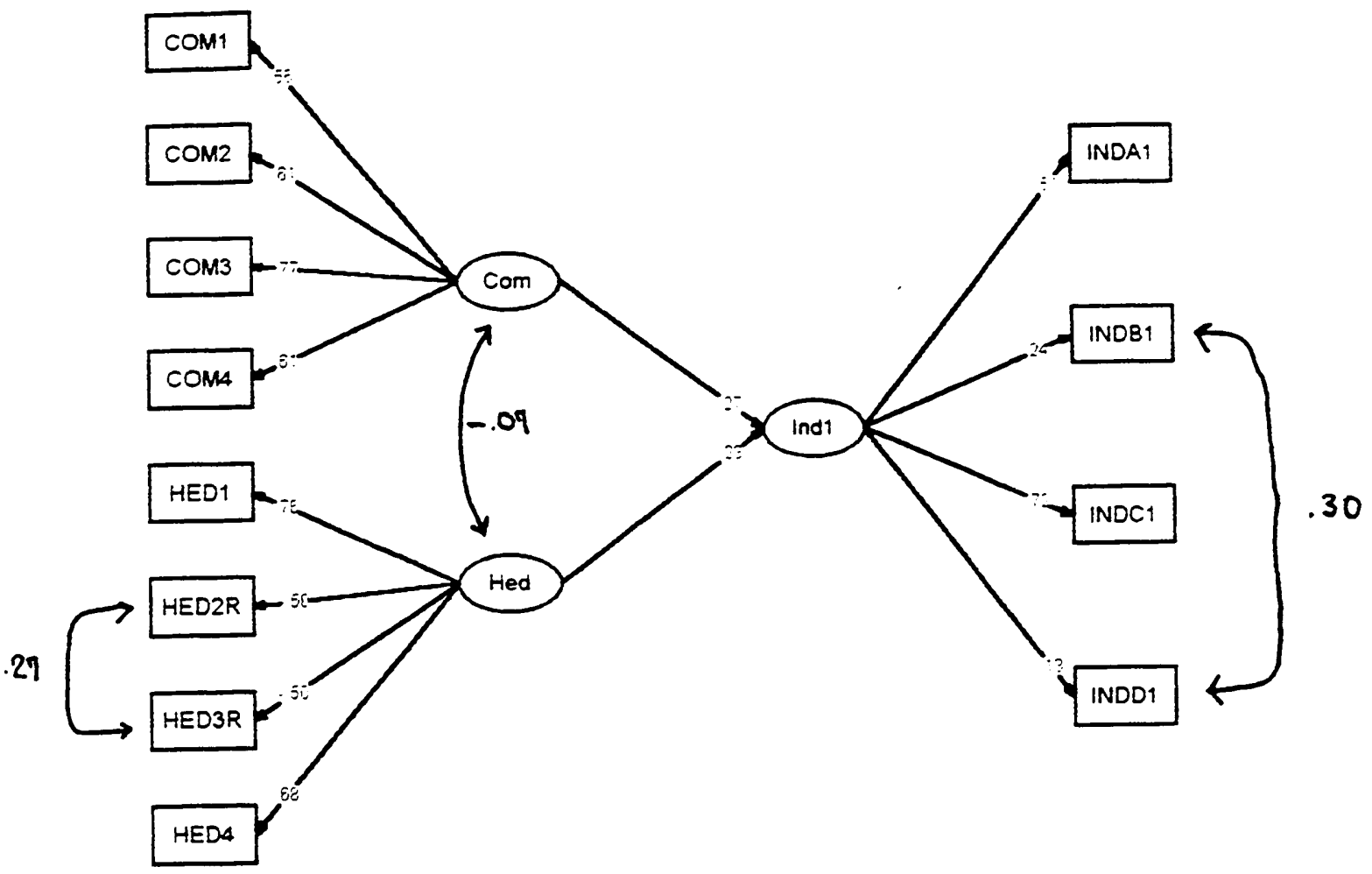

ROLLFAST BICYCLE CO. 
Table 22

Full Model Individualism - MO

Scenario 1 - Anglos

$\mathrm{t}$-values

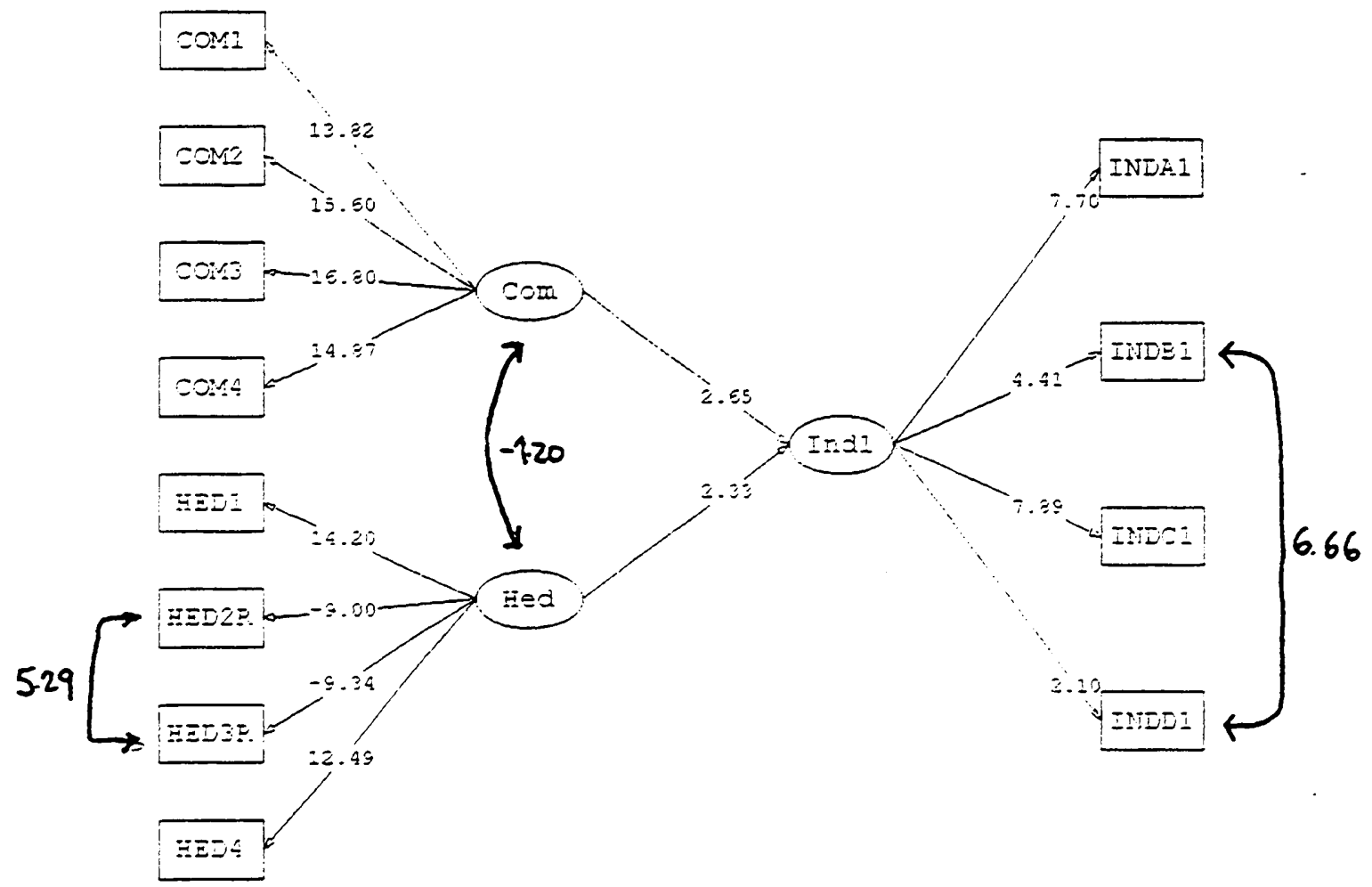

ROLLFAST BICYCLE CO. 
Table 23

Full Model Individualism - MO

Scenario 1 - Hispanics

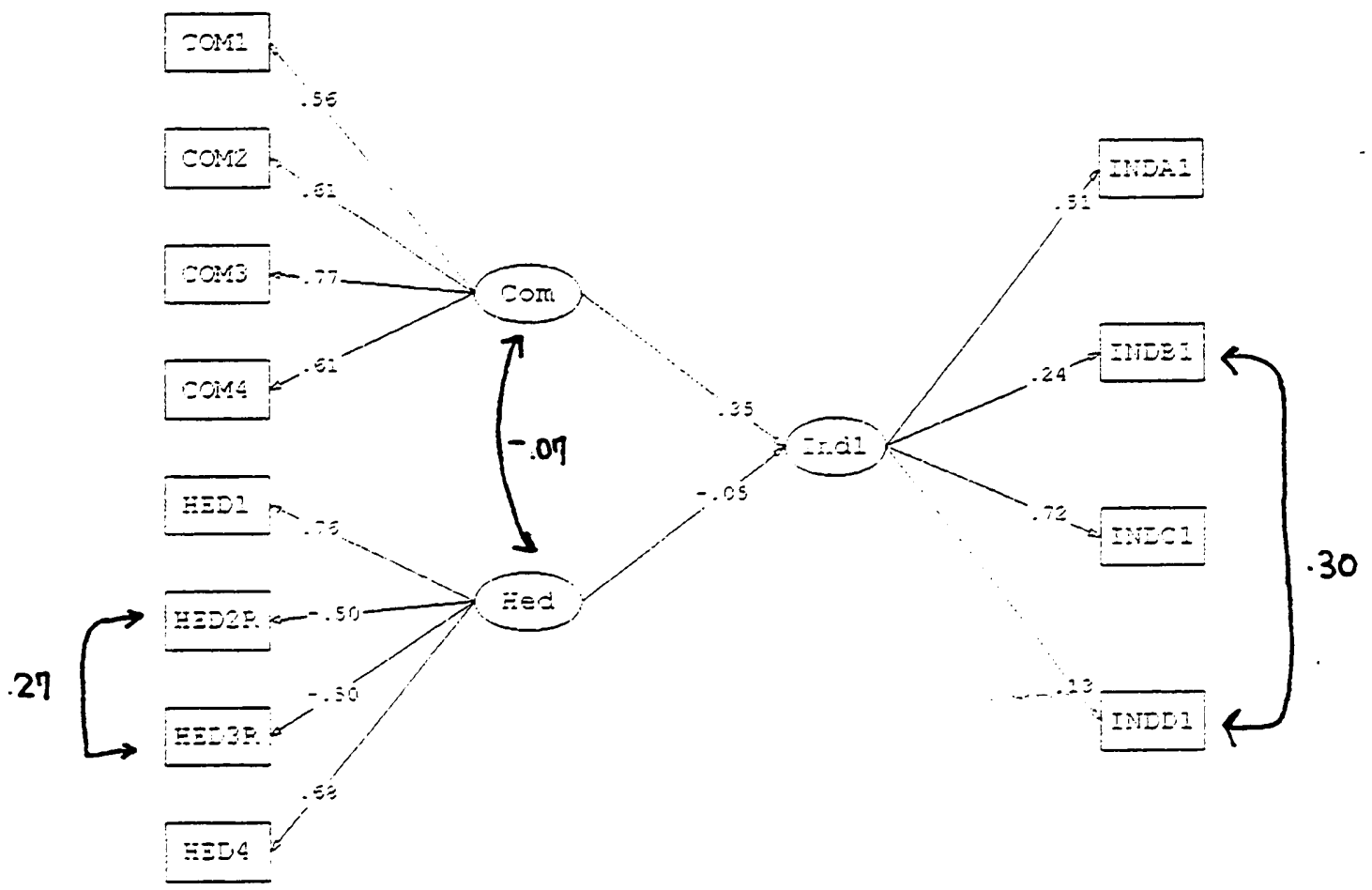

ROLLFAST BICYCLE CO. 
Table 23

Full Model Individualism - MO

Scenario 1 - Hispanics

$\mathrm{t}$-values

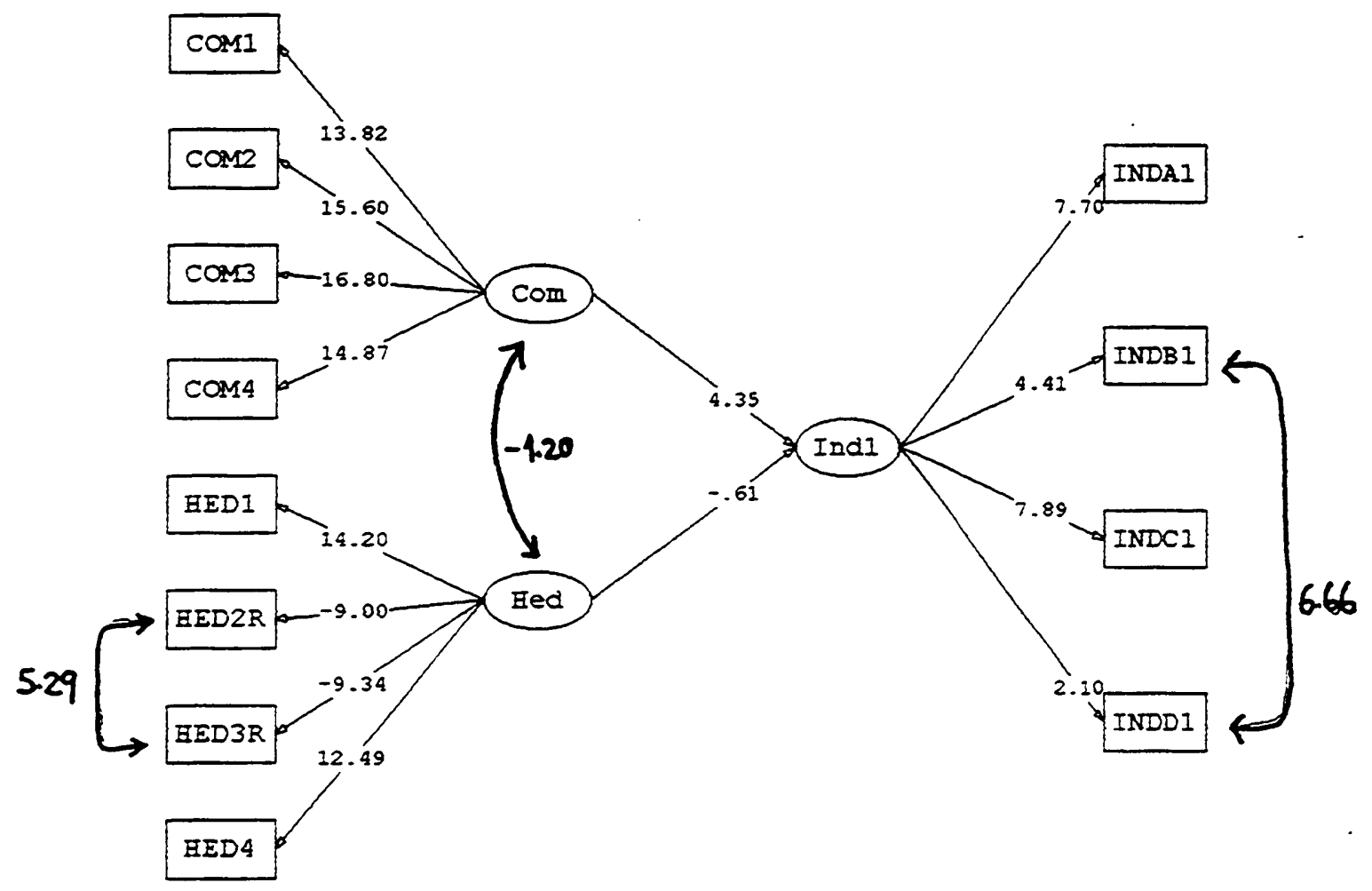

ROLLFAST BICYCLE CO. 
Table 24

Full Model Collectivism - RO

Scenario 1 - Anglos

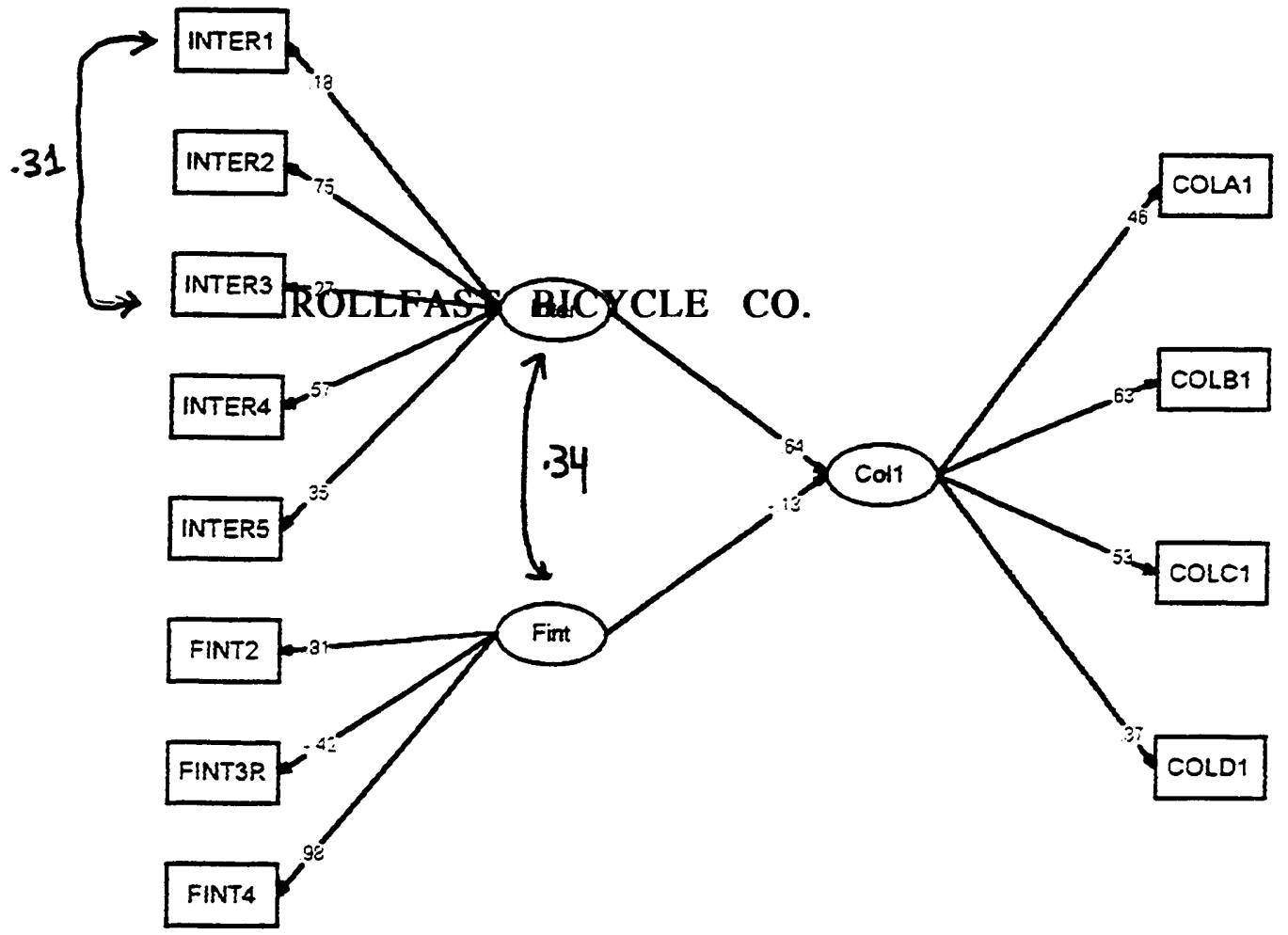


Full Model Collectivism - RO

Scenario 1 - Anglos

$\mathrm{t}$-values

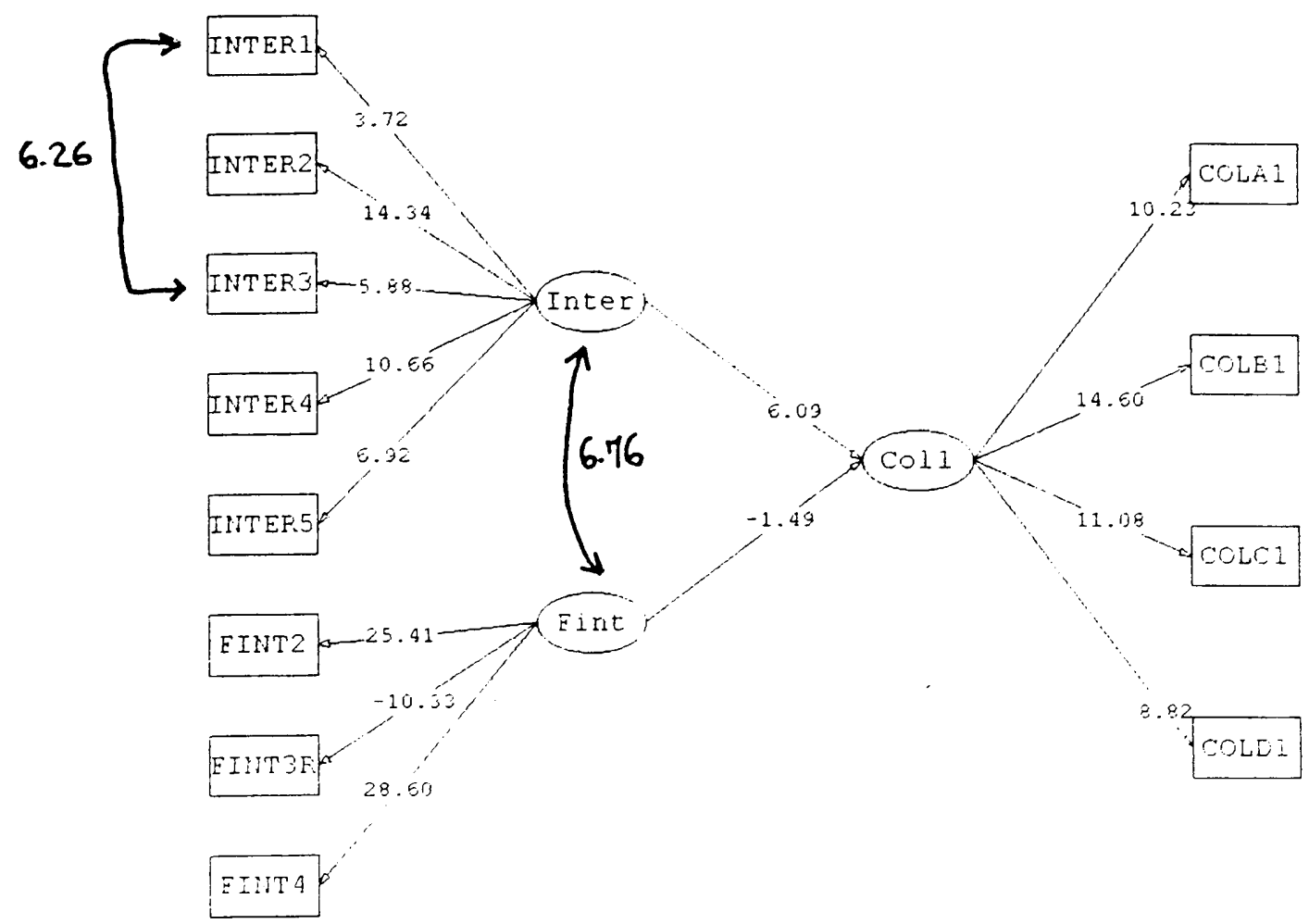




\section{Full Model Collectivism - RO}

Scenario 1 - Hispanics

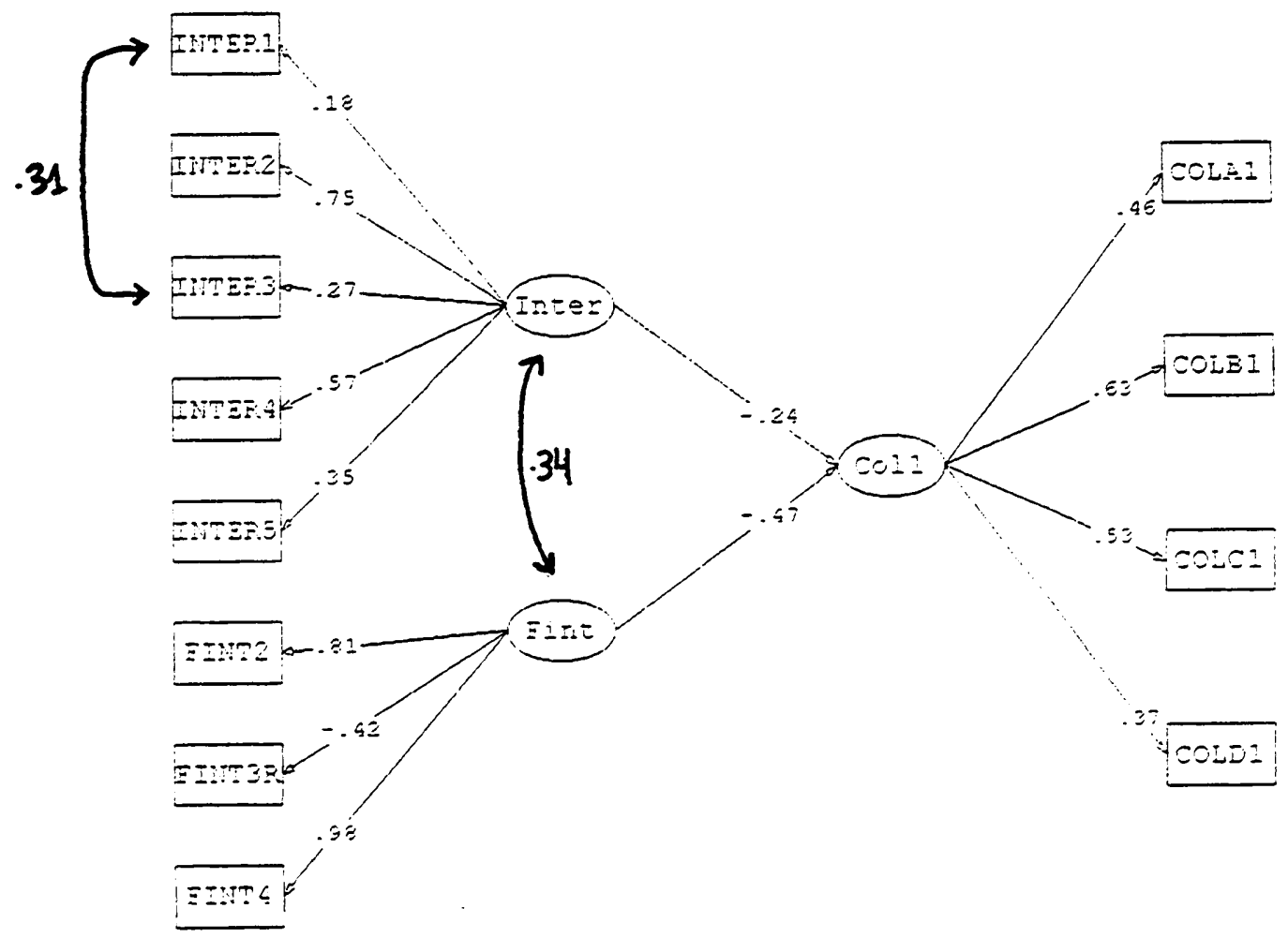

ROLLFAST BICYCLE CO. 
Table 25

Full Model Collectivism - RO

Scenario 1 - Hispanics

$t$-values

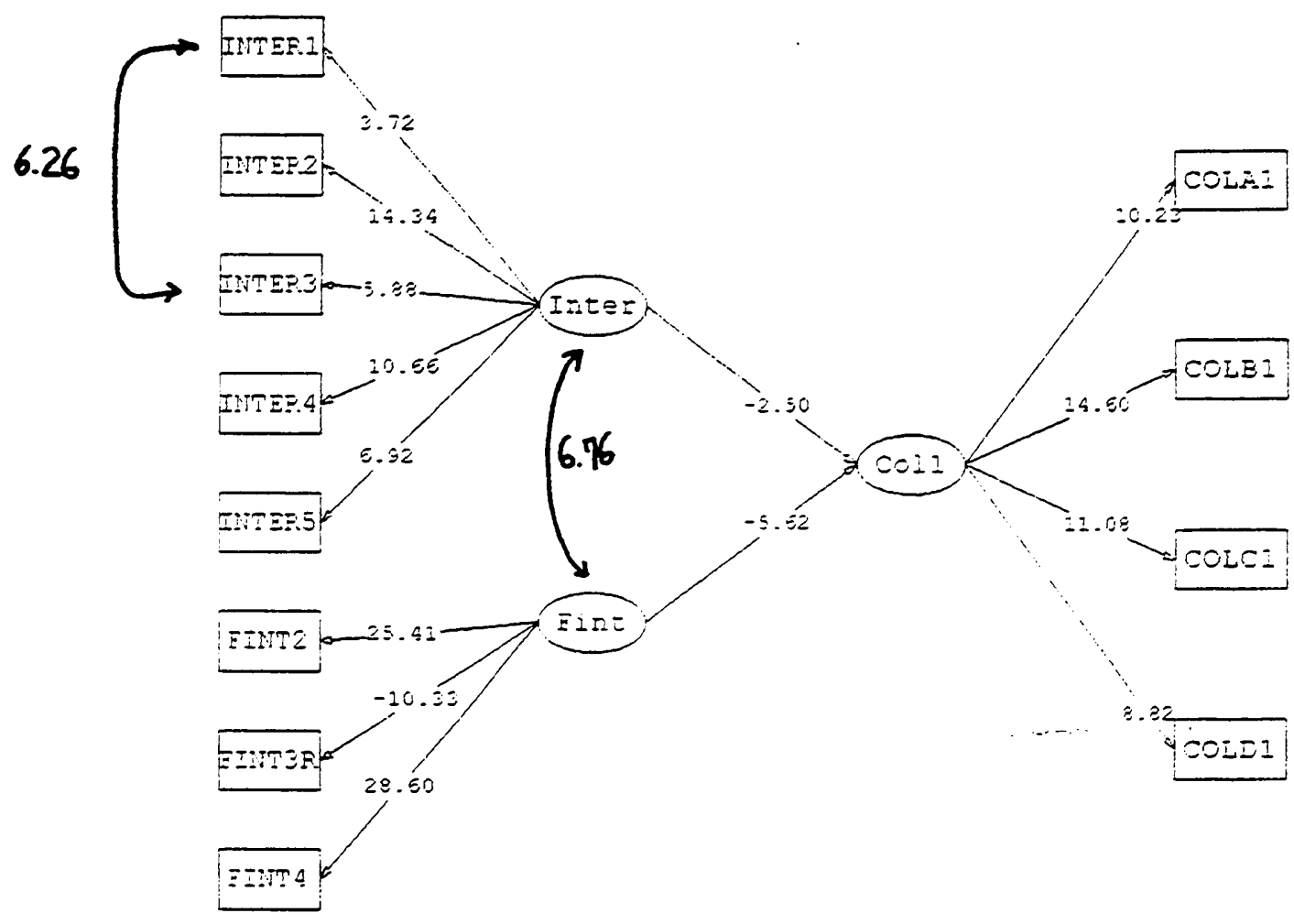

ROLLFAST BICYCLE CO. 
Table 26

Full Model Individualism - RO

Scenario 2 - Anglos

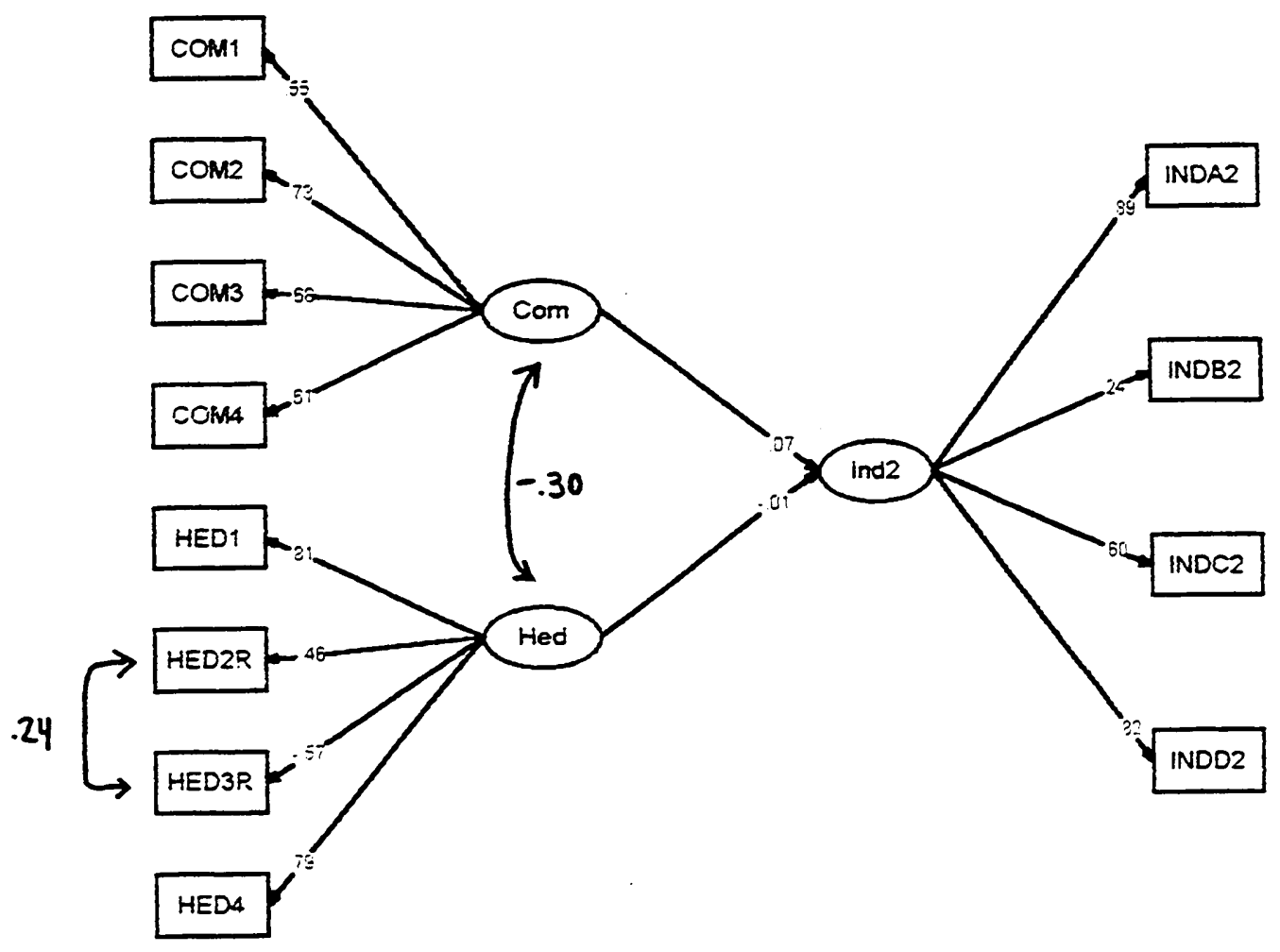

TANGY SPICES 
Table 26

Full Model Individualism - RO

Scenario 2 - Anglos

t-values

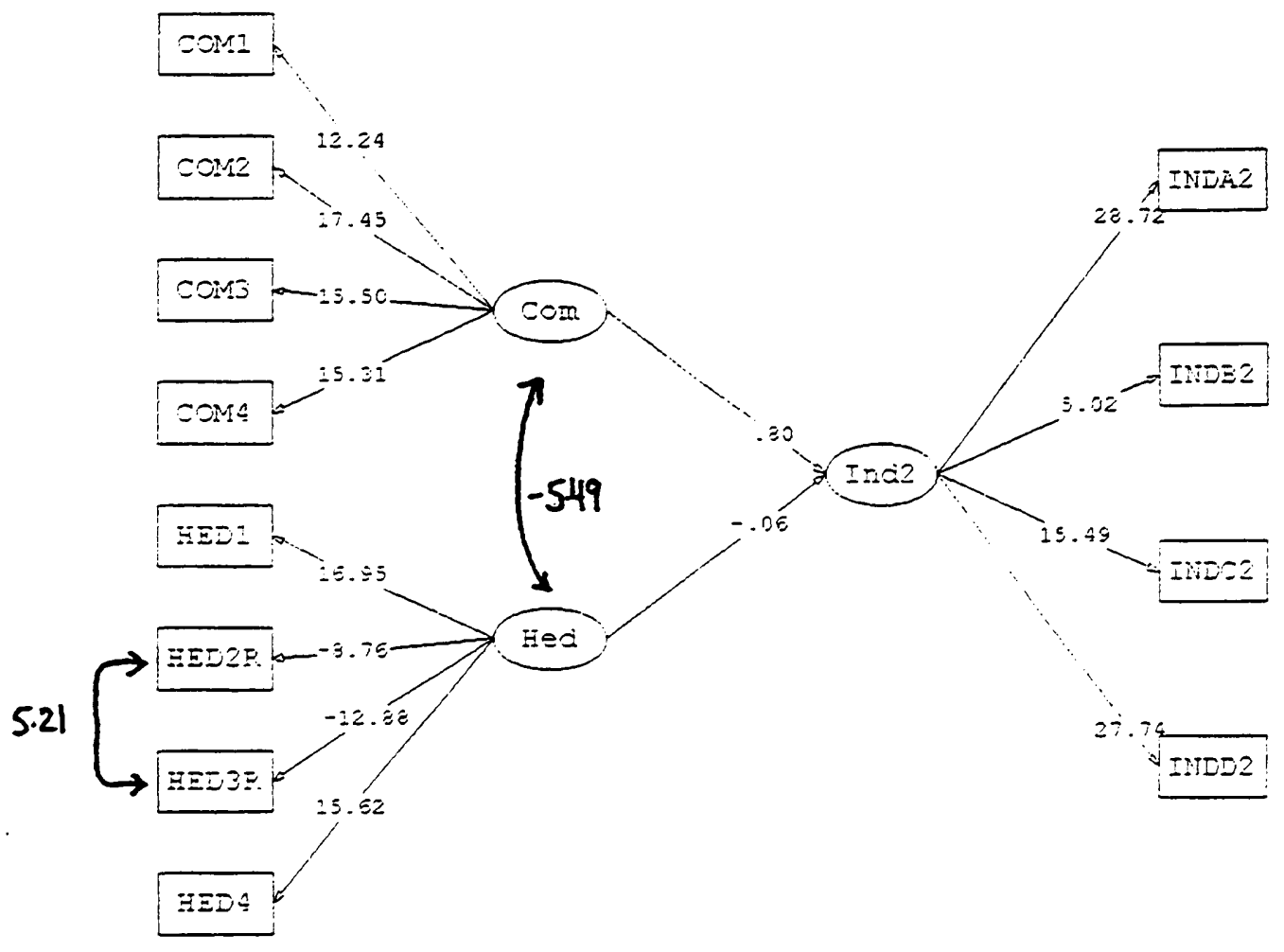

TANGY SPICES 
Full Model Individualism - RO

Scenario 2 - Hispanics

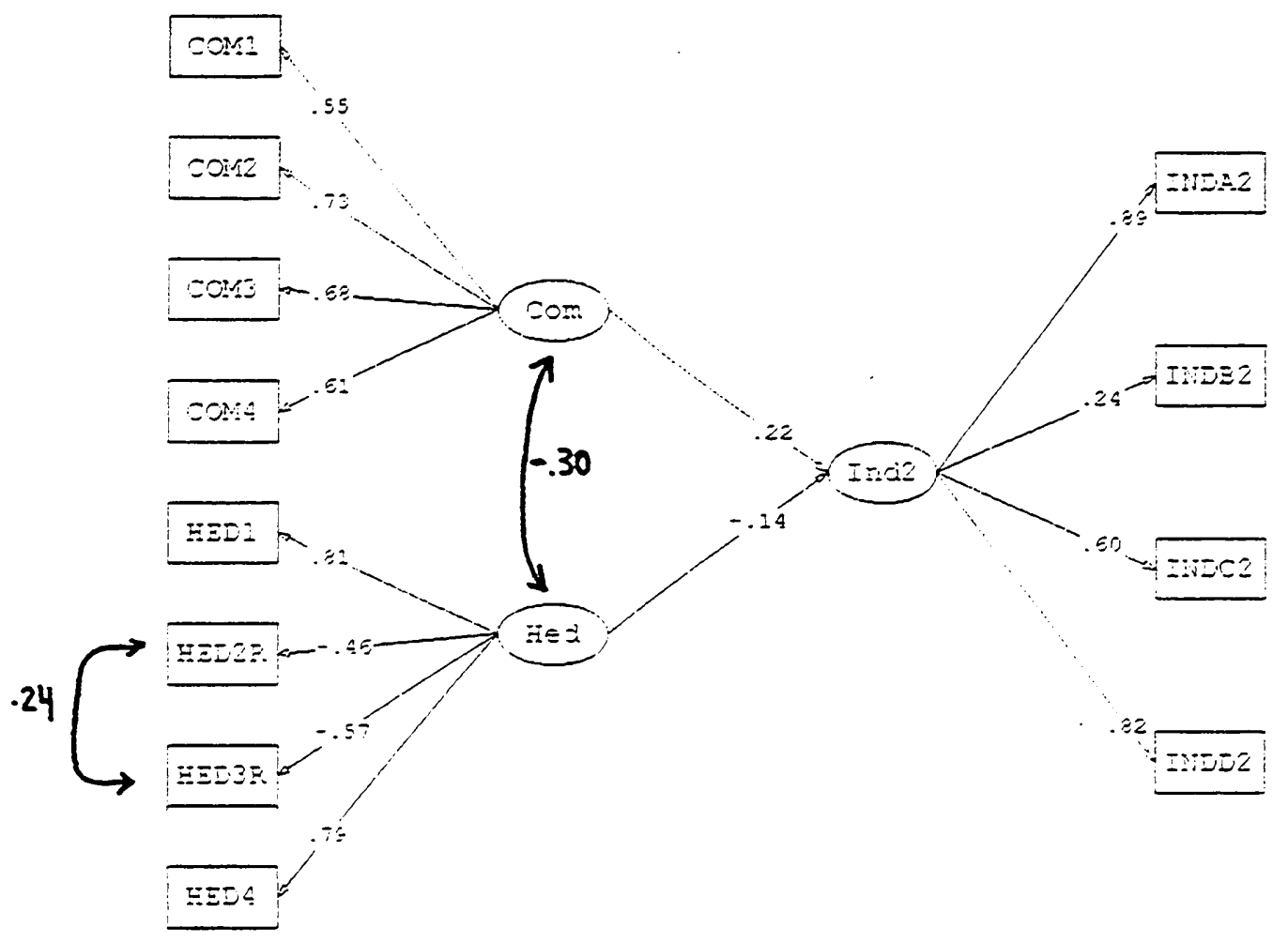

TANGY SPICES 
Table 27

Full Model Individualism - RO

Scenario 2 - Hispanics

t-values

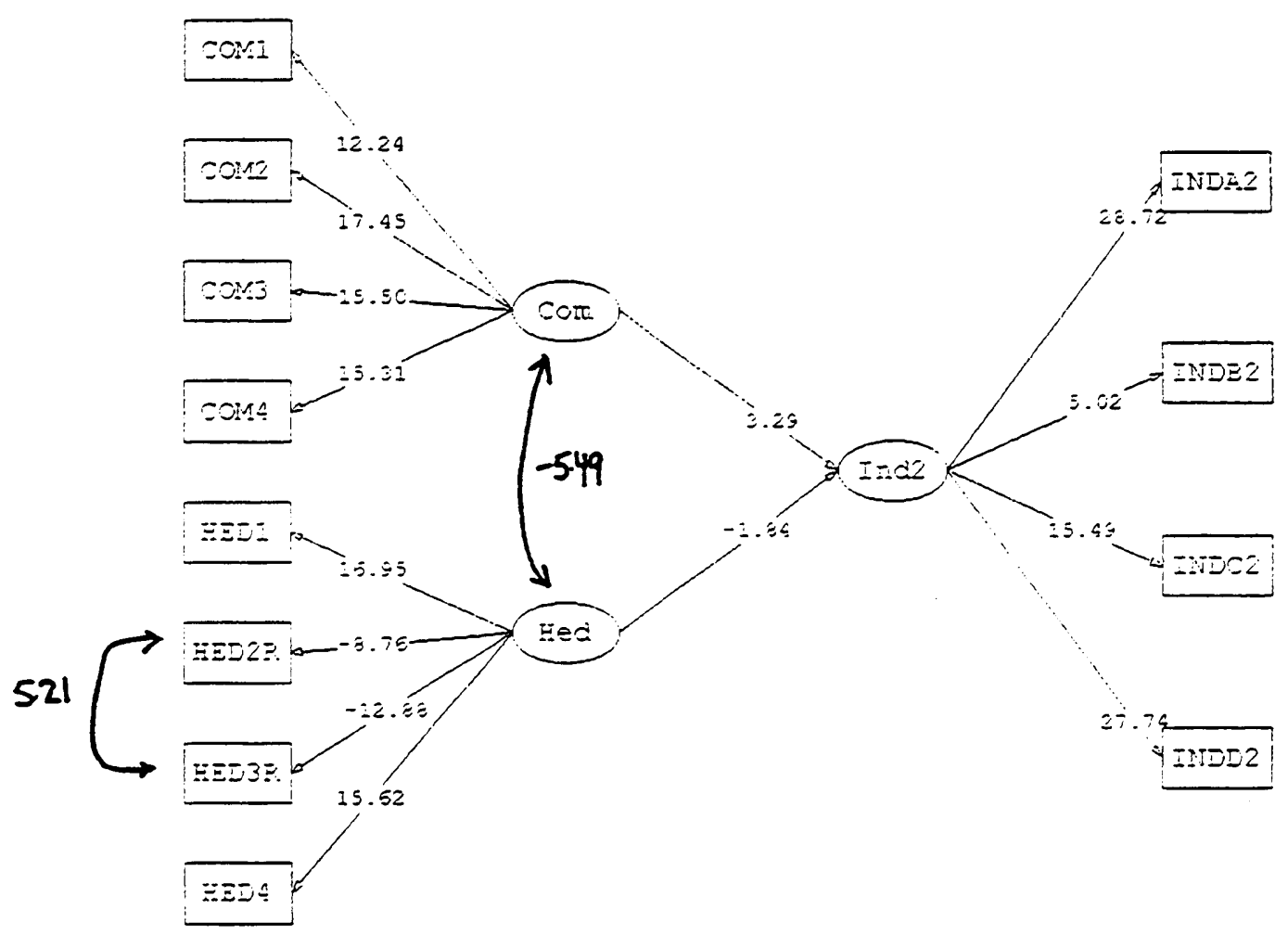

TANGY SPICES 


\section{Table 28}

Full Model Collectivism - MO

Scenario 2 - Anglos

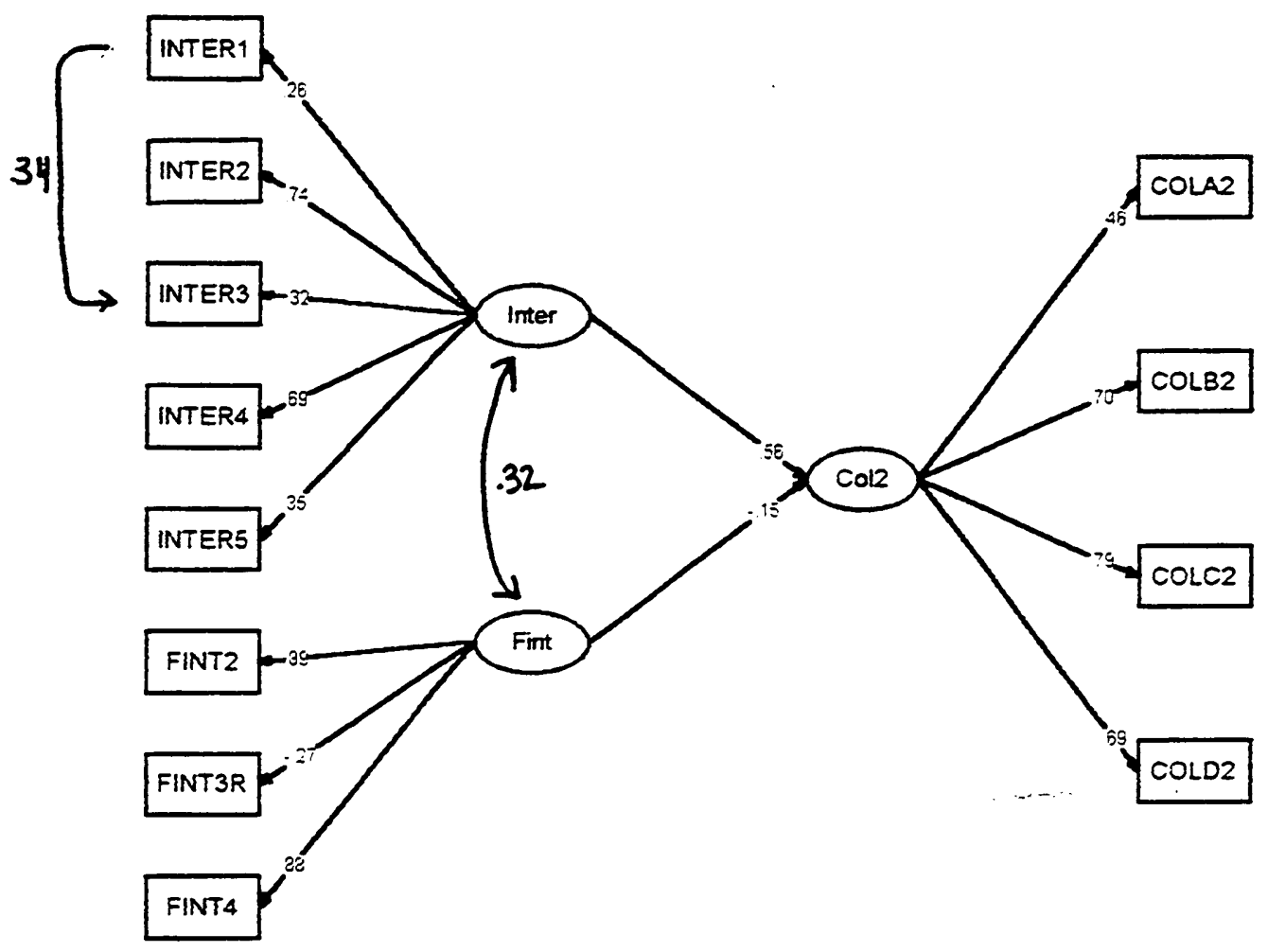

TANGY SPICES 
Full Model Collectivism - MO

Scenario 2 - Anglos

t-values

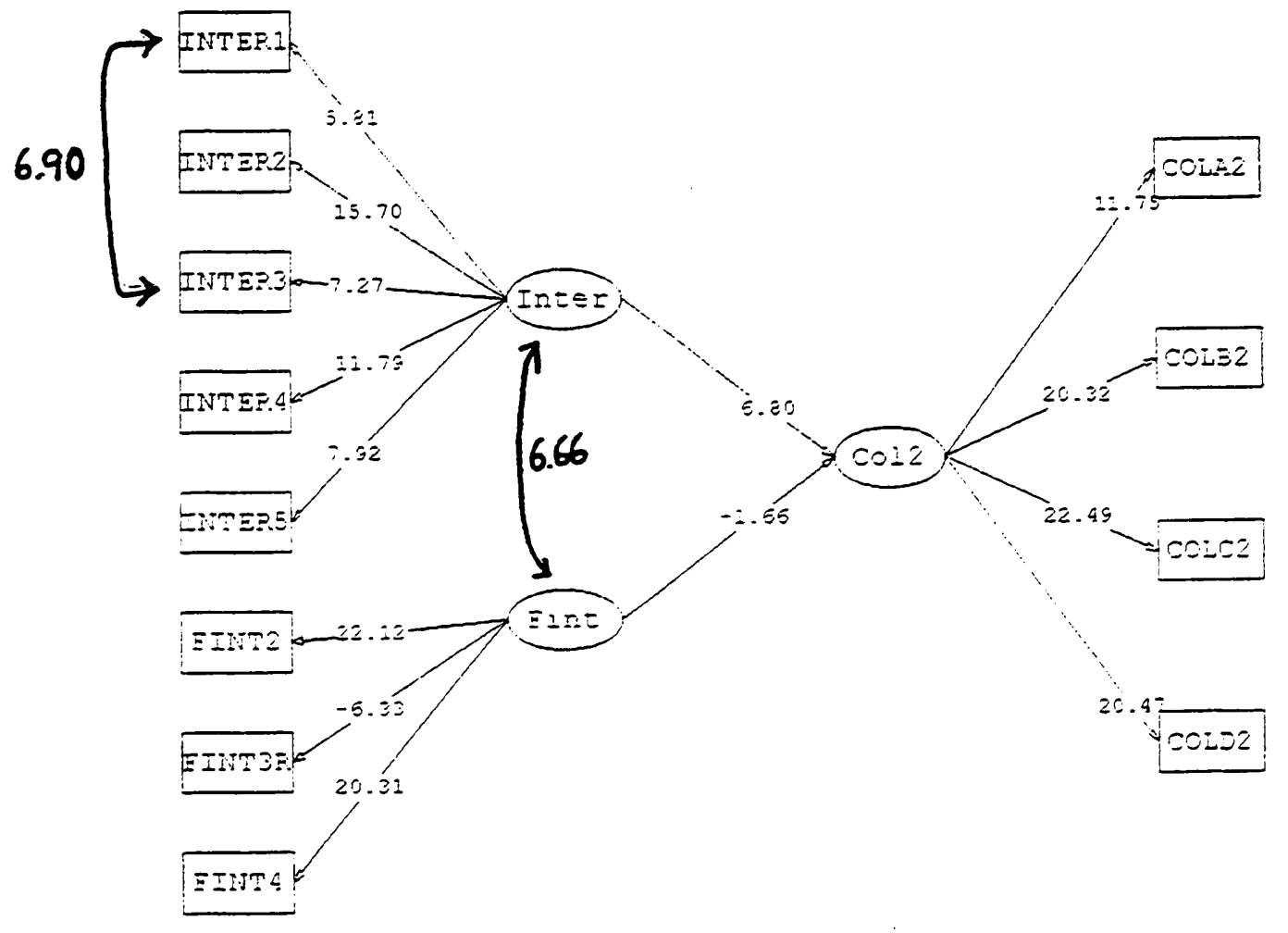

TANGY SPICES 
Table 29

Full Model Collectivism - MO

Scenario 2 - Hispanics

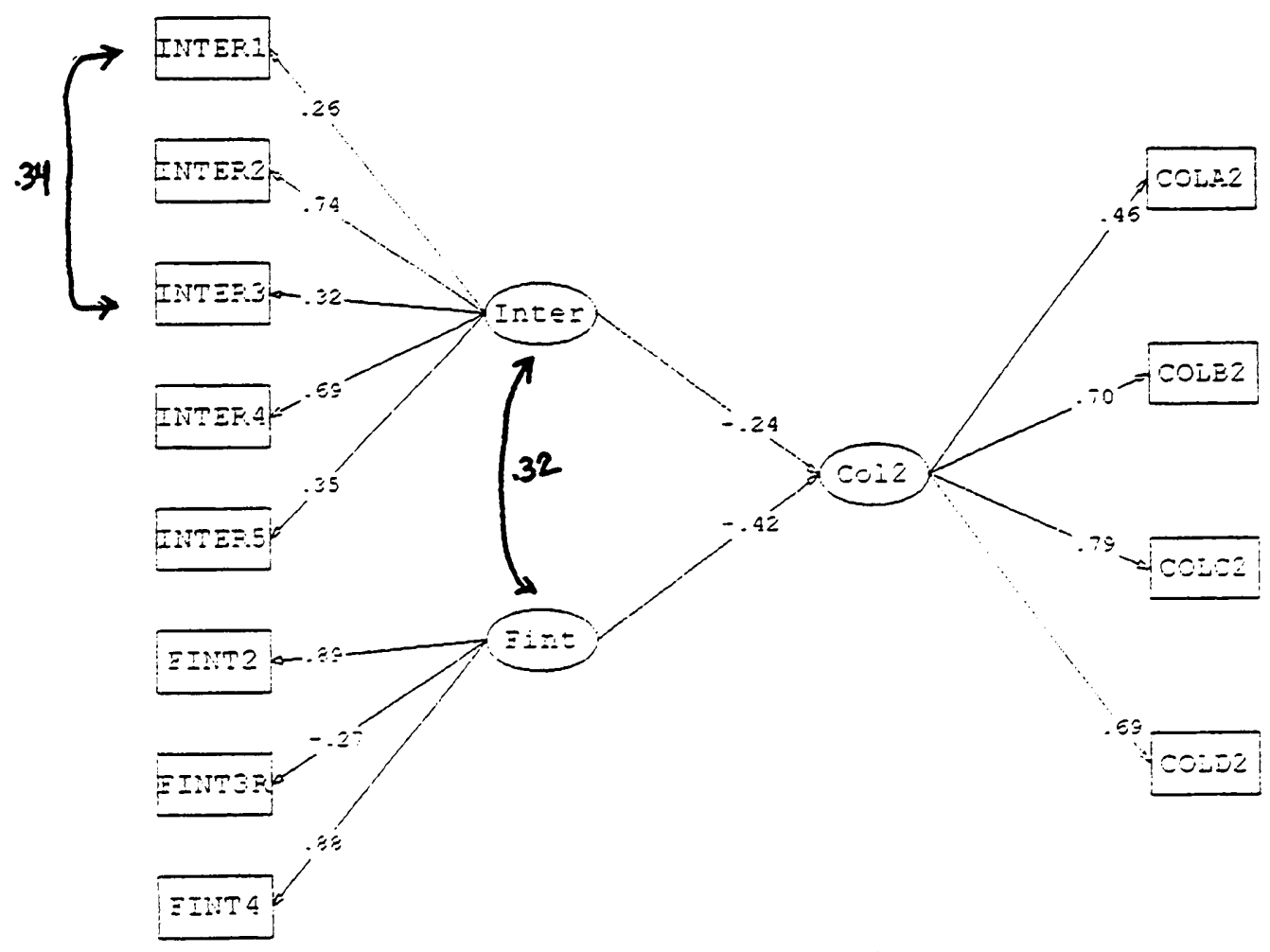

TANGY SPICES 
Full Model Collectivism - MO

Scenario 2 - Hispanics

$\mathrm{t}$-values

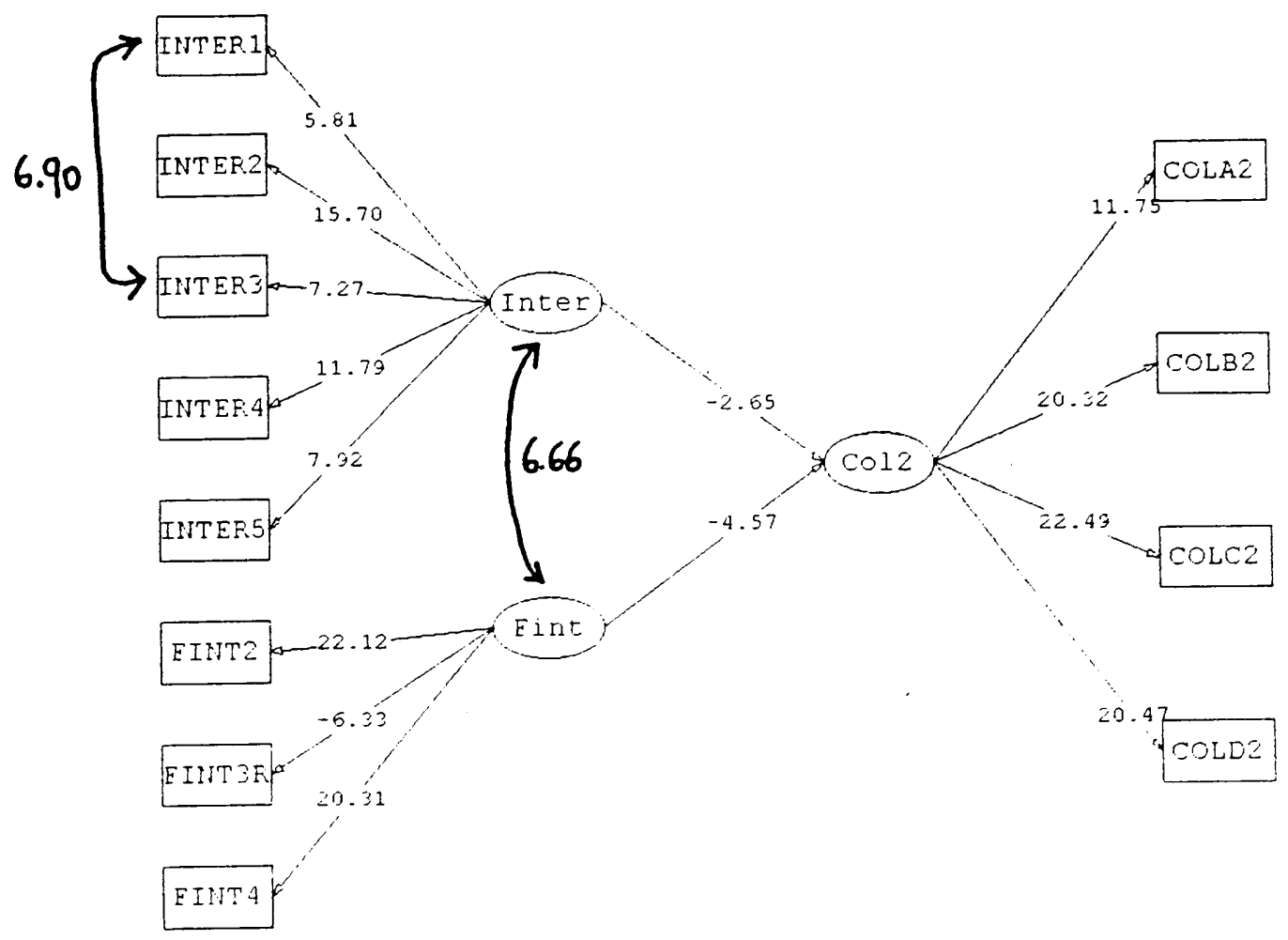

TANGY SPICES 
Table 30

Full Model Individualism - MO

Scenario 3 - Anglos

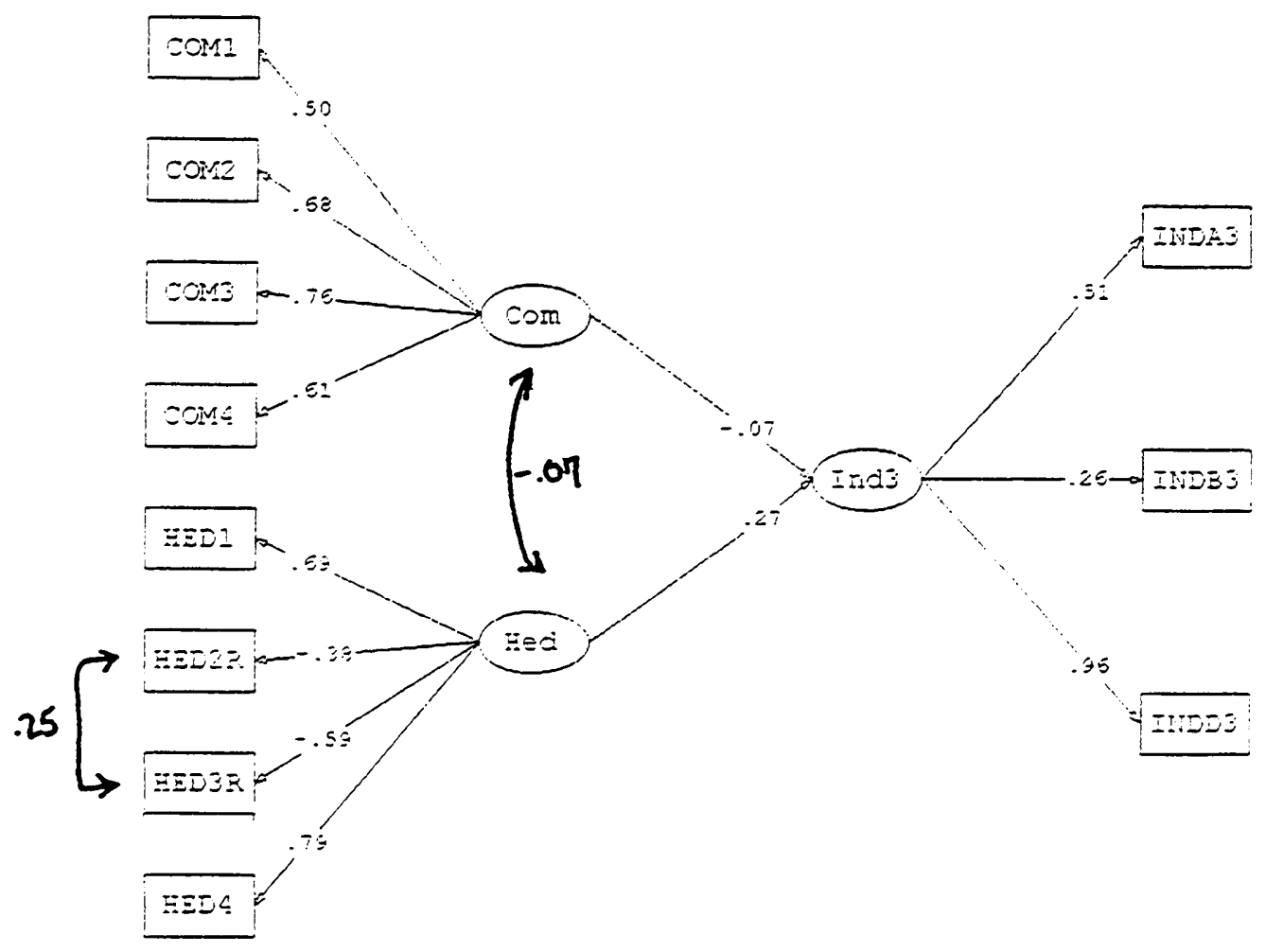

MICROCOMPUTER FIRM 
Table 30

Full Model Individualism - MO

Scenario 3 - Anglos

$t$-values

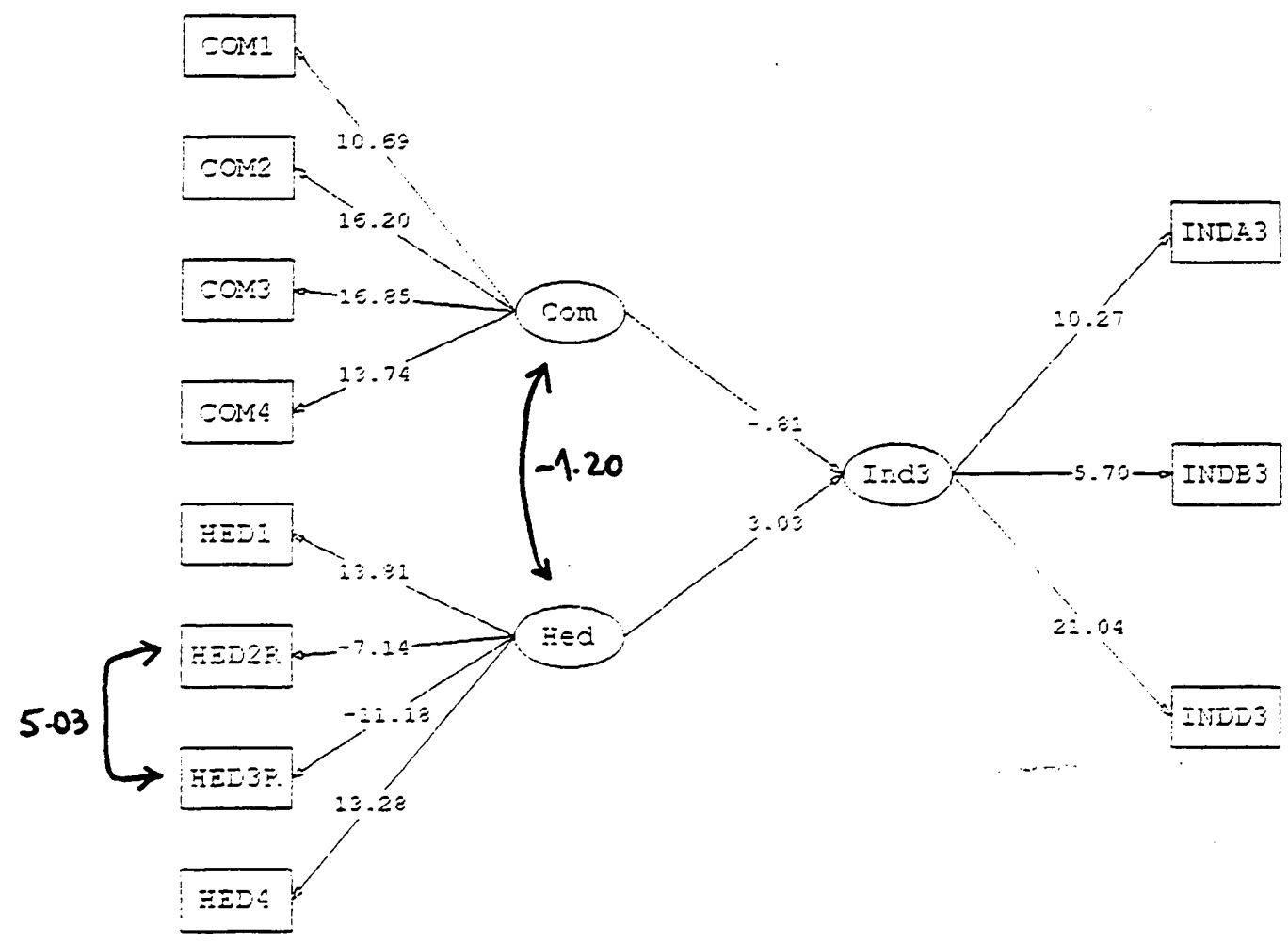

MICROCOMPUTER FIRM 
Table 31

Full Model Individualism - MO

Scenario 3 - Hispanics

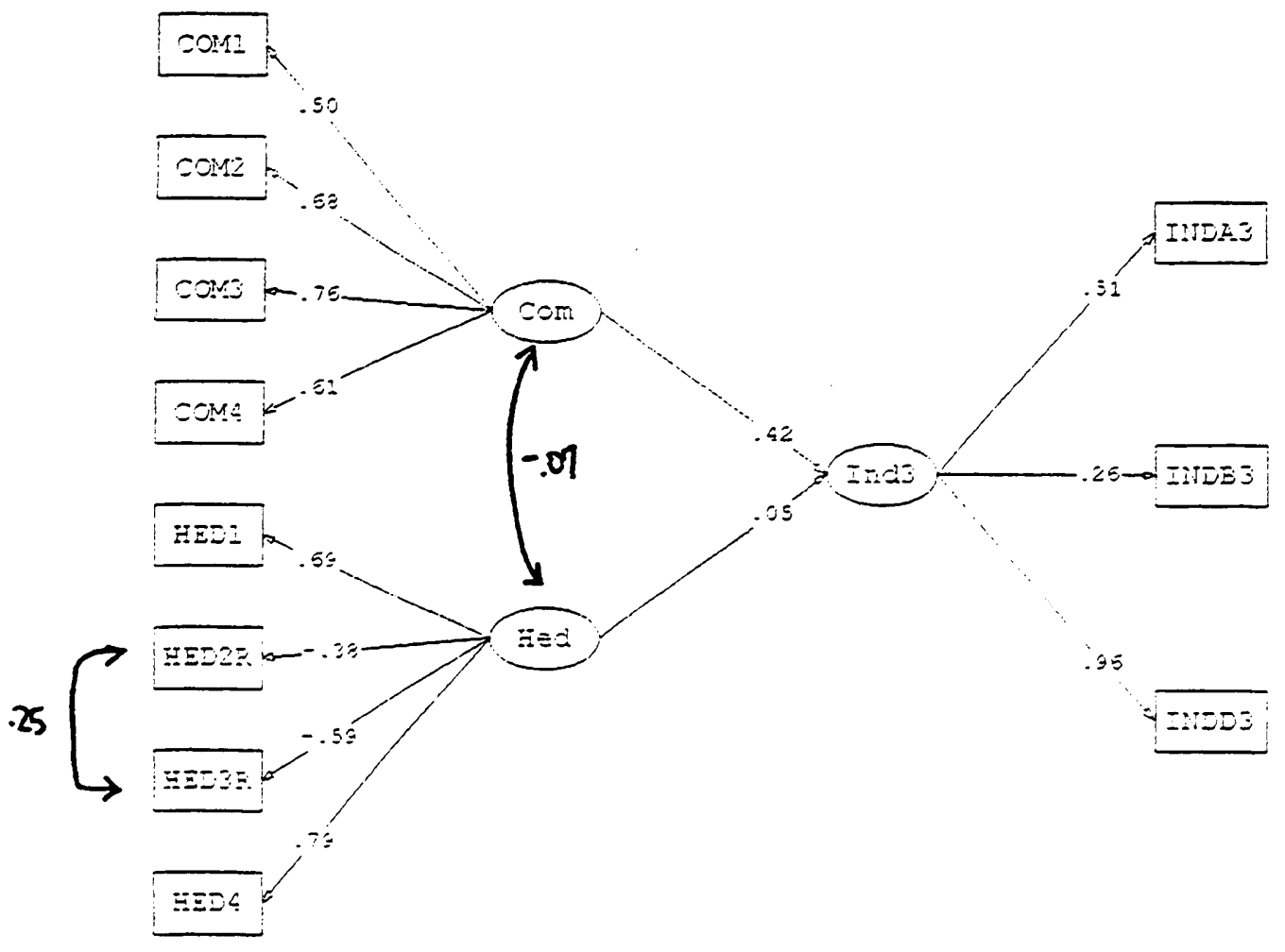

MICROCOMPUTER FIRM 
Table 31

Full Model Individualism - MO

Scenario 3 - Hispanics

t-values

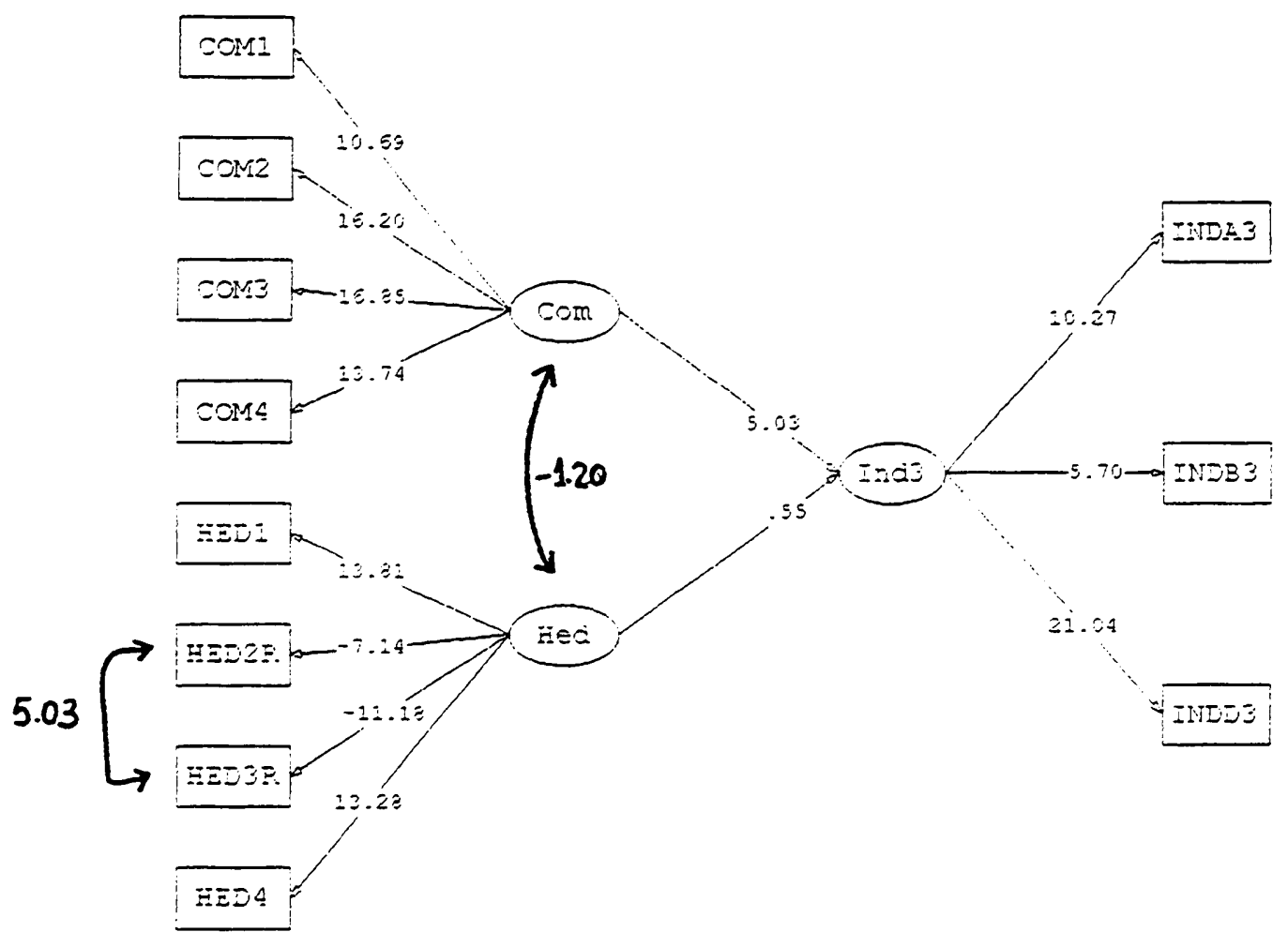

MICROCOMPUTER FIRM 
Full Model Collectivism - RO

Scenario 3 - Anglos

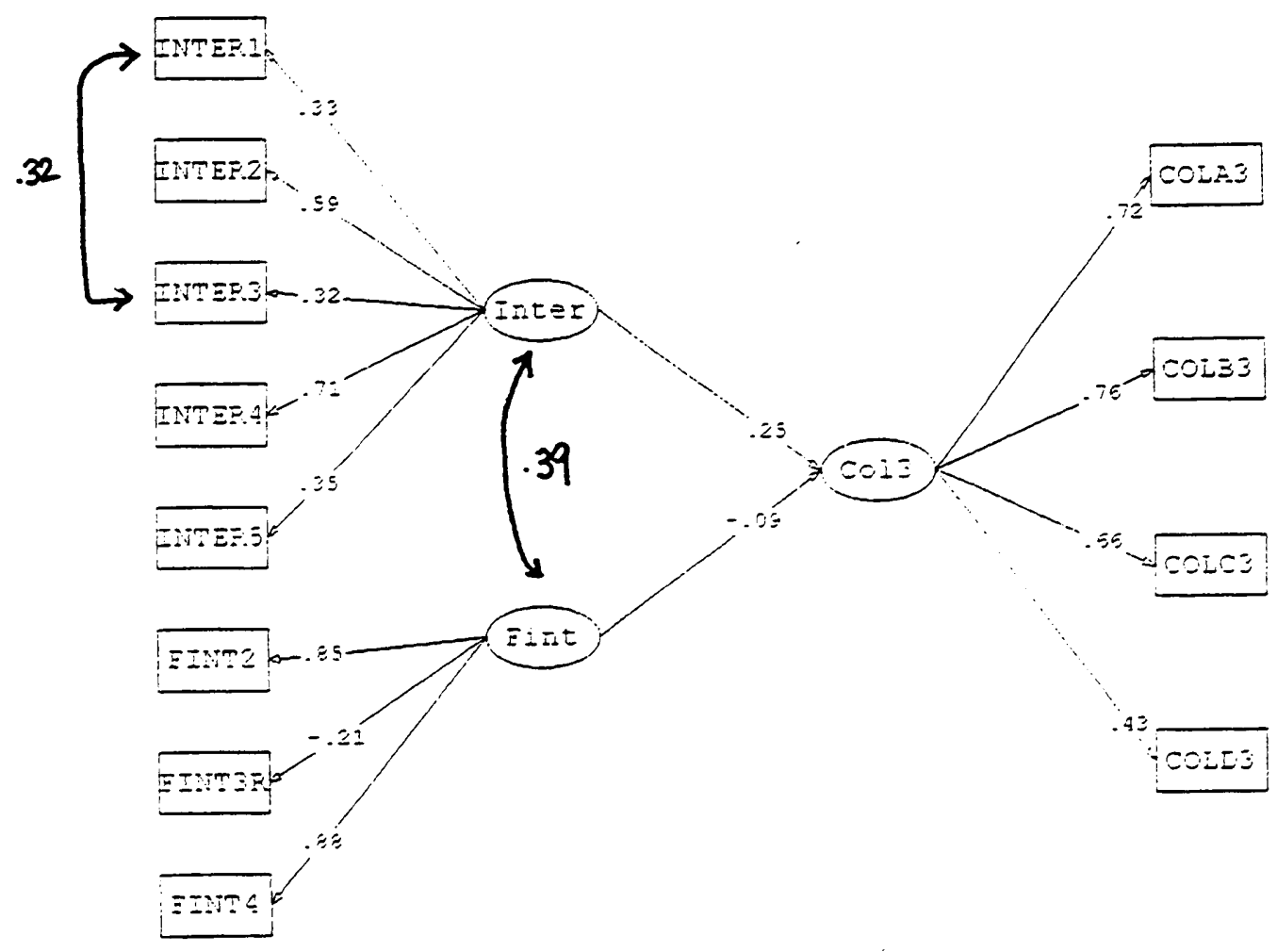

MICROCOMPUTER FIRM 
Table 32

Full Model Collectivism - RO

Scenario 3 - Anglos

t-values

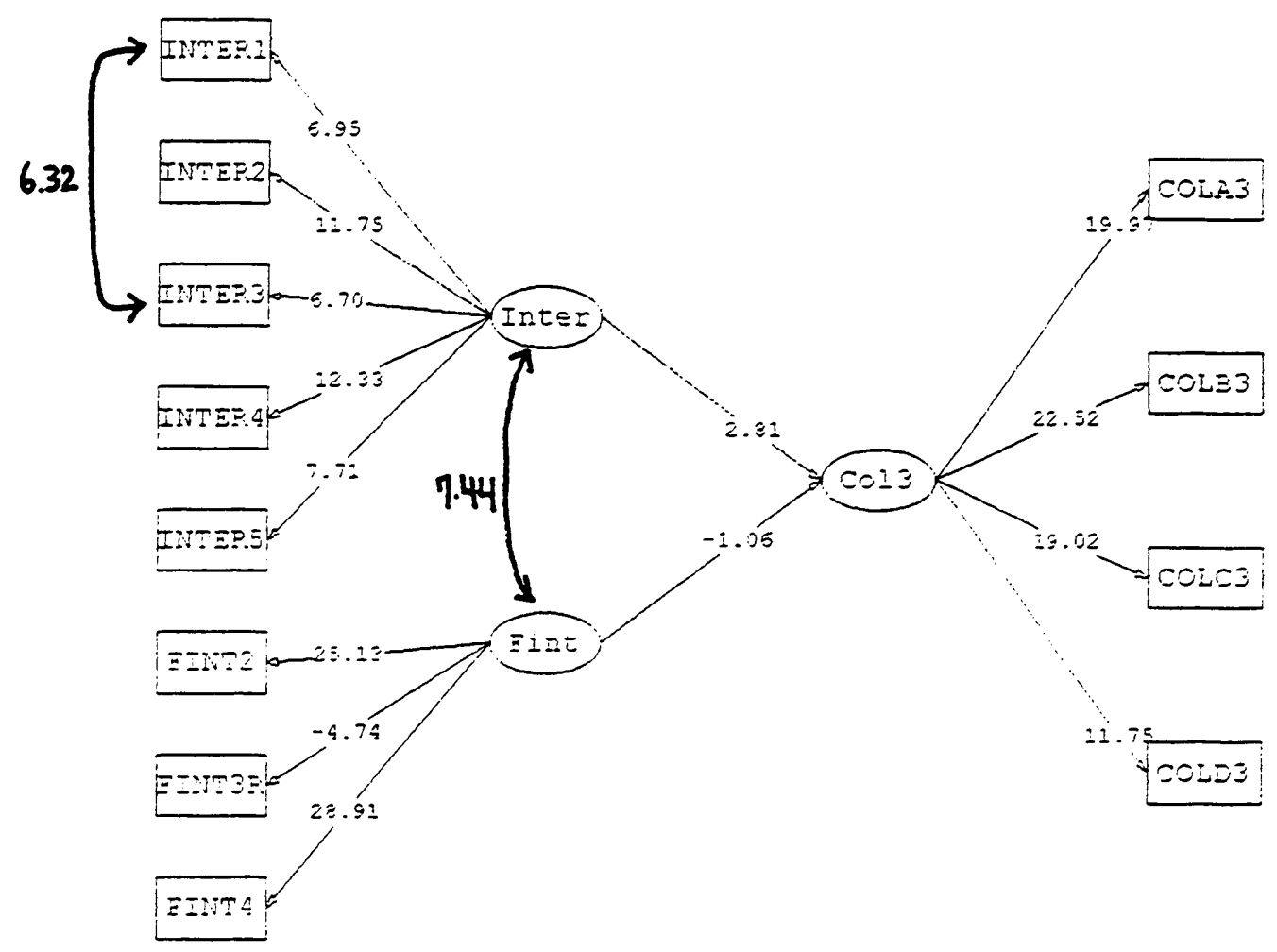

MICROCOMPUTER FIRM 
Table 33

Full Model Collectivism - RO

Scenario 3 -Hispanics

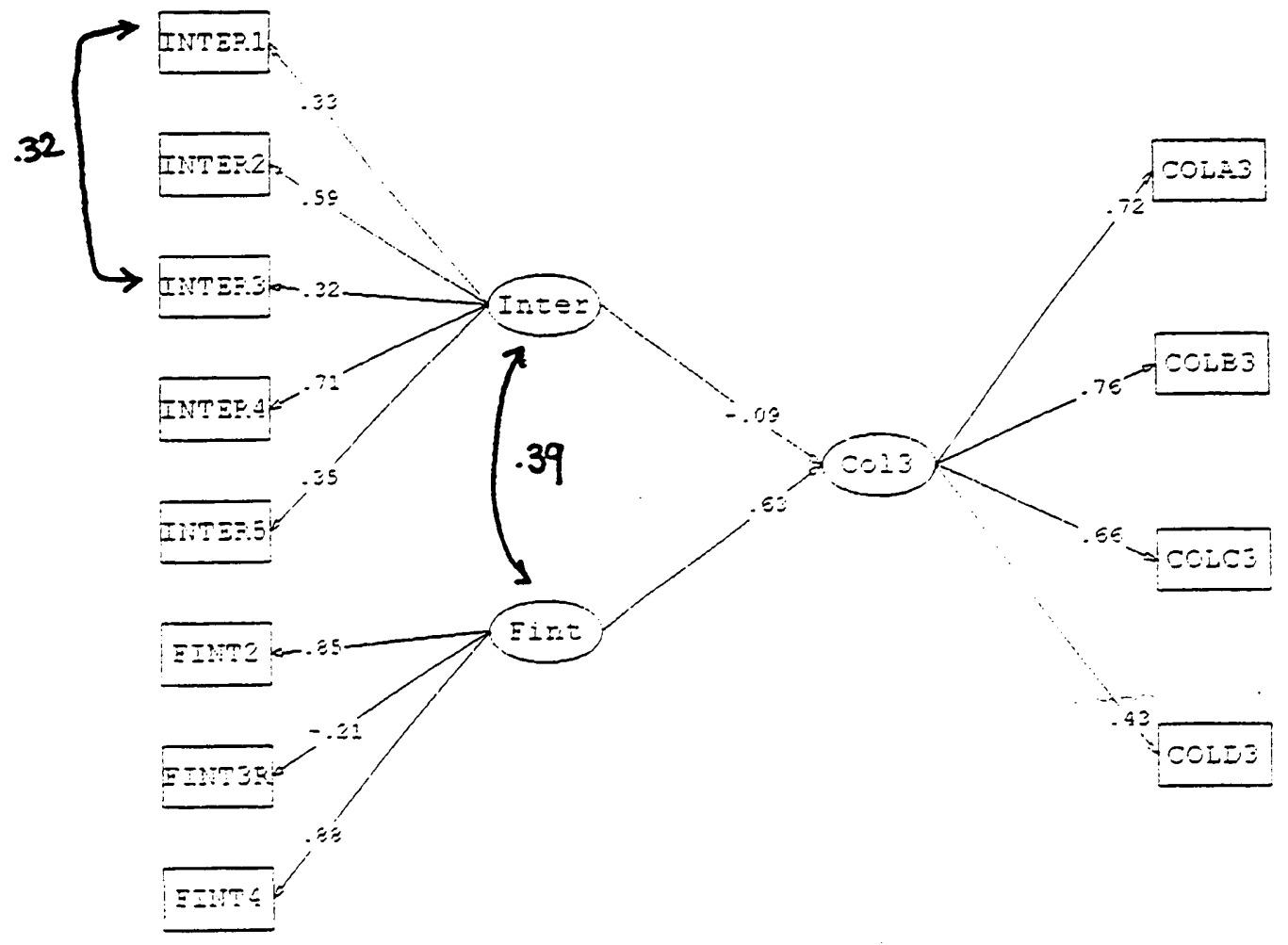

MICROCOMPUTER FIRM 
Table 33

Full Model Collectivism - RO

Scenario 3 -Hispanics

t-values

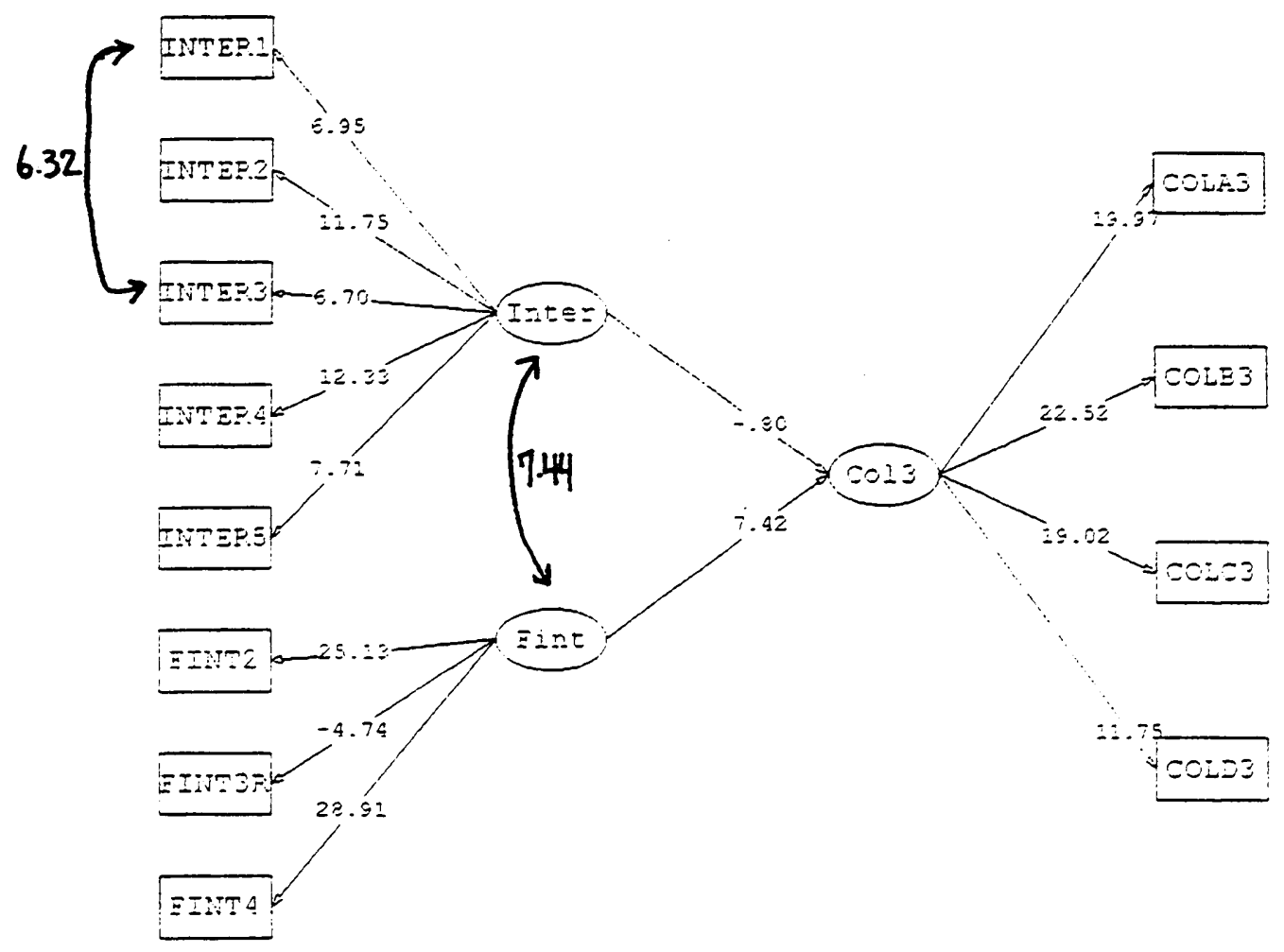


Table 34

Full Model Individualism - MO

Scenario 4 -Anglos

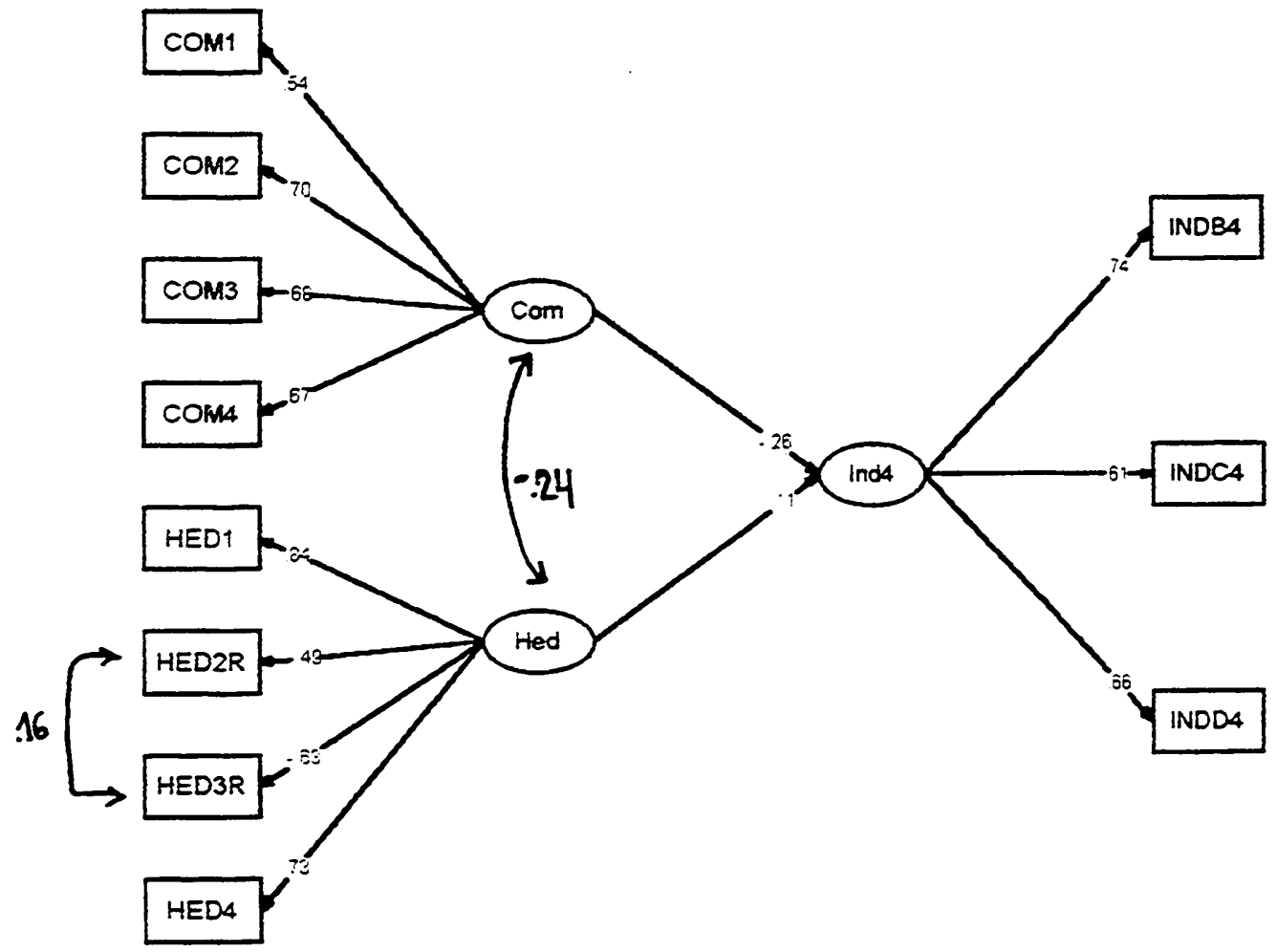

J\&P PUBLISHING CO. 
Table 34

\section{Full Model Individualism - MO \\ Scenario 4 -Anglos \\ t-values}

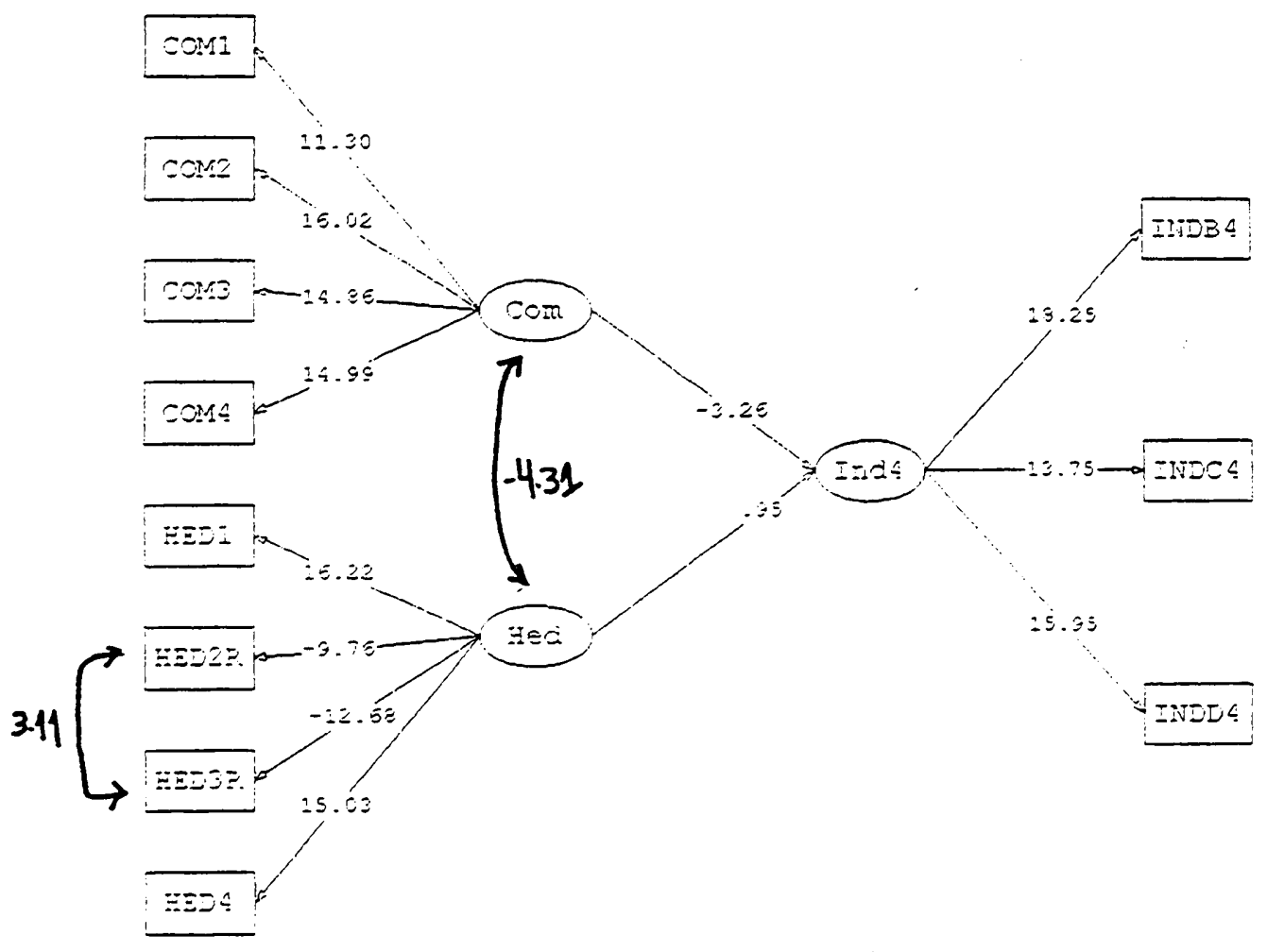

J\&P PUBLISHING CO. 
Table 35

Full Model Individualism - MO

Scenario 4 - Hispanics

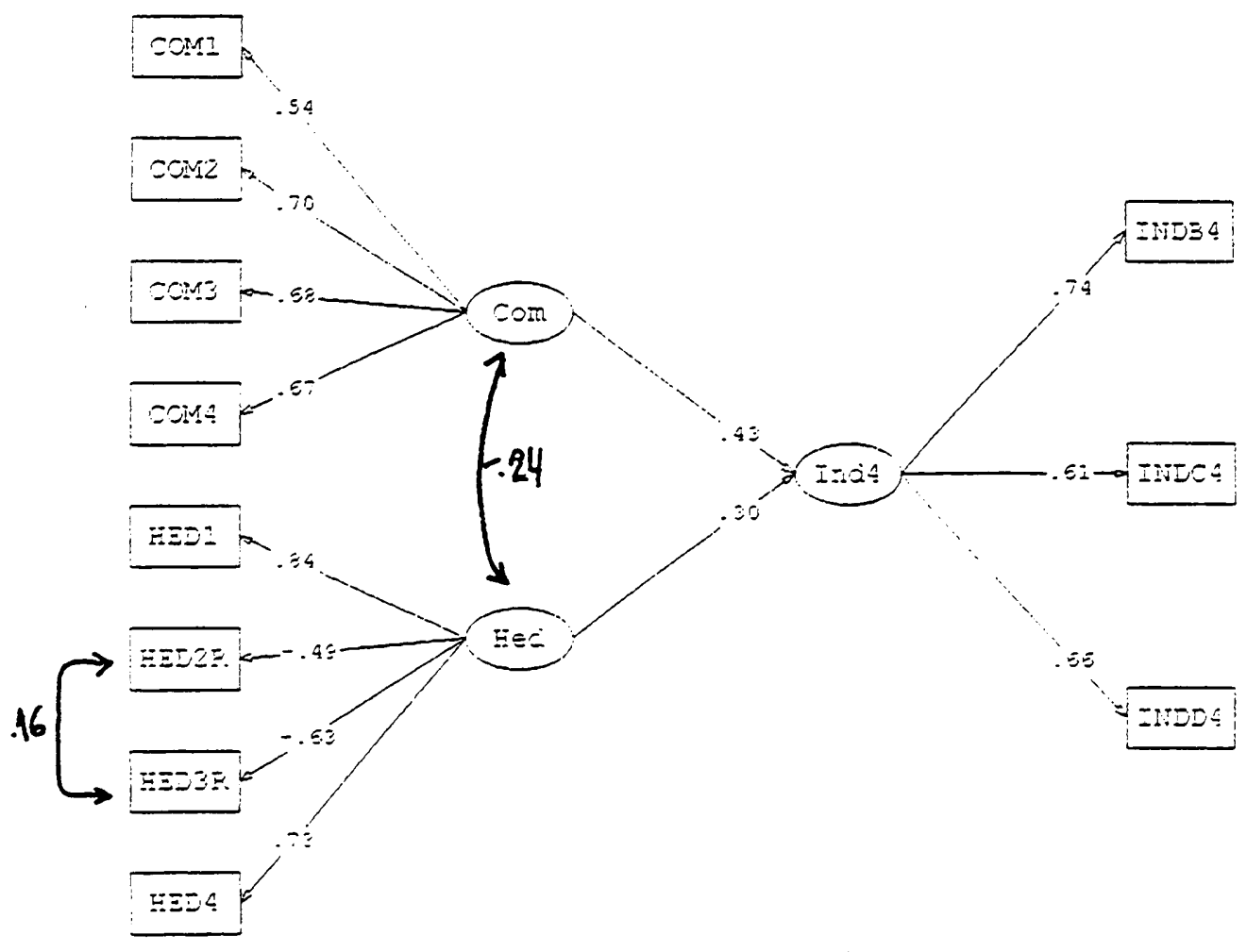

J\&P PUBLISHING CO. 


\section{Table 35}

\section{Full Model Individualism - MO \\ Scenario 4 - Hispanics \\ t-values}

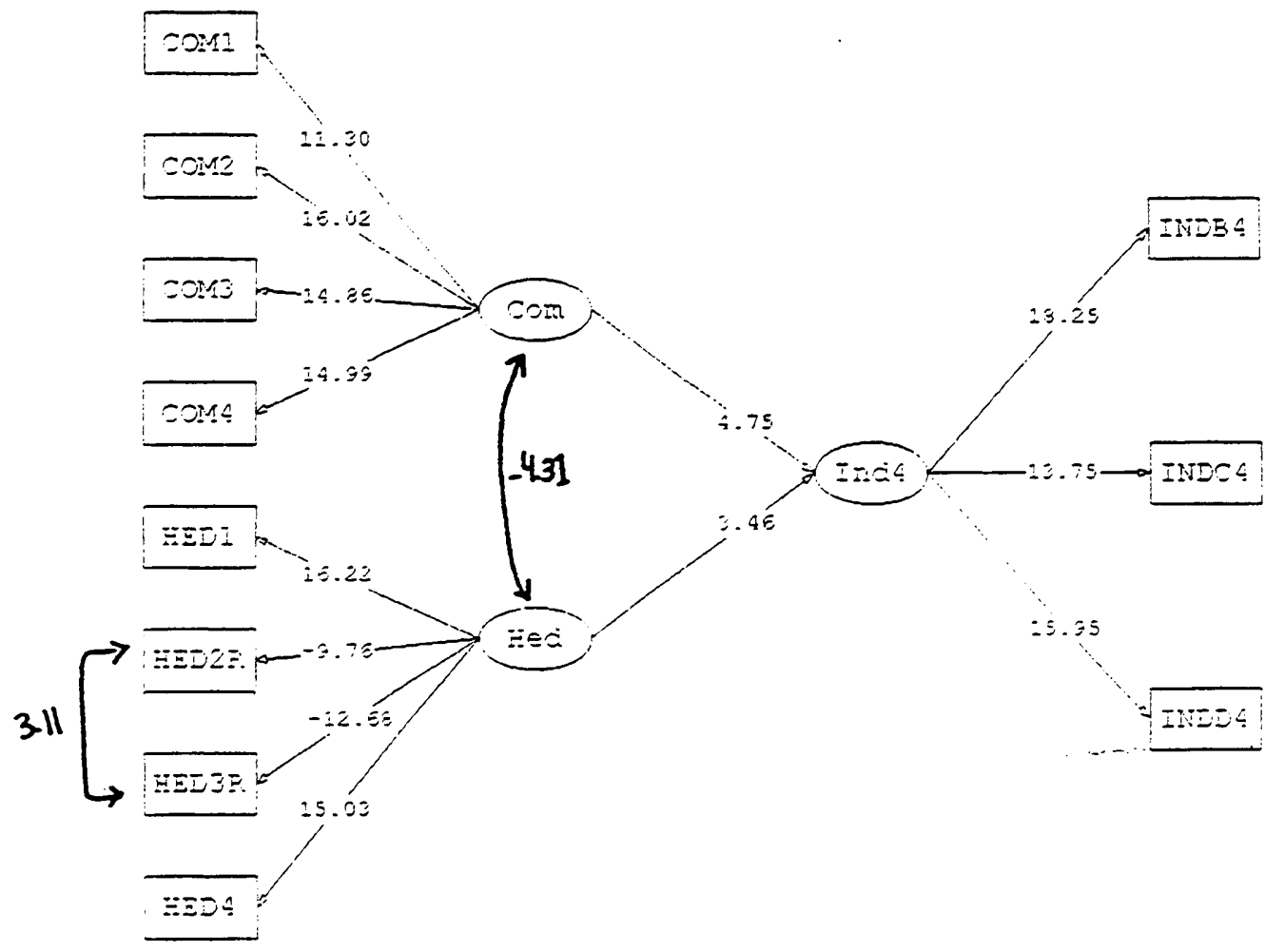

J\&P PUBLISHING CO. 


\section{Full Model Collectivism - RO}

Scenario 4 - Anglos

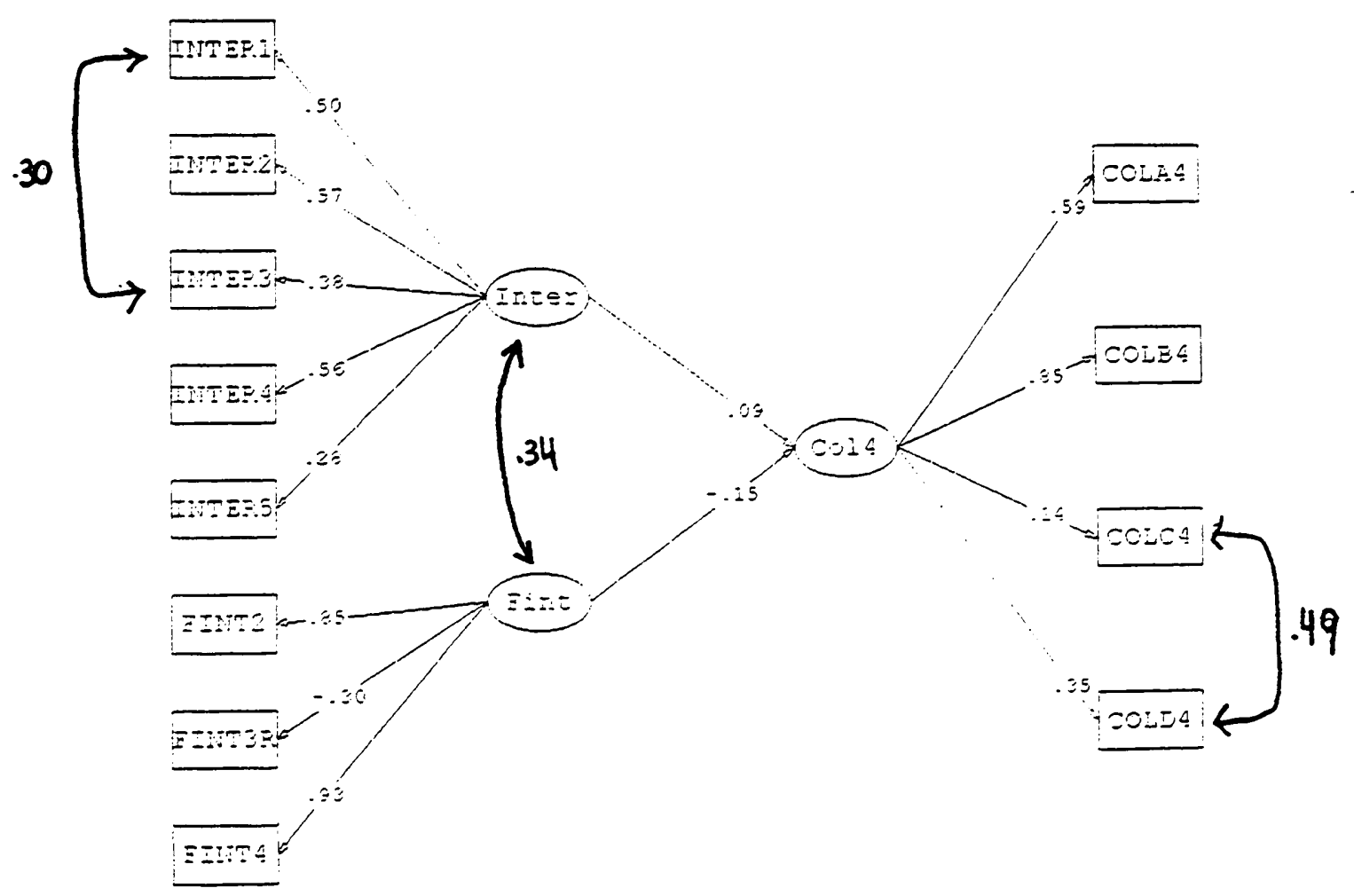

J\&P PUBLISHING CO. 
Full Model Collectivism - RO

Scenario 4 - Anglos

$t$-values

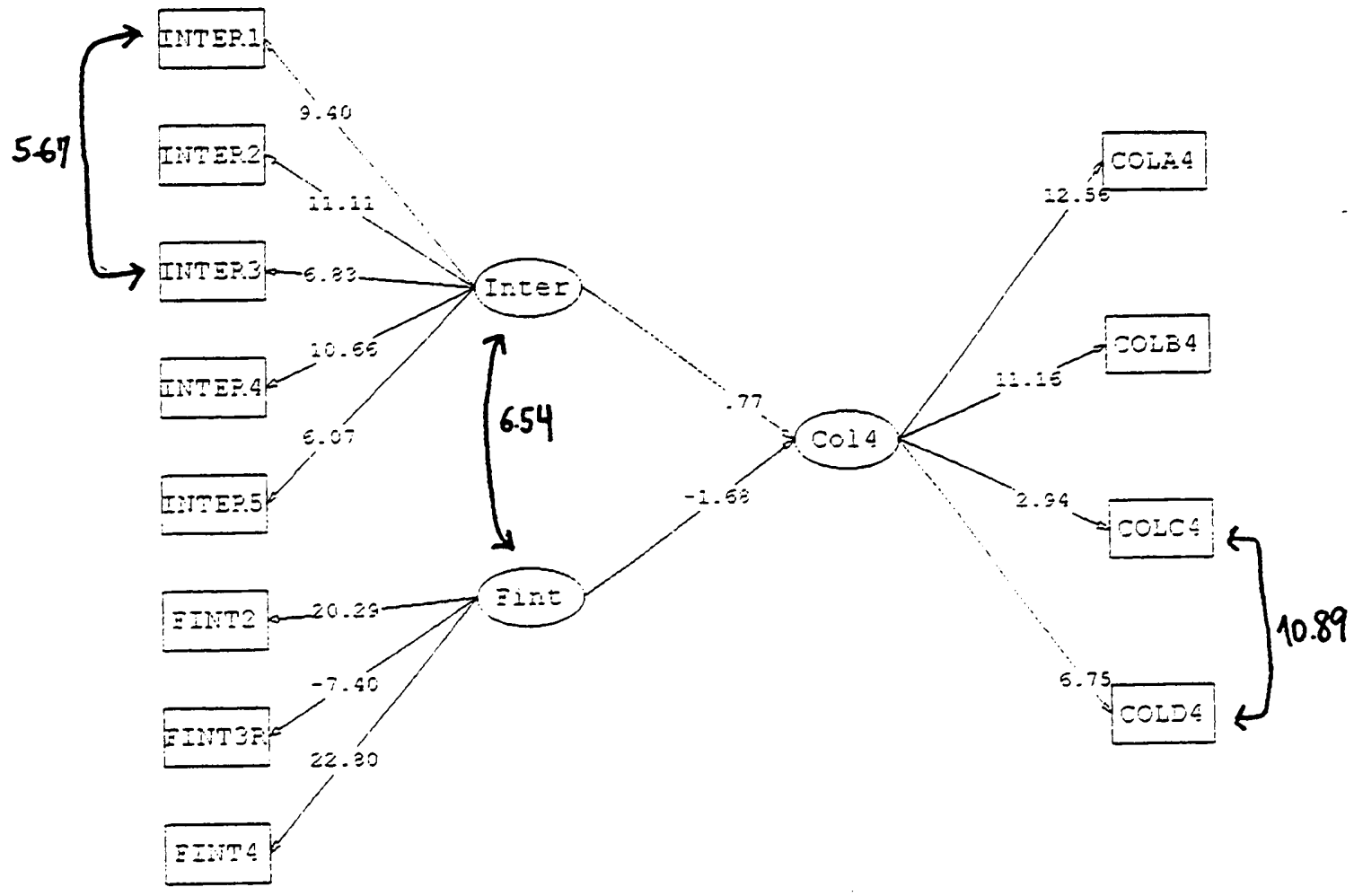

J\&P PUBLISHING CO. 
Table 37

Full Model Collectivism - RO

Scenario 4 - Hispanics

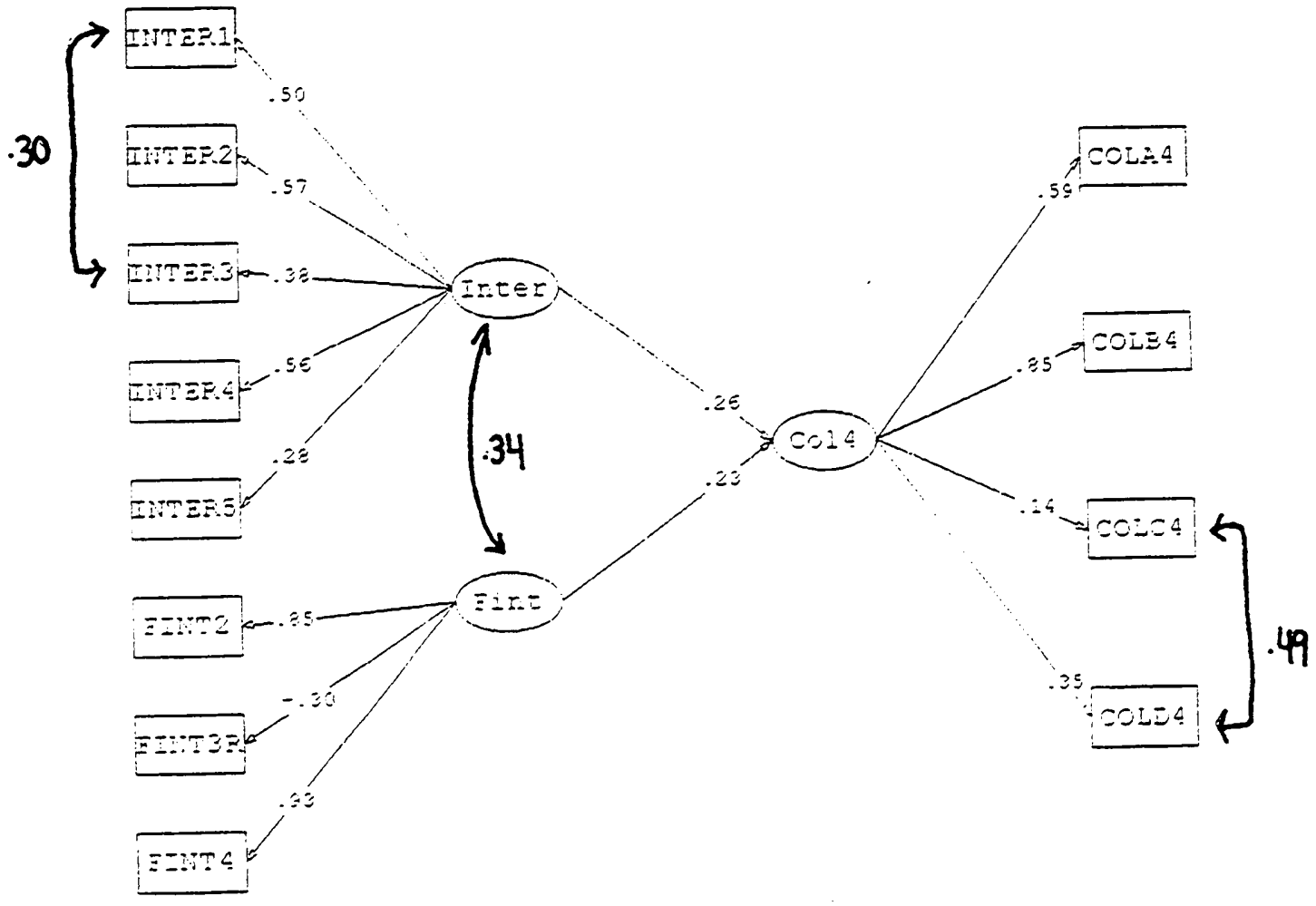

J\&P PUBLISHING CO. 


\section{Full Model Collectivism - RO}

Scenario 4 - Hispanics

$\mathrm{t}$-values

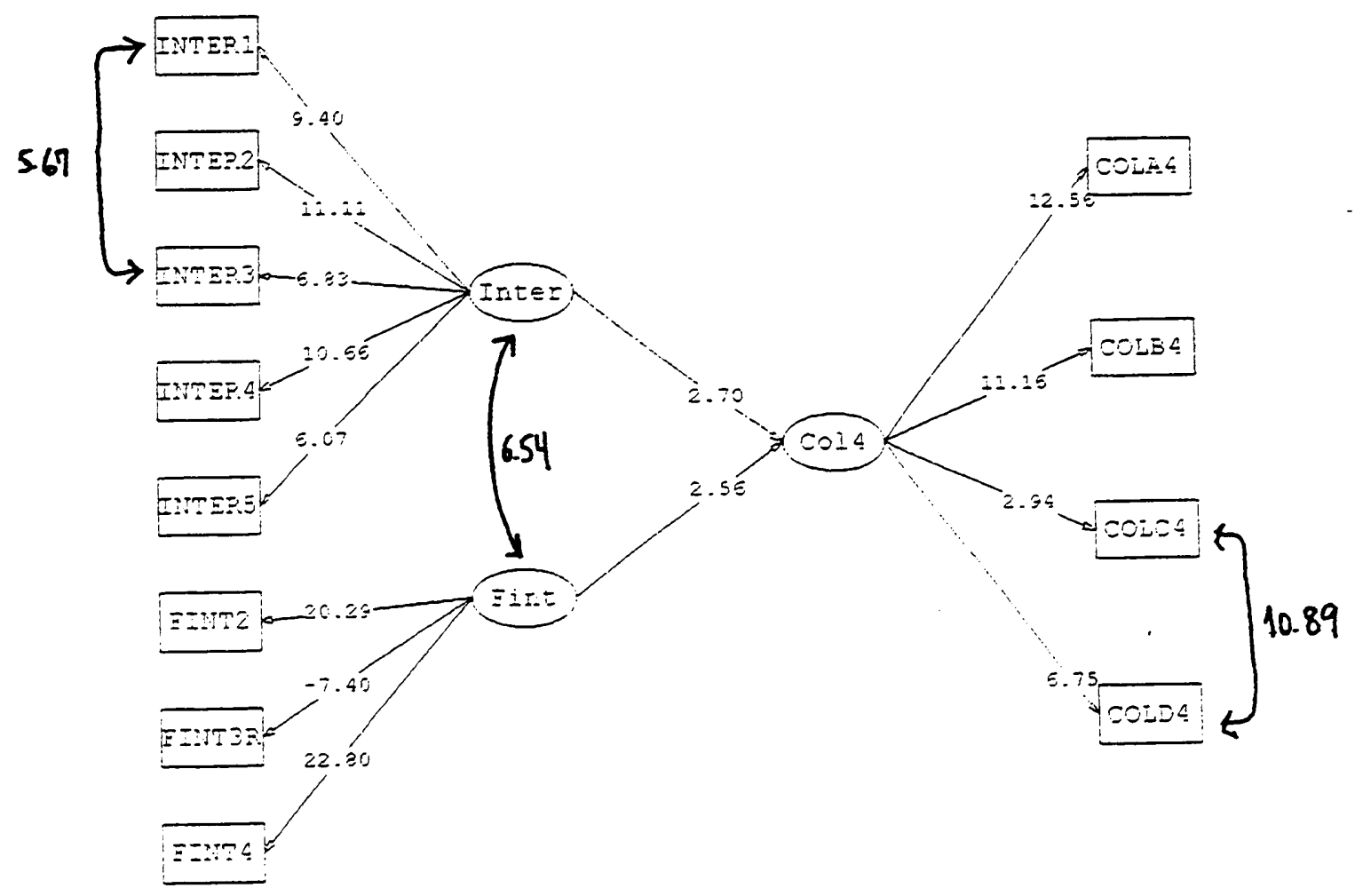

J\&P PUBLISHING CO. 
VITA

251 


\section{Ana Lucia Boamorte de Azevedo \\ Address: 820 84th Street \\ Miami Beach, F1 33141 \\ Telephone: (305)865-9423}

\section{Education:}

$\mathrm{PhD}$ in Business Administration at Florida International University - Concentration Area:

Organizational Behavior and Theory.

Masters in Business Administration at Coppead-Federal University of Rio de Janeiro (UFRJ), 1987-1988.

Bachelors in Economics at Fea-Federal University of Rio de Janeiro (UFRJ), 1982-1985 'Visconde de Cairu' Gold Medal.

\section{Dissertation Topic}

"The Role of Individualism and Collectivism as Predictors of Attributions for Unethical Behavior: An Empirical Examination Across Two Culturally Diverse Groups"

\section{Dissertation Committee}

Mary Ann Von Glinow (Chair), Daniel Robey, Karen Paul and Jerald Smith.

\section{Research Interests}

Organizational Cultures and Diversity, Cross-Cultural Influences on Human Resources Management and on Organizational Theorizing, Business Ethics and Social Responsibility Issues.

\section{Teaching Interests}

Organizational Behavior, Organizational Theory, Comparative Management, Business Ethics, Business \& Society. 


\section{Work Experience}

Teaching Assistant at Florida International University, Fall 1995 - Course: Business and Society.

Member of the FIU Research Team in charge of the Project "Local Challenges in the Global Economy", developed in collaboration with the Harvard Business School. Over the course of 1994, this group of professors and doctoral students administered questionnaires, conducted interviews with business executives and delivered study results in a conference to the South Florida business comunity in March, 1995.

Teaching Assistant at Florida International University, 1992-1993 - Courses: Management \& Entrepreneurship; Teaching Evaluations ranging from 3.20 to 3.50. Helped students in developing several business plans for start-up ventures.

Research Assistant at Florida International University, 1989-1991

- Projects: Motivation needs, Organizational Culture.

Management Consultant at Mito International Corporation, 1992. Helped implement an integrated accounting software that automated all the main activities of this import/export company.

Economist at Natron Engineering \& Projects, Research Dept., Rio de Janeiro/Brazil, 1986-1987. Participated in feasibility studies for engineering projects, specially with respect to market and pricing analysis and overall economic evaluation.

\section{Publications}

Richard Hodgetts and Ana Azevedo, "Total Quality Management: Licoes para a America Latina", Revista de Administracao Publica, RJ/Brazil, vol.28, no.4, 1994.

Daniel Robey and Ana Azevedo, "Cultural Analysis of the Organizational Consequences of Information Technology", Accounting, Management and Information Technologies, vol.4, no.1, 1994.

Len Chusmir and Ana Azevedo, "Motivation Needs of Sampled Fortune-500 CEOs: Relations to Organization Outcomes", Perceptual \& Motor Skills, vol.75, 1992. 


\section{Conference Proceedings}

Daniel Robey and Ana Azevedo, "Information Technology and Organizational Culture", Proceedings of the Annual Conference of the Administrative Sciences Association of Canada, Quebec, June 1992.

Len Chusmir and Ana Azevedo, "Impacts of Needs for Achievement and Power on Corporate Sales and Profits", Proceedings at the Academy of Management Southwest division, March 1991.

\section{Conference Participation}

Deborah Vidaver Cohen, Lori Zalka, Shawnta Friday, Ana Azevedo and Karen Paul, "Global Competitiveness and the Hidden Costs of Crime: A Study of BusinessStakeholder Relations in the Miami Metropolitan Area". Paper presented at the Annual Conference of the International Association of Business and Society, Austria, June 1995.

Ana Azevedo, Karen Paul and Mary Ann Von Glinow, "An Integrative Framework for Cross-Cultural Research on Business Ethics", Annual International Conference of the Society for the Advancement of Socio-Economics, France, July 1994.

Mahatapa Palit and Ana Azevedo, "Summary of Competitive Papers-1994 BALAS Proceedings", Annual Conference of the Business Association of Latin American Studies, Miami, April 1994.

\section{Honors}

Doctoral Schoolarship from the Brazilian Government, 1990-1994.

Participated in SIM Consortium at the Academy of Management Meetings, Dallas, August 1994.

\section{Professional Affiliation}

Member of the Academy Of Management Association. 